\title{
Non reciprocal passive components on LTCC ferrite substrate
}

\author{
by \\ Shicheng Yang \\ A thesis submitted to \\ Carleton University's Faculty of Graduate and Postdoctoral Affairs \\ in partial fulfillment of the requirements for the degree of
}

\section{Doctor of Philosophy in Electrical Engineering}

Ottawa-Carleton Institute for Electrical and Computer Engineering Carleton University Canada

October, 2015

(C) Copyright

Shicheng Yang, 2015 
The undersigned hereby recommends to

Carleton University's Faculty of Graduate and Postdoctoral Affairs

acceptance of the thesis

\title{
Non reciprocal passive components on LTCC ferrite substrate
}

\author{
submitted by Shicheng Yang
}

in partial fulfillment of the requirements for the degree of

Doctor of Philosophy in Electrical Engineering 
Professor Odile Picon, Université Paris-Est Marne-la-Vallée, France, Referee

Professor Arne Jacob,

University Hamburg-Harburg, Germany, Referee

Professor, Langis Roy,

Carleton University, Canada, Thesis Supervisor

Professor, Joey R. Bray, Royal Military College, Canada, Examiner

Adjunct Professor, Rony Amaya, Carleton University, Canada, Examiner

Professor, Philippe Ferrari, Université Joseph Fourier, France, Examiner

Maître de conférence HDR, Bernard Bayard, Université Jean Monnet de Saint Etienne, France, Examiner

Professor, Didier Vincent, Université Jean Monnet de Saint Etienne, France, Thesis Supervisor

Professor, Barry Syrett, Carleton University, Canada, Thesis co-Supervisor 
Ottawa-Carleton Institute for Electrical and Computer Engineering Carleton University Canada

October, 2015 


\section{Abstract}

This thesis investigates passive non-reciprocal components (mainly circulators) based on ferrite Low Temperature Co-fired Ceramic (LTCC) substrate. The external magnets used in conventional circulators must be strong to overcome the ferrite's demagnetization field. The novel circulator presented herein uses an embedded winding within the ferrite substrate to magnetize the material from the inside, thereby significantly reducing the demagnetization effects. Because of the controllability of the bias field, the resulting device is also multifunctional: when the windings are energized by a current, the device operates as a dynamic circulator in which the circulation direction can be changed by switching the direction of the current. Unlike other LTCC circulators with external magnets, the proposed device can even operate as a power splitter by removing the bias current. A circulator prototype has been characterized in three states: unbiased, biased by winding and biased by winding and external magnets. When no current is applied, the transmission of each port is about $-5 \mathrm{~dB}$ with a return loss better than $20 \mathrm{~dB}$ at $14.8 \mathrm{GHz}$. When a current of $300 \mathrm{~mA}$ is injected into the winding, the measured insertion loss and isolation of the circulator is approximately $3 \mathrm{~dB}$ and $8 \mathrm{~dB}$, respectively, whereas the return loss is better than $20 \mathrm{~dB}$ at $14.2 \mathrm{GHz}$. When external magnets are added in addition to the current of $200 \mathrm{~mA}$, the insertion loss and isolation is improved to $1.6 \mathrm{~dB}$ and $23 \mathrm{~dB}$, respectively at $14.2 \mathrm{GHz}$. The variation of the circulator's working frequency is $0.6 \mathrm{GHz}$. This is 
achieved firstly by the change of the internal magnetization $\mathrm{M}$ when the dc current is less than $120 \mathrm{~mA}$, then the heat in the substrate due to the winding introduces more frequency shifting. The total size $\left(\mathrm{L}^{*} \mathrm{~W}^{*} \mathrm{H}\right)$ is $8 \mathrm{~mm}^{*} 8 \mathrm{~mm}^{*} 1.1 \mathrm{~mm}$.

The conventional Y-junction stripline circulator has one ferrite disk surrounded by a dielectric material. The design of the circulator presented here uses only the ferrite LTCC material. The material exposed under the magnetic bias shows the ferrite properties and the remaining shows the dielectric properties. It has been demonstrated that a passive ferrite component like circulator could be realized on the ferrite LTCC substrate without adding other materials. Therefore, this circulator could be easily integrated with other rf components such as antennas, amplifiers, filters, etc in System-on-Package (SoP).

The conventional circulator with stripline structure is designed to operate at a specific frequency. To expand the bandwidth, we usually work on the shape of the circulator's center conductor, or operate the circulator in the "edge-guided" mode. In order to increase the bandwidth, the 3D design ability of LTCC technology can be used. In this thesis, we present a new wideband circulator operating at K-band. The design is realized by stacking two circulators (gyrators) with different working frequencies into the ferrite LTCC substrate to expand the overall bandwidth. The obtained bandwidth by the simulator Ansys HFSS is $11 \mathrm{GHz}(15.5 \mathrm{GHz}$ to $26.5 \mathrm{GHz})$ which covers the K-band. The reflection and isolation are over $20 \mathrm{~dB}$ and the insertion loss is between $1.5 \mathrm{~dB}$ and $3 \mathrm{~dB}$.

In this thesis, we investigate the original circulator design fully embedded in ferrite LTCC, study the performance of a ferrite LTCC circulator with integrated winding and present coplanar circulators and a wide-band circulator based on the ferrite LTCC substrate. All designs are realized in Ansys HFSS, Ansys Maxwell 3D and CST Studio at University of Saint Etienne, Laboratory Hubert Curien UMR CNRS 5516 and at 
Carleton University. The fabrication is operated by VTT Technical Research Centre of Finland and the measurements are performed at Royal Military College of Canada. 


\section{Acknowledgments}

I am particularly indebted to my supervisors Dr. Didier Vincent and Dr. Langis Roy for the unconditional support, valuable guidance and endless patience they generously provided during the course of this work.

I graciously thank Dr. Barry Syrett for accepting to be my co-supervisor at Carleton University for the last three months and being present at my defense.

I would like to express my special thanks of gratitude to Dr. Joey R. Bray for his guidance during this thesis and allowing me to perform the measurements in Royal Military College of Canada. His valuable feedback and enthusiasm for research made my doctoral study a very enjoyable and rich experience.

A very significant thanks to Dr. Atif Shamim at King Abdullah University of Science and Technology for his financial assistance with the fabrication of the circulators.

I am grateful to PICON Odile, JACOB Arne, AMAYA Rony, FERRARI Philippe and BAYARD Bernard, who serve on my thesis examination committee for their time and efforts. Their assistance can never be appreciated enough.

I wish to express my sincere thanks to all staff of Laboratory LT2C and the department of electronics at Carleton University, for providing me with all the necessary facilities for the research.

Finally I would also like to thank my parents YANG Chengzhang and LIU Yingxiu 
and my wife LIN Zhuo, who support me to finish this thesis within the limited time. 


\section{Table of Contents}

Abstract $\quad$ v

Acknowledgments viii

Table of Contents $\quad$ x

List of Tables $\quad$ xv

List of Figures $\quad$ xvi

1 Introduction 1

2 Current state of rf ferrite devices $\quad 6$

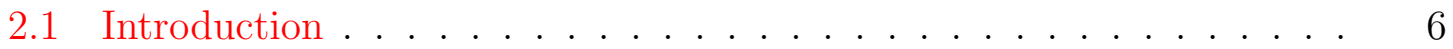

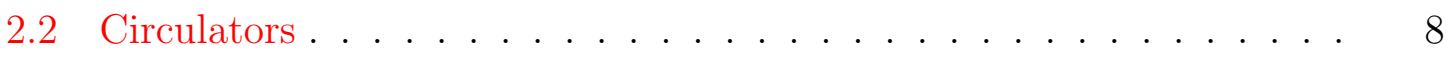

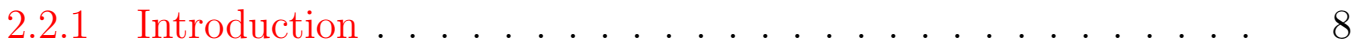

2.2.2 Main characteristics of a circulator . . . . . . . . . . . 11

2.2.3 Different types of circulator . . . . . . . . . . . . . . 14

2.2.4 Different conceptions of circulator . . . . . . . . . . . . 17

2.2.5 Circulators based on LTCC technology . . . . . . . . . . . 27

2.2 .6 Applications of circulators . . . . . . . . . . . . . . . 29

2.3 Isolators . . . . . . . . . . . . . . . . . . . . . . . . . . . 30 
2.3.1 Introduction . . . . . . . . . . . . . . . . . . . 30

2.3.2 Different conceptions of ferrite isolators . . . . . . . . . . . 31

2.3.3 Applications of isolator . . . . . . . . . . . . . . . . 33

2.4 Ferrite switch . . . . . . . . . . . . . . . . . . . 35

2.5 Ferrite phase shifters . . . . . . . . . . . . . . 35

2.5.1 Introduction . . . . . . . . . . . . . . . 35

2.5.2 Different conceptions of ferrite phase shifters . . . . . . . . . 36

2.6 Conclusion . . . . . . . . . . . . . . . . . . . . . . 38

3 Magnetic materials $\quad 41$

3.1 Introduction . . . . . . . . . . . . . . . . . . 41

3.2 Classes of magnetic materials . . . . . . . . . . . . . . . 42

3.2.1 Diamagnetism . . . . . . . . . . . . . . . . . 43

3.2.2 Paramagnetism . . . . . . . . . . . . . . . . 44

3.2.3 Ferromagnetism . . . . . . . . . . . . . . . 45

3.2.4 Antiferromagnetism ................ 46

3.2.5 Ferrimagnetism . . . . . . . . . . . . . 47

3.3 Ferrite materials . . . . . . . . . . . . . . . . . . . 47

3.3.1 Introduction . . . . . . . . . . . . . . . . . 47

3.3.2 Different types of ferrite . . . . . . . . . . . . . . . . 49

3.4 Basic properties of ferrite materials . . . . . . . . . . . . 50

3.4.1 Models of permeability tensor . . . . . . . . . . . . . 50

3.4.2 Demagnetization factors .............. 60

3.4.3 Effect of loss . . . . . . . . . . . . . . . 61

3.4.4 Hysteresis loop . . . . . . . . . . . . . . . . . . . 62

3.4.5 Magnetization $M_{s} \ldots \ldots \ldots$. . . . . . . . . 65 
3.4.6 Curie Temperature $T_{c} \ldots \ldots \ldots \ldots \ldots \ldots$

3.4 .7 Linewidth $\Delta H \quad \ldots \ldots \ldots \ldots \ldots \ldots \ldots$

3.4.8 Effective linewidth $\Delta H_{\text {eff }} \ldots \ldots \ldots \ldots \ldots \ldots \ldots$

3.4.9 Spin wave linewidth $\Delta H_{k} \ldots \ldots \ldots \ldots$

3.4.10 Permittivity and loss tangent $\ldots \ldots \ldots \ldots \ldots 6$

3.5 Ferrite LTCC . . . . . . . . . . . . . . . . . . . . . . . 68

3.5 .1 Introduction . . . . . . . . . . . . . . . 68

3.5.2 Characterization of ESL $40012 \ldots \ldots \ldots$. . . . . . . 69

3.5.3 Rf behavior of saturated ferrite . . . . . . . . . 75

3.5.4 Rf behavior of partially magnetized ferrite . . . . . . . 79

3.5.5 Conventional LTCC fabrication process . . . . . . . . . . 81

3.5.6 Recent ferrite LTCC research works . . . . . . . . . . . . . 82

3.6 Conclusion . . . . . . . . . . . . . . . . . . . . . . . . . . . . . 84

4 Modeling and realizations of circulators $\quad 87$

4.1 Introduction . . . . . . . . . . . . . . . . . . 87

4.2 CPW on ferrite LTCC . . . . . . . . . . . . . . . 88

4.2 .1 Introduction . . . . . . . . . . . . . . . . 88

4.2.2 Demagnetized ferrite $H_{i}=0 \ldots \ldots \ldots \ldots$

4.2 .3 Ferrite biased transversely . . . . . . . . . . . . . . 89

4.2.4 Prototype of a tunable attenuator . . . . . . . . . . . . . 91

4.3 Different structures of circulators . . . . . . . . . . . . . . 93

4.3 .1 Stripline circulator . . . . . . . . . . . . . . 94

4.3.2 Microstrip circulator . . . . . . . . . . . . . . . 95

4.3 .3 Coplanar circulator . . . . . . . . . . . . . . . . . 96

4.4 Theory of stripline junction circulators $\ldots \ldots \ldots \ldots$ 
4.5 Ferrite LTCC circulator with external magnet . . . . . . . . . . . 104

4.5 .1 Theory and design . . . . . . . . . . . . . . . . 104

4.5 .2 Measurement . . . . . . . . . . . . . . . . . . . . . 109

4.6 Ferrite LTCC circulator with integrated winding . . . . . . . . . 110

4.6 .1 Bias winding design . . . . . . . . . . . . . . . . 111

4.6.2 Y-junction edge-guided circulator design . . . . . . . . . 116

4.6 .3 Measurements . . . . . . . . . . . . . . . . . . . . . . . 123

4.6.4 Retro Analysis . . . . . . . . . . . . . . . . . 133

4.6.5 Power Capability IP3 . . . . . . . . . . . . . . . . 136

4.6 .6 Comparison and conclusion . . . . . . . . . . . . . . 139

4.7 Coplanar ferrite LTCC circulators . . . . . . . . . . . . . . . 140

4.7.1 Introduction . . . . . . . . . . . . . . . 140

4.7.2 Design of coplanar circulators . . . . . . . . . . . . . 140

4.7 .3 Measurements . . . . . . . . . . . . . . . . . . . . . . . . . 141

4.8 Wideband circulator by stacking two circulators . . . . . . . . . 144

4.8 .1 Introduction . . . . . . . . . . . . . . . . . . . 144

4.8.2 Theory and design . . . . . . . . . . . . . . . 145

4.8 .3 Simulation . . . . . . . . . . . . . . . . . . . . 147

4.9 Conclusion . . . . . . . . . . . . . . . . . . . . . . . . . . . . . . . . . . . . . . 149

5 Summary, conclusions and perspectives 152

5.1 Summary . . . . . . . . . . . . . . . . . . . 152

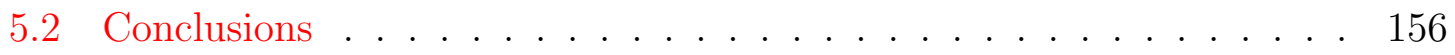

5.3 Perspectives . . . . . . . . . . . . . . . . 157

$\begin{array}{ll}\text { List of References } & 158\end{array}$ 


\section{List of Tables}

2.1 Comparison of main characteristics of current state of circulators . . . 16

2.2 Performance of different LTCC circulators . . . . . . . . . . . . . . . 29

4.1 Compare of circulators realized with LTCC technology . . . . . . . . 149 


\section{List of Figures}

2.1 Circulator separates the transmission and reception signals . . . . 8

2.2 Circulator consists of three ports oriented by $120^{\circ} \ldots$. . . . . . 10

2.3 Different shapes of circulator's center conductor . . . . . . . . . . 10

2.4 JQL Electronics: a. Coaxial circulator; b. Drop-in circulator; c. Surface Mount circulator; d. Microstrip circulator; e. High Power Waveguide circulator $[1] \ldots \ldots$. . . . . . . . . . . 14

2.5 Principle construction of a phase shift circulator [2] . . . . . . 18

2.6 Principle construction of a Faraday rotation circulator [3] . . . . . . 19

2.7 Principle construction of a ring circulator: $\mathrm{T}$ is reciprocal T-junction and PS is non-reciprocal phase shifter [4] . . . . . . . . . . 21

2.8 Principle construction of a stripline junction circulator [5] . . . . . 22

2.9 Standing wave pattern of a junction circulator: a. unmagnetized b. magnetized below resonance c. magnetized above resonance [2] . . . 23

2.10 Principle construction of an edge guided circulator . . . . . . . . . . 24

2.11 Principle construction of a lumped element circulator [6] . . . . . 25 
2.12 Self-biased circulator at $K_{u}$ band: (a) Cross section view of the junction resonator and quarter-wave microstrip line (b) Hysteresis loops of magnetically oriented M-type strontium hexaferrite. Perp refers to the measurement performed with the external field perpendicular to the sample surface, and par to the measurement with the field

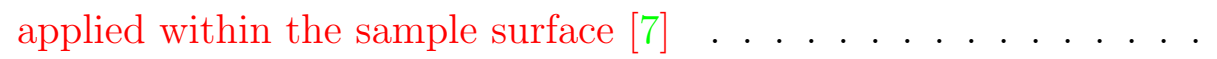

2.13 Non-reciprocal response based on (a) ferromagnetic media, and (b) angular-momentum-biased metamaterials. In ferromagnetic media, an external static magnetic bias (B) splits degenerate atomic states with opposite spin. Similarly, an externally applied angularmomentum vector splits counter-rotating degenerate modes of an azimuthally-symmetric ring, enabling nonreciprocity [8] . . . . .

2.14 Circulator used as duplexer . . . . . . . . . . . . . . . .

2.15 Combining networks for radio links: (a) solution based on filters; (b) solution based on circulators $[2] \ldots \ldots$. . . . . . . .

2.16 Two possible configurations of resonance isolator: (a) E-plane ferrite

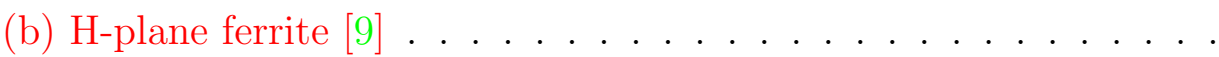

2.17 Geometry and electric fields of a field displacement isolator [9] . . .

2.18 Diagram of an isolator by adding a matched load to one of a circula-

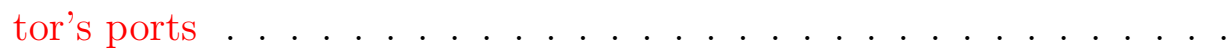

2.19 Diagram of a ferrite switch based on circulator . . . . . . . . . .

2.20 Nonreciprocal Faraday rotation phase shifter $[9]$. . . . . . . . .

2.21 Geometry of a latched phase shifter (a) using a ferrite toroid (b) using

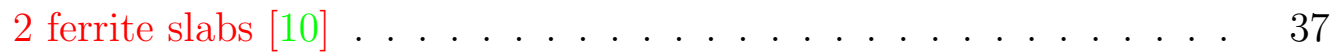

2.22 Reggia-Spencer phase shifter $[10] \ldots \ldots$. . . . . . . . . . . 38

3.1 Atomic and magnetic behaviour of diamagnetism [11] . . . . . . . . 43 
3.2 Atomic and magnetic behaviour of paramagnetism [11] . . . . . 45

3.3 Atomic and magnetic behaviour of ferromagnetism [11] . . . . . 46

3.4 Atomic and magnetic behaviour of antiferromagnetism [11] . . . . . 47

3.5 Atomic and magnetic behaviour of ferrimagnetism [11] . . . . . . 48

3.6 A spining electron: Spin magnetic dipole moment $(\vec{m})$ and angular

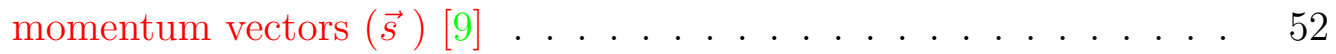

3.7 Geometric relation of different elements of permeability tensor . . . 55

3.8 Demagnetization factors of some simple shapes [12] . . . . . . . 61

3.9 An example of magnetic hysteresis loop [13] . . . . . . . . . . 63

3.10 Definition of the linewidth $\Delta H[9] \ldots \ldots$. . . . . . . . . . 66

3.11 Definition of the effective linewidth $\Delta H_{\text {eff }}[14] \ldots \ldots$. . . . . . 67

3.12 Definition of the spin wave linewidth $\Delta H_{k}[14] \ldots \ldots$. . . . . . . 68

3.13 Photo of ESL 40012 ferrite tape ('green' tape) . . . . . . . . . . . . 69

3.14 LTCC (a) solenoid transformer unit cell cross section and (b) toroid

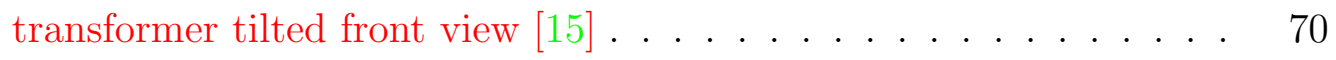

3.15 Measured hysteresis curves from the LTCC transformers [15] . . . . 70

3.16 B-H curve (first magnetization) used in EM simulator . . . . . . . . 71

3.17 Attenuation constant in terms of frequency . . . . . . . . . . . . 72

3.18 Major magnetization curves of the ESL 40012 sample at different sample temperatures, magnetic polarization $\mathrm{B}(\mathrm{T})$ versus magnetic field $\mathrm{H}(\mathrm{A} / \mathrm{m})$. The maximum magnetic field was $5 \mathrm{kA} / \mathrm{m}$ for all

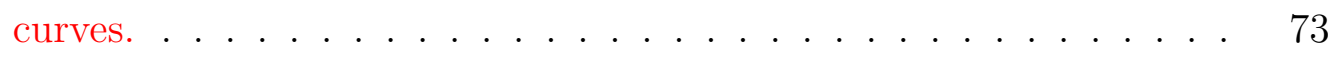

3.19 The VSM (Vibrating Sample Magnetometer) measurement performed by laboratory LT2C shows that the magnetization saturation $\mu_{0} M_{s} \operatorname{loses} 50 \%$ at $100^{\circ} \mathrm{C} \ldots \ldots \ldots$. . . . . . . . . 74

$3.20 \mu^{\prime}$ and $\kappa^{\prime}$ in terms of $\sigma \ldots \ldots \ldots \ldots 76$ 
$3.21 \mu^{\prime \prime}$ and $\kappa^{\prime \prime}$ in terms of $\sigma \ldots \ldots \ldots \ldots \ldots \ldots \ldots \ldots$

$3.22 \mu_{e f f}$ in terms of $\sigma \ldots \ldots \ldots \ldots \ldots \ldots \ldots \ldots \ldots$

$3.23 \kappa$ on $\mu$ in terms of $\sigma \ldots \ldots \ldots \ldots \ldots \ldots$

$3.24 \kappa$ and $\mu$ in terms of internal magnetization $M \ldots \ldots \ldots$

$3.25 \frac{\kappa}{\mu}$ and $\mu_{\text {eff }}$ in terms of internal magnetization $M \ldots \ldots . \ldots 81$

3.26 Typical multilayer ceramic substrate manufacturing process [16] . . 82

4.1 Design of a CPW on ferrite LTCC . . . . . . . . . . . . 88

4.2 Photo of a fabricated CPW on ferrite LTCC . . . . . . . . . . 89

4.3 Measurement configuration of the CPW with an external electromagnet 89

4.4 Measured S-parameters when ferrite is totally demagnetized . . . . 90

4.5 Simulated S-parameters when ferrite is totally demagnetized . . . . 90

4.6a Comparison of S21 and S12 between simulation and measurement when ferrite is biased transversely with internal magnetic field

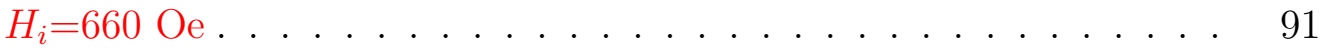

4.6b Comparison of S11 and S22 between simulation and measurement when ferrite is biased transversely with internal magnetic field

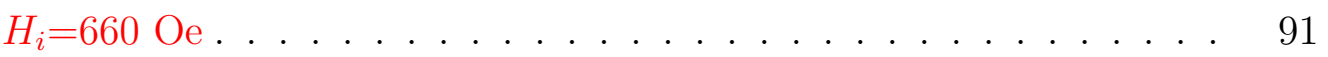

4.7 Measured resonance frequencies under different bias fields . . . . . 92

4.8 Comparison of measured and calculated resonance frequencies under different bias fields . . . . . . . . . . . . . . . . 99 93

4.9 Prototype of a tunable attenuator with windings in series . . . . . 93

4.10 Principle construction of a stripline junction circulator: (1) two metal grounds; (2,3) center conductor; (4) ferrite disk; (5) V is the coupling angle; (6) $\mathrm{u}$ is the height of the ferrite disk; $(7) H_{i}$ is the bias field; (8) $\mathrm{d}$ is the thickness of conductors; (9) $\mathrm{R}$ radius of the center conductor [5] 94

4.11 Principle construction of a microstrip junction circulator [17] . . . 96 
4.12 Top view of the signal line and the ground plane of a hexagonal circulator with CPW structure $[18] \ldots$. . . . . . . . . . . . 97

4.13 Circulator coordinate reference . . . . . . . . . . . . . . 98

4.14 Stripline circulator configuration . . . . . . . . . . . . 105

4.15 Calculated S-parameters in Matlab . . . . . . . . . . . 106

4.16 Parameters of an edge-guided circulator . . . . . . . . . . . . 107

4.17 Edge-guided circulator's 3D design . . . . . . . . . . . . . 108

4.18 Simulated S-parameters in HFSS when port 3 is matched: a circulator

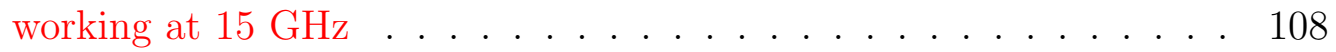

4.19 The measurement of the CPW verifies the calibration and demonstrates that the loss below $10 \mathrm{GHz}$ is strong . . . . . . . . . . . 109

4.20 The measurement of the circulator shows the working frequency is 15 $\mathrm{GHz} \ldots \ldots \ldots \ldots 110$

4.21a The de winding consists of 4 coils below the circulator and 2 coils above it . . . . . . . . . . . . . . . . . 111

4.21b Hexagonal coil dimensions . . . . . . . . . . . . . . . . . . . . . . 112

4.21c 2D conceptual sketch of the biasing magnetic flux . . . . . . . . . 113

4.22a Magneto-static simulation of winding in ferrite LTCC with a B-H curve: Bi along $\mathrm{Z}$ axis . . . . . . . . . . . . . . . . . . . . 113

4.22b Magneto-static simulation of winding in ferrite LTCC with a B-H curve: Bi along X axis on layer 6 (circulator inner conductor) . . . 114

4.22c Magneto-static simulation of winding in ferrite LTCC with a B-H curve: Bi along X axis on layer 9 . . . . . . . . . . . . . . . . 115

4.23 Edge-guided hexagonal circulator 3-D design for LTCC implementation 117 
4.24 The simulated S-parameters in Matlab by employing Green and Sandy's partially magnetized tensor model when $\mu \mathrm{M}=200 \mathrm{mT}$ and $\mathrm{R}=1.79 \mathrm{~mm} \ldots \ldots \ldots \ldots \ldots$

4.25 The simulated S-parameters in Matlab with Green and Sandy's partially magnetized tensor model under different internal magnetization

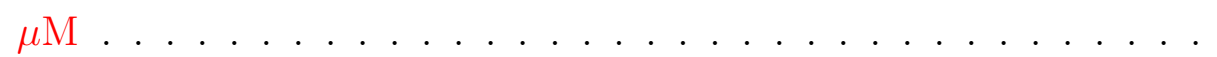

4.26 The circulator's working frequency in terms of the internal magnetization $\mu \mathrm{M} \ldots \ldots \ldots \ldots$

4.27 Transition from CPW transmission line to stripline . . . . . . . . . 121

4.28a Magnetic bias model in HFSS . . . . . . . . . . . . . . . . . . 122

4.28b Simulated S-parameters in HFSS and Matlab . . . . . . . . . . . . . 124

4.29 Prototypes of the ferrite LTCC circulator . . . . . . . . . . . . 125

4.30 S-parameters measurement configuration . . . . . . . . . . . . 125

4.31 CPW transmission line measurement: S-parameters . . . . . . . . 126

4.32 Measured S-parameters: Circulator without current . . . . . . . . 126

4.33 Measured S-parameters with current: S21 and S12 in terms of current 127

4.34a Comparison of measurement and HFSS: S21 and S12 . . . . . . . 128

4.34b Comparison of measurement and HFSS: S11 and S22 . . . . . . . 128

4.34c Circulator with an opposite direction of $300 \mathrm{~mA}$ dc current: Sparameters . . . . . . . . . . . . . . . . . . 129

4.35 Measurement probe station photo . . . . . . . . . . . . 129

4.36a Magneto-static simulation of the magnets in ferrite LTCC with a B-H curve: Bi along Z axis . . . . . . . . . . . . . . . . . . . . . . . . 130

4.36b Magneto-static simulation of the magnets in ferrite LTCC with a B-H curve: Bi along $\mathrm{X}$ axis . . . . . . . . . . . . . . . . . . 130

4.37a S-parameters with magnets: S21 and S12 in terms of current . . . . 131 
4.37b S-parameters with magnets: S11 and S22 in terms of current . . . . 131

4.37c Isolation and transmission curves when windings with the current is biased that opposes the magnetic fields of the magnet . . . . . . . . 133

$4.37 \mathrm{~d} \mu_{e f f}$ in terms of magnetization saturation $\mu_{0} M_{s}$ with $\mu M=0.2 T$ when the ferrite is partially magnetized using the model of Green

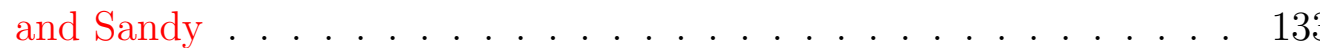

4.38a Magnetic bias model in CST . . . . . . . . . . . . . . . . . 134

4.38b Comparison of measured and simulated S21 and S12 by CST when

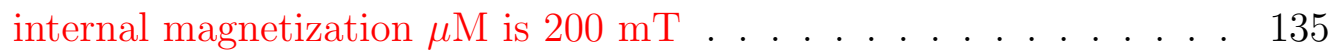

4.38c Comparison of measured and simulated S11 and S22 by CST when

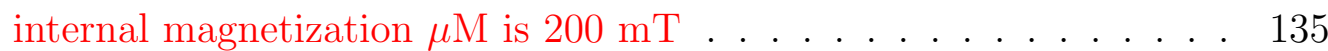

4.38d Simulated circulator's frequency variation (S11 in dB) in terms of

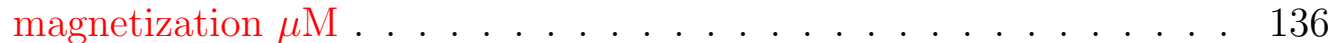

4.39 IP3 measurement details . . . . . . . . . . . . . . . . . . 137

4.40 Swept-Power IMD Test when current $\mathrm{I}=0 \mathrm{~mA} \quad \ldots . . . . .137$

4.41 Swept-Power IMD Test when current $\mathrm{I}=350 \mathrm{~mA} \quad \ldots . . . . . . \quad$.

4.42 Swept-Power IMD Test when current I=430 mA . . . . . . . . . . . 139

4.43 Comparison of circulators realized by ferrite LTCC and conventional LTCC technology . . . . . . . . . . . . . . . . . . 139

4.44 Design of a coplanar circulator (top view) [17] . . . . . . . . . . . . 141

4.45 Fabricated CPW and coplanar circulators operating at different fre-

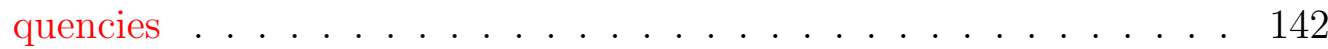

4.46 Measured S-parameters: coplanar circulator operating at $18 \mathrm{GHz}$. . 143

4.47 Measured S-parameters: coplanar circulator operating at $22 \mathrm{GHz}$. . 143

4.48 Measured S-parameters: coplanar circulator operating at $27 \mathrm{GHz}$. . 144

4.49a Design of a wideband circulator by stacking two circulators . . . . . 145 
4.49b Ferrite LTCC layers configuration . . . . . . . . . . . . . . . 146

4.50a Distribution of magnetic field $H_{i}$ in ferrite LTCC . . . . . . . . 147

4.50b Internal bias field $B_{i}$ in terms of Z . . . . . . . . . . . . . . . 148

4.51 S-parameters by co-simulation in HFSS and Maxwell 3D . . . . . . 149 


\section{Chapter 1}

\section{Introduction}

In recent years, multi-standard systems such as GSM, UMTS, LTE, 4G/5G have been employed in cellular radio handsets. In addition, a carrier aggregation (CA) service has been started and cognitive radio terminals with reconfigurable rf front-end circuit are in the developmental stage for the next-generation of communication systems. Since mobile handsets using the multi-standard systems are handling a large number

of frequency bands, the rf circuits of the handsets have been becoming more and more complex with many signal paths corresponding to various frequency bands. On the other hand, the mobile data transfer rates have been enhanced and the frequency bands have been extending to higher frequencies. Therefore a miniaturized and tunable rf front-end architecture for frequency division duplexing (FDD) system is very effective for simplifying the circuit configuration [19]. In wireless radar applications, circulator seems to be the most suitable microwave device as a duplexer, because the transmission and reception signals have the same frequency, hence they cannot be separated by frequency filtering. Essentially, circulator allows duplex (bi-directional) communication when a single path is used. It avoids interaction between the receiver and the transmitter, permitting them to use a shared antenna.

In order to miniaturize rf circuits, we introduce the Low Temperature Co-fired 
Ceramics (LTCC) technology. Since LTCC has a 3D design ability, therefore the size of the circuits could be greatly reduced. Furthermore, as the fired temperature is below $1000^{\circ} \mathrm{C}$, LTCC could apply low loss metals (Au, Ag, Pt etc) as conductors. Consequently, compared with applications based on other materials like resin, LTCC applications have relatively low losses and high microwave performance. In addition, the thermal expansion coefficient of LTCC applications is competitive compared with resin materials and other ceramic materials. More important, LTCC could be combined with many other materials with different characteristics, therefore various types of components could be integrated and built into LTCC substrates. For these reasons, LTCC is regarded as one of the promising future technologies for the integration of components and substrates for rf applications [16]. Application of LTCC technology is now widely spread and is a good candidate for SoP (System-on-Package) system. Its presence is well established in high frequency applications [20,21], sensor applications, component packaging, chemical reactors and bio-medical applications [22].

Unfortunately, conventional LTCC technology does not work for ferrite devices which require non-reciprocal materials. Some researchers have demonstrated a hybrid approach to realize circulators by sintering traditional LTCC substrate and ferrimagnetic materials together [23-25]. This hybrid method creates an internal cavity using conventional LTCC technology where a piece of ferrimagnetic material is put in and then sintered (cofired together). The disadvantages of this approach are: 1 . the ferrimagnetic material should be able to be co-fired (compatible) with the non-magnetic ceramic LTCC substrate; 2. an external magnet is necessary to generate the static magnetic bias; 3 . the demagnetizing field cannot be avoided.

Meanwhile, a new technology, ferrite LTCC technology, which is originally used to design inductors, now could be used to design high frequency tunable devices with integrated winding, such as tunable antenna [26-28], tunable filter [29] and tunable 
phase-shifter [30]. Ferrite LTCC substrate is a ferrite material, therefore we can embed a winding into the substrate, creating the dc magnetic bias from the inside. By using this elegant solution, we can remove the external magnets (or electromagnets) and avoid the demagnetization fields. In addition to eliminating the demagnetization effect, the required bias field strength can be further reduced by operating the ferrite in the partially magnetized state instead of saturation state [28].

Having this new technology, we present this thesis to firstly investigate original circulator design fully embedded in ferrite LTCC substrates, then study the performance of a ferrite LTCC circulator with integrated winding and finally investigate coplanar circulators and a wide-band circulator. For the ferrite LTCC circulator with integrated winding, the controllability of the cores bias field makes this prototype device multifunctional: when no bias field is present, it behaves as a power splitter; with bias, it behaves as a circulator and the direction of circulation can be switched by reversing the direction of the DC current; finally, when used with small external magnets, the DC current can be used to change the circulators working frequency. The proposed device could be used in many applications. For instance, when used between a transmitter and a pair of antennas, the circulator/splitter could be used to control the radiation pattern: it could either feed the two antennas at the same time, or power only one of them for a chosen direction or polarization. Based on this principle, a more complex feed network could also be realized by applying more than one circulator/splitter to achieve pattern and polarization diversity. Switchable circulators can also be used to control the direction of propagation through a transmitarray.

Chapter 2 is about the current state of ferrite rf devices. Firstly, the basics of circulators will be introduced, followed by the main characteristics of a circulator such as the transmission, isolation, bandwidth, power capability, etc. Then, different 
types and conceptions of circulators are presented, including the phase-shift circulator, Faraday rotation circulator, ring circulator, junction circulator, the circulators realized by LTCC technology etc. The applications of circulators such as decoupling, combining and isolating are also proposed. Since ferrite isolator is an important ferrite device, various conceptions of ferrite isolators are also presented. Phase shifter, which is widely used in phased array antennas, is discussed in this chapter along with several conceptions. Finally, other ferrite devices, like ferrite switch is briefly described.

The objective of Chapter 3 is to present the magnetic materials. In general, there are diamagnetic, paramagnetic, ferromagnetic, antiferromagnetic and ferrimagnetic materials. Usually, ferrimagnetic materials, or ferrite is employed in microwave devices because of their high resistivity (not conducting), low dielectric loss and reasonable dielectric constant. We will present the characterization of ferrite materials by explaining the dielectric loss, hysteresis loop, magnetization saturation, Curie temperature, linewidth (including effective linewidth and spin wave linewidth) etc. Different permeability tensor theories for saturated, partially magnetized and demagnetized ferrite are presented. In particular, the ferrite LTCC tape ESL 40012, which is used in this thesis, is described, along with the properties under different magnetized conditions (saturated and partially magnetized). Since ferrite LTCC's fabrication process is not accessible to the authors, only the conventional LTCC process is demonstrated.

The modelization and realization of ferrite LTCC circulators is located in Chapter 4. This chapter starts by studying a CPW transmission line on the ferrite LTCC substrate. This work allows to have a first manipulation of designing rf devices on this substrate. Then we briefly introduce different structures of circulators including the stripline, microstrip and coplanar structure. The stripline structure is chosen in this thesis to realize circulators because the two metal grounds of the stripline 
provide a rf shielding while allowing the magnetostatic bias field into the ferrite core. The theory of a stripline junction circulator is presented in detail with mathematical calculations in Matlab. Then, two ferrite LTCC circulators are realized: the first is a stripline circulator with external magnets. This work demonstrates, for the first time, the feasibility of a circulator on this substrate. The second is a ferrite LTCC circulator with integrated winding which magnetizes the ferrite from the inside. This circulator is multifunctional. When the winding is energized by a current, the device operates as a dynamic circulator in which the circulation direction can be changed by switching the direction of the current. Besides, the proposed device can even operate as a power splitter by removing the bias current. Then several coplanar circulators are also realized to find the maximum frequency which the substrate could support. Finally a wide band circulator by stacking two circulators with different operating frequencies is proposed and simulated. 


\section{Chapter 2}

\section{Current state of $\mathrm{rf}$ ferrite devices}

\section{$2.1 \quad$ Introduction}

Microwave passive non-reciprocal devices such as circulators, switches, isolators and phase shifters use an important type of material: ferrite. Ferrites allow electromagnetic waves to penetrate and then the waves interact with the internal magnetic moments within the ferrite. Besides, ferrites are magnetic ceramic materials instead of magnetic metals which are conductors, therefore ferrites are favorable as microwave device substrates. The microwave propagation in ferrite devices is controlled by a dc magnetic field which could be static or tunable. The ferrite devices can be linear or nonlinear, and reciprocal or nonreciprocal. Nowadays, microwave systems widely use ferrite components and various types of ferrite materials are employed according to the design requirements. A number of devices have been developed across a wide range of microwave spectrum for various power levels and are now commercially available over the last 50 years. As an example, adjustable phase shifters are used in the shipboard Aegis systems and ground-based Patriot where the agile beam phased array radars and beam steering components are employed. By changing the applied dc magnetic field (hence internal magnetization), a rf within the ferrite phase shifters 
could obtain different phase shifts. These devices are based on gyromagnetic effects of ferrites leading to the properties of microwave phase shift. Circulators based on ferrites are desired in radar systems to be duplexers which isolate transmitters from receivers. In addition, selective filter banks also employ circulators as switches.

Studies of microwave ferrite devices suggest that one or more of the following effects could be used to explain their behaviors [31]: "Faraday rotation - the rotation of the plane of polarization of a microwave as it propagates through a ferrite in the direction of the magnetization. ferromagnetic resonance - the strong absorption that can occur when an elliptically polarized rf magnetic field is perpendicular to the direction of magnetization. field displacement - the displacement of the field distribution transverse to the direction of propagation resulting in more or less field in the ferrite region. nonlinear effects - at higher power levels amplification and frequency doubling are possible and subsidiary losses can occur. spin waves - shortwavelength waves of magnetization that can propagate at any angle with respect to the direction of magnetization. If the wavelength of such a wave is comparable to the dimensions of the ferrite sample, it is called a magnetostatic wave (MSW)" [31].

A strong understanding of the microwave circuit theory, electromagnetic theory and magnetic materials is necessary in the development of ferrite devices [32]. Although many research efforts have been taken in this area, the demands for miniaturized size, broader fractional bandwidths, higher operating frequencies, and reduced costs are still expected to meet [31] leading to many challenges to be addressed. Our knowledge about the microwave circuit theory has been advanced by the study of ferrite devices [31]. 


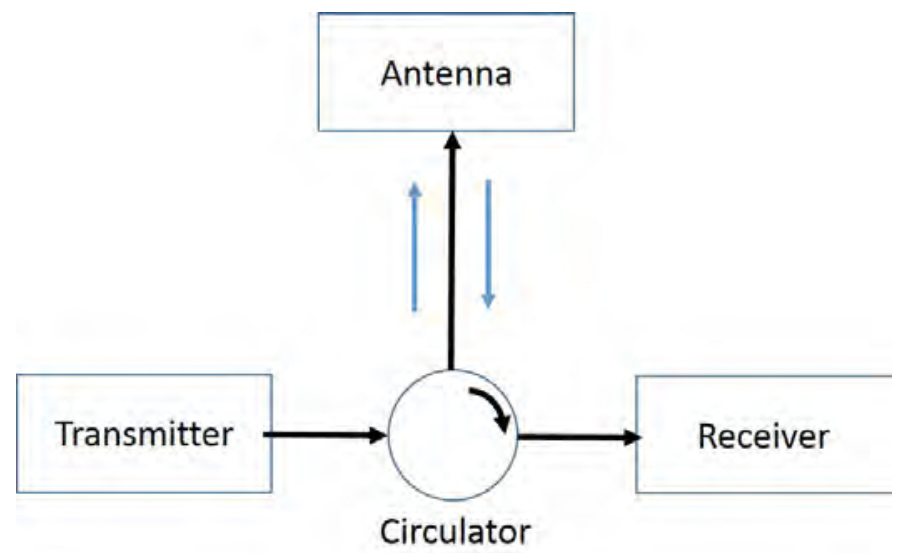

Figure 2.1: Circulator separates the transmission and reception signals

\subsection{Circulators}

\subsubsection{Introduction}

Currently most telecommunication systems operating at microwave frequencies are based on the technology of rf circuits. Passive non-reciprocal components such as phase shifters, isolators, circulators, tunable antennas are widely used in wireless telecommunication systems like radar system, cellphone base station, satellite communication system, etc. Essentially, circulators provide traffic between different signal ports in one direction and not in the other. Therefore circulators are employed in microwave applications as duplexers to separate the transmission and reception signals when only one shared antenna (and one carrier frequency) is used (see Figure 2.1). A three-port circulator can also be used as an isolator by connecting one of the cir-

culator's ports to a matched load which absorbs the reflected energy. This sort of isolator serves to protect a source against any reflected energy, especially in microwave applications with high power.

The brief history of circulators is [33]: In the early 1950's, we saw the first commercial microwave circulator. Faraday rotation circulator was one of the most famous 
circulators [34]. There were later resonance isolators [34] and differential phase shift circulators [35], which were easy to be constructed and had high power-handling capabilities. Then, field-displacement devices came into our sight [36], along with junction circulators in the early 1960's. An important moment in 1962, H. Bosma developed a theory to explain the operation of a Y-junction circulator $[5,37]$. The theory employs the Green function approach to calculate the distribution of rf fields in a stripline Y-junction circulator. Later, a theory explaining the rotation of the rf standing-wave in a circulator's ferrite disk was proposed by Fay and Comstock. It describes two rotating waves under the biasing field [38]. The "continuous frequency tracking" conditions for a wide-band junction microstrip circulator were then developed by Wu and Rosenbaum [39]. Afterwards, many researchers discussed how to realize low loss and wide-band circulators [40-42]. Self-biased waveguide and microstrip circulators were then developed utilizing hexagonal ferrite by Weiss, Watson, and Dionne [43]. Recently, in 2015, the modeling and characterization of a self-biased circulator working at $41 \mathrm{GHz}$ was demonstrated in [44]. In addition, circulator with SIW (Substrate Integrated Waveguide) based on nano-composite ferrite was also proposed [45]. Circulators, as passive non-reciprocal devices known for a long time are still under research to improve the performance and achieve the miniaturization.

Circulator could have different structures, like stripline, microstrip, coplanar structure etc. Taking a stripline circulator as an example, it usually consists of three access ports oriented at $120^{\circ}$ (see Figure 2.2) [46] to each other around a center conductor. Various shapes (see Figure 2.3) are possible to be the center conductor [47]. The material that creates the non-reciprocity, often a ferrite disk, is separated by the center conductor.

The performance of the circulator shown in Figure 2.2 could be expressed by the 


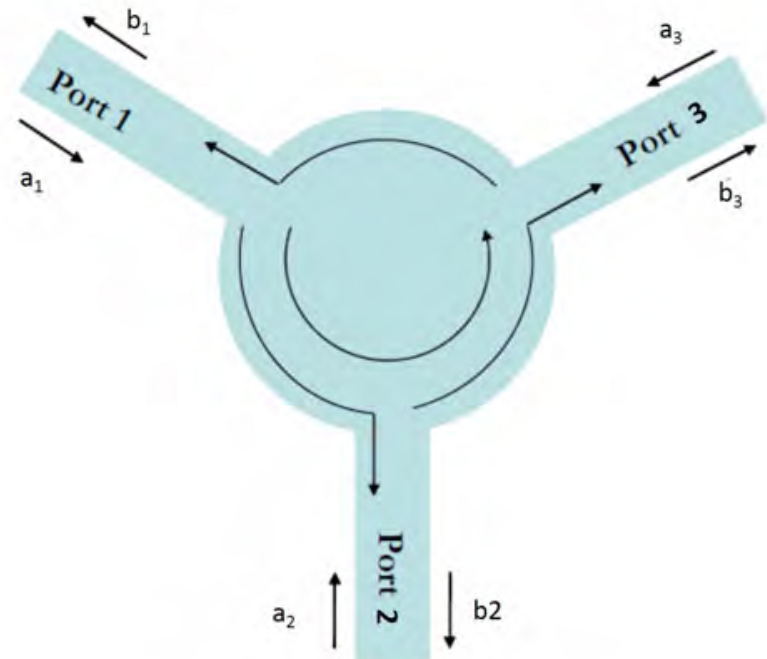

Figure 2.2: Circulator consists of three ports oriented by $120^{\circ}$

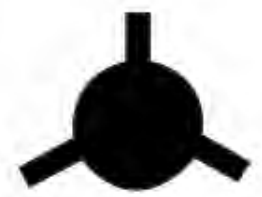

a

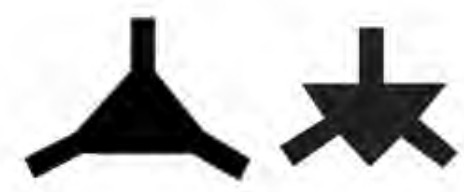

b
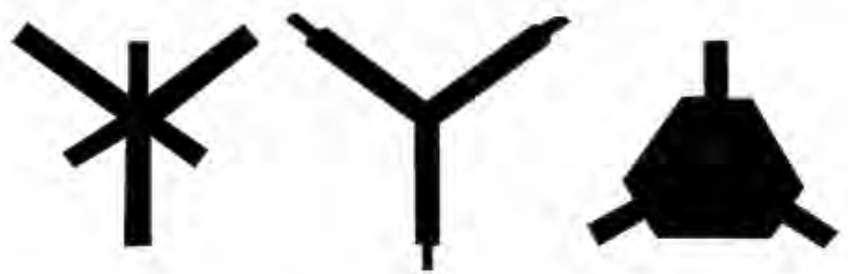

Figure 2.3: Different shapes of circulator's center conductor

matrix of S-parameters (Equation (2.1)):

$$
b=S \times a \Rightarrow\left(\begin{array}{c}
b_{1} \\
b_{2} \\
b_{3}
\end{array}\right)=\left(\begin{array}{lll}
S_{11} & S_{12} & S_{13} \\
S_{21} & S_{22} & S_{23} \\
S_{31} & S_{32} & S_{33}
\end{array}\right) \times\left(\begin{array}{l}
a_{1} \\
a_{2} \\
a_{3}
\end{array}\right)
$$


The parameters S11, S22 and S33 are the reflection coefficients at port 1, 2 and 3, while the parameters S21, S32 and S13 are the transmission parameters between the ports and correspond to the insertion loss of the device. Finally, the parameters S12, S23 and S31 are the coefficients of inverse transmission and correspond to the isolation between the ports (see Figure 2.2). The signal flows in an anti-clockwise movement in this case and the signal sequence is as follows: $1 \rightarrow 2 \rightarrow 3 \rightarrow 1$. The S-parameter matrix is given, in the ideal case (without losses), by Equation (2.2):

$$
S=\left(\begin{array}{lll}
0 & 0 & 1 \\
1 & 0 & 0 \\
0 & 1 & 0
\end{array}\right)
$$

\subsubsection{Main characteristics of a circulator}

A circulator's performance is mainly characterized by the isolation, insertion loss, bandwidth, power capability, temperature and in some cases, frequency tunability.

\section{Isolation}

Circulator, as an important component in microwave applications, is required that the device has a high isolation level. Isolation measures the separation of signal levels on adjacent ports of a device. High isolation (in $\mathrm{dB}$ ) value leads to a low interference of the signal between one port and the other. The value of isolation relates to the Voltage Standing Wave Ratio (VSWR). Taking a circulator whose port 3 is connected to a matched load as an example: if a poor match (e.g. VSWR=2) at port 3 is observed, the expected isolation is below $10 \mathrm{~dB}$, but if a good match load is used as the termination, the VSWR match could be improved to 1.1 which 
corresponds to an isolation over $20 \mathrm{~dB}$. However, if impedance match changes at all the three ports, active intelligence (compensation of matching) could be employed. This remains to be explored by future work.

\section{Insertion loss}

Insertion loss is another important parameter for circulators. Generally, the insertion loss is significant at high frequencies. Accordingly, a low insertion loss is desired to avoid wasting precious power. The typical value of the insertion loss for a circulator working at $\mathrm{X}$ band is $<0.5 \mathrm{~dB}$.

\section{Bandwidth}

The bandwidth is the frequency range where it ensures a value of desired reflection, isolation and insertion loss. The fractional bandwidth is commonly used which is the bandwidth of a device divided by the center operating frequency. Commercialized circulators usually have a fractional bandwidth over $3 \%$ of the working frequency. Some circulators could even cover one or several bands such as the band $\mathrm{X}, \mathrm{Ku}, \mathrm{K}$, Ka, etc.

\section{Power capability}

The power capability is an issue of the linearity of circulators. Circulators are considered to be linear devices and usually work under P1 dB compression point or IP3 point. High power is usually necessary for radar systems where high energy radars/antennas are used. The power capability varies according to different manufactures and applications, typically 2 - 250 Watts. More power can be achieved at the expense of device's size and cooling systems. As an example, a single junction 
isolator (based on a circulator) with an internal $10 \mathrm{~W}$ load capability could be manufactured by MECA Electronics [48]. However, for safe heat transfer and derating, the recommended maximum power that the device could operate with is $2 \mathrm{~W}$. Meanwhile, arming an external load would make heat transfer to an external heat sink, consequently higher isolator power level could be achieved easily at the cost of the size.

\section{Temperature}

The saturation magnetization $M_{s}$ of ferrite material is a strong function of temperature, decreasing as temperature increases. This effect can be understood by noting that the vibrational energy of an atom increases with temperature, making it more difficult to align all the magnetic dipoles [9]. At a high enough temperature (threshold), the thermal energy is greater than the energy supplied by the internal magnetic field, and a zero net magnetization results. This temperature is called the Curie temperature, $T_{C}[9,49]$. The typical maximum operating temperature is $75^{\circ} \mathrm{C}$ according to JQL Electronics Inc [1].

\section{Frequency tunability}

Circulator's frequency tunability is usually achieved by changing the static external magnetic bias field provided by a bulky electromagnet. Conventional circulators work under saturated state, so the external magnet need to overcome the demagnetization field. In order to increase the internal magnetic field by $0.1 \mathrm{~T}$, extra $80 \mathrm{KA} / \mathrm{m}$ magnetic $\mathrm{H}$ field is required, leading to the difficulty of designing a compact tunable device. Recently, researchers have demonstrated that an integrated winding in ferrite LTCC ESL 40012 could easily change the internal magnetic field from 0 to $0.4 \mathrm{~T}$ using a $\mathrm{H}$ field less than $2.5 \mathrm{KA} / \mathrm{m}$ under partially magnetized state [15]. This idea 
could be used to design compact tunable devices [26-30]..

\subsubsection{Different types of circulator}

Commercialized circulators could be classified into different categories according to their connections (see Figure $2.4[1])$ :

- Coaxial circulator

- Drop-in circulator

- Surface-mount circulator

- Microstrip circulator

- Waveguide circulator
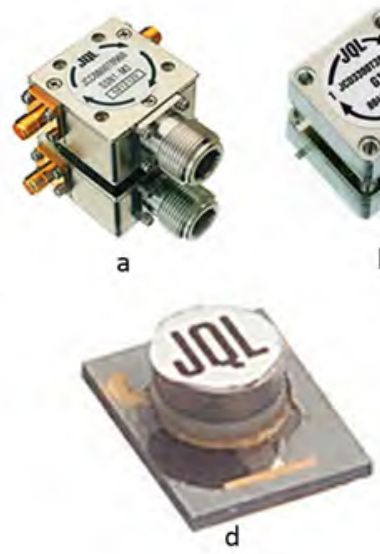

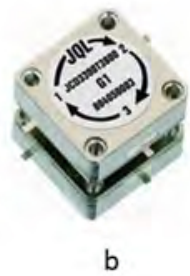

b
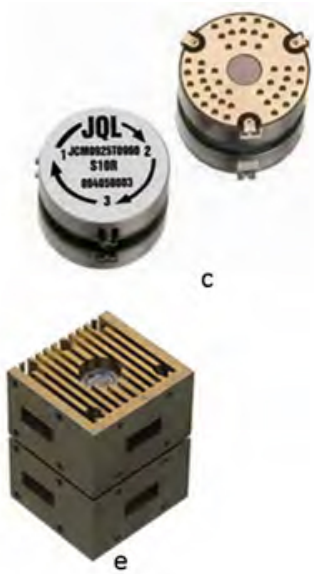

Figure 2.4: JQL Electronics: a. Coaxial circulator; b. Drop-in circulator; c. Surface Mount circulator; d. Microstrip circulator; e. High Power Waveguide circulator [1]

Typically these circulators have a broad selection of frequencies and bandwidths. They are designed for military, spatial and commercial applications. The power 
capability generally ranges from several single watts to mega-watts with isolation above $18 \mathrm{~dB}$ per junction and low insertion loss (below $0.5 \mathrm{~dB}$ ). They often have SMA or N connectors.

Table 2.1 shows the main performance of current commercialized circulators at $\mathrm{X}$ band. High power circulators are usually realized with waveguides and they can be easily found in military radar system who requires high radiation power. However, high power handling circulators come with large sizes. Compact circulators like surface-mount circulators are miniaturized at the expense of power capability. 


\subsubsection{Different conceptions of circulator}

Circulators can be realized using different conceptions which are briefly described below [2]:

- Phase shift circulator

- Circulator based on Faraday rotation

- Ring circulator

- Junction circulator

- Edge-guided mode circulator

- Lumped-element circulator

- Self-biased circulator

- Magnetic-free circulator

\section{Phase shift circulator}

Phase shift circulators are commonly applied in high power (up to Mega-Watt) radar systems [35] [50]. As an example, the conventional 4-port differential phase shift circulator is based on a rectangular waveguide, where thin ferrite slabs are loaded and transversely-magnetized (see Figure 2.5) [2].

Differential phase shift circulator is nonreciprocal and based on a differential phase shift of $90^{\circ}$ between a magic $\mathrm{T}$ (whose output signals have an equal phase) and a 3$\mathrm{dB}$ coupler (whose output signals have a $90^{\circ}$ phase difference) in the device. A wave goes into port 1, then the magic $\mathrm{T}$ splits the wave into two waves which have equal 


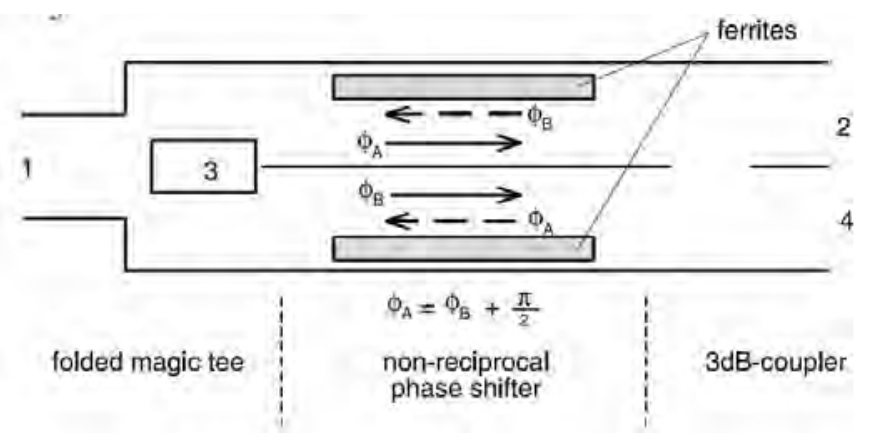

Figure 2.5: Principle construction of a phase shift circulator [2]

energy and phase. After that, the two waves enter the phase shifter which is nonreciprocal. The first wave is shifted by $\Phi_{A}$, and the second wave by $\Phi_{B}$, where there is a difference of $90^{\circ}$ between $\Phi_{A}$ and $\Phi_{B}$. In the $3 \mathrm{~dB}$-hybrid, the two waves are split a second time and each wave has two equal parts, but this time, the 3 -dB hybrid leads a $90^{\circ}$ delay between two outputs. Therefore the waves cancel each other at port 4 while add each other at port 2. Similarly, a wave entering port 2 will be transmitted into port 3 and port 1 is isolated.

The geometry, magnetic losses, bias field magnetization, low power losses and high material temperature-stability (Curie temperature) performance should be considered when we design a high power phase shift circulator. The parameters of ferrite slabs, such as the thickness and length along with the permanent magnetic field are crucial to have a $90^{\circ}$ phase shift [50]. Phase shift circulators are bulky, especially those for high continuous wave $(\mathrm{CW})$ power applications.

\section{Circulator based on Faraday rotation}

The theory and design of a circulator employing a Faraday rotator is firstly discussed in [34]. These circulators rely on the Faraday rotation effect which is considered to be non-reciprocal $[3,51]$.

The diagram of a conventional four-port Faraday rotation circulator is shown 


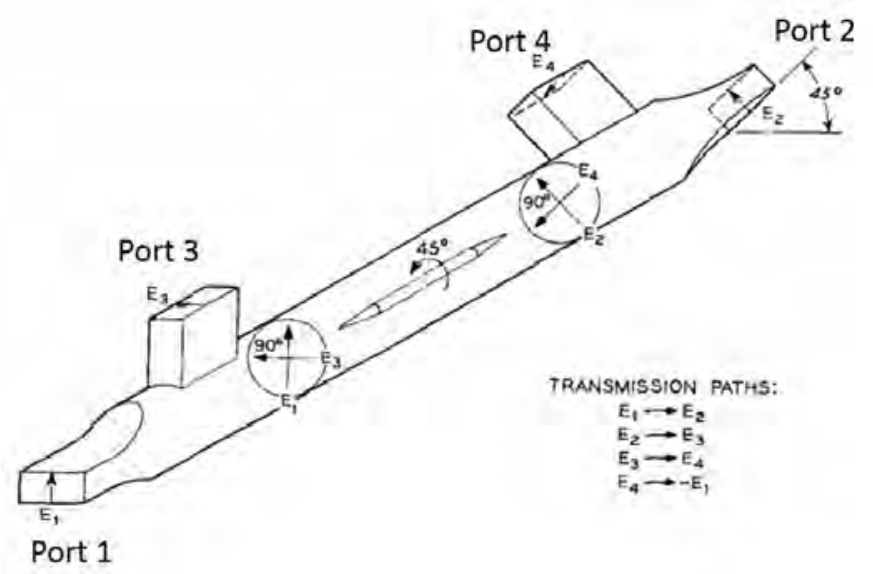

Figure 2.6: Principle construction of a Faraday rotation circulator [3]

in Figure 2.6 [3]. There are two transitions between the rectangular and circular waveguide at the two nodes of the circulator. A $T E_{10}$ wave is injected into the left port of the rectangular waveguide, then the first transition guides the wave into a round waveguide, meanwhile the mode becomes a linearly polarized $T E_{11}$ wave. A round ferrite rod is placed at the center of the waveguide and magnetized in the direction of the waveguide. Two circular rotating waves then obtained: one rotating clockwise, the other anti-clockwise when we look in the direction of propagation. the electron spins in the ferrite rod then interact with these two waves. One wave has the propagation speed $\mu_{+}$and the other has the $\mu_{-}$. If the two rotating waves are combined after they have traveled some distance, a linear $T E_{10}$-wave is formed a second time with some rotating degrees. With careful calculation of the biased magnetic field and the length of ferrite rod, a rotation of $45^{\circ}$ could be expected. Finally, the round waveguide is transformed into the rectangular waveguide via the second transition and forms the final output of the circulator (see Figure 2.6). Therefore all the input energy passes through exclusively from port 1 to port 2 . In a like manner energy entering port 2 passes exclusively to port 3, energy entering port 3 passes exclusively to port 4 , and 
energy entering port 4 passes exclusively to port 1.

Circulators based on Faraday rotation are usually built as isolators. There are only two ports: one input and one output. Resistive sheets are employed at the other ports in order to absorb the waves, hence avoiding reflected energy. Faraday rotation isolators could works at high frequencies [52] and nowadays as optical isolators of fibre cables [53].

\section{Ring circulator}

Ring circulator is barely used in practice and mainly studied in theory [2]. A rigorous theoretical analysis of the ring circulator concept based on a network model is discussed in [54] by Weiss. A conventional three-port ring circulator is shown in Figure 2.7 [4]. The design is symmetrical and it is composed of three reciprocal $\mathrm{T}$ junctions and three nonreciprocal phase shifters. In theory, by choosing appropriate phase shifts, such junctions could be assembled to be a perfect circulator. Accordingly, only a small amount of nonreciprocal phase shift and internal wave amplitude are required.

A wave goes into port 1 and then is split into two waves: one follows clockwise and the other anticlockwise. The nonreciprocal phase shifters then delay the two waves. The phase shifts are carefully chosen so that they cancel each other at port 3 and add up at port 2 forming a circulation. Ring circulators present competitive mechanical characteristics (weight and size), insertion loss and bandwidth. They are possibly employed in some unique applications with high-speed switching, special power capability, etc $[4,55,56]$. 


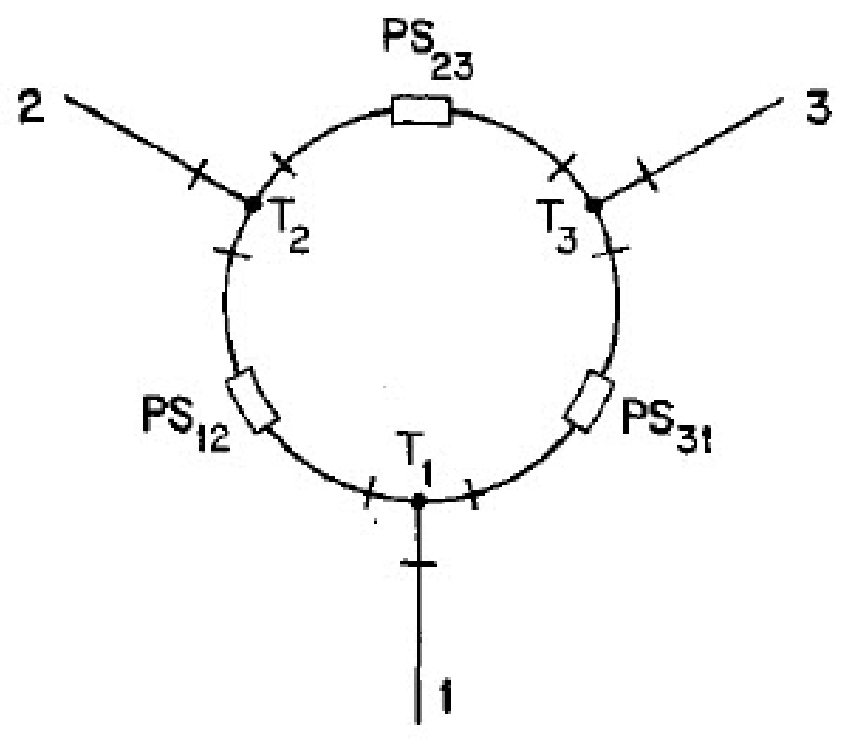

Figure 2.7: Principle construction of a ring circulator: $\mathrm{T}$ is reciprocal $\mathrm{T}$-junction and PS is non-reciprocal phase shifter [4]

\section{Junction circulator}

The theoretical approach to the three-port symmetrical circulator is presented in [57] for the most general waveguide case. The stripline Y-junction circulator has a simple geometrical arrangement which makes it fairly easy to be studied. This is a symmetrical structure, as shown in Figure 2.8 [5], where a ferrite disk is separated by the center conductor and the three access lines are spaced by $120^{\circ}$. Two metal ground planes then envelop the structure. The space between the two metal grounds surrounding the ferrite disk is occupied by dielectric materials. The ferrite material is magnetized perpendicularly to the plane of the disk by a static magnetic field created by two magnets located on both sides of the structure.

The idea of a Y-junction circulator with three ports was envisaged by Carlin (1954) [58] on the theoretical basis of the processing of the S-parameters matrix of a junction with three ports. However, the current form of Y-junction circulator 


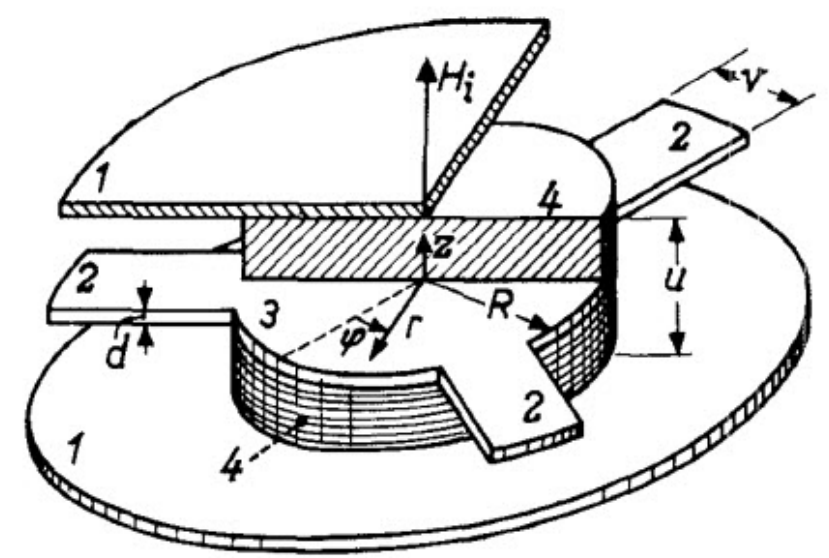

Figure 2.8: Principle construction of a stripline junction circulator [5]

and the analysis of three-port junction containing ferrites were performed by Bosma $(1962)[5,37]$ who gives general design equations for the approximate radius of ferrite, width of stripline, operating frequency etc. Then Fay and Comstock [38] explained the operation of the rf field pattern within a circular ferrite disk (see Figure 2.9) [2]: When the biasing magnetic field is not applied, a wave goes into port 1 and then is split into two waves: one follows clockwise and the other anticlockwise possessing the same propagation speed. A standing wave pattern is hence formed in the device, leading to the coupling to port 2 and port 3 . The device is therefore a power divider where half of the power goes into port 2, the other half port 3 (see Figure 2.9 a); When a biasing magnetic field is applied perpendicularly to the ferrite material, the interaction with the electron spin in the ferrite creates the propagation speed difference between the two rotating waves. The clockwise rotating wave possesses the propagation velocity $\gamma_{+}$, while the anti-clockwise rotating wave $\gamma_{-}$. Consequently, the rotation of the standing wave pattern is observed. The angle of rotation could be adjusted by the applied dc magnetic field. Specially, if the angle of anti-clock rotation is $30^{\circ}$, a circulator is obtained (see Figure $2.9 \mathrm{~b}$ ): port 3 is decoupled and the wave traverses from port 1 to port 2. Besides, if a higher biasing magnetic field is used, there is also 
another circulator's operation: the standing wave pattern is rotated towards clockwise by $30^{\circ}$, leading to the decoupling of port 2 and all energy passes from port 1 to port 3 (see Figure 2.9 c).

The first mode is called low $H_{i}$ (internal magnetic field) mode because the external static dc magnetic field is lower than the ferrite's resonance frequency. On the contrary, the second mode is called high $H_{i}$ for higher static magnetic field.

Triplate junction circulators could be designed as high $H_{i}$ circulators for the frequency range from $0.15 \mathrm{GHz}$ to about $2 \mathrm{GHz}$ to achieve potential low losses (small $\left.\mu^{\prime \prime}\right)$ or as low $H_{i}$ circulators for the frequency range from $1.5 \mathrm{GHz}$ up to $40 \mathrm{GHz}$ [2].
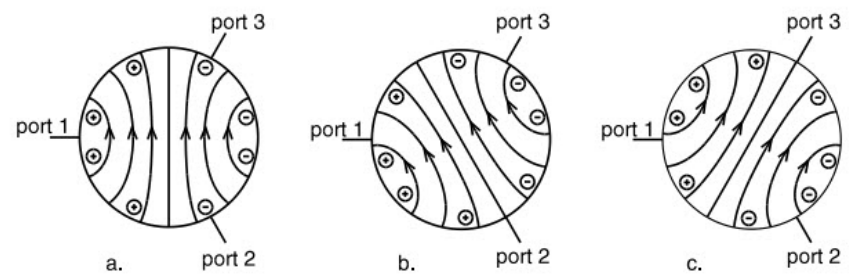

Figure 2.9: Standing wave pattern of a junction circulator: a. unmagnetized b. magnetized below resonance c. magnetized above resonance [2]

\section{Edge-guided mode circulator}

A complete study on edge guided circulators (EGC) is presented by De Santis and F. Pucci in 1975 [59]. The fundamental physical principles of edge guided wave (EGW) which underlie the EGC's operations are established in [59,60]. Edge-guided circulators are exploited to construct broad-band circulators. In addition, broad-band isolators (up to one octave) could also be realized using edge-guided effect [61].

This effect of edge guided wave is manifested by a suitably magnetized wide strip of a microstrip or stripline gyromagnetic line. Conventionally, in triplate or microstrip lines, the distribution of $\mathrm{rf}$ currents is on two edges of the center conductor. When a 
ferrite material is applied and biased perpendicularly to the line, the rf current leans against one edge of the inner conductor only for one propagation direction and on the other edge for the opposite direction. One of the most attractive characteristics of EGW is that it is extremely broad band [62].

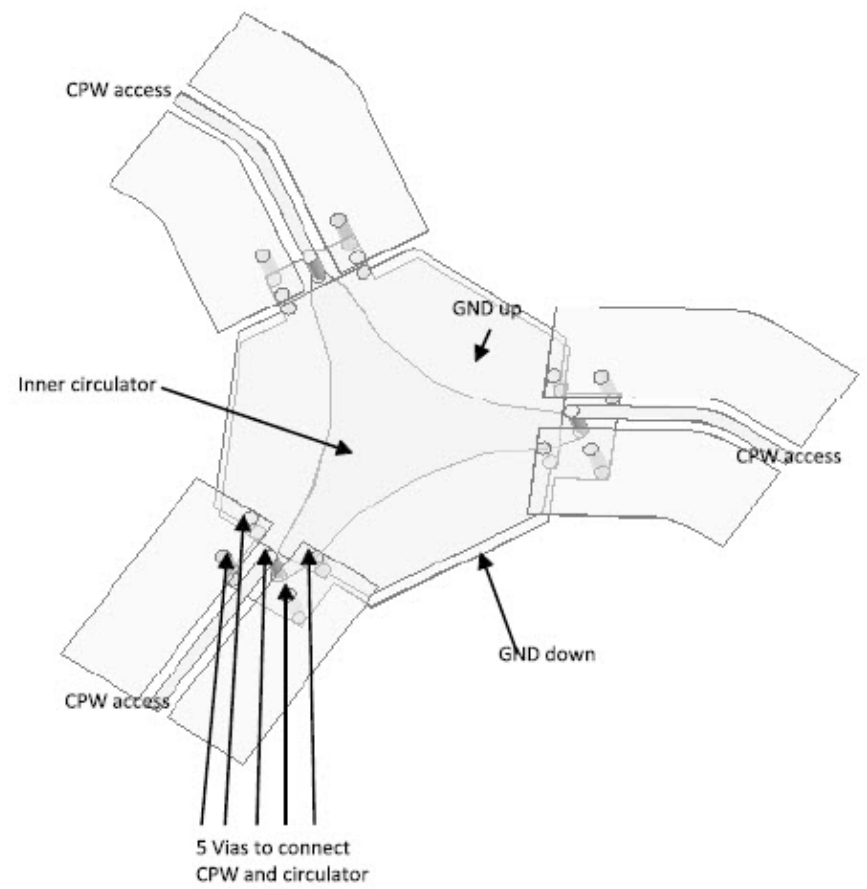

Figure 2.10: Principle construction of an edge guided circulator

Figure 2.10 shows an edge-guided mode circulator designed in this thesis. The center conductor has three smooth curves and the ferrite substrate is strongly magnetized perpendicular to the plane of the circulator. The input fields then focus on one edge of the center conductor and leave at the next port, making the third port isolated. 


\section{Lumped element circulator}

The circulators described before give a bulky construction when the operating frequency is low $(\mathrm{MHz})$. This is the reason why lumped element circulators are introduced [63]. The basic idea of a lumped-element circulator from the crossover ferrite circulator for VHF (Very High Frequency) and UHF (Ultra High Frequency) applications was established by Konishi in 1965 [64]. A mesh mechanism instead of an ordinary Y-stripline center conductor was proposed. The core of this circulator is a non-reciprocal ferrite crossover network junction. Figure 2.11 shows the principle design of such a junction: three conductors cross over each other and oriented by an angle of $120^{\circ}$. They are all connected to the same outer conductors at the other end. The inner conductors are isolated from each other. Two ferrite disks are deposited between the inner and outer conductors and magnetized by an external dc magnetic field $[64,65]$.

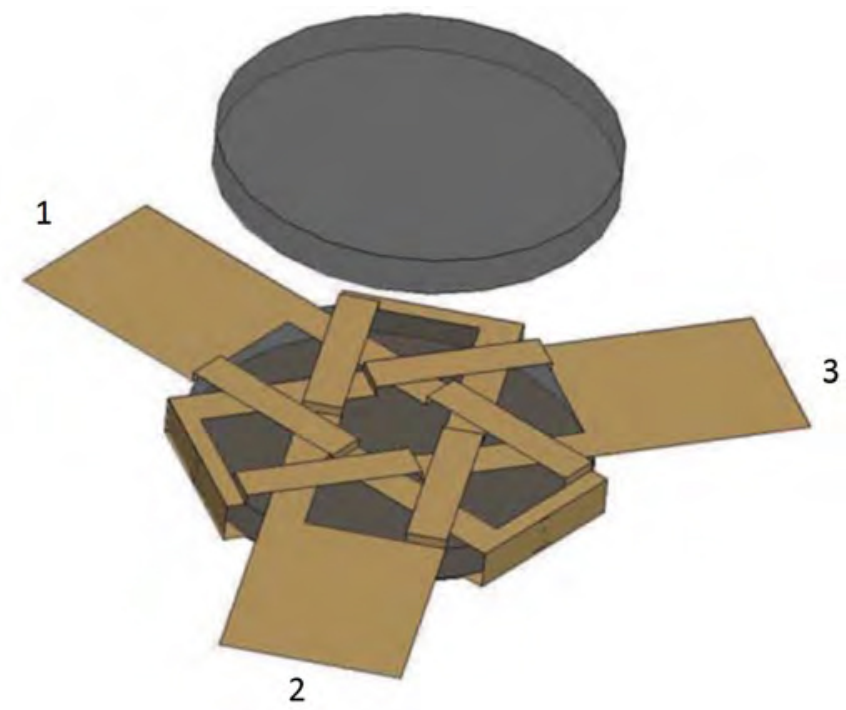

Figure 2.11: Principle construction of a lumped element circulator [6]

Since lumped-element circulator works for low frequencies and external lumped 
elements could be used for tuning, they could be used in tunable cellphone applications. Recently, a reconfigurable miniaturized circulator used in mobile handsets was proposed [66]. The tunable band is from $1.71 \mathrm{GHz}$ to $2.17 \mathrm{GHz}$, along with a small size $\left(\mathrm{L}^{*} \mathrm{~W}^{*} \mathrm{H}\right) 1.2 \mathrm{~mm} \times 1.0 \mathrm{~mm} \times 0.5 \mathrm{~mm}$. Their port impedances are arranged by matching circuits using varactors. Besides, two supplementary varactors are also used as compensation elements for the enlargement of the frequency bandwidth.

\section{Self-biased circulator}

Ferrite used for a millimeter-frequency circulator is required to have a large magnetization saturation and low dielectric and magnetic losses [67]. The circulators presented before need huge external magnet to bias the ferrite for millimeter applications over $30 \mathrm{GHz}$ [43]. However the large uniaxial anisotropy fields present in hexaferrites could be used to provide ferrimagnetic self-resonant frequencies above $40 \mathrm{GHz}$ in oriented materials when they are self-biased, i.e., behave as permanent magnets. This material property could be used to get rid of the external magnets, greatly reducing the overall circulator size $[33,43,67,68]$.

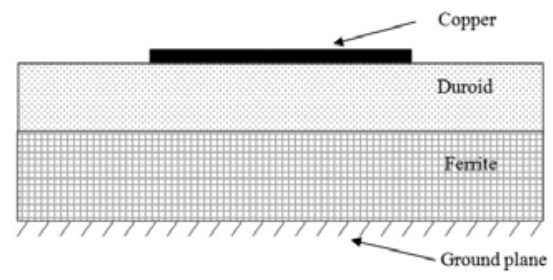

(a)

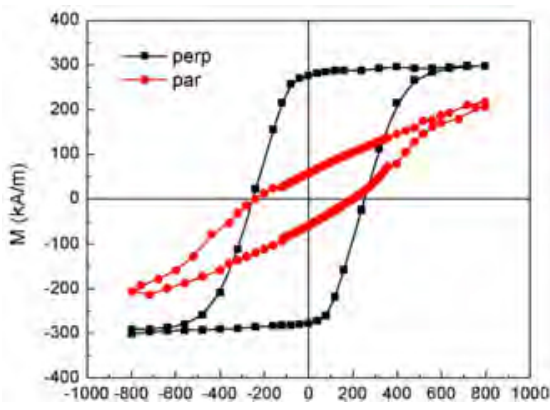

(b)

Figure 2.12: Self-biased circulator at $K_{u}$ band: (a) Cross section view of the junction resonator and quarter-wave microstrip line (b) Hysteresis loops of magnetically oriented M-type strontium hexaferrite. Perp refers to the measurement performed with the external field perpendicular to the sample surface, and par to the measurement with the field applied within the sample surface [7] 
Self-biased circulator designs are mainly based on the self-biased magnetic materials. The conception of this circulator which uses the microstrip structure is shown in Figure 2.12(a) and the hysteresis loops of magnetically oriented M-type strontium hexaferrite are shown in 2.12(b). Self-biased circulators usually work at high frequencies because of the high internal anisotropy field. The circulator presented in [44] has demonstrated a circulation at $41.4 \mathrm{GHz}$. However, recently a self-biased circulator working at $\mathrm{Ku}$ band is presented in [7] which demonstrates that the self-biased circulator can also operate below $30 \mathrm{GHz}$.

\section{Magnetic-free circulator}

A different approach to realize nonreciprocal microwave components and materials, based on biasing meta-molecules with the angular-momentum vector, has been presented in [8]. This method, theoretically introduced in [69], uses azimuthal spatiotemporal modulation to generate an effective biasing angular momentum vector, which can break time-reversal symmetry in a way analogous to magnetic bias, and can subsequently induce nonreciprocity, as envisioned in Figure 2.13. By using appropriate spatiotemporal modulation of a magnetic-free distributed-element resonating ring, an isolation over $47 \mathrm{~dB}$ is achieved while size is small [8]. However, the insertion loss is about $22 \mathrm{~dB}$ which is not competitive for a circulator working at $170 \mathrm{MHz}$.

\subsubsection{Circulators based on LTCC technology}

As we said before, LTCC technology is now widely spread. However, conventional LTCC tape is non-magnetic ceramic material, while non-reciprocal devices, like circulators, require magnetic material such as ferrite. In order to realize circulators on LTCC technology, researcher have developed a hybrid approach by cofiring ferrites 


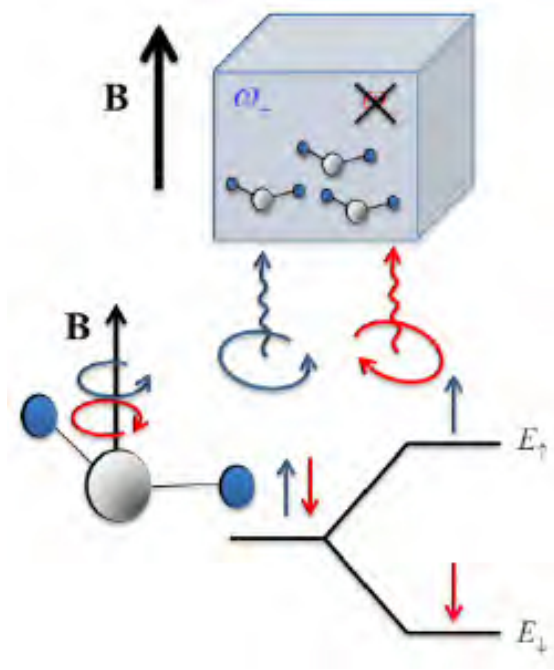

(a)

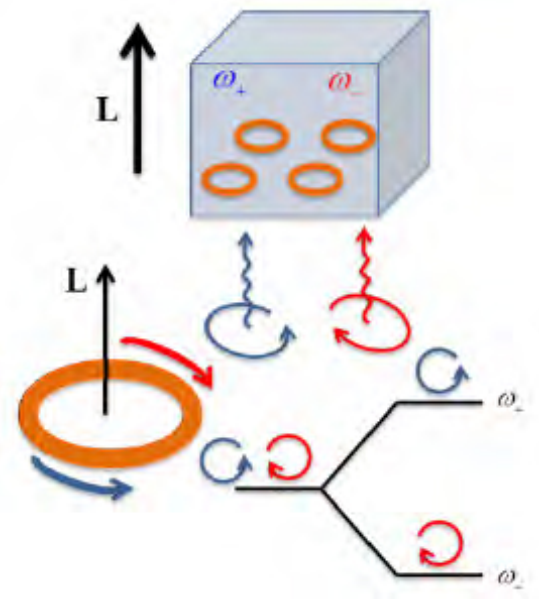

(b)

Figure 2.13: Non-reciprocal response based on (a) ferromagnetic media, and (b) angular-momentum-biased metamaterials. In ferromagnetic media, an external static magnetic bias (B) splits degenerate atomic states with opposite spin. Similarly, an externally applied angular-momentum vector splits counter-rotating degenerate modes of an azimuthally-symmetric ring, enabling nonreciprocity [8]

with non-magnetic ceramic LTCC tapes, as discussed in [23-25]. The main idea of these designs is to integrate ferrites into the conventional LTCC fabrication process to build circulators. The challenge in this development of LTCC circulators is the cofiring of laminated tapes that have different material compositions and subsequently different shrinkage rates, leading to defects, such as cracks and warpage in the fired module if care is not taken. Table 2.2 shows the performance of different LTCC circulators to date by cofiring ferrite and LTCC tape. However, the disadvantages of this approach are: 1 . the ferrimagnetic material should be able to be co-fired with conventional LTCC substrate; 2. an external magnet is still necessary to generate the static magnetic bias; 3 . the demagnetizing field cannot be avoided. In this thesis, we realize a circulator based on ferrite LTCC with integrated winding which magnetizes the ferrite from the inside. Therefore the external magnet is removed and we avoid the demagnetizing field [27,30]. Furthermore, the controllability of the bias 
Table 2.2: Performance of different LTCC circulators

\begin{tabular}{ccccccc}
\hline $\mathrm{F}(\mathrm{GHz})$ & ISO $(\mathrm{dB})$ & INS $(\mathrm{dB})$ & $\mathrm{P}(\mathrm{dBm})$ & Type & Size $(\mathrm{mm})$ & Reference \\
\hline \hline 7.4 & 25 & 1.7 & N/A & Microstrip & N/A & T. Jensen [24] \\
$15-17$ & 20 & 0.8 & N/A & Microstrip & $7.5^{*} 6$ & Jianhua Ji [23] \\
5.4 & 23 & 0.5 & 45 & Microstrip & N/A & R. Van Dijk [25] \\
$11.8-13.2$ & 22 & 1.25 & 42.8 & Microstrip & N/A & R. Van Dijk [25] \\
\hline
\end{tabular}

field makes the circulator multifunctional. We will present a study on this circulator with integrated winding in Chapter 4.

\subsubsection{Applications of circulators}

\section{Duplexer}

The most common application of a circulator is to separate $\mathrm{Tx}$ and $\mathrm{Rx}$ signals as shown in Figure 2.14. If one transmitter and one receiver are connected to a shared antenna, two high-performance (sharp) and expensive filters are required to avoid their interaction $[2,70,71]$, however, by employing a circulator for branching (duplexing), we only need an inexpensive filter at the receiver's input [2].

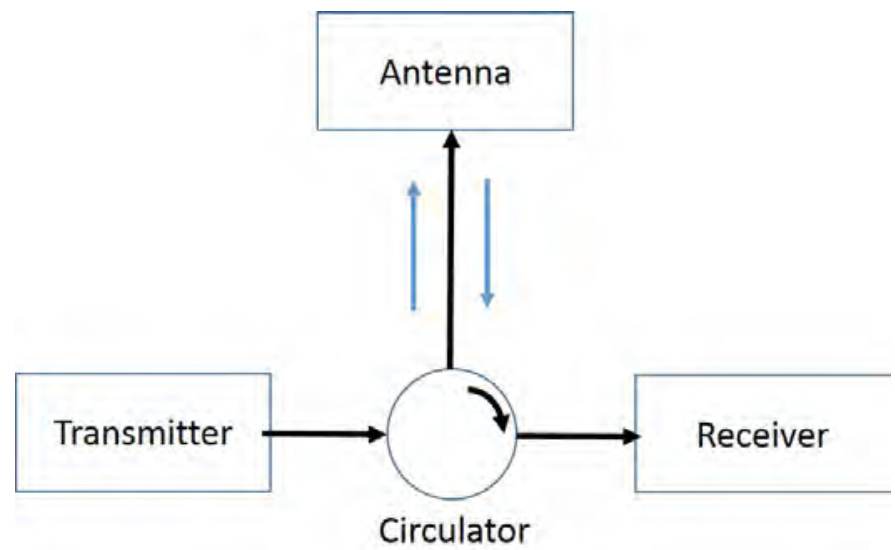

Figure 2.14: Circulator used as duplexer 


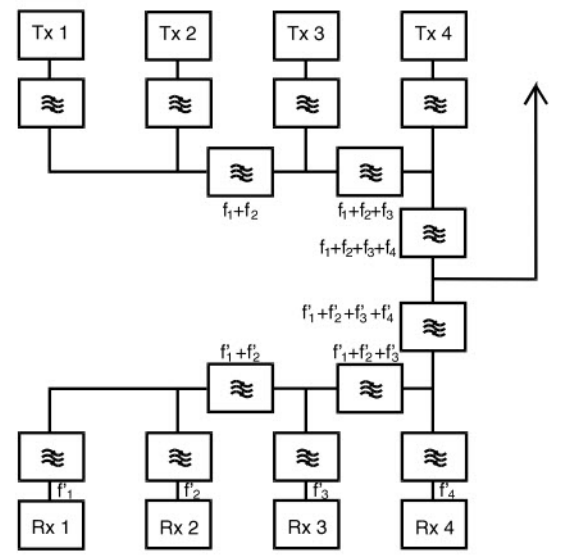

(a)

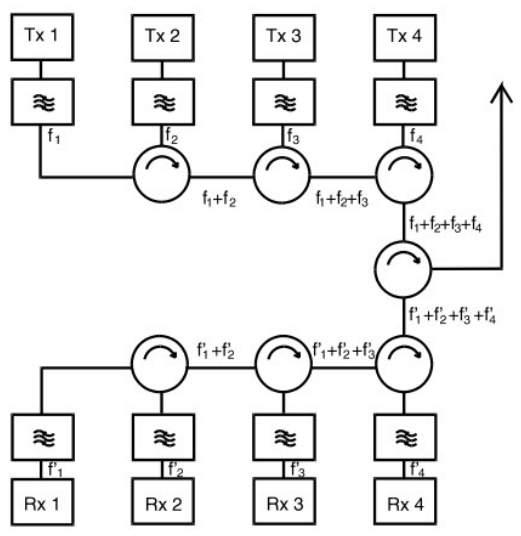

(b)

Figure 2.15: Combining networks for radio links: (a) solution based on filters; (b) solution based on circulators [2]

\section{Combiner}

Circulator can also be used as a combiner for more than one transmitter, for example, radio links. The combining of radio links turns to be challenging when the frequency is higher than $2 \mathrm{GHz}$ [2]. As an example, for a station with four transmitters and four receivers (see Figure 2.15(a)), a matrix of sharp filters are used to form the combiner. The loss of each filter leads to a relatively high total loss, especially for transmitter 1 and receiver 1 . In addition, the filters are not independent from each other, so that tuning one filter affects the others which makes it a difficult task. If some of the filters are replaced by circulators (see Figure 2.15(b)), the losses could be reduced and we can improve the independence between filters when tuning is performed [2]. However, this solution of circulator combining have stringent requirements for intermodulation. 


\subsection{Isolators}

\subsubsection{Introduction}

Isolator is one of the important ferrite devices. A rf isolator can be considered as a diode for rf energy, allowing signal to go through in one direction only. Isolator is a two-port device and the S-parameters for an ideal isolator have the form shown by Equation (2.3). When two ports are matched, the transmission is allowed only in the direction from port 1 to port 2 and blocked in the reverse direction. The isolator must be lossy because the matrix of S-parameters is not unitary. Besides, isolators are nonreciprocal components, so that the $[\mathrm{S}]$ is not symmetrical [9].

$$
[S]=\left[\begin{array}{ll}
0 & 0 \\
1 & 0
\end{array}\right]
$$

\subsubsection{Different conceptions of ferrite isolators}

\section{Resonance isolators}

A strong interaction with the ferrite material is expected when a circular polarized plane wave rotates in the same direction as the precessing moment of magnetic dipoles in a ferrite medium. However, a circularly polarized wave rotating in the opposite direction will have a weak interaction. This effect could be used to design isolators when the rf signal is near the gyromagnetic resonance of the ferrite.

In order to design a resonance isolator, Figures 2.16(a) and 2.16(b) show two possible configurations (E-plane and H-plane) of ferrite slab in a waveguide [9]. Eplane configuration is easy to be biased without the demagnetizing field. However the magnetic fields don't have a real circular polarization and the heat transfer from 


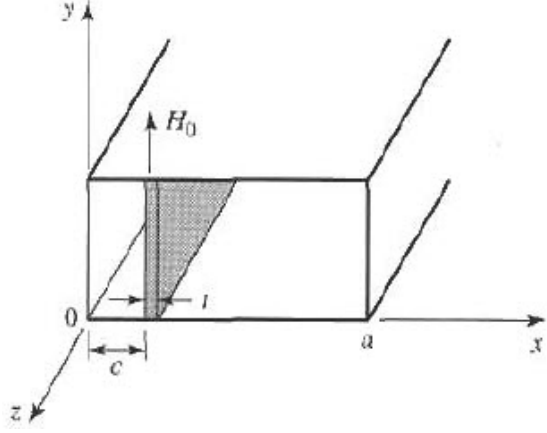

(a)

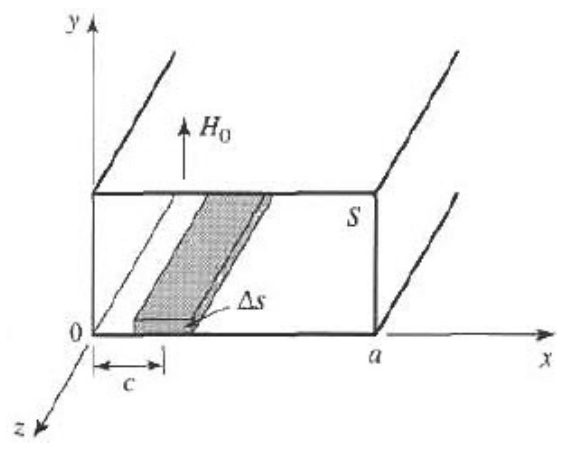

(b)

Figure 2.16: Two possible configurations of resonance isolator: (a) E-plane ferrite (b) H-plane ferrite [9]

the slab is poor. H-plane configuration allows a real circular polarization and better thermal properties, but it requires more bias field to overcome the demagnetizing field [9]. The main disadvantage of the resonance isolators is that the bandwidth is narrow.

\section{Field displacement isolator}

To improve the bandwidth and reduce the bias field, field displacement isolator could be used. There is quiet a difference of electric field distributions between the forward and reverse waves in a ferrite slab-loaded waveguide. Figure 2.17 shows that it is possible to make the electric field of the forward wave vanish, while the electric field of the reverse wave is made to be maximum at this same point (point $\mathrm{x}=\mathrm{c}+\mathrm{t}$ ). The reverse wave will be attenuated by a thin resistive sheet which is placed in this position. Meanwhile, the forward wave can go through without being affected $(\mathrm{c}+\mathrm{t}$ $<\mathrm{x}<$ a) [9]. The fractional bandwidth of field displacement isolators could be on the order of $10 \%$. In addition, a small bias field is needed because it works below the resonance point. 


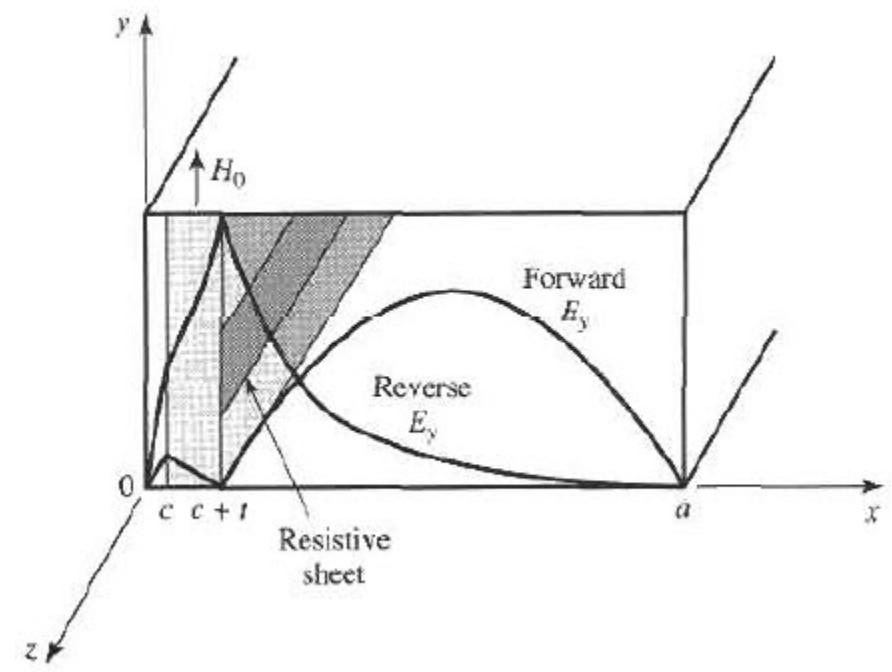

Figure 2.17: Geometry and electric fields of a field displacement isolator [9]

\section{Using a circulator}

As mentioned before, by adding a matched resistive load to one of a circulator's ports, a two-port isolator could be obtained (see Figure 2.18). If the circulator works under the resonance point, small bias field is sufficient to make this device work. Moreover, thermal transfer could also be improved because the power is absorbed externally [72].

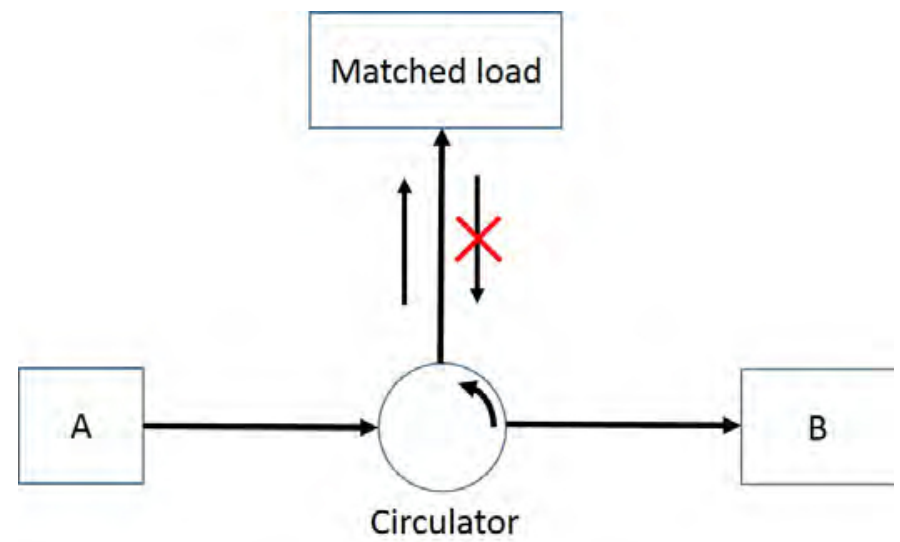

Figure 2.18: Diagram of an isolator by adding a matched load to one of a circulator's ports 


\subsubsection{Applications of isolator}

Isolators are mainly used for decoupling. The problem that power reflecting back to generators may lead to nonlinear effects such as the instability and frequency shifting [73]. To avoid this, an isolator between the generator and the load could be applied in order to attenuate only the reflected wave and hence protect the generator [2].

Besides, in applications where cascade amplifiers are used, the change of one stage's input impedance could affect others [73]. The stability is crucial for narrow band devices because high precision of input impedance is required. The solution is to use isolators between different amplifier stages, then they can be adjusted without interacting with the others. If one stage is unbalanced, the others will also be protected and not be overloaded. Besides, we can extend greatly the interval of re-tuning power transmitters by this way. As an example, tubes (IOT's) as final amplifiers isolators is commonly recommanded between the driver stages and inductive output for decoupling purpose [2].

Fading due to obstacles, for example, snow accumulation on the reflector of an antenna is a challenging problem in satellite communication in northern countries such as Finland [74]. Hence the transmitter is affected by impedance variations of the antenna. This can be avoided by employing an isolator between transmitter and the antenna.

\section{$2.4 \quad$ Ferrite switch}

Essentially, ferrite switch is a derivation of a three-port ferrite circulator (see Figure 2.19). In theory, wires thread a toroidal ferrite resonator, therefore, the direction of circulator's circulation could be controlled by energizing the wires with current pulses. The insertion loss is expected to be $0.15 \mathrm{~dB}$ per latching junction circulator, 
along with $20 \mathrm{~dB}$ return loss and isolation. The fractional bandwidth is usually over $5 \%$ to $10 \%$. A holding current is not required, since the switch is a latched device [75].

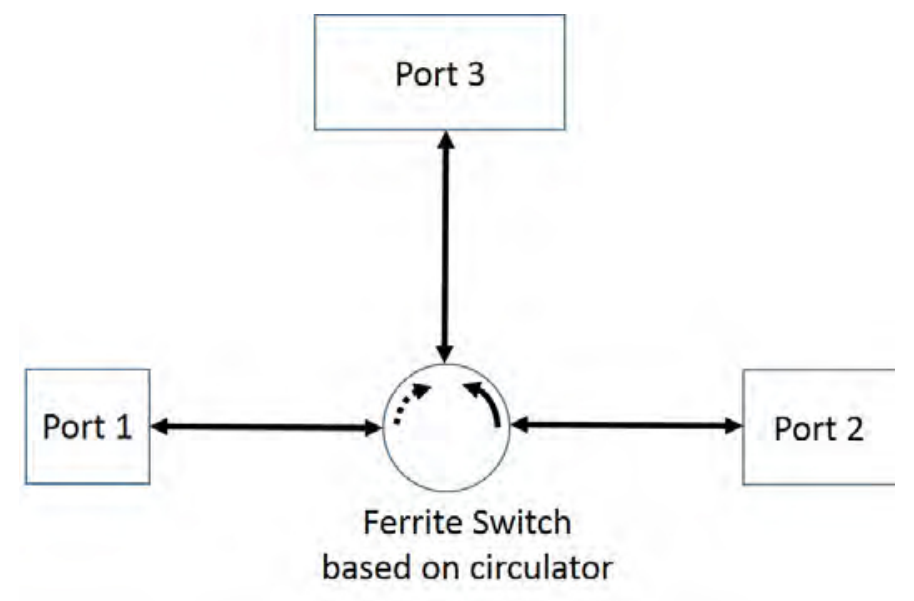

Figure 2.19: Diagram of a ferrite switch based on circulator

\subsection{Ferrite phase shifters}

\subsubsection{Introduction}

Phase shifter is among the most important applications based on ferrite materials. Basically, the ferrite phase shifter is a two-port device which provides different phase shifts by adjusting the external dc bias field applied to the ferrite. Currently, various types of phase shifters are available, either reciprocal (same phase shift in both directions) or nonreciprocal. They are in demand in phased array antenna systems where the steering-beam antennas are employed [9]. 


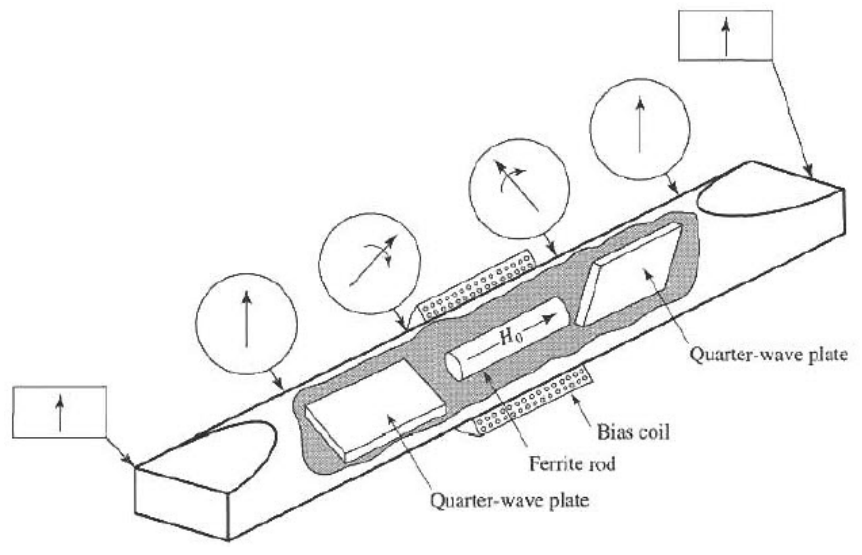

Figure 2.20: Nonreciprocal Faraday rotation phase shifter [9]

\subsubsection{Different conceptions of ferrite phase shifters}

\section{Nonreciprocal/reciprocal Faraday rotation phase shifter}

Circulators based on non-reciprocal Faraday rotation effect have already presented before. In fact, Faraday rotation could also be applied to design phase shifters as illustrated in Figure 2.20 [9]. A linearly polarized wave entering the input port is converted to RHCP (right hand circular polarization) wave by the first $\lambda / 4$ plate, then in the ferrite region, the phase is delayed by $\beta_{+} z$, which can be adjusted by the bias field $H_{0}$. Finally the second $\lambda / 4$ plate converts RHCP wave back to a linear polarization. Similarly, a wave entering at the right, expects now a phase delay of $\beta_{-} z$ because of the non-reciprocity. The modes of wave propagation are: linear $T E_{10} \rightarrow$ circular $T E_{11} \rightarrow$ linear $T E_{10}$. However, if nonreciprocal quarter-wave plates are used, this phase shifter could be made reciprocal, because the linearly polarized wave is converted to the same sense of circular polarization for either propagation direction [9]. 


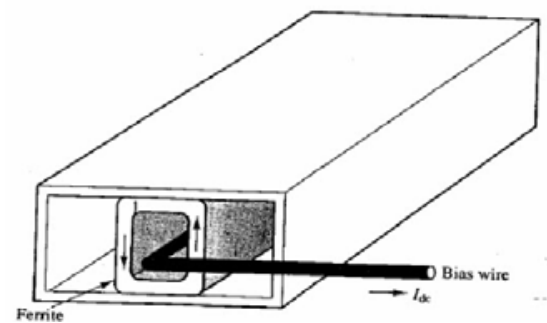

(a)

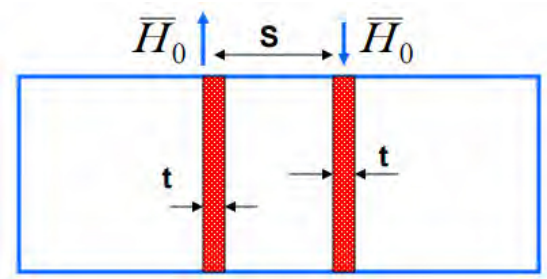

(b)

Figure 2.21: Geometry of a latched phase shifter (a) using a ferrite toroid (b) using 2 ferrite slabs [10]

\section{Nonreciprocal latching phase shifter}

When a ferrite toroid or two ferrite slabs are used in the waveguide as shown in Figure 2.21 [10], a latched phase shifter could also be realized. We magnetize the ferrite by a bias wire passing through the center of the ferrite. The rf signal is affected by the two sidewalls of toroid or the two ferrite slabs. The magnetic hysteresis of the ferrite is employed to create a phase shift which can be switched between two digital values. In practice, sections of ferrites have independent bias wires and lengths (cascade) leading to binary differential phase shifts of $45^{\circ}, 90^{\circ}, 180^{\circ}$ etc.

\section{Reggia-spencer reciprocal phase shifter}

In order to get the beam position for both transmission and reception, as required in scanning antenna phased arrays used in radar or communication systems, reciprocal phase shifters are desired. The commonly used reciprocal phase shifter is the reggia-spencer phase shifter as shown in Figure 2.22 [10]. The phase shifter usually uses rectangular or circulator waveguides. We place a ferrite rod in the waveguide and magnetize it longitudinally. The electromagnetic fields are bound to the ferrite forming a circular polarized wave under the condition that the diameter of the ferrite rod exceed a threshold value. Only a relative short length is needed to obtain a large 


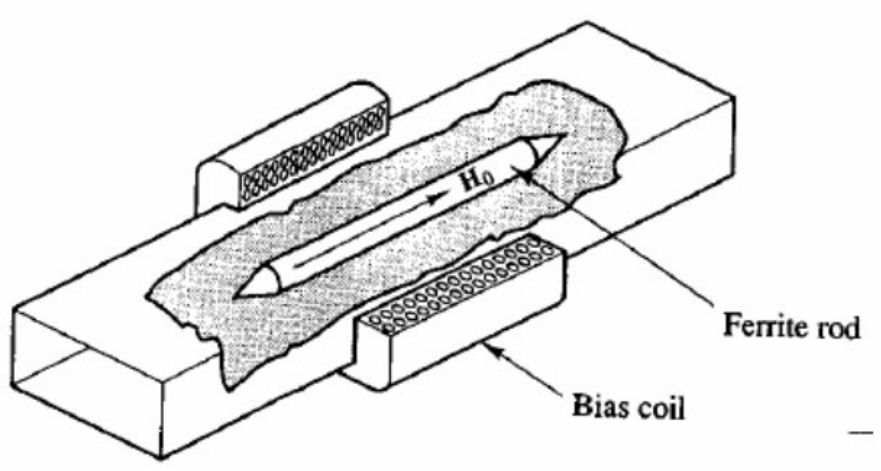

Figure 2.22: Reggia-Spencer phase shifter [10]

reciprocal phase shift. However, the phase shift is very sensitive to the operating frequency. The phase delay through the waveguide is proportional to the dc current through the coil, but independent of the direction of the propagation through the guide [9].

\subsection{Conclusion}

In this chapter, brief theories of microwave ferrite devices such as circulators, isolators, switches and phase shifters have been presented.

Circulators are mainly characterized by the insertion loss in the transmission direction, and the isolation in the opposite direction (blocking). The insertion loss should be as weak as possible $(<1 \mathrm{~dB})$ to avoid wasting energy, while the isolation is expected to be high $(>20 \mathrm{~dB})$ to reduce the interface between adjacent ports. Bandwidth of circulators could be chosen according to requirements. The typically fractional bandwidth is $10 \%$, or a specific range of frequency, like $\mathrm{X}$ band which is required in radar systems. Power capability is another important character which signifies the linearity of circulators. As is known, the temperature rise in the ferrite leads to the decreasing of the magnetization saturation $M_{s}$ which degrades the 
performance. The power capability varies from several single watts to mega-watts. Moreover, the circulator's frequency could be tuned by changing external magnetostatic field. Comparison of different types of circulators between coaxial, drop-in, surface mount, microstrip and waveguide are also presented. Compact structures like surface mount, and microstrip circulators have miniaturized size, while waveguide circulators typically support high power. This comparison shows a global image of different circulators at X-band in terms of the insertion loss, isolation, power and size.

There are different conceptions to design circulators varying from phase shift circulator to magnetic-free circulator as discussed previously. Each conception has its own advantages. Specific requirements are desired to determine the suitable design of the circulator. In order to support extreme high power, phase shift circulator is preferred. Faraday rotation circulators are often used as isolators. Ring circulators use couplers and phase shifters, however, ring circulators are barely used in practice. Junction circulators are well studies by researchers like H. Bosma and widely used in applications. In order to obtain a wide-band performance, we can use edge-guided circulators. Lumped-element circulators are used at low frequencies with small size. We employ self-biased circulators in applications operating at high frequencies (e.g. $>30 \mathrm{GHz})$.

Particularly, circulators based on LTCC technology are discussed. LTCC is a good candidate for $\mathrm{SiP}$ (system-in-package) system by integrating all active or passive devices into one substrate. This leads to a high integration and miniaturizes the rf front-end circuits. Conventional LTCC technology is not suitable to design ferrite devices because the traditional tape is non-magnetic. However, ferrite devices like circulators could be integrated into the LTCC substrate by co-firing ferrite tape and hence work with other rf components such as power amplifier, filters, mixers, antennas etc. 
There are various applications of circulators. The main role is to separate $T / R$ signals. Moreover, circulators could combine several transmitters with a shared antenna. Besides, by connecting a matched load to one of the circulator's ports, an isolator could be obtained.

Isolator is another important ferrite device. Three different conceptions are presented: resonance isolator, field displacement isolator and circulator based isolator. Resonance isolator is based on the gyromagnetic resonance of ferrite, while field displacement isolator relies on the difference of electric field distributions between the forward and reverse waves in a ferrite.

Finally, ferrite switches and phase shifter are discussed. Ferrite switches are mainly realized by controlling the external dc magnetic field. We can switch the ferrite's magnetization state by a current pulse through a wire in ferrite based on ferrite's magnetic hysteresis loop. In phased array antennas systems, we can steer the antenna's beam by electronically controlling the phase shifters. Phase shifter could be designed based on Faraday rotation, which uses the non-reciprocity of ferrites. Moreover, the reggiaspencer phase shifter is a reciprocal phase-shifter. Only a relatively short length is needed to obtain a large reciprocal phase shift. However, the phase shift is very sensitive to the operating frequency. 


\section{Chapter 3}

\section{Magnetic materials}

\subsection{Introduction}

Magnetic materials are used in many different applications and include a variety of categories. Power stations of electricity widely use magnetic materials [76]. Our storage media like computer disks and audio/video tapes also use magnetic materials as permanent magnets. In the field of medicine, we apply magnetic materials to body scanners. In our daily entertainment, magnetic materials are crucial for CD players, game consoles, loudspeakers etc. Fossil-fuel-free cars require high performance electric motors using advanced magnetic materials. Ferrimagnetic materials having high insulation are commonly used in microwave components such as circulators, phase shifters, isolators, tunable antennas, etc [32]. With the development of the telecommunications industry, high-speed data transmission and miniaturization of handsets are in demand requiring further improvement of magnetic materials [11]. Our modern society cannot work properly without magnetic materials, hence the study of magnetic materials is important to our world.

This chapter begins by outlining the various types of magnetic behaviors: diamag-

netism, paramagnetism, ferromagnetism, antiferromagnetism, and ferrimagnetism. 
Then different ferrimagnetic materials are presented. Moreover, the theories about ferrimagnetic materials are introduced, including the models of the permeability tensor for saturated ferrite and partially magnetized ferrite, demagnetization factor, loss, hysteresis loop, saturation magnetization, linewidth, etc. The control of the magnetic properties is one of the key aspects in designing magnets, recording media and microwave devices. Finally, ferrite Low Temperature Co-fired Ceramic (LTCC) materials are briefly discussed along with the conventional LTCC fabrication process.

\subsection{Classes of magnetic materials}

The ancient Egyptians, Greeks and Chinese firstly observed the magnetic phenomena and discovered the magnetic stone. Since then, human has been interested in magnetism. Many observations about the magnetic material like $\mathrm{Fe}_{3} \mathrm{O}_{4}$ were recorded by them [17].

According to the behavior of the orbital and spin motions under dc magnetic fields, the magnetic materials could be classified into different categories. These behaviors are quite different in various magnetic materials: in some materials, the interaction between the internal magnetic moments and the applied magnetic field is barely observed, while in some other materials, the interaction is firmly strong [32]. Consequently, five major categories of magnetic materials could be drawn: diamagnetic, paramagnetic, ferromagnetic, antiferromagnetic and ferrimagnetic. In other words, based on the phenomena, we can say diamagnetism, paramagnetism, ferromagnetism, antiferromagnetism, and ferrimagnetism [32]. 


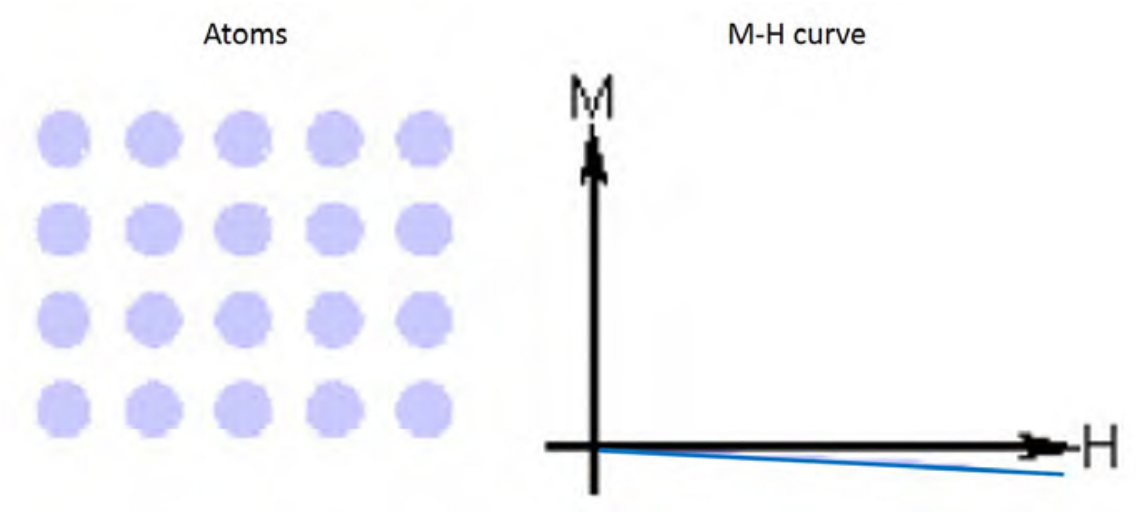

Figure 3.1: Atomic and magnetic behaviour of diamagnetism [11]

\subsubsection{Diamagnetism}

As in the case of covalent, the shells of the atoms in diamagnetism are closed solids. Diamagnetic materials include, for example, the semiconductors like $\mathrm{Ge}, \mathrm{Si}$, and metals such as $\mathrm{Au}, \mathrm{Cu}, \mathrm{Ag}$, etc. When the diamagnetic material is exposed under a dc magnetic field, a negative internal magnetization appears to fight against the external applied magnetic field [32]. The susceptibility, $\chi$, then, is negative (and weak) as shown in Figure 3.1 [11]. Diamagnetic substances contain the atoms without net magnetic moments. This is a fundamental property of this material because all the orbital shells are occupied leading to zero unpaired electrons. The M-H (M for magnetization and $\mathrm{H}$ for magnetic field) curve in Figure 3.1 shows that the magnetization $\mathrm{M}$ is in direct proportion to the applied magnetic field $\mathrm{H}$, but the slope is negative (susceptibility is both negative and small).

In addition, the susceptibility $\chi$ is temperature independent which is another main character [32]. Generally, diamgentic materials include all the non-magnetic materials like the semiconductors. However, when the semiconductors are doped with some rare earth elements such as Gd or transition metal elements such as Mn, they could be turned to be magnetic [32]. 


\subsubsection{Paramagnetism}

The diamagnetic materials, presented before, have the orbital shells which are filled and no unpaired electrons exist. However, paramagnetic materials possess atoms, molecules and lattice defects which lead to an unpaired number of electrons [32]. Hence, a non-zero total electron spin could appear.

There are some paramagnetic materials which could be attracted when an external dc magnetic field is applied because the induced internal magnetic fields appear and these fields have the same direction as the applied magnetic field [77]. Consequently, paramagnetic materials have a positive and small (usually greater than or equal to 1) susceptibility $\chi$ under the dc magnetic fields as shown in Figure 3.2 [11]. The magnetization $\mathrm{M}$ goes with the applied magnetic field $\mathrm{H}$ (see the $\mathrm{M}-\mathrm{H}$ curve) in paramagnetic materials. The slope of $\chi$ and the internal magnetization are both positive. When the external magnetic field is applied, paramagnetic materials could be slightly attracted, however, when the external magnetic field is removed, the magnetic properties disappear [32].

As explained, the presence of the unpaired electrons and the realignment of the electrons are the main reasons for the paramagnetic material's magnetic behavior [77]. According to the theory of the Langevin model about paramagnetism, the magnetic moment of each atom is randomly oriented because of the thermal agitations. A slight alignment of these moments is possible when a magnetic field is applied. Then a low magnetization having the same direction as the applied field could be obtained. The alignment of magnetization becomes more difficult when the temperature increases and hence the susceptibility becomes weak, as described by the Curie Law [77]. 

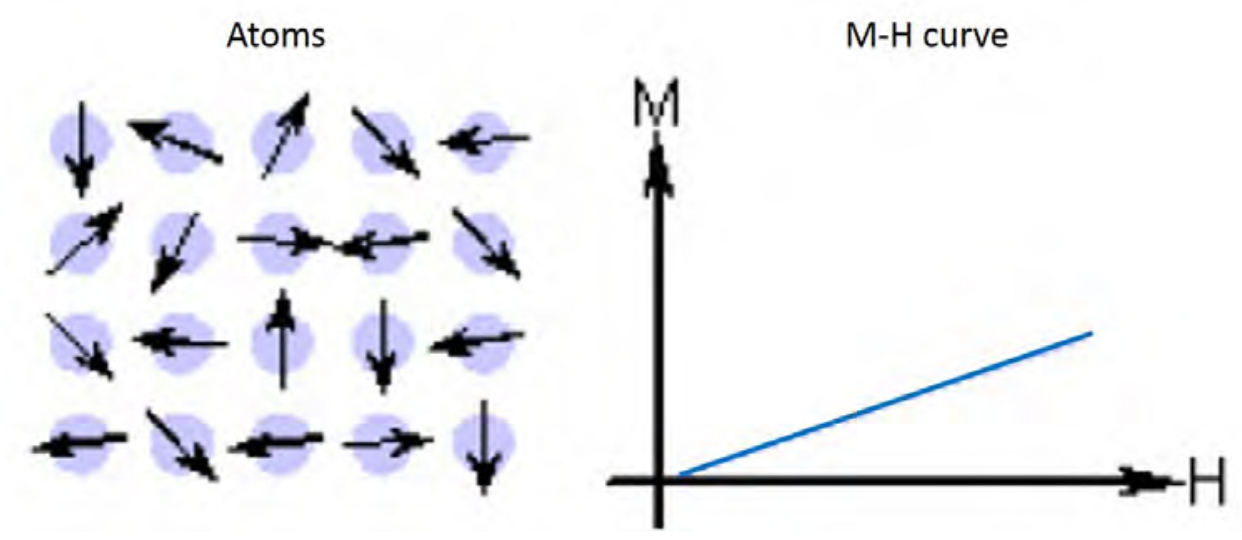

Figure 3.2: Atomic and magnetic behaviour of paramagnetism [11]

\subsubsection{Ferromagnetism}

Strictly, ferromagnetism is only possible when: 1 . the magnetic moments of all the atoms align parallel to each other; 2. atomic magnetic moments follow the applied magnetic field's direction; 3. all atoms are settled in a lattice as shown in Figure 3.3. The fact that all moments of individual atoms are aligned results in a large and positive susceptibility $\chi$ and a residual magnetization even when we remove the external magnetic field [32]. Ferromagnetism is basically used to form permanent magnets which can be formed by applying a strong external magnetic field. If we remove the external field, ferromagnetic materials still have the ability to keep the magnetization. Ferromagnetism is widely used in industry and modern technology, especially in a number of electrical and electromechanical devices such as generators, electric motors, electromagnets, transformers, magnetic storage like voice/video tape recorders, and $\mathrm{PC}$ hard disks, etc [78].

Initially, a classical theory, postulated first by Weiss in 1907, describes ferromagnetism. It is assumed that there is a molecular field in the ferromagnetic material [32]. Then, the Heisenberg model was proposed. It describes an exchange interaction of the parallel alignment of the magnetic moments between neighboring moments [32]. 
Atoms

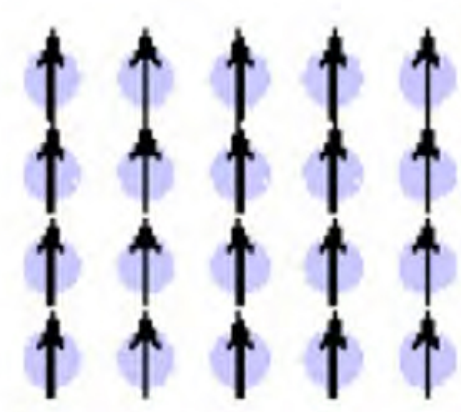

$\mathrm{M}-\mathrm{H}$ curve

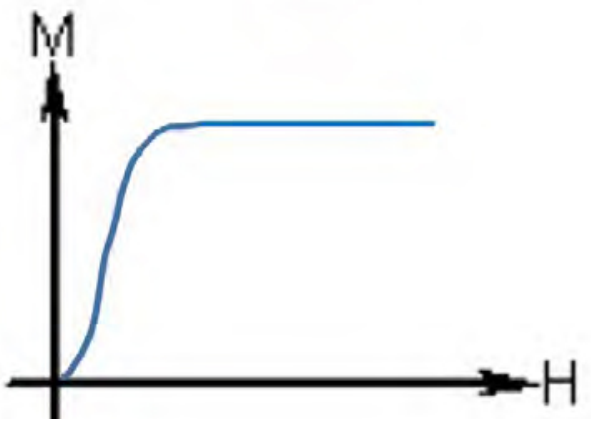

Figure 3.3: Atomic and magnetic behaviour of ferromagnetism [11]

When the temperature is lower than the Curie Temperature $T_{c}$, a hysteresis curve can be obtained showing the relation between the magnetization $\mathrm{M}$ and the magnetic field H. However, when the temperature goes beyond the Curie temperature, the thermal agitation becomes strong enough so that the materials turn to be paramagnetic. The Curie temperatures for some natural ferromagnetic elements are $T_{c}(\mathrm{Fe})=1043 \mathrm{~K}, T_{c}$ $(\mathrm{Co})=1404 \mathrm{~K}$ and $T_{c}(\mathrm{Ni})=631 \mathrm{~K}[32]$.

\subsubsection{Antiferromagnetism}

Atoms in antiferromagnetic materials have both parallel and anti-parallel aligned magnetic moments as shown in Figure 3.4. The magnetic moments' directions alternate from atom to atom with equal amplitude [79]. The net magnetization is not zero so that there is still a small slope $($ small $\chi)$ in $\mathrm{M}-\mathrm{H}$ curve. When the temperature is below Néel temperature, antiferromagnetism exists, however, the material turns to be paramagnetic when temperature is at or above the Néel temperature [80]. 
Atoms

$\mathrm{M}-\mathrm{H}$ curve
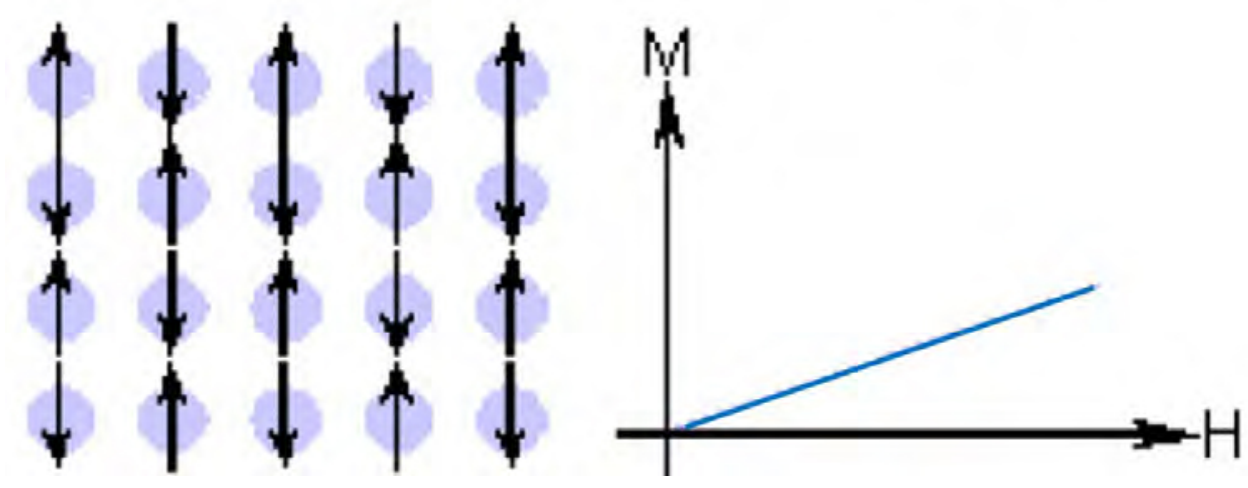

Figure 3.4: Atomic and magnetic behaviour of antiferromagnetism [11]

\subsubsection{Ferrimagnetism}

Similar to ferromagnetism, ferrimagnetic materials have a positive and large susceptibility which is a function of the applied field $H_{a}$. However, the atoms have unequal (amplitude) anti-parallel aligned magnetic moments leading to a magnetization saturation $M_{s}$ [79]. Even at zero Kelvin, not all the moments in ferrimagnetic materials align parallel [32].

The main advantage of ferrimagnetic materials is that they are non-conducting, hence they are widely employed in radio frequency applications as substrates. Since ferrimagnetic material is used in microwave devices as substrates, it is also called microwave ferrite and the next section will introduce more about it.

\subsection{Ferrite materials}

\subsubsection{Introduction}

Ferrite materials are used at microwave frequencies, with a high resistivity (not conductors), a low dielectric loss $\left(\tan \delta \approx 10^{-3}\right.$ or $\left.10^{-4}\right)$ and a dielectric constant between 


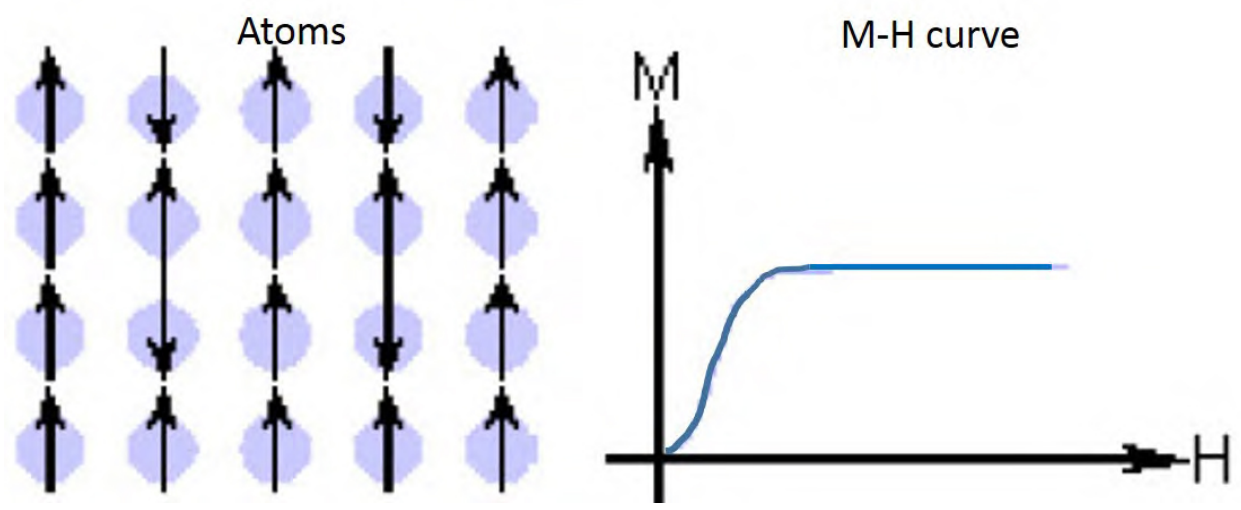

Figure 3.5: Atomic and magnetic behaviour of ferrimagnetism [11]

11 and 17. They are divided into four main categories according to their crystallographic structures [32].

The first is that of spinel ferrites, crystallizing in a face-centered cubic lattice. These are soft ferrites used in inductors, transformers, circulators etc.

The second family concerns the garnets. Their applications lie in the frequency range 0.1 - $10 \mathrm{GHz}$. YIG (Yttrium-Iron Garnet) is a well known garnet ferrite possessing a magnetic saturation $\mu_{0} M_{s}$ around $0.18 \mathrm{~T}$. Other garnet ferrites, like Y-Al (Yttrium Aluminum) ferrites which have a narrow linewidth (magnetic loss), are utilized to design low-loss devices. Moreover, Dy-Doped (Dysprosium-doped) power ferrite materials are employed for high peak power level applications because of the good temperature stability.

The third family is the hexagonal ferrites, or called hexaferrites, such as barium hexaferrite $\mathrm{BaFe}_{12} \mathrm{O}_{19}$. These ferrites are used in devices with high frequencies and have a high internal magneto-crystalline anisotropy field. 


\subsubsection{Different types of ferrite}

\section{Spinel}

The spinel ferrite has a structure $\mathrm{MeFe}_{2} \mathrm{O}_{4}$, where Me represents the metal. Spinel structure materials are closed packed cubic where $M e^{2+}$ and $M e^{3+}$ are at two different crystallographic sites [79]. Ferrites with spinel crystal structure are mainly used in the frequency range 3-30 $\mathrm{GHz}[46]$.

Spinels are among the most important mineral oxides and a source of metals in the extractive industry. The lodestone or magnet, is the most abundant natural magnetic spinel. Spinel ferrites can be considered as derivatives of magnetite by adding the atoms of other elements like $\mathrm{Ni}, \mathrm{Mn}, \mathrm{Zn}, \mathrm{Mg}$, Li etc. The magnetic properties of spinel ferrites such as permeability, are sensitive to the chemical composition.

There are two principle types of spinel ferrites [14]: 1. Mn-Mg (ManganeseMagnesium) ferrites which are used in devices requiring low magnetic and dielectric losses; 2. Li (or Titanium, Zinc) ferrites which are usually used in the production of temperature-stable components operating at or above X-band.

\section{Garnet}

Ferrites with garnet structure are made up of rare-earth elements $R^{3+}$, such as $G d^{3+}$ (Gadolinium), $T b^{3+}$ (Terbium), $Y^{3+}$ (Yttrium) etc and have the general formula $\mathrm{R}_{3} \mathrm{Fe}_{5} \mathrm{O}_{12}$. The garnet structure is cubic and the most common form is the iron garnet and yttrium $\mathrm{Y}_{3} \mathrm{Fe}_{5} \mathrm{O}_{12}$, known as YIG (Yttrium Iron Garnet). Garnets have a low saturation magnetization $M_{s}$ and low Curie temperature, limiting their use in the frequency range $10 \mathrm{MHz}$ to $10 \mathrm{GHz}$. They also exhibit low magnetic losses $(\Delta H$ and $\left.\Delta H_{e f f}\right)$. This type of ferrites is usually used in applications such as filters and oscillators, as well as in microwave non-reciprocal devices like the YIG resonator and 
circulators.

\section{Hexaferrite}

The common known structure of hexaferrite is the M-type with the stoichiometric chemical formula $\mathrm{BaFe}_{12} \mathrm{O}_{19}$. M-type structure has a feature which is "hard", meaning a strong axial magnetic anisotropy up to $2800 \mathrm{kA} / \mathrm{m}$, equivalent to 100 to 1000 times that of spinels or garnets. In practice, permanent magnets are commonly based on the M-type hexagonal barium hexaferrite or BaM. Another main application of this material is the high density magnetic recording medias [81]. Other structures like Z-type, W-type or Y-type, have a planar anisotropy which is "soft", allowing a high permeability in the range of microwave.

The high value of anisotropy in M-type hexaferrite allows the design of millimeter devices in the frequency range from $30 \mathrm{GHz}$ to about $100 \mathrm{GHz}$. This makes them attractive for the realization of miniaturized devices which do not require huge external polarizations (operated as self-biased). These materials have a saturation magnetization around $400 \mathrm{kA} / \mathrm{m}\left(\mu_{0} M_{s}=0.5 T\right)$, a linewidth greater than $4 \mathrm{kA} / \mathrm{m}$ and a Curie temperature about $450{ }^{\circ} \mathrm{C}[46]$.

\subsection{Basic properties of ferrite materials}

\subsubsection{Models of permeability tensor}

As is known, magnetic materials may be anisotropic, in which case a permeability tensor instead of a constant should be used. This section presents different theories to model permeability tensors for saturated ferrites and partially magnetized ferrites. 


\section{Polder (Saturated model)}

The existence of magnetic dipole moments leads to the magnetic properties of the ferrite. The magnetic dipole moments come primarily from the electron spin. The Landé $g$ factor is a constant to measure the relative contributions of the orbital moment and the spin moment to the total magnetic moment. In practice, $\mathrm{g}=2$ is a good approximation for most microwave ferrite materials which signifies that the moment is mostly due to the electron spin. The magnetic dipole moment of an electron due to its spin is given by Equation (3.1) [9].

$$
m=\frac{q \hbar}{2 m_{e}}=9.27 \times 10^{-24} A \cdot m^{2}
$$

where $\hbar$ is Planck's constant divided by $2 \pi$, q is the electron charge, and $m_{e}$ is the mass of the electron [9]. Moreover, the spin angular momentum of an electron is given by Equation (3.2).

$$
s=\frac{\hbar}{2}
$$

Another important constant is gyromagnetic ratio $\gamma$ which is the ratio of the spin magnetic moment to the spin angular momentum as expressed by Equation (3.3). In addition, these two vectors are oppositely directed as shown in Figure 3.6.

$$
\gamma=\frac{m}{s}=1.759 \times 10^{11} \mathrm{C} / \mathrm{Kg}=2.8 \mathrm{MHz} / \mathrm{Oe}
$$

If the ferrite medium is infinite and a magnetic bias field $\vec{H}_{0}=\vec{z} H_{0}$ is present, the motion of the magnetic dipole moment could be derived (see Equation (3.4)) [9].

$$
\frac{d \vec{m}}{d t}=-\mu_{0} \gamma \vec{m} \times \vec{H}_{0}
$$




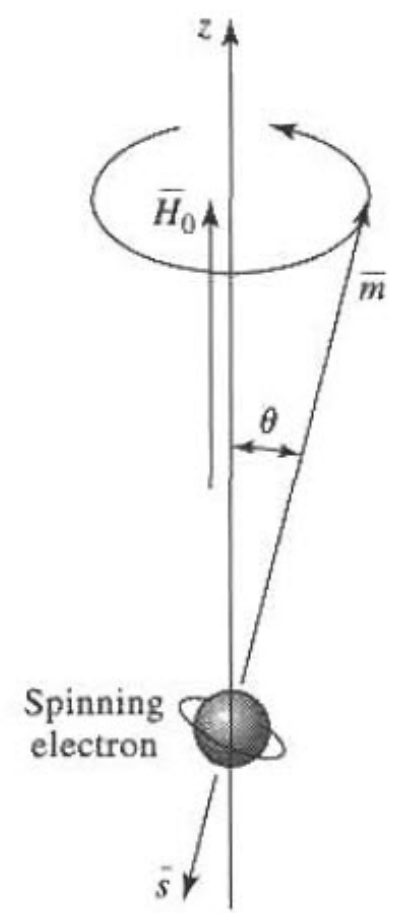

Figure 3.6: A spining electron: Spin magnetic dipole moment $(\vec{m})$ and angular momentum vectors $(\vec{s})[9]$

Moreover, if an applied AC field $\vec{H}$ is present and the magnetization M reaches the saturation $M_{s}$, then the equations of motion for the forced precession of the magnetic dipoles, assuming small-signal conditions, could be expressed by Equation (3.5), where $\omega_{0}=\mu_{0} \gamma H_{0}$ and $\omega_{m}=\mu_{0} \gamma M_{s}$.

$$
\begin{aligned}
& \frac{d^{2} M_{x}}{d t^{2}}+\omega_{0}^{2} M_{x}=\omega_{m} \frac{d H_{y}}{d t}+\omega_{0} \omega_{m} H_{x} \\
& \frac{d^{2} M_{y}}{d t^{2}}+\omega_{0}^{2} M_{y}=\omega_{m} \frac{d H_{x}}{d t}+\omega_{0} \omega_{m} H_{y}
\end{aligned}
$$

Besides, when the AC $\vec{H}$ field has an $e^{j \omega t}$ time-harmonic dependence, these equations could be re-written with a tensor susceptibility, $[\chi]$ to relate $\vec{H}$ and $\vec{M}$ as shown in Equation (3.6). 


$$
\vec{M}=[\chi] \vec{H}=\left[\begin{array}{ccc}
\chi_{x x} & \chi_{x y} & 0 \\
\chi_{y x} & \chi_{y y} & 0 \\
0 & 0 & 0
\end{array}\right] \vec{H}
$$

The elements of $\chi$ are given by Equation (3.7):

$$
\begin{gathered}
\chi_{x x}=\chi_{y y}=\frac{\omega_{0} \omega_{m}}{\omega_{0}^{2}-\omega^{2}} \\
\chi_{x y}=-\chi_{y x}=\frac{j \omega \omega_{m}}{\omega_{0}^{2}-\omega^{2}}
\end{gathered}
$$

Hence Equation (3.8) relates $\vec{B}$ and $\vec{H}$ by the permeability tensor when the magnetic bias is in the $\vec{z}$ direction, where $\mu_{+}$and $\mu_{-}$is the effective permeability for RHCP (Right Hand Circular Polarization) wave and LHCP (Light Hand Circular Polarization) wave, respectively.

$$
\begin{gathered}
\vec{B}=\mu_{0}(\vec{M}+\vec{H})=[\mu] \vec{H} \\
{[\mu]=\mu_{0}([U]+[\chi])=\left[\begin{array}{ccc}
\mu & j \kappa & 0 \\
-j \kappa & \mu & 0 \\
0 & 0 & \mu_{0}
\end{array}\right] \Leftarrow(\vec{z}-\text { bias })} \\
\mu=\mu_{0}\left(1+\frac{\omega_{0} \omega_{m}}{\omega_{0}^{2}-\omega^{2}}\right) \\
\kappa=\mu_{0} \frac{\omega \omega_{m}}{\omega_{0}^{2}-\omega^{2}} \\
\mu_{+}=\mu+\kappa
\end{gathered}
$$




$$
\mu_{-}=\mu-\kappa
$$

If the ferrite is biased in other directions, the permeability tensor could be transformed according to the change in coordinates as demonstrated by Equation (3.9a) [82] where $\alpha, \beta$ and $\gamma$ is the angle against the axis $\mathrm{x}, \mathrm{y}$ and $\mathrm{z}$ respectively. When $H_{0}$ is parallel to the axis $\mathrm{x}$, it is easy to find $\cos \alpha=1$ and $\cos \beta=\cos \gamma=0$ leading to Equation (3.9b). When $H_{0}$ is parallel to the axis y, then $\cos \beta=1$ and $\cos \alpha=\cos \gamma=0$ resulting in Equation (3.9c).

$$
\begin{gathered}
{[\mu]=\left[\begin{array}{ccc}
\mu\left(\mu_{0} \text { when } \vec{x} \text { biased }\right) & j \kappa \cos \gamma & -j \kappa \cos \beta \\
-j \kappa \cos \gamma & \mu\left(\mu_{0} \text { when } \vec{y} \text { biased }\right) & -j \kappa \cos \alpha \\
j \kappa \cos \beta & j \kappa \cos \alpha & \mu\left(\mu_{0} \text { when } \vec{z} \text { biased }\right)
\end{array}\right]} \\
{[\mu]=\left[\begin{array}{ccc}
\mu_{0} & 0 & 0 \\
0 & \mu & -j \kappa \\
& & \\
0 & -j \kappa & \mu
\end{array}\right] \Leftarrow(\vec{x}-\text { bias })} \\
{[\mu]=\left[\begin{array}{ccc}
\mu & 0 & -j \kappa \\
0 & \mu_{0} & 0 \\
j \kappa & 0 & \mu
\end{array}\right] \Leftarrow(\vec{y}-\text { bias })}
\end{gathered}
$$

Figure 3.7 [82] shows the geometrical relations between $\mu_{+}, \mu_{-}, \mu, \kappa, \mu_{\text {eff }}$ and 


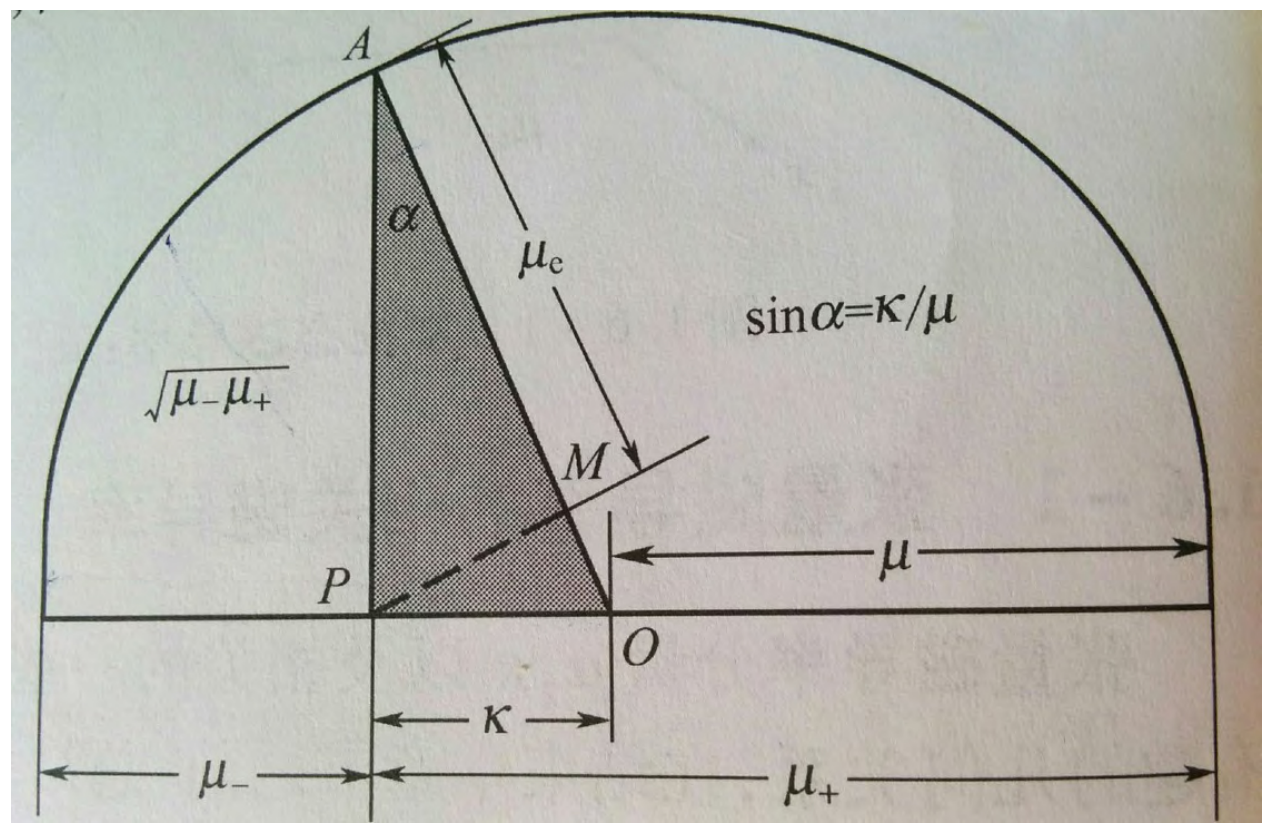

Figure 3.7: Geometric relation of different elements of permeability tensor

$\mu_{+} \mu_{-}=\mu^{2}-\kappa^{2}$ according to Equations (3.8e) and (3.8f). The non-reciprocity increases with the increase of $\sin \alpha=\frac{\kappa}{\mu}$. Noting that when $\kappa=0$ then $\sin \alpha=\frac{\kappa}{\mu}=0$ leading to the disappearance of non-reciprocity.

The above is the well-known Polder model. Since ferrites are commonly used under saturated state, this model is predefined in many EM simulators to perform ferritebased simulations. However, partially magnetized ferrites are also used, because a small internal magnetic $\mathrm{H}$ field could easily vary the internal magnetization $\mathrm{M}$ which is useful for miniaturized tunable devices [27]. Next parts will present the theories about demagnetized ferrites and partially magnetized ferrites.

\section{Schlömann (Demagnetized Model)}

Enlightened by a study of coaxial configuration where parallel and antiparallel domains are alternatively magnetized, Schlömann [83] developed his theory based on 
a magnetostatic approximation. This theory takes into consideration the interactions between domains of opposite magnetization [84]. When the ferrite is completely demagnetized, the diagonal values of the permeability tensor have an accurate approximation for the isotropic permeability [84]. Schlömann shows that the permeability $\mu_{r}$ takes the following form as shown by Equation (3.10a), where $\mu_{\text {eff }}$ is defied in Equation (3.10b) and the $H_{a}$ is the anisotropy field [83].

$$
\begin{aligned}
{\left[\mu_{r}\right] } & =\left[\begin{array}{ccc}
\mu_{e f f} & 0 & 0 \\
0 & \mu_{e f f} & 0 \\
0 & 0 & \mu_{e f f}
\end{array}\right] \\
\mu_{e f f}=\frac{1}{3}+\frac{2}{3} & {\left[\frac{\left(H_{a}+4 \pi M_{0}\right)^{2}-(\omega / \gamma)^{2}}{H_{a}^{2}-(\omega / \gamma)^{2}}\right]^{0.5} }
\end{aligned}
$$

Based on the assumptions of E. Schlömann, the internal field is identified with the anisotropy field of the material as the applied field is completely offset by the static demagnetizing fields. This model is only valid when a ferrimagnetic material is totally demagnetized.

\section{Rado (Partially Magnetized Model)}

Another theory, proposed by Rado [85], saying that we perform a spatial average of the responses produced by all domains in the ferrite. When the frequencies exceed the gyroresonance frequency, Rado's theory offers an accurate value for the extra-diagonal term $\kappa$, but the diagonal ones $\left(\mu=\mu_{z}=1\right)$ are not accurate [84]. Unfortunately, this theory does not take into consideration the interactions between adjacent areas. The results obtained by Rado are expressed by Equations (3.11a) and (3.11b), where $\omega_{m}=\mu_{0} \gamma M_{s}$. It is assumed that the working frequency is much higher than the 
resonant frequencies $\omega \gg \omega_{0}[46]$.

$$
\begin{gathered}
\mu=\mu_{z}=1 \\
\kappa=-\frac{\omega_{m}}{\omega} \frac{M}{M_{s}}
\end{gathered}
$$

\section{Igarashi and Naïto (Partially Magnetized Model)}

Igarashi and Naito [86] presented a formula for the transverse diagonal term in a partially magnetized state ferrite. This model is a further improvement of the tensor model of Schlömann. In the publication [87], the expressions of the tensor terms are given by Equations (3.12a) to (3.12d), where $\alpha$ is a phenomenological loss term, $\omega_{M}=-\gamma M_{s} / \omega_{0}, \gamma$ is the gyromagnetic ratio, $\mu_{0}$ is the intrinsic permeability of free space, and $\omega_{e}$ is a parameter concerned with the effective dc field. However, we need to perform some preliminary measurements to determine the $\omega_{e}$, especially when the magnetic field $\mathrm{H}$ is relatively high.

$$
\begin{gathered}
\mu_{z}=1+(\mu-1)\left[1-\left(\frac{M}{M_{s}}\right)^{2}\right] \\
\mu=1+\frac{\omega_{m}\left(\omega_{e}+j \alpha \omega\right)}{\left(\omega_{e}+j \alpha \omega\right)^{2}-\omega^{2}} \\
\kappa=-\frac{\omega_{m} \omega}{\left(\omega_{e}+j \alpha \omega\right)^{2}-\omega^{2}} \\
\omega_{e}=\gamma H_{a}
\end{gathered}
$$




\section{Gelin GPT (Generalized Permeability Model)}

Previous theories use actually the average responses of each domain. For example Schlömann developed a model "magnetostatic" by taking into account the interaction domains with antiparallel magnetization. In the case where the ferrite is demagnetized, the model is in good agreement with the experiment. However, Schlömann cannot process the case of partially magnetized ferrites.

To describe the permeability of the ferrites (saturated, partially magnetized or demagnetized) correctly, a theoretical approach was developed [84,88-90]. Mathematically, this approach means solving two coupled evolution following Equations (3.13a) and $(3.13 \mathrm{~b})$, where $\mathrm{n}$ is a demagnetizing factor which depends on the shape of the ferrite, $\mathrm{h}$ are the microwave magnetic field, $H_{1}$ and $H_{2}, M_{1}$ and $M_{2}, m_{1}$ and $m_{2}$ is the static magnetic field, macroscopic magnetization, and the magnetization dynamics induced by $\vec{h}$ in the domain 1 and domain 2 , respectively.

$$
\begin{aligned}
& \frac{d \vec{M}_{1}}{d t}=\gamma \vec{M}_{1} \times\left(\vec{H}_{1}+\vec{h}-n\left(\vec{m}_{1}-\vec{m}_{2}\right)\right)+\frac{\alpha}{M_{s}} \vec{M}_{1} \times \frac{d \vec{M}_{1}}{d t} \\
& \frac{d \vec{M}_{2}}{d t}=\gamma \vec{M}_{2} \times\left(\vec{H}_{2}+\vec{h}-n\left(\vec{m}_{2}-\vec{m}_{1}\right)\right)+\frac{\alpha}{M_{s}} \vec{M}_{2} \times \frac{d \vec{M}_{2}}{d t}
\end{aligned}
$$

The permeability tensor based on the Equations (3.13a) and (3.13b) allows a more rigorous description of the interaction between the rf signal and the magnetic moments in ferrites. This model also considers the dynamic interactions between adjacent magnetic fields via the Polder-Smit effect when these domains appear in the material. The interactions between the grains and the hysteresis phenomenon are also considered. By altering the dynamic demagnetizing effects between neighboring domains of the ferrite, and therefore by changing the distribution of the values of 
the frequencies of gyromagnetic resonance, the Polder-Smit effect induces the spreading of magnetic losses in relation to the frequency. This has in fact been observed experimentally in unsaturated materials [46].

This model is valid for saturated, partially magnetized and demagnetized ferrites. It is accurate, however, it is difficult to be implemented into EM simulator because of the integral equations.

\section{Green and Sandy (Partially Magnetized Model)}

In this thesis, we use Green and Sandy's model. Based on the measurements of the experimental characterization cells, Green and Sandy [91] have obtained all the tensor values when ferrite is totally demagnetized. They developed the diagonal terms $(\mu$ and $\mu_{z}$ ) which are compatible with Schlömanns formula for a totally demagnetized ferrite [84].

The equations for the tensor elements of a partially magnetized ferrite material when using Z-bias are given by Equations (3.14a) to (3.14f), where $\mu_{0}^{\prime}$ is the initial relative permeability of the substrate in the demagnetized state, $\omega$ is the device's operating frequency, the relative $\mu$ and $\kappa$ values replace the standard elements of the Polder permeability tensor, $\mathrm{M}$ and $M_{s}$ are the partial and saturation magnetization, respectively and $\mathrm{n}$ is the coefficient expressing the deviation of $\kappa$ from Rado's mode.

$$
\begin{gathered}
\text { Permeability }=\mu_{0}\left[\begin{array}{ccc}
\mu & -j \kappa & 0 \\
j \kappa & \mu & 0 \\
0 & 0 & \mu_{z}
\end{array}\right] \\
\mu_{0}^{\prime}=\frac{2}{3}\left[1-\left(\frac{\gamma 4 \pi M_{s}}{w}\right)^{2}\right]^{0.5}+\frac{1}{3}
\end{gathered}
$$




$$
\begin{gathered}
\mu=\mu_{0}^{\prime}+\left(1-\mu_{0}^{\prime}\right)\left(\frac{M}{M_{s}}\right)^{1.5} \\
\kappa=n \frac{\gamma 4 \pi M}{w} \\
\mu_{z}=\mu_{0}^{\prime}\left(1-\left(\frac{M}{M_{s}}\right)^{2.5}\right) \\
\mu_{\text {eff }}=\frac{\mu^{2}-\kappa^{2}}{\mu}
\end{gathered}
$$

The model is valid when the operating frequency is higher than the resonance, and it has been used to analyze an antenna based on a partially magnetized ferrite LTCC substrate ESL 40012 and has proven to be a good approximate model [28].

Therefore, we use Green and Sandy's model in this thesis because it is valid for our design and relatively easy to be used in our simulators. Moreover, it has been employed on ferrite LTCC ESL 40012 substrate which is also used in this thesis.

\subsubsection{Demagnetization factors}

When the ferrite is not infinite, the internal bias field $H_{0}$ is generally different from the externally applied field $H_{a}$. According to the boundary conditions at the surface of the ferrite, the magnetic flux $\vec{B}$ is continuous when the applied field is perpendicular to the ferrite plate. However, the magnetic field $\mathrm{H}$ is not continuous. Equation (3.15) demonstrates that the internal magnetic bias field is $H_{0}=H_{a}-M_{s}$. Of course, when

applied field is parallel to the ferrite plate, then the magnetic field $\vec{H}$ is continuous leading to $H_{0}=H_{a}$.

$$
B=\mu_{0} H_{a}=\mu_{0}\left(M_{s}+H_{0}\right)
$$



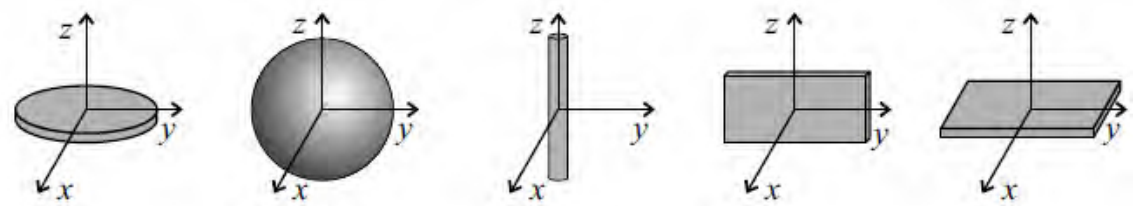

$\begin{array}{ll}N_{x} & 0 \\ N_{y} & 0 \\ N_{z} & 1\end{array}$

$1 / 3$
$1 / 3$
$1 / 3$

$1 / 2$

$1 / 2$

0
1

0

0
0

0

1

Figure 3.8: Demagnetization factors of some simple shapes [12]

Generally, the demagnetization factor $(\mathrm{N})$ is used to measure the deduction of the applied field as is shown by Equation (3.16), where $\mathrm{N}=N_{x}, N_{y}$ or $N_{z}$. The demagnetization factors are defined such that $N_{x}+N_{y}+N_{z}=1$. A few simple shapes' demagnetization factors are listed in Figure 3.8 [12].

$$
\vec{H}_{i}=\vec{H}_{a}-N \vec{M}
$$

The demagnetization factor is also related to the internal and external rf fields near the boundary of a ferrite sample. This leads to the Kittel's equation which is used to calculate the resonance frequency $\omega_{r}$ as shown by Equation (3.17), where $H_{a}$ is the applied magnetic field [92].

$$
\omega_{r}=\mu_{0} \gamma \sqrt{\left[H_{a}+\left(N_{x}-N_{z}\right) M_{s}\right]\left[H_{a}+\left(N_{y}-N_{z}\right) M_{s}\right]}
$$

\subsubsection{Effect of loss}

If the damping factor $\alpha$ is also considered, the Polder tensor elements are given by Equations (3.18) and (3.19a) to (3.19d) [9]: 


$$
\begin{gathered}
\chi_{x x}=\chi_{x x}^{\prime}-j \chi_{x x}^{\prime \prime} \\
\chi_{x y}=\chi_{x y}^{\prime \prime}+j \chi_{x y}^{\prime} \\
\chi_{x x}^{\prime}=\frac{\omega_{0} \omega_{m}\left(\omega_{0}^{2}-\omega^{2}\left(1-\alpha^{2}\right)\right)}{\left(\omega_{0}^{2}-\omega^{2}\left(1+\alpha^{2}\right)\right)^{2}+4 \alpha^{2} \omega^{2} \omega_{0}^{2}} \\
\chi_{x x}^{\prime \prime}=\frac{\alpha \omega \omega_{m}\left(\omega_{0}^{2}+\omega^{2}\left(1+\alpha^{2}\right)\right)}{\left(\omega_{0}^{2}-\omega^{2}\left(1+\alpha^{2}\right)\right)^{2}+4 \alpha^{2} \omega^{2} \omega_{0}^{2}} \\
\chi_{x y}^{\prime}=\frac{\omega \omega_{m}\left(\omega_{0}^{2}-\omega^{2}\left(1+\alpha^{2}\right)\right)}{\left(\omega_{0}^{2}-\omega^{2}\left(1+\alpha^{2}\right)\right)^{2}+4 \alpha^{2} \omega^{2} \omega_{0}^{2}} \\
\chi_{x y}^{\prime \prime}=\frac{2 \alpha \omega^{2} \omega_{0} \omega_{m}}{\left(\omega_{0}^{2}-\omega^{2}\left(1+\alpha^{2}\right)\right)^{2}+4 \alpha^{2} \omega^{2} \omega_{0}^{2}}
\end{gathered}
$$

Then the lossy tensor $[\mu]$ can be calculated using Equation (3.8b). The damping factor $\alpha$ has a relation with the linewidth of ferrite materials which is shown by Equation (3.20) [9]. Ferrite materials with higher linewidth $\Delta H$ have more magnetic losses. Noting that this loss is separate from the dielectric loss that a ferrimagnetic material may have at the same time.

$$
\Delta H=\frac{2 \alpha \omega}{\mu_{0} \gamma}
$$

\subsubsection{Hysteresis loop}

Figure 3.9 [13] shows an example of a magnetic hysteresis loop regarding the induced magnetic flux density (B) in terms of the magnetic field $(\mathrm{H})$. The hysteresis loop of a ferromagnetic material could be obtained by tracing the function of internal magnetic 


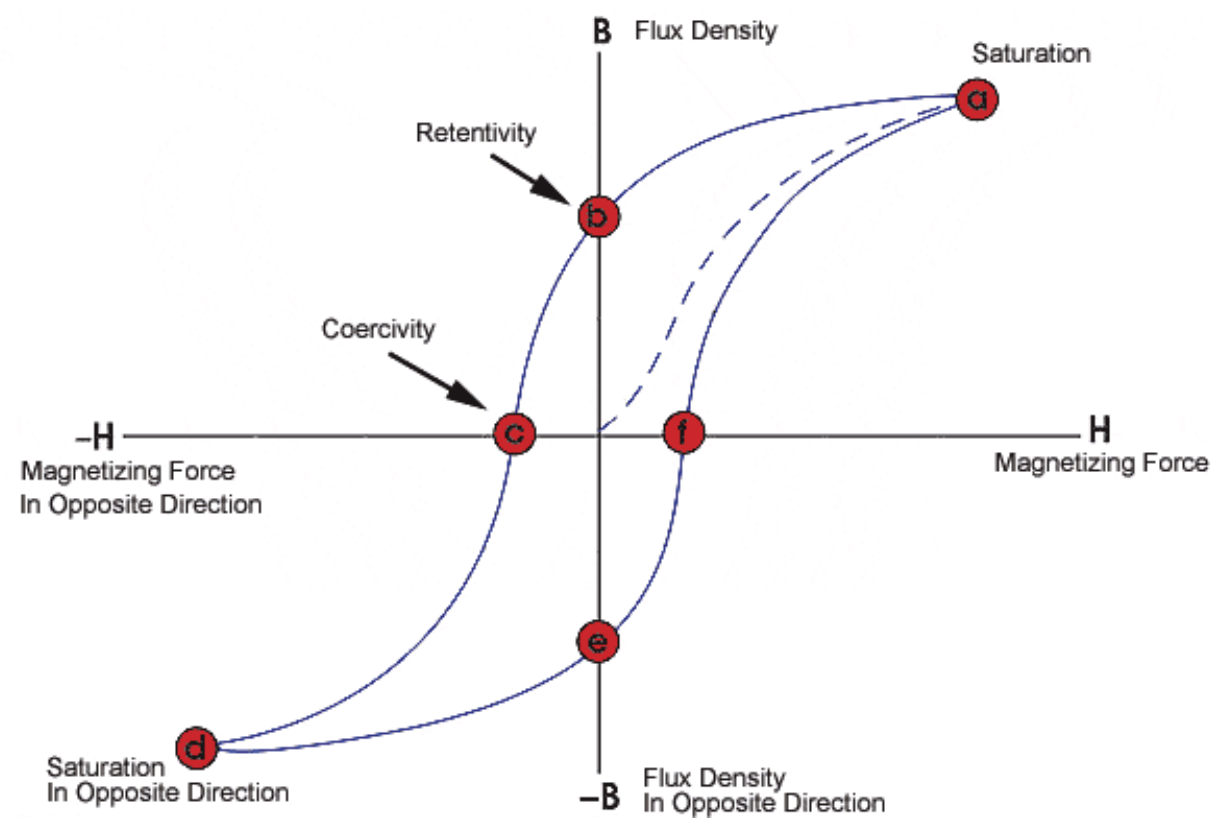

Figure 3.9: An example of magnetic hysteresis loop [13]

flux B as the applied magnetic field H. It's important to note that when the ferromagnetic material is the first time to be magnetized or it has been totally demagnetized, magnetic flux density B will follow the dashed line, also called the initial line, as shown in Figure 3.9. Magnetic field flux B is proportional to $\mathrm{H}$, however, when all the magnetic moments in the ferromagnetic material are aligned, the increase of $\mathrm{H}$ will hardly contribute to the increase of $\mathrm{B}$, hence the material reaches the saturation point (point a). Now, if we remove the applied $\mathrm{H}$, the magnetic flux will drop to point b, meaning that there are still some aligned magnetic moments. This refers to the retentivity of the ferromagnetic material. The presence of retentivity is a key property for ferromagnetic, and is widely used to produce permanent magnets. It is worthwhile to note that when the magnetic field $\mathrm{H}$ is reversed, $\mathrm{B}$ will move to point $\mathrm{c}$, also called the point of coercivity, which suggests that the magnetic flux is reset-off to zero (demagnetized). The magnetic field $\mathrm{H}$ required to release the residual magnetism of the ferromagnetic material is called coercivity [13]. 
Similarly, the ferromagnetic material could have the magnetic saturation when $\mathrm{H}$ is increased in the opposite direction. This time the saturation is the point $\mathrm{d}$ as shown in the figure. The release of $\mathrm{H}$ will move the curve to point e. The residual magnetism is the same as obtained in the opposite direction. In this case, a positive $\mathrm{H}$ should be used to bring $\mathrm{B}$ back to zero. It is noteworthy that the curve will follow the line from point $\mathrm{f}$ to point a instead of the initial dash line if $\mathrm{H}$ continues increasing [13].

Some important magnetic properties could be obtained based on the hysteresis loop:

Retentivity: The remaining internal magnetization (the point $\mathrm{b}$ in the hysteresis curve) of a magnetic material when the external dc magnetic field $\mathrm{H}$ is removed.

Residual Magnetism or Residual Flux: The residual magnetism equals to retentivity when the material reaches the magnetic saturation and then the applied magnetic field $\mathrm{H}$ is released. However, if the material does not reach the saturation, the residual magnetism is smaller than the retentivity.

Coercive Force: The magnetic field needed to bring the magnetic flux density B back to zero (the value of $\mathrm{H}$ at point $\mathrm{c}$ )

Maximum Energy Product (BHmax): It measures the maximum energy product of a magnetic material, which uses the unit Mega Gauss Oersteds (MGOe)

Squareness: It is the result of the saturation remanence (residual magnetization) divided by the saturation magnetization.

Material Grade: The manufactured neodymium (NdFeB) magnets are classified into different grades. Generally, the stronger magnet has higher grade. The typical range of grades is N35 to N52 for neodymium magnets. The limit for neodymium magnets is the grade N64 in theory, meaning that it isn't currently possible to realize the magnets such strong. The grade of commercialized magnets is usually N42 because N42 provides the optimal trade-off between strength and cost. 


\subsubsection{Magnetization $M_{s}$}

As explained before, with the increase of the applied field, more magnetic dipoles are

aligned, until all are aligned. In this case, $\vec{M}$ reaches its upper limit called magnetic saturation $M_{s}$. Since the ferrite material is a soft magnetic material, a small field (close to coercive force $H_{c}$ ) of about 1 to few Oe is enough to get the magnetization value $\mathrm{M}$ close to its saturated maximum $M_{s}$ (values of $\mu_{0} M_{s}$ are in the range $29 \mathrm{mT}$ to $500 \mathrm{mT})$.

\subsubsection{Curie Temperature $T_{c}$}

By increasing the temperature of the ferrite, the aligning of the spins parallel to the $\mathrm{H}$ field is more and more difficult due to the thermal agitation. The magnetization becomes null at the Curie Temperature $T_{c}$, which is in the range of $120^{\circ} \mathrm{C}$ to $650^{\circ} \mathrm{C}$ [14].

\subsubsection{Linewidth $\Delta H$}

Figure 3.10 [9] shows the definition of the linewidth: the width of the curve of $\chi^{\prime \prime}$ (see Equation $(3.19 b))$ versus $H_{0}$ (internal magnetic field) where $\chi^{\prime \prime}$ has decreased to half of its peak value. The damping factor, $\alpha$, is related to the linewidth, $\Delta H$, as explained by Equation (3.20).

\subsubsection{Effective linewidth $\Delta H_{e f f}$}

The insertion loss of the ferrite device is related to the magnetic loss in ferrites. The imaginary part of the permeability of the positive polarization $\chi^{\prime \prime}$ (or $\mu^{\prime \prime}$ ) indicates this loss. Generally, effective linewidth $\Delta H_{\text {eff }}$ increases when the gyromagnetic line 


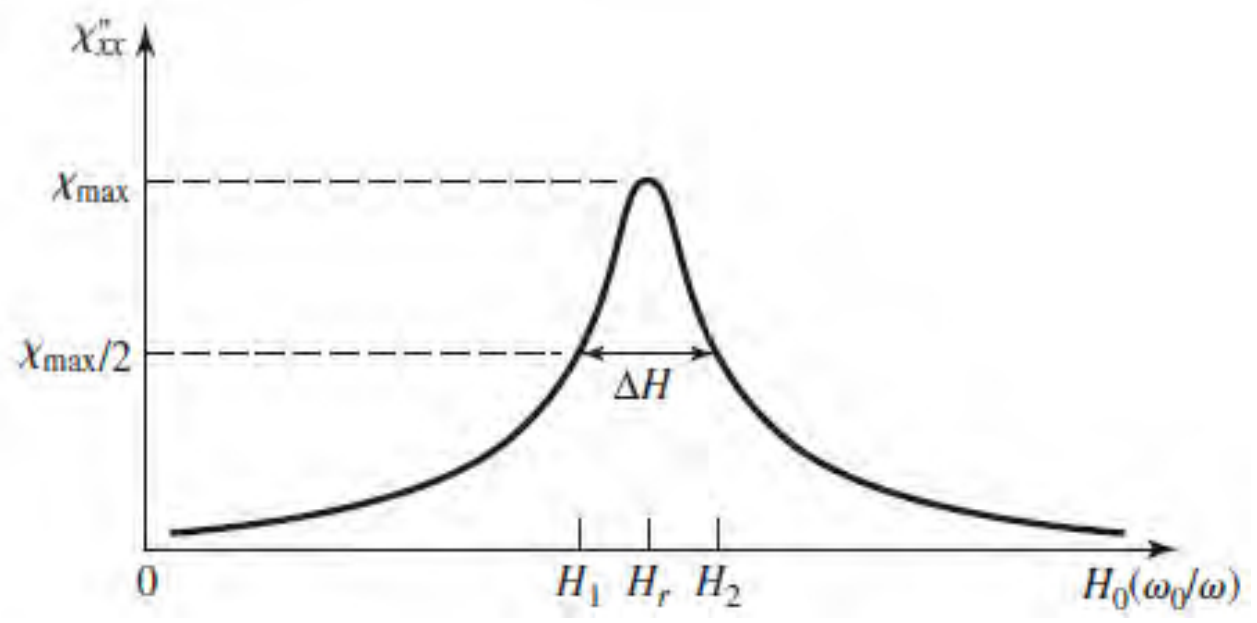

Figure 3.10: Definition of the linewidth $\Delta H[9]$

width $\Delta H$ increases.

We commonly use a Lorentz curve to model $\mu^{\prime \prime}$ far away from the resonance as shown in Figure 3.11 [14]. Accordingly, the effective line width $\Delta H_{e f f}$ is smaller than $\Delta H$. The reason why the line width is broadened when near the resonance is that the porosity and magneto crystalline anisotropy in ferrites increase the losses. Since the $\Delta H_{\text {eff }}$ is more accurate when the ferrite is far from resonance, there is much practical interest [14].

\subsubsection{Spin wave linewidth $\Delta H_{k}$}

Nonlinear phenomena could be observed when the microwave power exceeds a threshold. Non-linearity brings additional magnetic loss and generates other frequencies which are not desired in our devices. The effect of non-linearity is related to the magnetic microwave field $h_{c}$ which depends on the external dc magnetic field. The presence of the spin waves is usually considered as the reason of nonlinear effects, which is described by $\Delta H_{k}$ as shown in Figure 3.12. For a certain static magnetic field $\mathrm{H}$ denoted $H_{\text {sub }}$, there is a minimum of the microwave magnetic field $h_{c}$ related 


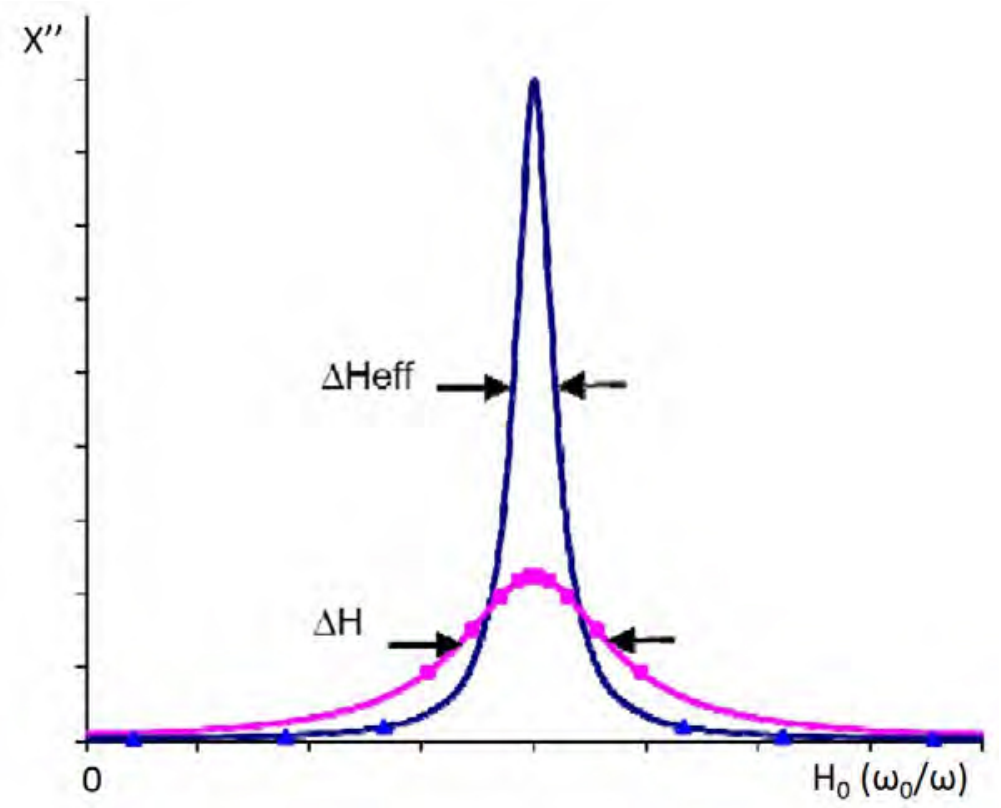

Figure 3.11: Definition of the effective linewidth $\Delta H_{\text {eff }}[14]$

to $\Delta H_{k}$ as explained by Equation (3.21) [14]. The high power capability could be

achieved by increasing the value of $\Delta H_{k}$. The typical value of $\Delta H_{k}$ is from 1 to more than 20. The relation between the line widths mentioned till now is $\Delta H_{k}<\Delta H_{\text {eff }}$ $<\Delta H$.

$$
h c_{\text {min }}=\frac{2 f \Delta H_{k}}{\gamma M_{s}}
$$

\subsubsection{Permittivity and loss tangent}

Besides the magnetic properties, the ferrite's dielectric properties are also important for microwave applications. The relative real permittivity $\epsilon^{\prime}$ determines the real wave-length and wave impedance in ferrites. The typical value is about 12 to 16 . Meanwhile, the relative imaginary part of the permittivity $\epsilon^{\prime \prime}$ or the dielectric loss tangent determines the dielectric loss. We usually measure the dielectric loss tangent 


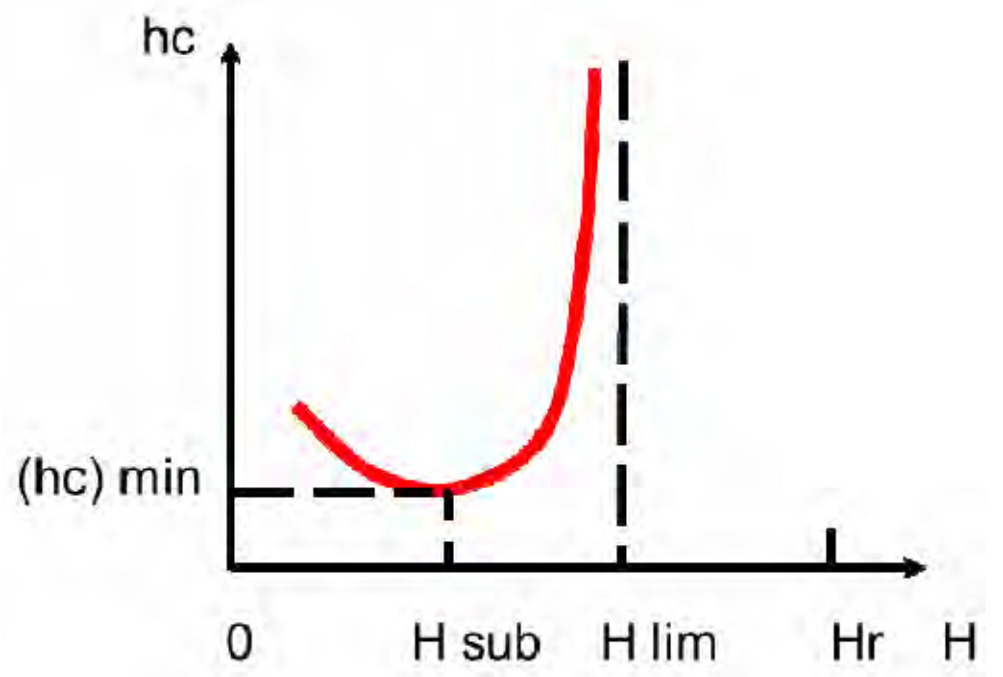

Figure 3.12: Definition of the spin wave linewidth $\Delta H_{k}$ [14]

$\tan \delta$ at $10 \mathrm{GHz}$. Generally, tan $\delta$ is small and ranges between $10^{-4}$ and $10^{-3}$ for most ferrimagnetic materials at $10 \mathrm{GHz}$. Therefore, the insertion loss of the device is more affected by the magnetic losses in this range [14].

\subsection{Ferrite LTCC}

\subsubsection{Introduction}

There are currently few commercially available ferrite LTCC materials, none special for microwave. The tape used in this thesis is ESL 40012 from ESL ElectroScience (USA) which could be a magnetic tape for high frequency applications with multilayers. These applications usually require a permeability, higher than 400 . The tape is a flexible cast film as shown in Figure 3.13 where magnetic powder is dispersed in an organic matrix. In order to get a dense body, we need a fired-temperature of $885^{\circ} \mathrm{C}$. We can print metallized circuits on each sheet of the tape, then a pressure/temperature combination of $14 \mathrm{MPa}$ and $70^{\circ} \mathrm{C}$ is used to laminate several sheets. 


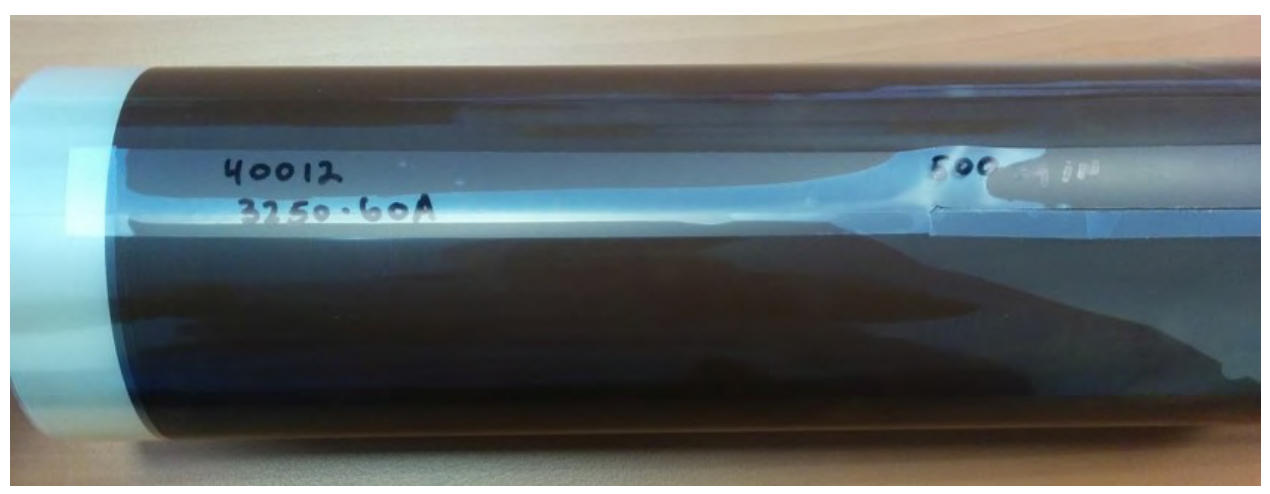

Figure 3.13: Photo of ESL 40012 ferrite tape ('green' tape)

A silicone-coated polyester film is also employed to protect the tape from being contaminated by the environment. The polyester film also improves the mechanical strength, and convenience of handling [93].

\subsubsection{Characterization of ESL 40012}

\section{Hysteresis curve}

The ferrite LTCC material ESL 40012 ferrite has been already characterized by previous works [15]. Magnetostatic characterization is performed by the measurements of hysteresis. Important ferrite characteristics such as the saturation magnetization, relative permeability, squareness and coercivity could be obtained. Two different types of transformers have been designed: 1. a straight solenoid transformer completely embedded within the ferrite LTCC material (Figure 3.14(a) [15]); 2. a toroid transformer designed in which unit cells are closely spaced and the fields are more tightly contained within the windings (Figure 3.14(b) [15]) .

When we inject a peak current of $650 \mathrm{~mA}$ into the winding, the measured hysteresis curves from the embedded transformers could be shown in Figure 3.15 [15]. The magnetic saturation $B_{s}$ is measured to be $0.33 \mathrm{~T}$ when a magnetic field of $2.1 \mathrm{KA} / \mathrm{m}$ 
is applied in the solenoid winding. The remanent flux density $B_{r}$ is $0.18 \mathrm{~T}$, the squareness (saturation remanence divided by saturation magnetization) is 0.55 , and the coercive magnetic field $H_{c}$ is $520 \mathrm{~A} / \mathrm{m}$. Similarly, we measured these parameters when toroid winding is used. However the turn density of the toroid is lower than that of the solenoid because of the different curvature of the toroid. The B-H curve for the toroid is shown in Figure 3.15 by a solid line.

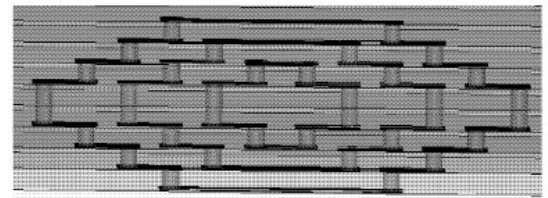

(a)

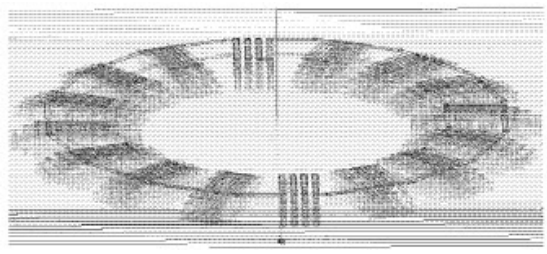

(b)

Figure 3.14: LTCC (a) solenoid transformer unit cell cross section and (b) toroid transformer tilted front view [15]

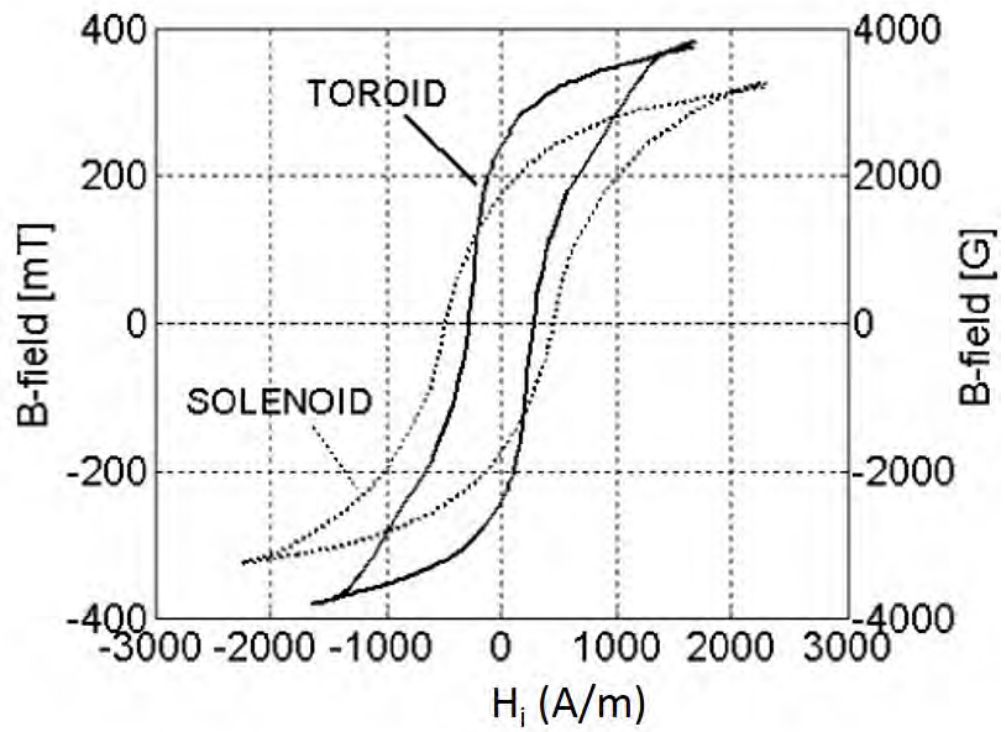

Figure 3.15: Measured hysteresis curves from the LTCC transformers [15] 


\section{Magnetic Saturation $M_{s}$}

Figure 3.16 [15] shows the curve B-H which could be used to model the non-linearity in Maxwell 3D. Although saturation could not quite be achieved only with the toroid, the trend clearly indicates that the magnetization saturation $\mu_{0} M_{s}$ approaches 400 $\mathrm{mT}$ instead of the $330 \mathrm{mT}$ obtained using the solenoid. A small internal H-field higher than $2500 \mathrm{~A} / \mathrm{m}$ is needed to saturate it. For the toroid, $B_{r}=250 \mathrm{mT}$, the squareness is approximately 0.63 , and $H_{c}=330 \mathrm{~A} / \mathrm{m}$ [15].

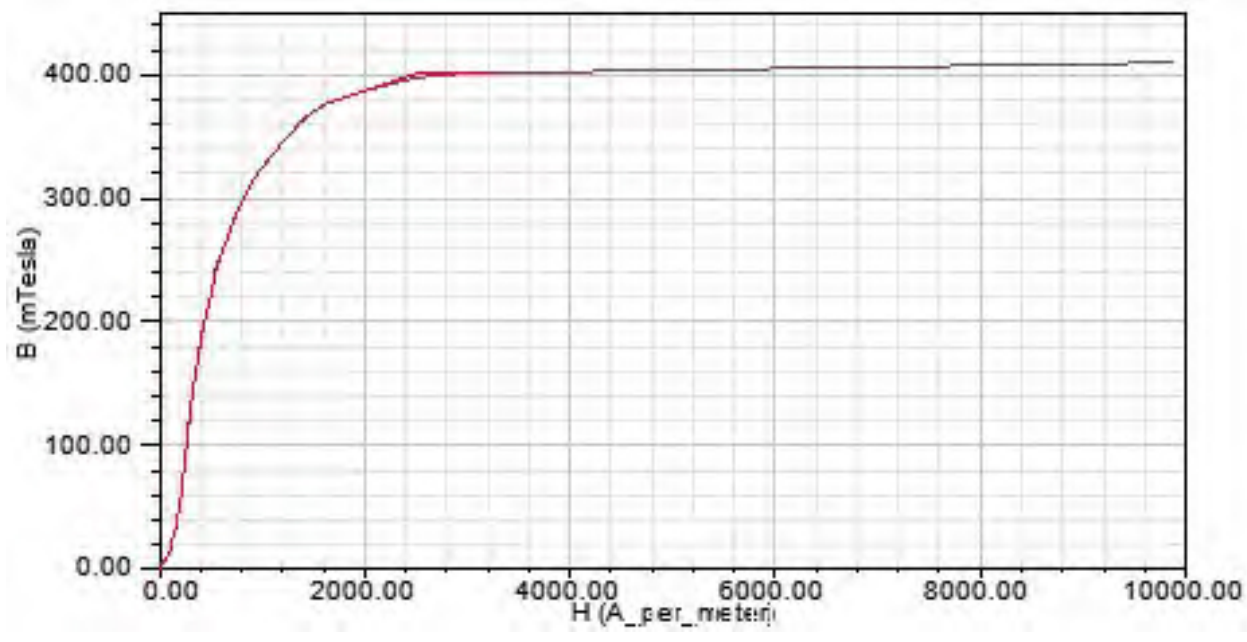

Figure 3.16: B-H curve (first magnetization) used in EM simulator

\section{Losses}

This material is very lossy below $10 \mathrm{GHz}$ due to the low-field losses, especially when it is completely demagnetized as shown in Figure 3.17 [15]. The magnetization frequency is defined as $f_{m}=\gamma \mu_{0} M s(11.2 \mathrm{GHz})$. So, according to the previous remarks, an operating frequency higher than $14 \mathrm{GHz}$ for our circulators is chosen far from the lossy frequency area to avoid low-field losses. Moreover, this material has a relative permittivity $\varepsilon_{r}=14.6$ and a dielectric loss tangent $4.24 \times 10^{-3}$. 
The damping factor $\alpha$ is found to be around 0.35 by measuring a CPW transmission line on ESL 40012 [94]. Hence the line width $\Delta H$ equals 250 Oe according to Equation (3.20) [9].

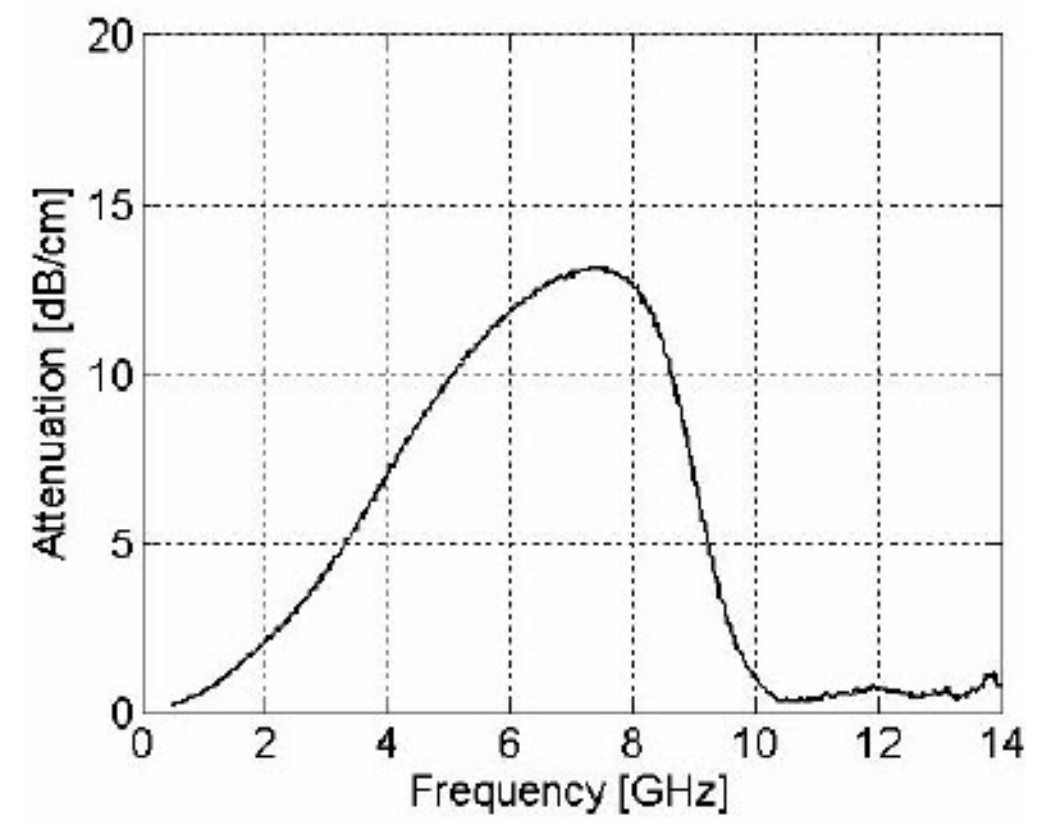

Figure 3.17: Attenuation constant in terms of frequency

\section{Curie Temperature $T_{c}$}

Ring samples using ESL 40012 LTCC tape with 64 layers were realized [95]. Then the $\mathrm{B}-\mathrm{H}$ curve measurements were performed in dependence on environment temperature as shown in Figure 3.18.

From the dependence of the maximum polarization with temperature, we can estimate the value of the Curie temperature to be higher than $100^{\circ} \mathrm{C}$. Saturation polarization, coercivity, as well as remanence vanish at temperatures above $T_{c}$. Due to the finite resolution of the measurement system the observation of the maximum polarization with temperature seems to give the most reliable estimate for $T_{c}$. The 


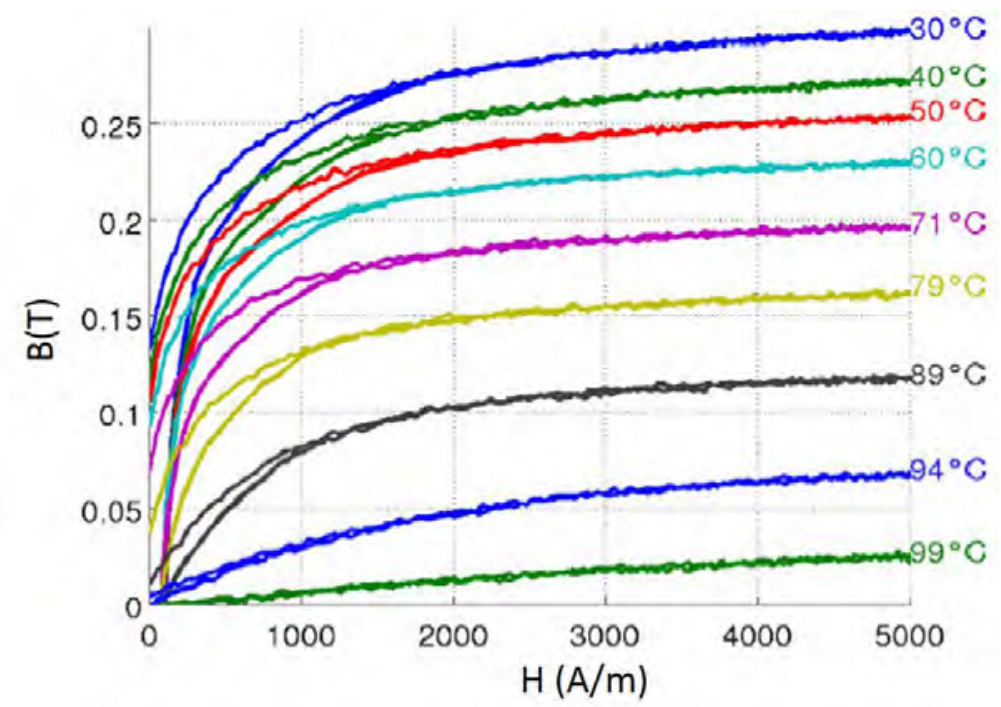

Figure 3.18: Major magnetization curves of the ESL 40012 sample at different sample temperatures, magnetic polarization $\mathrm{B}(\mathrm{T})$ versus magnetic field $\mathrm{H}(\mathrm{A} / \mathrm{m})$. The maximum magnetic field was $5 \mathrm{kA} / \mathrm{m}$ for all curves.

maximum magnetic magnetization $\mu_{0} M_{s}$ is $0.3 \mathrm{~T}$ which is in agreement with the measured $\mu_{0} M_{s}$ in Figure 3.15 when solenoid transformer was used. However, a measurement of VSM (Vibrating Sample Magnetometer) was also performed in our laboratory LT2C showing that the magnetization saturate $\mu_{0} M_{s}$ loses $50 \%$ at $100^{\circ} \mathrm{C}$.

\section{Other properties and summary}

The principle parameters are presented before such as the hysteresis curve, magnetization saturation $M_{s}$, losses and Curie temperature $T_{c}$. The list below concludes the characterization of ferrite LTCC ESL 40012 including some other physic fired tape properties [93].

- Relative permittivity: $\varepsilon_{r}=14.6$

- Loss tangent: $\tan \sigma=4.24 \times 10^{-3}$

- Magnetization saturation: $\mu_{0} M_{s}=0.4 \mathrm{~T}$ 


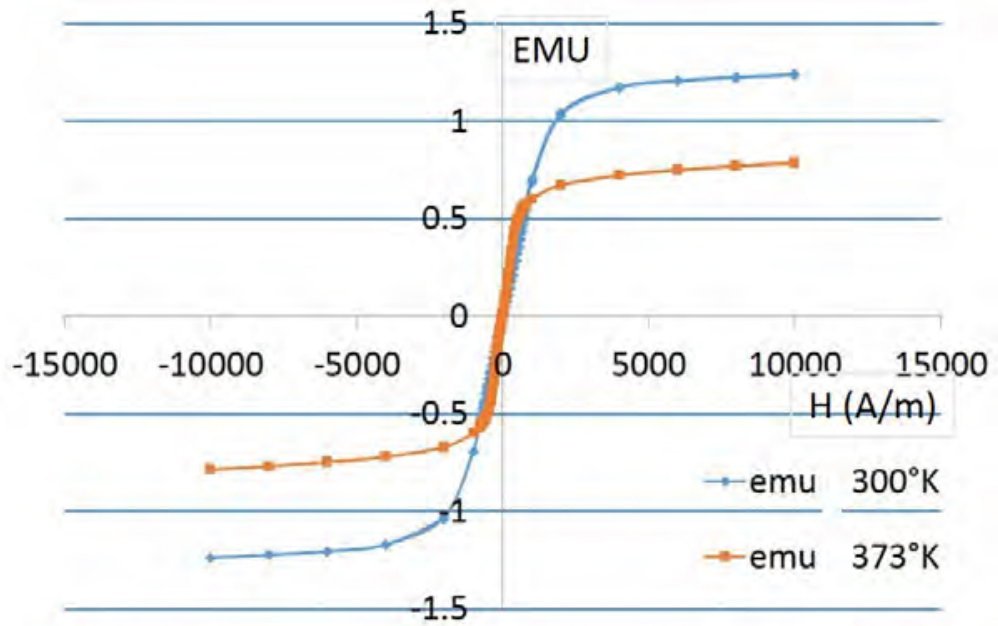

Figure 3.19: The VSM (Vibrating Sample Magnetometer) measurement performed by laboratory LT2C shows that the magnetization saturation $\mu_{0} M_{s}$ loses $50 \%$ at $100^{\circ} \mathrm{C}$

- Internal magnetic field to saturate the ferrite: $H_{i}>2500 \mathrm{~A} / \mathrm{m}$

- Linewidth: $\Delta H=250$ Oe

- B-H curve: Figure 3.16

- Remanence: $B_{r}=250 \mathrm{mT}$

- Coercivity: $H_{c}=330 \mathrm{~A} / \mathrm{m}$

- Curie Temperature: $T_{c} \geq 100^{\circ} C$

- Layer thickness: $\mathrm{t}=110 \mu \mathrm{m}$

- Insulation resistance: $\geq 10^{8} \Omega$

- Thermal Coefficient of Expansion: $9.4 \mathrm{ppm} /{ }^{\circ} \mathrm{C}$

- Breakdown voltage: $1000 \mathrm{~V} / 25 \mu \mathrm{m}$

- Fired shrinkage (x and y): 17\% 
- Fired shrinkage (z): $16.5 \%$

- Fired density: $5.35 \mathrm{~g} / \mathrm{cm}^{3}$

\subsubsection{Rf behavior of saturated ferrite}

Circulator's functionality lies on the ferrite material, so it is necessary to study the properties of ferrites to find the operating point of our circulators. As said before, the working frequency of our circulators is assumed to be higher than $14 \mathrm{GHz}$. In order to explain the ferrite's rf behavior with different DC magnetic fields and magnetization saturations, the elements $\kappa$ and $\mu$ in Polder tensor are normalized by the operating frequency leading to the property of the ferrite itself (independent of frequency). Two variables are then defined:

- $\sigma=\gamma \mu_{0} H_{i} / \omega$ which shows the normalized strength of the internal magnetic field $H_{i}$

- $\mathrm{p}=\gamma \mu_{0} M_{s} / \omega$ which shows the normalized magnetization saturation of the ferrite $M_{s}$

Then the elements $\kappa$ and $\mu$ of the permeability tensor can be reformed by Equations (3.22a) and (3.22b) where $\alpha$ is the damping factor. The $\mu_{\text {eff }}$ and $\frac{\kappa}{\mu}$ can also be expressed in terms of $\mathrm{p}$ and $\sigma$ as shown by Equations (3.22c) and (3.22d).

$$
\begin{gathered}
\mu=1+\frac{p(\sigma+j \alpha)}{(\sigma+j \alpha)^{2}-1} \\
\sigma=\frac{p}{(\sigma+j \alpha)^{2}-1} \\
\mu_{e f f}=\frac{\mu^{2}-\kappa^{2}}{\mu}=1+\frac{p \sigma}{\sigma^{2}-1}-\frac{p^{2}}{\left(\sigma^{2}+p \sigma-1\right)\left(\sigma^{2}\right)-1}
\end{gathered}
$$




$$
\frac{\kappa}{\mu}=\frac{p}{\sigma^{2}+p \sigma-1}
$$

Figure 3.20 shows that when $\sigma=1$, the material is very lossy due to the resonance $\left(\omega=\omega_{0}\right)[9]$. Isolators can be designed based on this resonance. The zone beyond $\sigma$ $=1$ is called the high $H_{i}$ zone and the zone below $\sigma=1$ is called the low $H_{i}$ zone. Either high or low $H_{i}$ zone could be used to operate a circulator [82]. High $H_{i}$ means more powerful external magnet should be applied leading to a bigger size and more weight of the device.
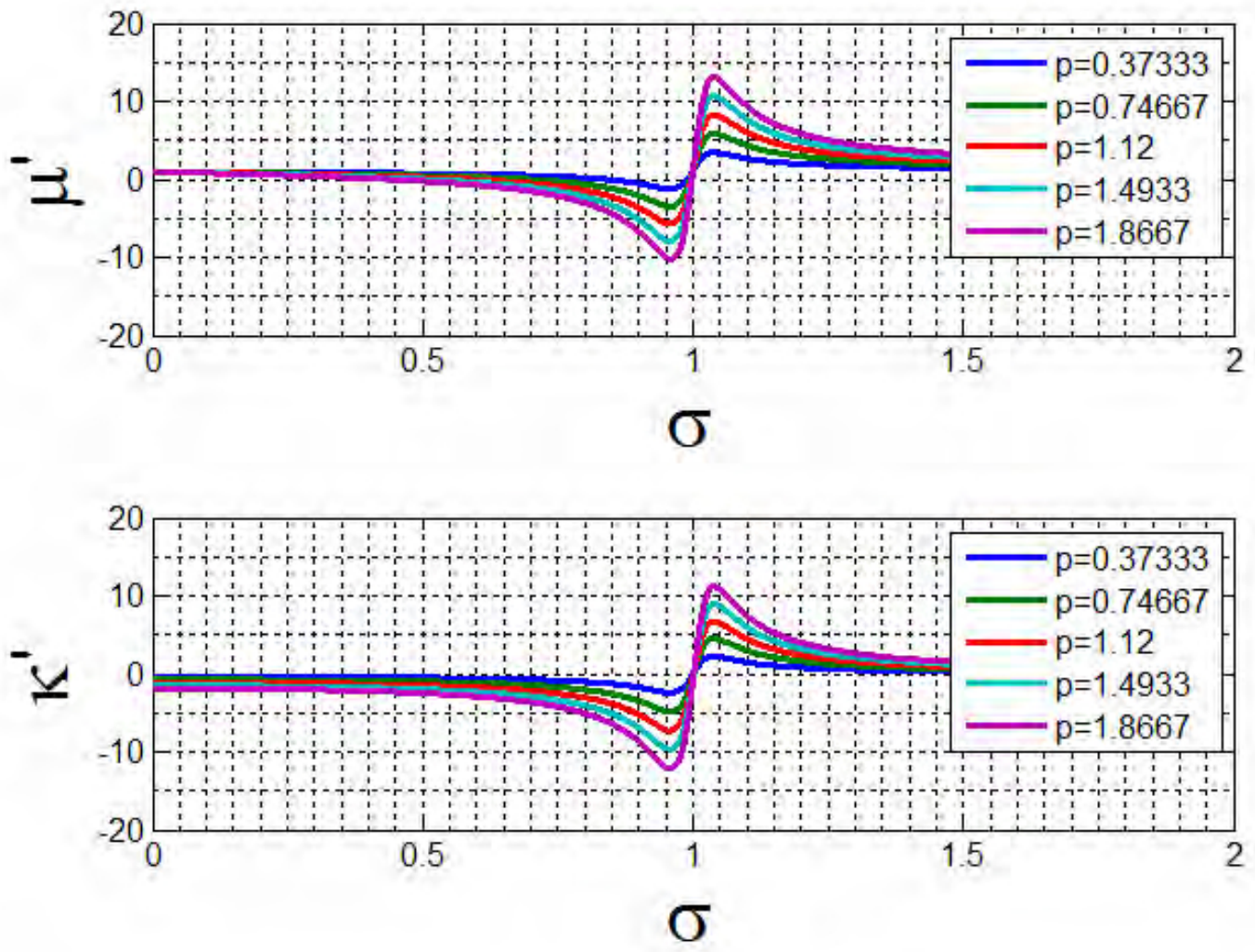

Figure 3.20: $\mu^{\prime}$ and $\kappa^{\prime}$ in terms of $\sigma$

The second part (losses) of $\mu$ and $\kappa$ along with the $\sigma$ is shown in Figure 3.21. It demonstrates that the loss increases with the increase of the p. This is the reason 
why a low $M_{s}$ ferrite should be used to design a low frequency circulator in order to avoid unnecessary losses. In the vicinity of the resonance, the attenuation is indeed strong. Moreover, in high $H_{i}$ zone, the $\mu^{\prime \prime}$ and $\kappa^{\prime \prime}$ could be smaller than those in low $H_{i}$ zone, which could be used to design low-loss devices.
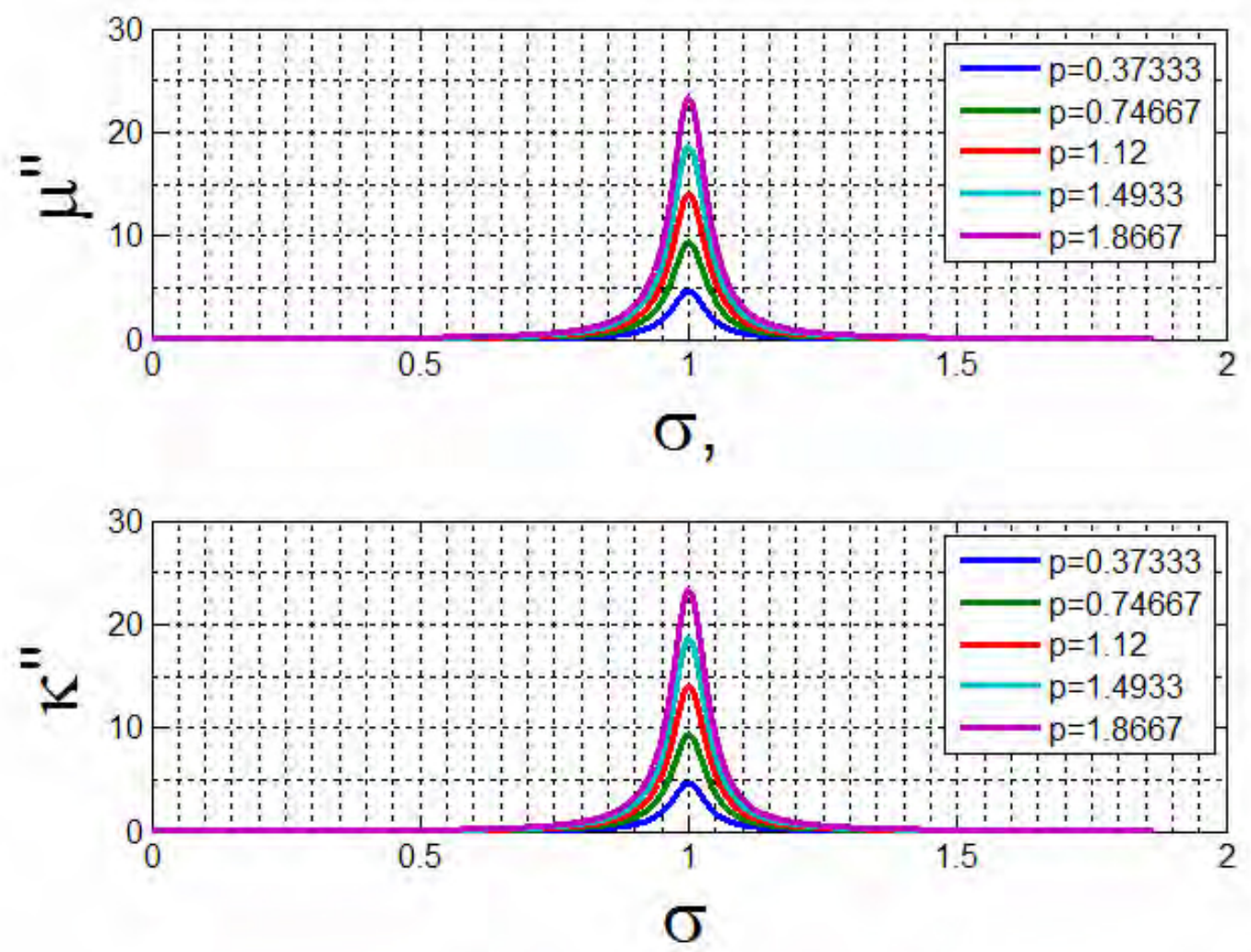

Figure 3.21: $\mu^{\prime \prime}$ and $\kappa^{\prime \prime}$ in terms of $\sigma$

In practice, the $\mu_{\text {eff }}$ and $\kappa_{\text {eff }}$ are more important, because they take into account the interaction between the ferrite and the rf signals. Figure 3.22 shows that the real resonance frequency shifts towards the left, which makes the low $H_{i}$ zone smaller compared with that in Figure 3.20. In addition, the low $H_{i}$ zone further shrinks with the increase of the $\mathrm{p}$, which explains why high $M_{s}$ ferrite is not suitable for low frequency circulator when using a low $H_{i}$ injection. Besides, the $\mu_{\text {eff }}^{\prime}$ becomes 
negative when $\mathrm{p}>1$ in low $H_{i}$ zone, which means the cut-off mode. So a ferrite with too high $M_{s}$ doesn't work in low $H_{i}$ zone. However, in high $H_{i}$ zone, $\mu_{e f f}$ is always positive which allows to design a circulator even a high $M_{s}$ ferrite is used.
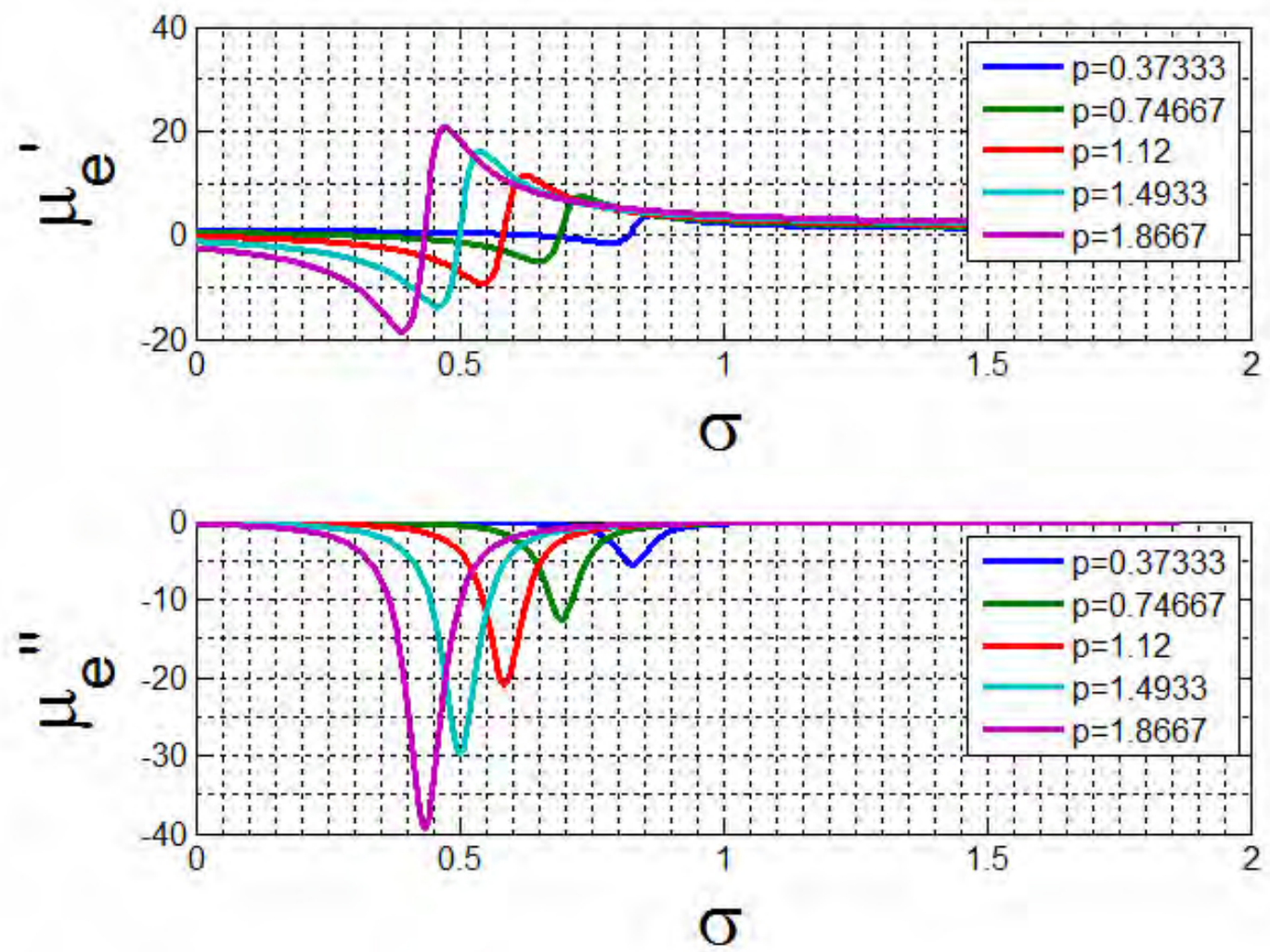

Figure 3.22: $\mu_{e f f}$ in terms of $\sigma$

Figure 3.23 shows the $\frac{\kappa}{\mu}$ which indicates the capability of the non-reciprocity. As expected, higher p introduces more non-reciprocity which could be used to design high frequency circulators, but it introduces more losses. That is why it is a challenge to design high frequency circulators with low losses.

In conclusion, ESL 40012 ferrite tape has a $\mu_{0} M_{s}=0.4 \mathrm{~T}$, therefore, $\mathrm{p}=0.74667$ which is the green line when frequency $\mathrm{f}=15 \mathrm{GHz}$ ( or $\mathrm{p}=0.8$ when $\mathrm{f}=14 \mathrm{GHz}$ ). The 

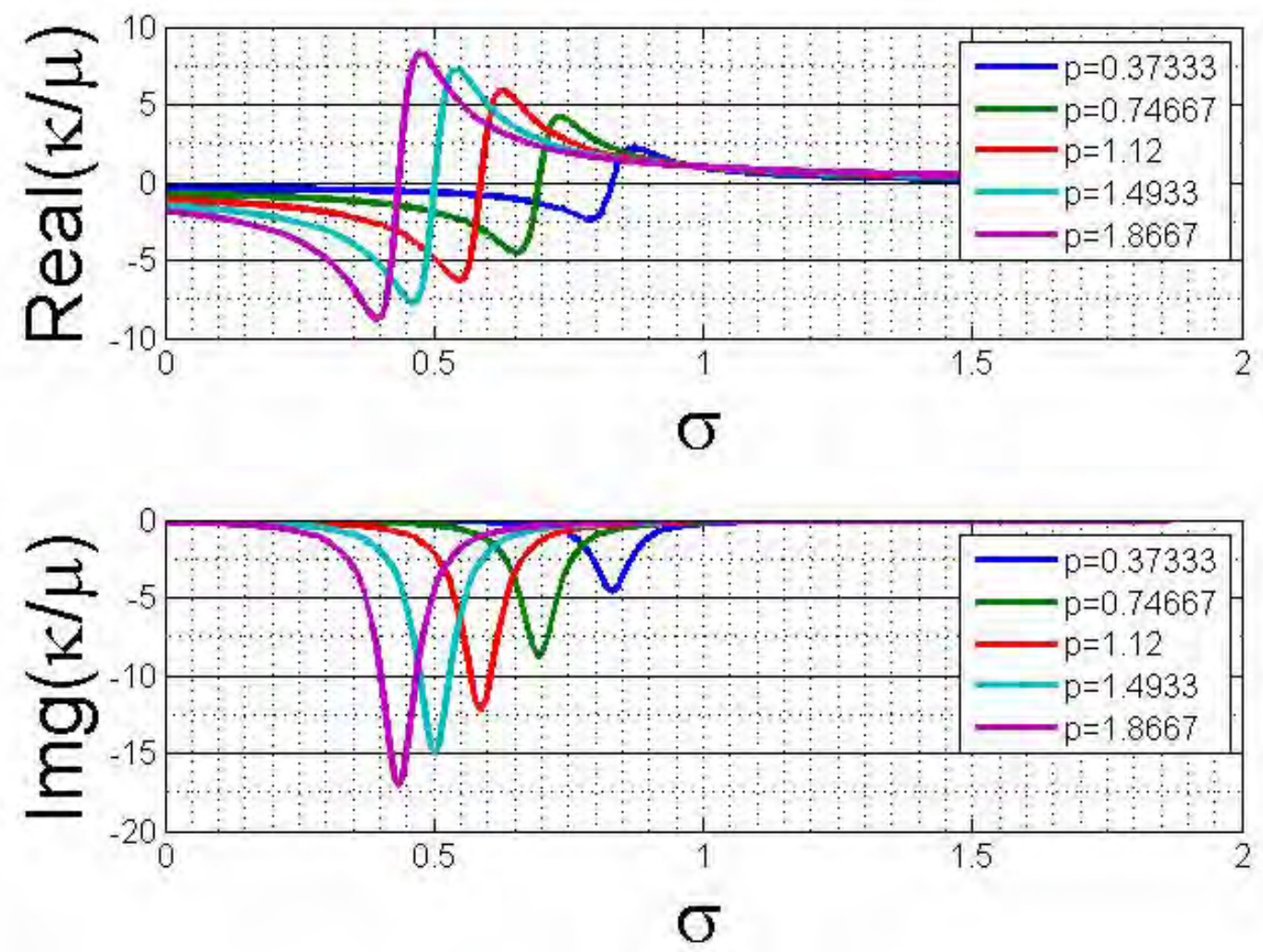

Figure 3.23: $\kappa$ on $\mu$ in terms of $\sigma$

low $H_{i}$ zone is then chosen to use a small internal magnetic field and have a relatively low loss.

\subsubsection{Rf behavior of partially magnetized ferrite}

Typically, external magnetic field intensity $\mathrm{H}$ is in order of $80 \mathrm{kA} / \mathrm{m}$ to saturate the ferrites and required magnets having large sizes make the system extremely bulky and unsuitable for compact $\mathrm{SiP}$ applications. An elegant solution to this problem of bulky electromagnets is to embed the bias windings into the ferrite substrate itself leading to the ferrite partially magnetized. Therefore, in this section, we will study the rf behavior of partially magnetized ferrites. The ferrite ESL 40012 has a $\mu_{0} M_{s}=400$ 
$\mathrm{mT}$ and the partially magnetized ferrite model 'Green and Sandy' (Equations (3.14a) to (3.14f)) is used to estimate the elements of the permeability tensor.

Figure 3.24 shows that the $\kappa$ and $\mu$ increase with the increase of the internal magnetization $M$ when $\mu_{0} M_{s}=400 \mathrm{mT}$. As discussed before, $\frac{\kappa}{\mu}$ signifies the nonreciprocity, so higher magnetization introduces more non-reciprocity as demonstrated in Figure 3.25. The fact that $\mu_{e f f}$ is a function of magnetization could be used to analyze the frequency variation of our ferrite LTCC circulator with integrated winding later.
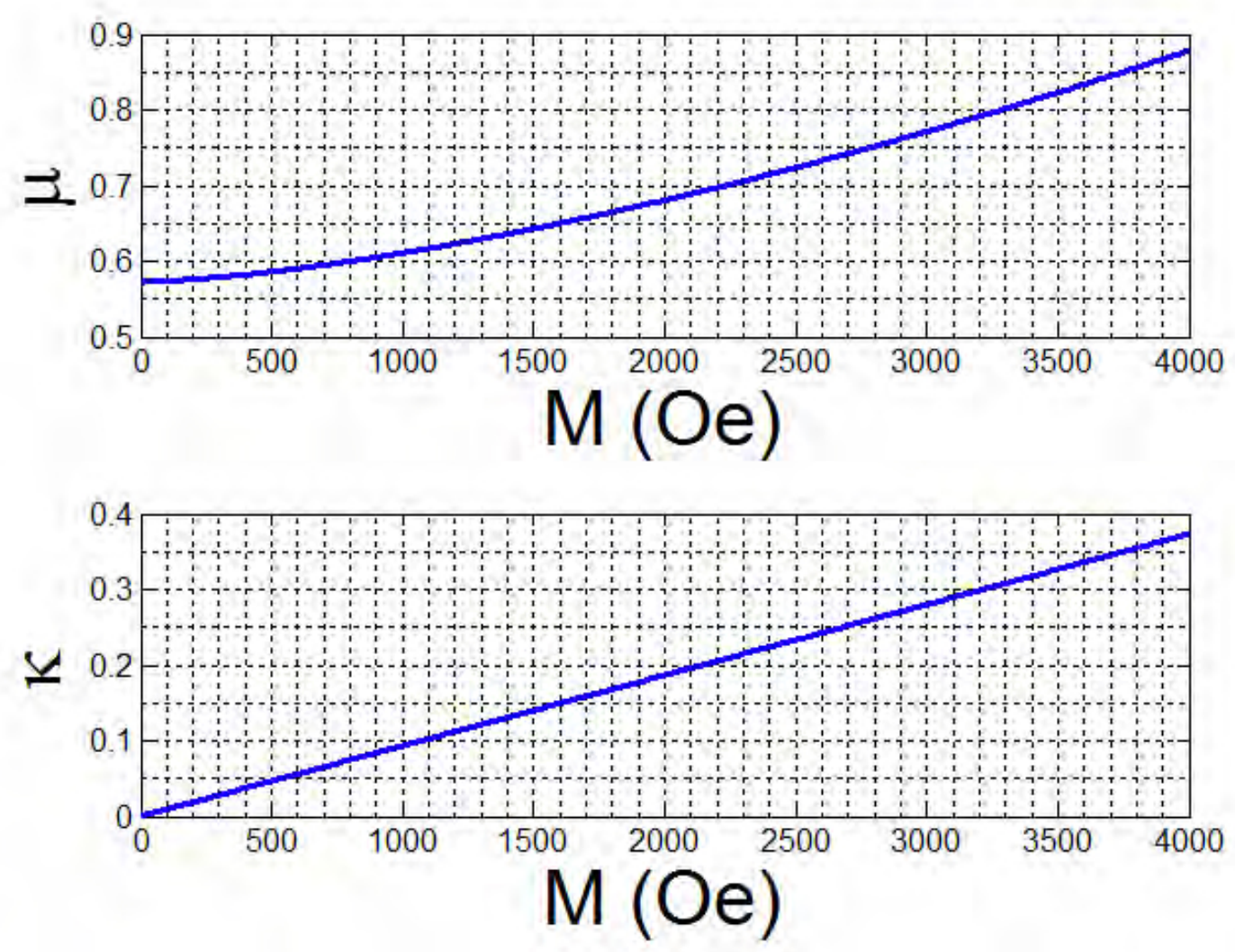

Figure 3.24: $\kappa$ and $\mu$ in terms of internal magnetization $M$ 

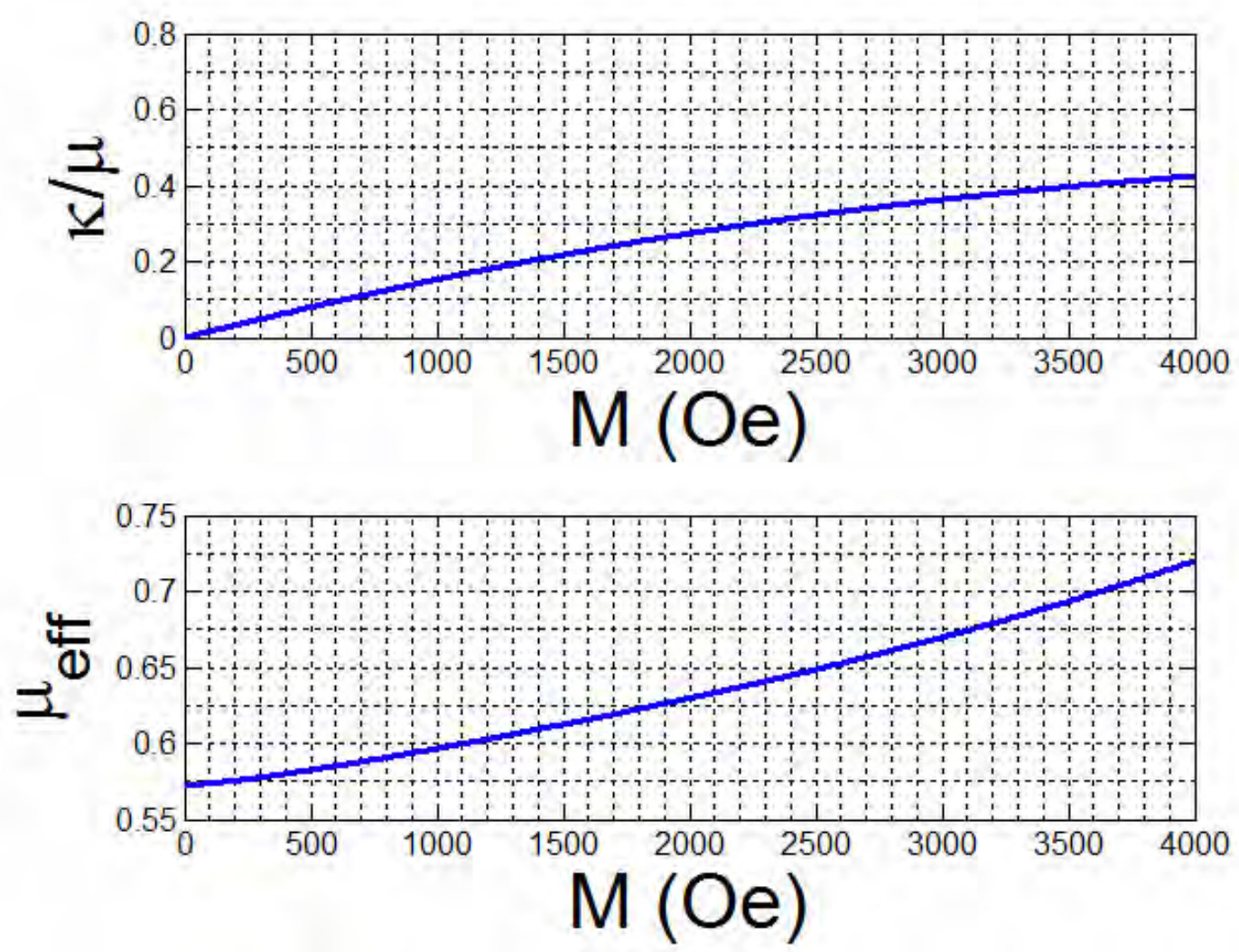

Figure 3.25: $\frac{\kappa}{\mu}$ and $\mu_{e f f}$ in terms of internal magnetization $M$

\subsubsection{Conventional LTCC fabrication process}

Although the fabrication of ferrite LTCC devices may be different from that of the conventional LTCC devices, the principle procedures of fabrication are almost the same [96]. The basic manufacturing process for multilayer LTCC substrates is shown in Figure 3.26 [16]. A milky slurry is made by mixing the ceramic/magnetic powder and organic binder. The green sheet of the LTCC is then obtained by casting the slurry into the tape and cut by a doctor blade. This raw sheet is a paper-like flexible film. We can now print the desired circuits on the sheet and realize the vias. After aligning all the sheets of LTCC, a combination of temperature and pressure is used to laminate them. Finally the device is fired, according to the material's profile and 


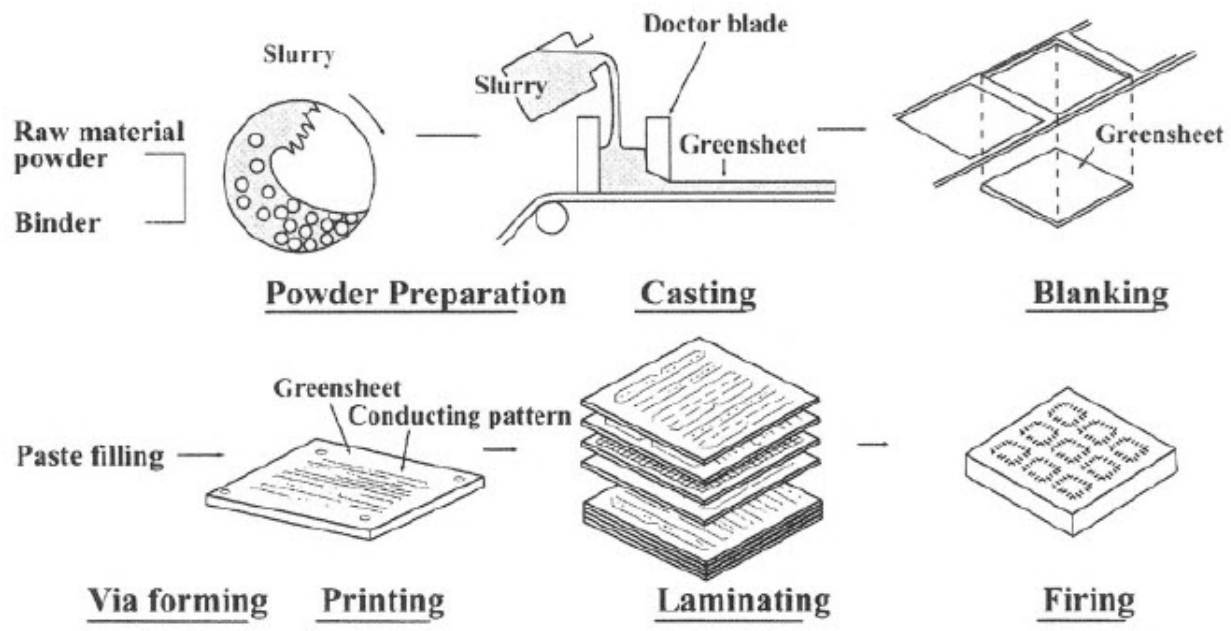

Figure 3.26: Typical multilayer ceramic substrate manufacturing process [16]

get the final structure. The challenge is the different shrinkage rates of materials such as metal and ceramic. The variation of temperature, pressure, lamination quality etc will directly affect the final device [16].

\subsubsection{Recent ferrite LTCC research works}

Ferrite Low Temperature Co-fired Ceramic (LTCC), which is originally used to design inductors, now could be used in high frequency applications. This section will present some recent ferrite LTCC work.

The ferrite LTCC tape from Ferro Corporation was firstly characterized using circuits that are fabricated by Dr Joey R. Bray, Dr. Kari T. Kautio and Dr. Langis Roy [96] in 2004. This work has demonstrated that high quality ferrite LTCC circuitry can be fabricated using standard processing techniques, which is an essential requirement for low cost high volume production.

In 2007, another commercial ferrite LTCC tape ESL 40012 (ESL ElectroScience) was characterized in [15]. The measured relative permittivity $\epsilon_{r}$ is 13.6 at $9.86 \mathrm{GHz}$. The $\tan \delta$ values is 0.004 and 0.001 at $9.86 \mathrm{GHz}$ and $27.2 \mathrm{GHz}$ respectively. When 
toroid winding is integrated in the ferrite LTCC, the measurement extracts a magnetic saturation $\mu_{0} M_{s}$ of $400 \mathrm{mT}$, with a remanence of $250 \mathrm{mT}$, and a coercivity of 330 $\mathrm{A} / \mathrm{m}$. The approximate linear relative permeability is 370 .

Tunable antennas for SoP (system on package) application were then developed [27]. The winding embedded in the ferrite could produce a controllable magnetostatic field which could make the antenna's frequency tunable. Due to the lack of the demagnetization factor, the embedded winding greatly reduces the required bias field for a frequency tuning by over 95\%. This property makes ferrite LTCC an ideal substrate for tunable system-on-package applications because we don't need bulky electromagnets.

Then a theoretical model was described to explain the partially magnetized ferrite antenna's frequency tuning [28]. The authors use the theoretical model of Green and Sandy to calculate the terms of the permeability tensor when the ferrite is partially magnetized. Then these values are used to predict the antenna's frequency shifting. Measurement shows the agreement between the calculation and simulation.

Moreover, a helical antenna has been developed using ten layers of ferrite LTCC. The helical winding is designed to act as antenna and bias coil at the same time [97]. This idea firstly reduces the size of the antenna because external bias could be removed. Besides, the integrated winding could avoid demagnetization factor, hence reduce the required tuning dc magnetic field. The measurement shows a tuning range of $10 \%$ of the operating frequency $(13 \mathrm{GHz})$. The design is compact, light-weight, low cost and highly suitable for tunable microwave systems .

Besides, a half mode substrate-integrated waveguide (SIW) phase shifter based on ferrite LTCC was presented in [98]. Similar to the tunable antenna's design, the biasing is realized by integrating a winding in the ferrite LTCC substrate. This removes the external electromagnets and avoids wasting energy caused by demagnetization 
field. The ferrite is partially magnetized by the winding, hence the required bias fields have been greatly reduced. The measurement demonstrates that a phase shift of $83.2^{\circ}$ and a figure of merit of $83.2^{\circ} / \mathrm{dB}$ could be achieved at the operating frequency of $13.1 \mathrm{GHz}$.

Finally, the heat effects created by embedded windings were studied [99]. By a number of simulations and measurements, the authors get a model which has a good agreement with the measurement. The study shows that the center frequency of the filter could be shifted by about $1 \mathrm{GHz}$ when we heat the device externally from $0{ }^{\circ} \mathrm{C}$ to $190{ }^{\circ} \mathrm{C}$. Besides, when the integrated winding is energized by $260 \mathrm{~mA}$, the internal temperature is about $250{ }^{\circ} \mathrm{C}$ leading to a frequency shifting more than $2 \%$.

Circulators, as one of the important ferrite devices, have never been designed on the ferrite LTCC substrate. This is the principal purpose of this thesis. A winding could be integrated into the circulator's structure to achieve multi-functionality. Chapter 4 will present our work on the circulator design, winding design and the measurements.

\subsection{Conclusion}

This chapter has highlighted the different types of magnetic materials, including diamagnetic, paramagnetic, ferromagnetic, antiferromagnetic and ferrimagnetic materials. Diamagnetic materials have a negative magnetization against the applied magnetic field leading to a small and weak susceptibility. However, paramagnetic materials could be attracted by an externally applied magnetic field because of a small and positive susceptibility. Ferromagnetism is basically used to form permanent magnets because all moments of individual atoms could be aligned resulting in a large susceptibility. Antiferromagnetic materials have mixed (unpaired) parallel and antiparallel 
aligned magnetic moments with equal amplitude, while the total net magnetization is still small. Ferrimagnetic materials have unequal antiparallel aligned magnetic moments leading to a large susceptibility and a saturation magnetization. Ferrimagnetic materials are non-conducting, so they are used in microwave devices.

Since ferrimagnetic materials are widely used in microwave devices, we introduce more about the ferrite. Spinel ferrites are among the most important mineral oxides and a source of metals in the extractive industry. The lodestone or magnet, is the most abundant natural magnetic spinel. The garnet structure is cubic and the most common form is the iron garnet and yttrium $\mathrm{Y}_{3} \mathrm{Fe}_{5} \mathrm{O}_{12}$, known as YIG (Yttrium Iron Garnet). Permanent magnets and high density magnetic recording medias are two main applications based on the M-type hexagonal barium hexaferrite or BaM.

After talking about the different types of ferrite, we focus on the basic properties of ferrite materials. As a non-reciprocal magnetic material, a permeability tensor is used instead of a constant. Polder model is the most common known model which explains the behavior of ferrite under saturated condition. Schlömann developed a model for demagnetized ferrites. Then Rado developed a theory which provides a good approximation for the extra-diagonal term $\kappa$, but inaccurate values for the diagonal ones $\left(\mu=\mu_{z}=1\right)$. By using experimental characterization cells, Green and Sandy have measured all the tensor components as a function of the ferrite magnetization state. Igarashi and Naito presented a formula for the transverse diagonal term in a partially demagnetized state ferrite. This model is a further improvement of the tensor Schlömann. To describe the permeability tensor of the ferrites (saturated, partially magnetized or demagnetized) correctly, a generalized approach was developed by Gelin, Quéffélec and other researchers. We also present the demagnetization factor, because the internal bias field $H_{0}$ is generally different from the externally applied field $H_{a}$. When the damping factor is considered, the loss of ferrites could be calculated, 
leading to the definition of linewidth including the linewidth $\Delta H$, effective linewidth $\Delta H_{\text {eff }}$ and spin wave linewidth $\Delta H_{k}$. Hysteresis loop is then described resulting in the definition of magnetization $M_{s}$, retentivity, residual magnetism, coercive Force, maximum energy product (BHmax) and squareness.

Ferrite LTCC is next presented. In particular, the characterization of ESL 40012 ferrite tape is introduced. We show the principle parameters including the hysteresis curve, magnetization saturation $M_{s}$, losses and Curie temperature $T_{c}$ along with other physic fired tape properties. The rf behaviors of both saturated and partially magnetized ferrite are predicted by our Matlab program. Finally the recent ferrite LTCC research works are presented varying from the characterization of the substrate to tunable antennas and phase shifters designs.

In the next Chapter, we will demonstrate how to design ferrite LTCC circulators based on this ESL 40012 substrate. 


\section{Chapter 4}

\section{Modeling and realizations of circulators}

\subsection{Introduction}

The design of a stripline Y-junction circulator is generally based on the work of $\mathrm{H}$. Bosma [5]. Usually, a stripline Y-junction circulator is made of two metal plane grounds, a center conductor with three ports and a ferrite disk surrounded by dielectric materials. For this conception, ferrite materials at microwave frequencies are usually operated under the saturation state and Polder equations are employed to calculate the permeability tensor by taking into account the applied static magnetic filed $H_{0}$, the magnetization $M_{s}$, the operating frequency and the damping factor $\alpha$ (or $\Delta H$ ), etc [37]. However, partially magnetized ferrite has advantages in frequency tunability by varying internal magnetization $\mathrm{M}$ easily.

In this chapter, a CPW transmission line on ferrite LTCC is firstly realized to have an intimate knowledge of ferrite LTCC design, then different structures of circulators such as stripline, microstrip and coplanar structures are described, followed by the theory and mathematical calculations of a stripline junction circulator. Three ferrite LTCC circulators are finally presented: one is with external magnets and Polder equations are applied, one is with an integrated winding employing Green and Sandy's 
partially magnetized model and the last one is a wide-band circulator by stacking two circulators with different operating frequencies.

\subsection{CPW on ferrite LTCC}

\subsubsection{Introduction}

Since the ferrite LTCC technology is totally new to us, we start by design a simple CPW transmission line on it. The design of the CPW line is shown in Figure 4.1 and the fabricated one in Figure 4.2. Five layers are used and the total size $\left(\mathrm{L}^{*} \mathrm{~W}^{*} \mathrm{H}\right)$ is $15 \mathrm{~mm} * 4.79 \mathrm{~mm} * 0.55 \mathrm{~mm}$. Simulations under different dc bias fields are performed to demonstrate the resonance frequencies in terms of the applied field. The measurements are performed with an external electromagnet. The CPW transmission line follows the axis $\vec{x}$. The two ends of the CPW are connected to the probe station, while the electromagnet is placed in $\vec{y}$ direction, therefore the device is biased transversely as shown in Figure 4.3.

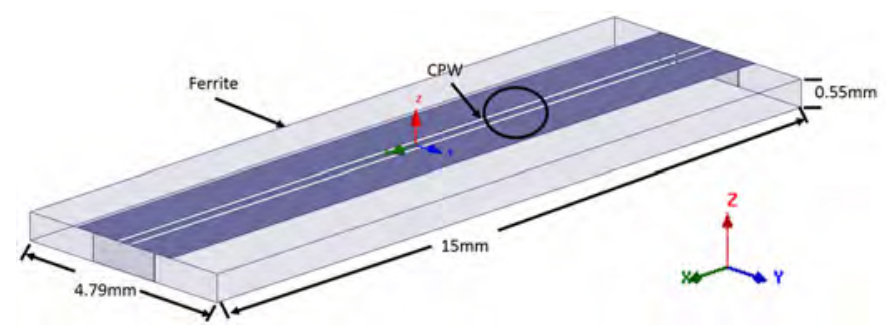

Figure 4.1: Design of a CPW on ferrite LTCC 


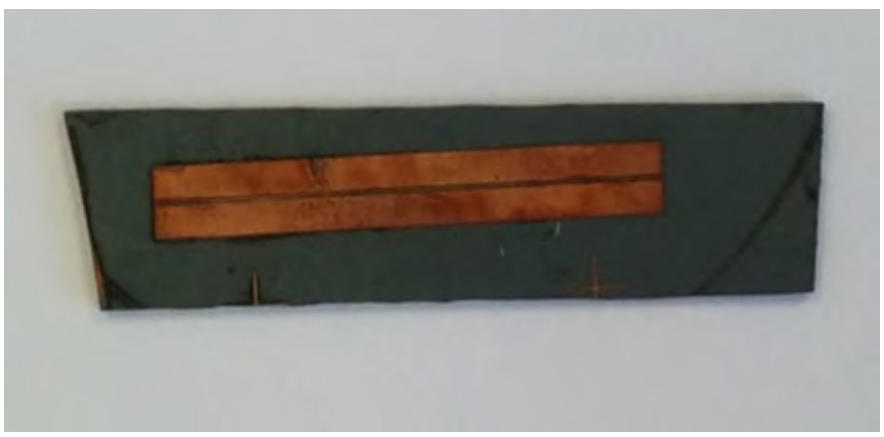

Figure 4.2: Photo of a fabricated CPW on ferrite LTCC

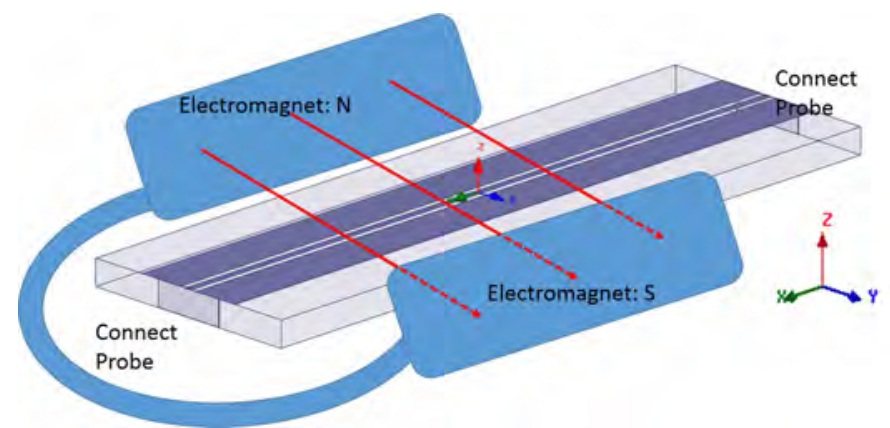

Figure 4.3: Measurement configuration of the CPW with an external electromagnet

\subsubsection{Demagnetized ferrite $H_{i}=0$}

When the ferrite LTCC is totally demagnetized, there is a low field loss below $10 \mathrm{GHz}$ according to the characterization in [15]. The measurement performed by Dr. Didier Vincent confirms this loss as shown in Figure 4.4.

However, the simulator HFSS cannot predict it. Figure 4.5 shows a perfect transmission in HFSS because it considers the demagnetized ferrite as a dielectric material $\left(M_{s}=0, \mathrm{M}=0\right.$ and $\left.H_{i}=0\right)$.

\subsubsection{Ferrite biased transversely}

When the ferrite is biased in $\vec{y}$ direction, the non-reciprocity could be observed.

Figures 4.6a and 4.6b show the comparison of simulated and measured S-parameters 


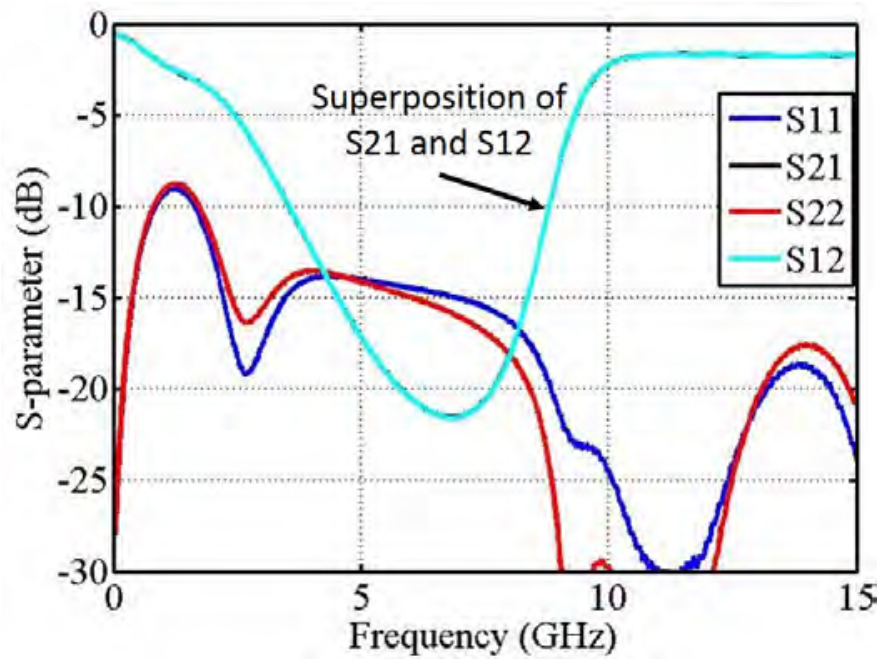

Figure 4.4: Measured S-parameters when ferrite is totally demagnetized

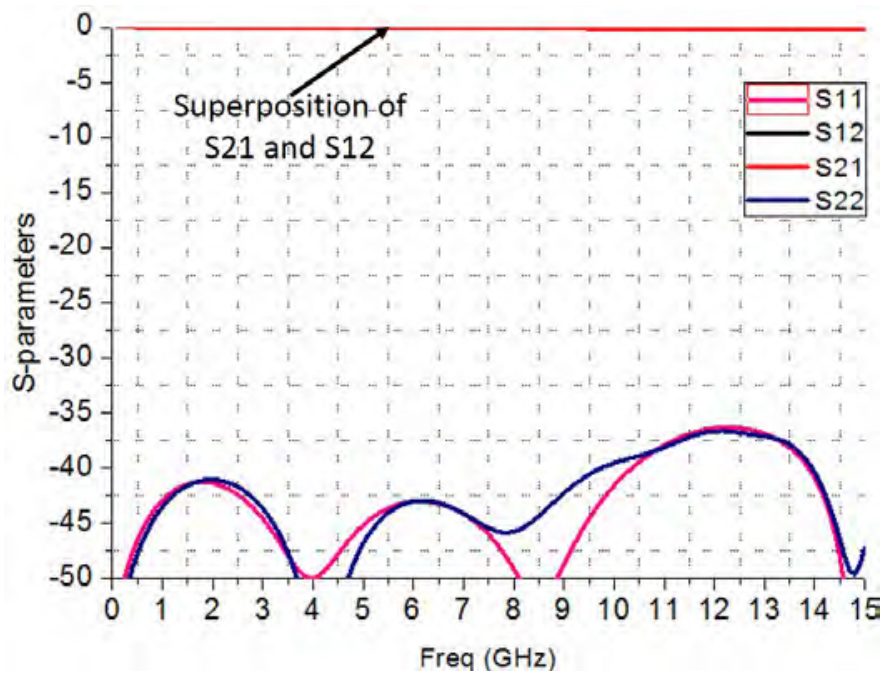

Figure 4.5: Simulated S-parameters when ferrite is totally demagnetized

when the ferrite is biased transversely with an internal magnetic field $H_{i}=52 \mathrm{KA} / \mathrm{m}$ (660 Oe). A magnetometer is used to measure the dc bias field near the CPW transmission line. Since a tangential bias is applied, the demagnetization factor is weak and negligible. In general, the simulated S-parameters meet the measured ones, except that the measured resonance frequency is $1.5 \mathrm{GHz}$ lower than the simulated one. This is probably due to the non-uniform magnetic bias fields hence the inaccurate 


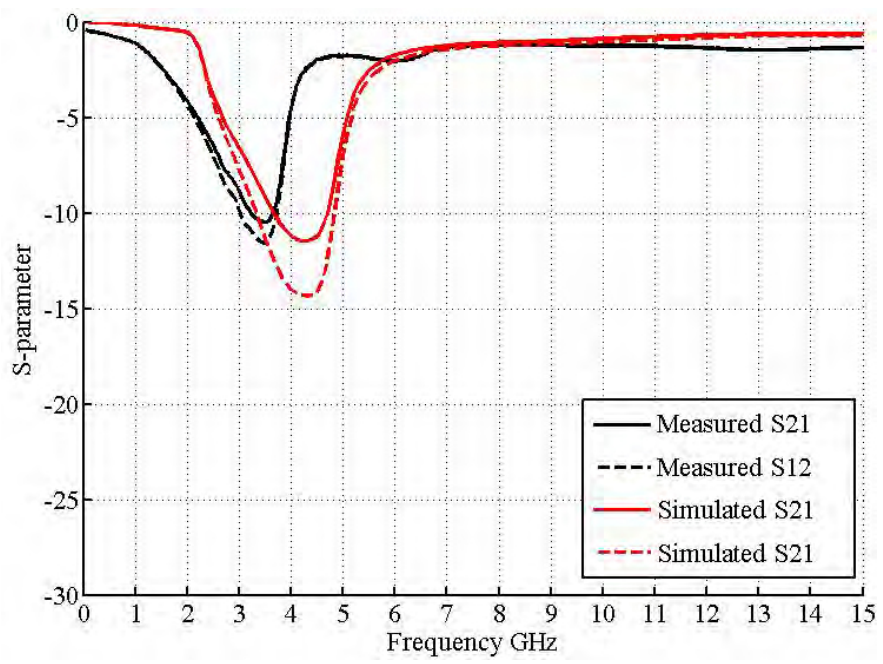

Figure 4.6a: Comparison of S21 and S12 between simulation and measurement when ferrite is biased transversely with internal magnetic field $H_{i}=660$ Oe

measurements by the magnetometer.

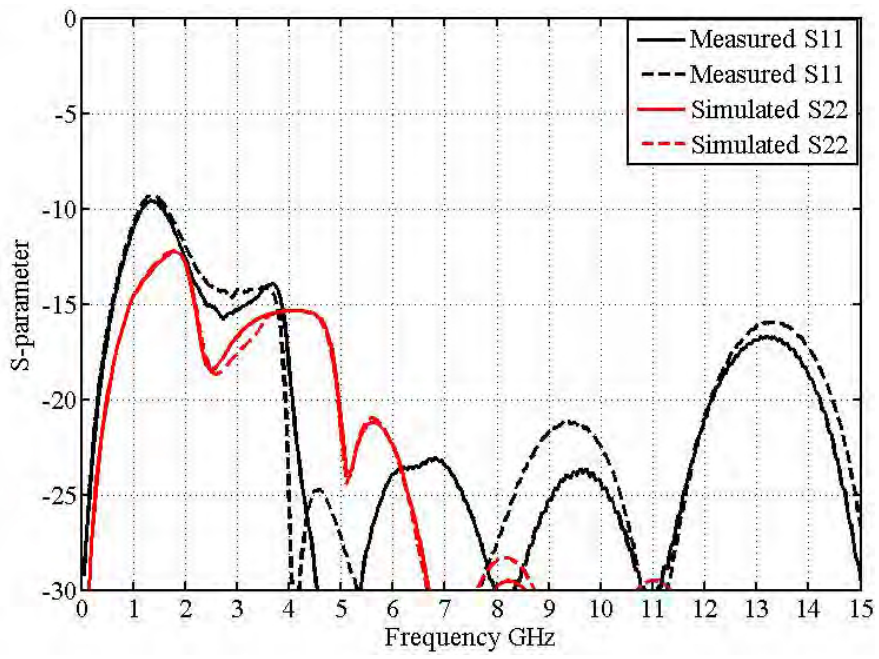

Figure 4.6b: Comparison of S11 and S22 between simulation and measurement when ferrite is biased transversely with internal magnetic field $H_{i}=660 \mathrm{Oe}$

\subsubsection{Prototype of a tunable attenuator}

Since the demagnetization factors could be ignored when the device is transversely biased, so, according to Kittel's equation, the resonance frequency could be found by 
Equation (4.1), where $H_{a}$ is the applied dc field.

$$
f_{r}=\frac{\mu_{0} \gamma}{2 \pi} \sqrt{H_{a}\left(H_{a}+M_{s}\right)}
$$

Figure 4.7 shows that the measured resonance frequency depends on the applied field. We then compare the measured resonance frequencies with the calculated ones, as shown in Figure 4.8. Although there is a difference of $1.5 \mathrm{GHz}$ as we explained before, generally they are in agreement.

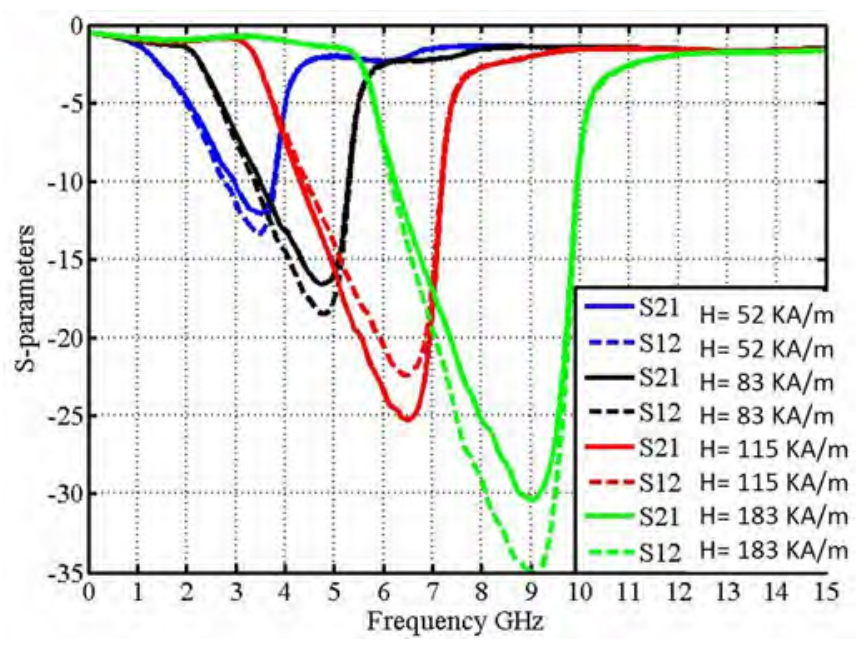

Figure 4.7: Measured resonance frequencies under different bias fields

Based on the theory and the measurements, a prototype of a tunable attenuator could be achieved by varying the transversal bias field. Considering that the ferrite LTCC technology allows 3D design, several windings in series (see Figure 4.9) could be integrated into the $\mathrm{CPW}$ structure to provide the internal bias field (without demagnetization field). This design could achieve a multi-functional attenuator: 1 . the attenuation frequency could be tuned by changing the dc current which varies the internal magnetization $\mathrm{M} ; 2$. the attenuation level could also be controlled by choosing the number of energized windings. 


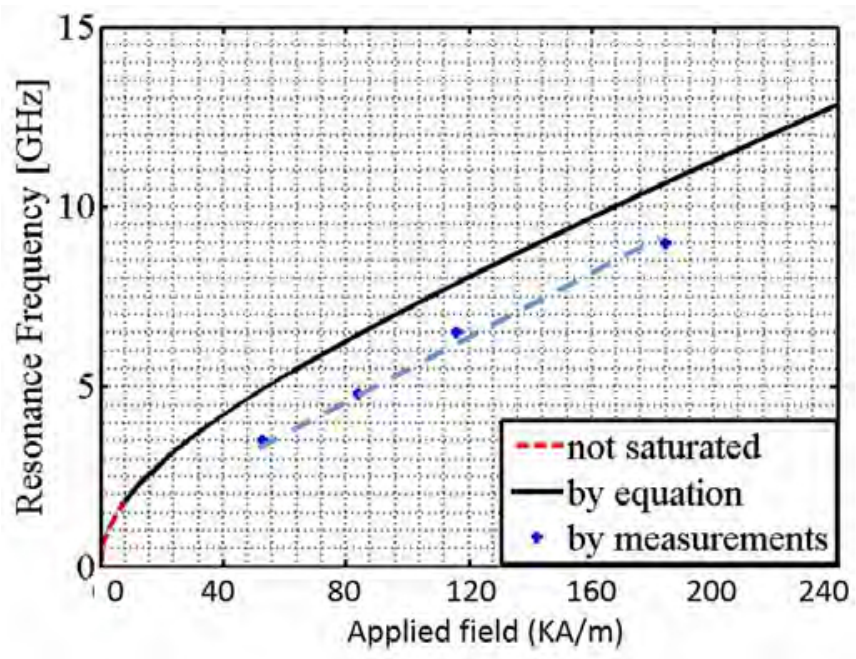

Figure 4.8: Comparison of measured and calculated resonance frequencies under different bias fields

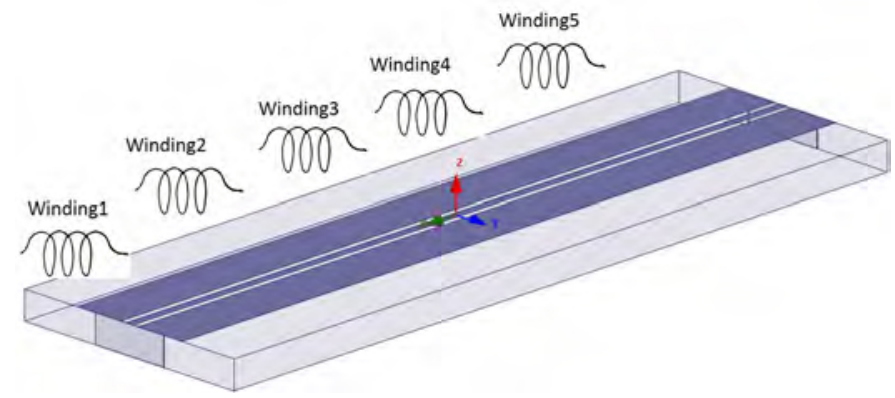

Figure 4.9: Prototype of a tunable attenuator with windings in series

This design is not in the scope of the thesis. However, the simulations and measurements of the ferrite LTCC CPW transmission line allow us to have a first experience in designing ferrite LTCC device.

\subsection{Different structures of circulators}

After having designed and measured the CPW based on ferrite LTCC, we will present some structures of circulators in theories, especially the stripline structure which is used in our final designs. 


\subsubsection{Stripline circulator}

The conventional stripline circulator is shown in Figure 4.10 [5]. It consists of two metal grounds, a center conductor and two ferrite disks. This is a symmetrical structure. Two ferrite discs are separated by the center conductor and three access transmission lines are spaced $120^{\circ}$ from each other. Two ground planes then envelop the structure. The space surrounding the ferrite discs is occupied by dielectric materials. The ferrite material is magnetized perpendicularly to the plane of the disk by an external static magnetic field created by two magnets located on either side of the structure.

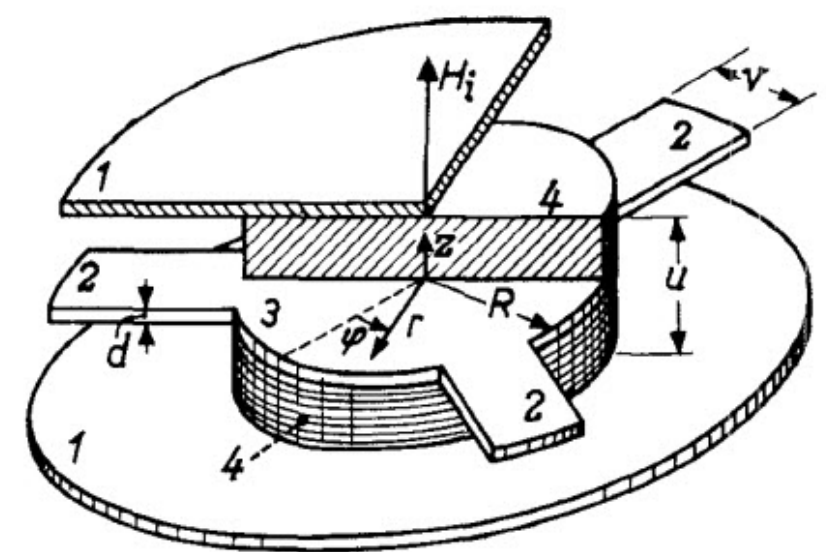

Figure 4.10: Principle construction of a stripline junction circulator: (1) two metal grounds; (2,3) center conductor; (4) ferrite disk; (5) V is the coupling angle; (6) $\mathrm{u}$ is the height of the ferrite disk; (7) $H_{i}$ is the bias field; (8) $\mathrm{d}$ is the thickness of conductors; (9) $\mathrm{R}$ radius of the center conductor [5]

The possibility of a three-port circulator was firstly postulated by Carlin [100] in 1954 based on the scattering-matrix analysis of the three-port microwave junction. The performance of an early experimental circulator (using waveguide) was described by Chait and Curry [101] in 1959. Since then, various types of circulators: stripline circulators [102-105] , three-port ring circulators [106] and other types [107] have 
been developed. The operating bands extend from the microwave to the millimeter range [108].

A stripline structure was chosen in this thesis because the two broad metal ground planes allow the bias windings to be placed immediately above and below the circulator core without coupling to the microwave fields. Hence the ground planes provide rf shielding while simultaneously allowing the magnetostatic bias field into the core.

\subsubsection{Microstrip circulator}

Microstrip line was firstly developed in ITT laboratories [109] and was a competitor of stripline. Now the microstrip line is one of the most popular types of planar transmission lines, primarily because it can be fabricated by photo-lithographic process and easily integrated with other passive and active microwave devices. These make microstrip circulators preferable in some circuit designs because it would provide an ideal compact circuit, compared to (stripline) triplate circulators or waveguide circulators. Conventionally, a Y-junction microstrip circulator consists of a dielectric substrate and a ferrite material which is put on it. A metal ground is located below the dielectric material. Finally, the center conductor of the circulator is designed on the top of the structure (see Figure 4.11 [17]). In some cases, the ferrite disk could also be placed above the center conductor.

The first reported microstrip circulator operating at $8.3 \mathrm{GHz}$ was realized by Hershenov, B. in 1966 [110]. Then another 3-port microstrip circulator was built in 1971 which operates near $30 \mathrm{GHz}$ with an isolation greater than $20 \mathrm{~dB}$ and a fractional bandwidth about $6 \%$ [111]. Recently, researchers are able to design microstrip circulators using LTCC technology [24]. Besides, thin ferrite film is also used to design a microstrip circulator [112]. Moreover, by depositing barium ferrite films on sapphire 


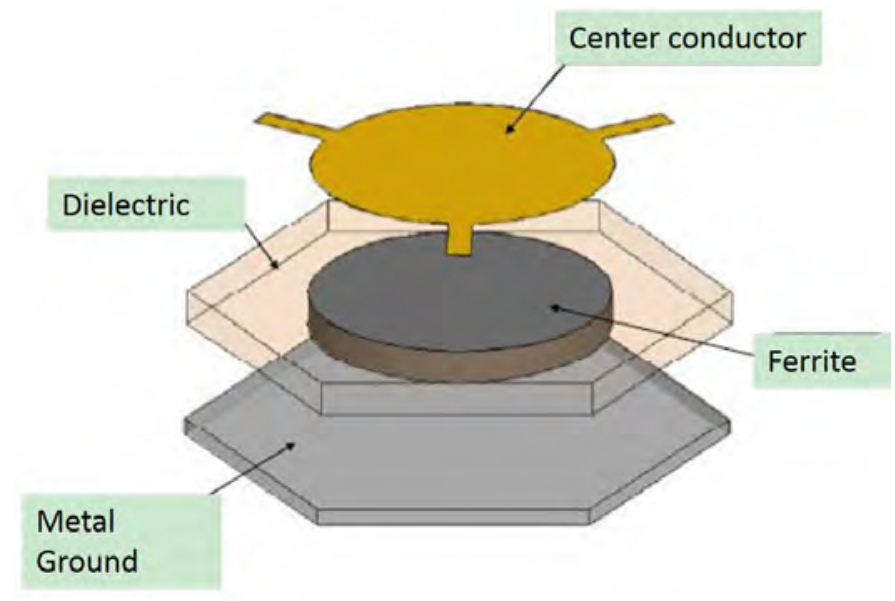

Figure 4.11: Principle construction of a microstrip junction circulator [17]

substrate, a self-biased microstrip junction circulator at $26 \mathrm{GHz}$ was realized [113] in 2011 which allows the integration of ferrite passive nonreciprocal components with monolithic microwave integrated-circuit (MMIC) devices.

\subsubsection{Coplanar circulator}

A circulator with coplanar waveguide (CPW) structure is also a good candidate for microwave integrated circuits because the transmission line (signal) and the metal grounds (GND) are located in the same plane (usually on the top). Thus, coplanar circulators can be easily fabricated at a low cost by using lithography process. Figure 4.12 [18] shows a typical coplanar structure of a hexagonal circulator with CPW structure.

Wen and Bayard have firstly reported coplanar circulators operating at $7 \mathrm{GHz}$ which need an internal magnetic field greater than $170 \mathrm{kA} / \mathrm{m}$ [114,115]. Then Ogasawara reported that Y-junction circulators with a CPW could successfully operate at different frequencies according to different external de magnetic fields applied perpendicularly to the ferrite surface [116]. Recently a CPW circulator including BaM 


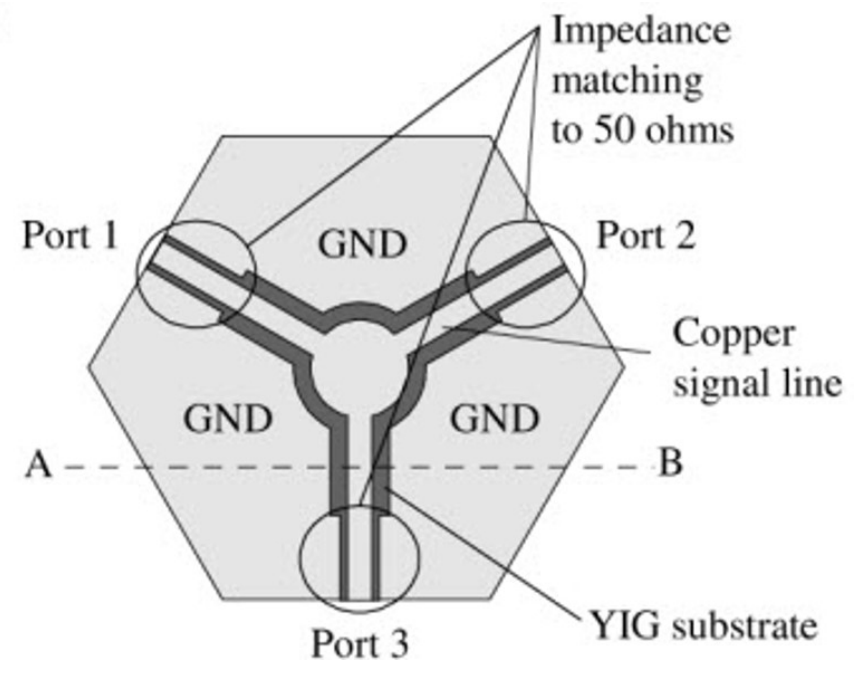

Figure 4.12: Top view of the signal line and the ground plane of a hexagonal circulator with CPW structure [18]

(Barium ferrite) thin films and dielectric layer with a low dielectric constant is also proposed and simulated in [117]. In 2011, a ferrite was formed by embedding magnetic particles into a host dielectric matrix in laboratory LT2C and a coplanar circulator was fabricated based on it [118].

\subsection{Theory of stripline junction circulators}

As presented before, the design of a stripline Y-junction circulator (see Figure 4.13) is generally based on the work of Bosma [5] who solves the simplified boundary-value problem of the circulator ferrite disk by using a Green function approach. Then Fay and Comstock [38] describe the operation of a circulator in terms of counterrotating propagation modes in the ferrite disk. Wu and Rosenbaum [39] predict octave bandwidth microstrip circulator operation theoretically using Bosma's Green function analysis. The Green function relates $E_{z}$ and $H_{\Phi}$ for lossless circulators when the effective permeability $\mu_{e f f}>0$. Assuming $e^{j w t}$ harmonic time dependence, 


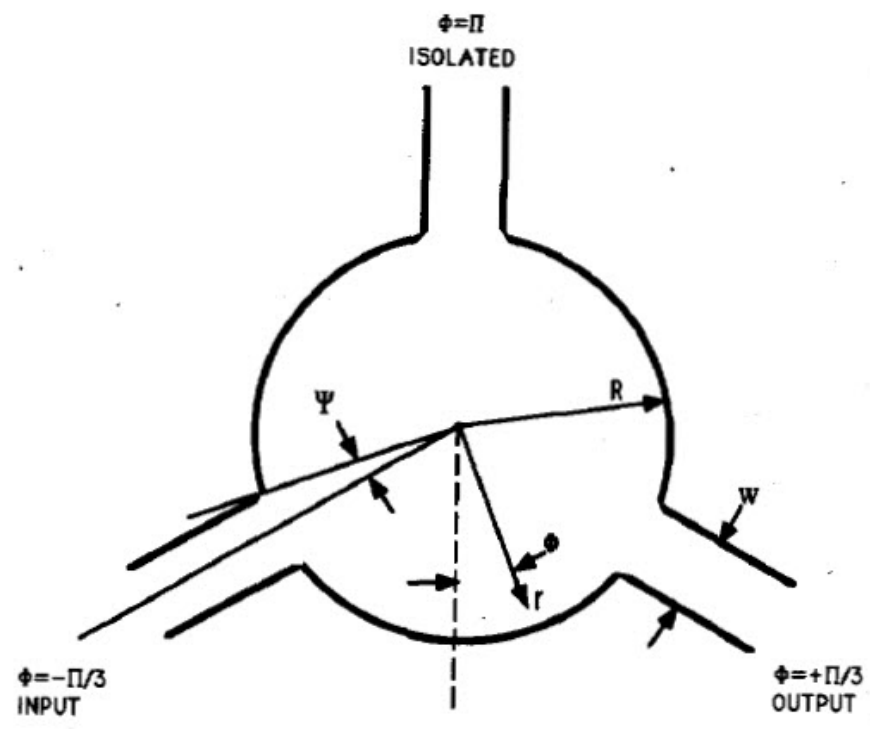

Figure 4.13: Circulator coordinate reference

Bosma's Green function has the following form (see Equation (4.2)):

$$
\begin{aligned}
G\left(r, \phi ; R, \phi^{\prime}\right)= & -\frac{j Z_{e f f} J_{0}(k r)}{2 \pi J_{0}^{\prime}(x)}+ \\
& \frac{Z_{e f f}}{\pi} \sum_{n=1}^{\infty} \frac{\frac{k}{\mu} \frac{n J_{n}(x)}{x} \sin n\left(\phi-\phi^{\prime}\right)-j J_{n}^{\prime}(x) \cos n\left(\phi-\phi^{\prime}\right)}{\left\{J_{n}^{\prime}(x)\right\}^{2}-\left\{\frac{k}{\mu} \frac{n J_{n}(x)}{x}\right\}^{2}} \times J_{n}(k r)
\end{aligned}
$$

where:

- $\kappa$ and $\mu$ : Polder tensor elements

- $J_{n}(x)$ : Bessel function of the first kind with order $\mathrm{n}$

- $x: \mathrm{x}=\mathrm{kR}$

- $k$ : the propagation constant $k=\omega \sqrt{\epsilon_{0} \mu_{0} \epsilon_{f} \mu_{e f f}}$

- $R$ : ferrite disk radius 
- $\omega$ : circulator's operating frequency

- $\epsilon_{0}$ : permittivity of free-space $\epsilon_{0}=8.854 \times 10^{-12}$

- $\epsilon_{f}$ : relative permittivity of ferrite

- $\epsilon_{d}$ : relative permittivity of dielectric around ferrite

- $\mu_{e f f}$ : effective permeability of ferrite $\mu_{e f f}=\frac{\mu^{2}-\kappa^{2}}{\mu}$

- $J_{n}^{\prime}(x)$ : derivative of Bessel function $J_{n}(x)$ with respect to its argument

- $Z_{d}$ : impedance of the dielectric material

- $Z_{\text {eff }}$ : intrinsic wave impedance of ferrite $Z_{\text {eff }}=\sqrt{\frac{\mu_{0} \mu_{e f f}}{\varepsilon_{0} \varepsilon_{f}}}$

The roots of $J_{n}^{\prime}(x)=0$ are shown in Equation (4.3), where $x_{n m}$ means that the $m^{\text {th }}$ order root of $J_{n}^{\prime}(x)=0$.

$$
\left\{\begin{array}{l}
x_{11}=1.841 \\
x_{21}=3.054 \\
x_{31}=4.201 \\
x_{12}=5.331
\end{array}\right.
$$

However, not all terms are important in practical applications and most circulators operate close to the $n=1$ resonance, so many people consider only the $n=1$ term leading to the formula of the radius $\mathrm{R}$ of the ferrite disk (Equation (4.4a)) and the coupling angle $\Phi$ (Equation (4.4b)), where $Z_{d}$ is the wave impedance of the dielectric around the ferrite disk $\left(Z_{d}=\frac{120 \pi}{\sqrt{\varepsilon_{d}}}\right)$.

$$
k R=x_{11}=1.841
$$




$$
\Phi=\frac{\pi}{\sqrt{3}(1.84)} \frac{Z_{d}}{Z_{e f f}}\left|\frac{\kappa}{\mu}\right|
$$

Since there are no radial currents at the edge of the ferrite disks except at the three access ports (neglecting fringing), Bosma, Davies and Cohen [119], and others suggest the following boundary condition for the three-port Y-junction circulator (Equation (4.5)):

$$
H_{\phi}(R, \Phi)= \begin{cases}H_{1}, & -\frac{\pi}{3}-\Psi<\phi<-\frac{\pi}{3}+\Psi \\ H_{2}, & \frac{\pi}{3}-\Psi<\phi<\frac{\pi}{3}+\Psi \\ H_{3}, & \pi-\Psi<\phi<\pi+\Psi \\ 0, & \text { elsewhere }\end{cases}
$$

Equation (4.6) is also employed according to the cyclic symmetry:

$$
G\left(\phi+\frac{2 \pi}{3} ; \phi^{\prime}+\frac{2 \pi}{3}\right)=G\left(\phi ; \phi^{\prime}\right)
$$

Since the circulator is symmetrical, the impedance matrix is therefore defined by Equation (4.7).

$$
[\eta]=\left[\begin{array}{ccc}
\eta_{11} & \eta_{31} & \eta_{21} \\
\eta_{21} & \eta_{11} & \eta_{31} \\
\eta_{31} & \eta_{21} & \eta_{11}
\end{array}\right]
$$

The electric field on any port at the observation point due to a distribution of unit point source is given by the superposition integral (Equation (4.8)) [63]:

$$
E_{z}(R, \phi)=\int_{c} G\left(\phi \mid \phi^{\prime}\right) H_{\phi}\left(R, \phi^{\prime}\right) d \phi^{\prime}
$$


Then the impedance at each port could be derived (Equation (4.9)) because the magnetic field $H_{\Phi}$ is constant at each port according to the boundary condition:

$$
\eta=\frac{E_{z}}{H_{\phi}}=\int_{c} G\left(\phi \mid \phi_{0}\right) d \phi
$$

The observation point is assumed to be $\phi_{0}=-\pi / 3$ in this calculation, then the wave impedance at port 1 is (Equations (4.10a) and (4.10b)):

$$
\begin{aligned}
& \eta_{11}=\int G\left(\phi \mid-\frac{\pi}{3}\right) d \phi \quad-\frac{\pi}{3}-\Psi<\phi<-\frac{\pi}{3}+\Psi \\
& \eta_{11}=-\int_{-\frac{\pi}{3}-\Psi}^{-\frac{\pi}{3}+\Psi} \frac{j Z_{\text {eff }} J_{0}(k r)}{2 \pi J_{0}^{\prime}(x)} d \phi+ \\
& \int_{-\frac{\pi}{3}-\Psi}^{-\frac{\pi}{3}+\Psi} \frac{Z_{\text {eff }}}{\pi} \sum_{n=1}^{\infty} \frac{\frac{k}{\mu} \frac{n J_{n}(x)}{x} \sin n\left(\phi+\frac{\pi}{3}\right)-j J_{n}^{\prime}(x) \cos n\left(\phi+\frac{\pi}{3}\right)}{\left\{J_{n}^{\prime}(x)\right\}^{2}-\left\{\frac{k}{\mu} \frac{n J_{n}(x)}{x}\right\}^{2}} \times J_{n}(k r) d \phi \\
& =-\frac{j Z_{e f f} J_{0}(k r)}{2 \pi J_{0}^{\prime}(x)} \times(2 \Psi)+ \\
& \frac{Z_{\text {eff }}}{\pi} \sum_{n=1}^{\infty} \frac{-j 2 J_{n}^{\prime}(x) \Psi \operatorname{sinc}(n \Phi)}{\left\{J_{n}^{\prime}(x)\right\}^{2}-\left\{\frac{k}{\mu} \frac{n J_{n}(x)}{x}\right\}^{2}} \times J_{n}(k r)
\end{aligned}
$$

where $\operatorname{sinc}(n \Psi)=\frac{\sin (n \Psi)}{n \Psi}$

Similarly, the impedance at port 2 and port 3 can be derived in Equation (4.11b) and Equation (4.12b), respectively.

$$
\eta_{21}=\int G\left(\phi \mid-\frac{\pi}{3}\right) d \phi \quad \frac{\pi}{3}-\Psi<\phi<\frac{\pi}{3}+\Psi
$$




$$
\begin{aligned}
& \eta_{21}=-\int_{\frac{\pi}{3}-\Psi}^{\frac{\pi}{3}+\Psi} \frac{j Z_{\text {eff }} J_{0}(k r)}{2 \pi J_{0}^{\prime}(x)} d \phi+ \\
& \int_{\frac{\pi}{3}-\Psi}^{\frac{\pi}{3}+\Psi} \frac{Z_{e f f}}{\pi} \sum_{n=1}^{\infty} \frac{\frac{k}{\mu} \frac{n J_{n}(x)}{x} \sin n\left(\phi+\frac{\pi}{3}\right)-j J_{n}^{\prime}(x) \cos n\left(\phi+\frac{\pi}{3}\right)}{\left\{J_{n}^{\prime}(x)\right\}^{2}-\left\{\frac{k}{\mu} \frac{n J_{n}(x)}{x}\right\}^{2}} \times J_{n}(k r) d \phi \\
& =-\frac{j Z_{e f f} J_{0}(k r)}{2 \pi J_{0}^{\prime}(x)} \times(2 \Psi)+ \\
& \frac{Z_{e f f}}{\pi} \sum_{n=1}^{\infty} \frac{2 \Psi \operatorname{sinc}(n \Phi)\left[\frac{\kappa}{\mu} \frac{n J_{n}(x)}{x} \sin \frac{2 n \pi}{3}-i J_{n}^{\prime}(x) \cos \frac{2 n \pi}{3}\right]}{\left\{J_{n}^{\prime}(x)\right\}^{2}-\left\{\frac{k}{\mu} \frac{n J_{n}(x)}{x}\right\}^{2}} \times J_{n}(k r) \\
& \eta_{31}=\int G\left(\phi \mid-\frac{\pi}{3}\right) d \phi \quad \pi-\Psi<\phi<\pi+\Psi \\
& \eta_{31}=-\int_{\pi-\Psi}^{\pi+\Psi} \frac{j Z_{\text {eff }} J_{0}(k r)}{2 \pi J_{0}^{\prime}(x)} d \phi+ \\
& \int_{\pi-\Psi}^{\pi+\Psi} \frac{Z_{e f f}}{\pi} \sum_{n=1}^{\infty} \frac{\frac{k}{\mu} \frac{n J_{n}(x)}{x} \sin n\left(\phi+\frac{\pi}{3}\right)-j J_{n}^{\prime}(x) \cos n\left(\phi+\frac{\pi}{3}\right)}{\left\{J_{n}^{\prime}(x)\right\}^{2}-\left\{\frac{k}{\mu} \frac{n J_{n}(x)}{x}\right\}^{2}} \times J_{n}(k r) d \phi \\
& =-\frac{j Z_{e f f} J_{0}(k r)}{2 \pi J_{0}^{\prime}(x)} \times(2 \Psi)+ \\
& \frac{Z_{e f f}}{\pi} \sum_{n=1}^{\infty} \frac{2 \Psi \operatorname{sinc}(n \Phi)\left[\frac{\kappa}{\mu} \frac{n J_{n}(x)}{x} \sin \frac{4 n \pi}{3}-i J_{n}^{\prime}(x) \cos \frac{4 n \pi}{3}\right]}{\left\{J_{n}^{\prime}(x)\right\}^{2}-\left\{\frac{k}{\mu} \frac{n J_{n}(x)}{x}\right\}^{2}} \times J_{n}(k r)
\end{aligned}
$$

Now, we have all elements of the impedance matrix (Equation (4.7)). We then normalize the impedance $[\eta]$ by the dielectric impedance $Z_{d}$ leading to $[\mathrm{Z}]=[\eta] / Z_{d}$. The next step is to transform the matrix $[\mathrm{Z}]$ to matrix $[\mathrm{S}]$, by $[S]=[Z+I]^{-1}[Z+I]$, where [I] is identity matrix. Then the elements of [S] could be obtained by Equations (4.13a) 
to $(4.13 \mathrm{c})$.

$$
\begin{gathered}
S_{11}=1+\frac{-2 Z_{d}\left[\left(Z_{11}^{2}+1\right)-Z_{21} Z_{31}\right]}{\left(Z_{11}+1\right)^{3}+Z_{21}^{3}+Z_{31}^{3}-3 Z_{21} Z_{31}\left(Z_{11}+1\right)} \\
S_{21}=\frac{-2 Z_{d}\left[Z_{31}^{2}-Z_{21}\left(Z_{11}+1\right)\right]}{\left(Z_{11}+1\right)^{3}+Z_{21}^{3}+Z_{31}^{3}-3 Z_{21} Z_{31}\left(Z_{11}+1\right)} \\
S_{31}=\frac{-2 Z_{d}\left[Z_{21}^{2}-Z_{31}\left(Z_{11}+1\right)\right]}{\left(Z_{11}+1\right)^{3}+Z_{21}^{3}+Z_{31}^{3}-3 Z_{21} Z_{31}\left(Z_{11}+1\right)}
\end{gathered}
$$

To simplify the expression of these S-parameters, three variables are defined by the Equations (4.14a) to (4.14c):

$$
\begin{aligned}
C_{1} & =j \pi \frac{Z_{11}+1}{2 Z_{\text {eff }}} \\
C_{2} & =j \pi \frac{Z_{31}}{2 Z_{\text {eff }}} \\
C_{2} & =j \pi \frac{Z_{21}}{2 Z_{\text {eff }}}
\end{aligned}
$$

Hence the elements of [S] (Equations (4.13a) to (4.13c)) can be rewritten in Equations (4.15a), (4.15c) and (4.15c)

$$
\begin{gathered}
S_{11}=1+\frac{\pi Z_{d}}{j Z_{\text {eff }}} \frac{C_{1}^{2}-C_{2} C_{3}}{C_{1}^{3}+C_{2}^{3}+C_{3}^{3}-3 C_{1} C_{2} C_{3}} \\
S_{21}=\frac{\pi Z_{d}}{j Z_{\text {eff }}} \frac{C_{2}^{2}-C_{1} C_{3}}{C_{1}^{3}+C_{2}^{3}+C_{3}^{3}-3 C_{1} C_{2} C_{3}} \\
S_{31}=\frac{\pi Z_{d}}{j Z_{\text {eff }}} \frac{C_{3}^{2}-C_{1} C_{2}}{C_{1}^{3}+C_{2}^{3}+C_{3}^{3}-3 C_{1} C_{2} C_{3}}
\end{gathered}
$$

Finally we can get the matrix [S] (see Equation (4.16)) which is in agreement with that in article [120]. For more details of these calculations, please refer to the Appendix A. 


$$
[S]=\left[\begin{array}{lll}
S_{11} & S_{31} & S_{21} \\
S_{21} & S_{11} & S_{31} \\
S_{31} & S_{21} & S_{11}
\end{array}\right]
$$

A Matlab program is realized based on the above calculations which allows to simulate a circulator mathematically. This is very useful in designing circulators, because this program could give us approximate values of some important parameters such as the radius of ferrite disk, operating frequency, coupling angle, bias field etc, which could be used in the optimization by the EM simulator.

\subsection{Ferrite LTCC circulator with external magnet}

In order to verify the feasibility of a circulator based on ferrite LTCC technology, a stripline circulator with external magnets is firstly designed, fabricated and measured.

\subsubsection{Theory and design}

Since the entire volume in this design is ferrite LTCC material, the magnetic wall in Bosma's theory cannot be well defined, so the edge-guided mode structure is chosen. As said before, the edge-guided mode circulator has a potential broadband performance [59] [60]. The edge-guided mode is based on Hine's studies of the edgeguided-wave (EGW) propagation in microwave integrated circuits. The effect of field displacement concentrates the electromagnetic (EM) field on one edge of the stripline for one propagation direction and on the other for the reverse direction.

Although the circulator designed here is an edge-guided mode circulator, the theory of Bosma based on the volume resonance is still useful to predict approximately 
the circulator's performance. The analytical Matlab program mentioned in the previous section is employed for this purpose. This program contains two parts: one part to calculate the Polder tensor $\left(\mu, \kappa\right.$ and $\left.\mu_{z}\right)$; the other part to calculate Z-parameters and then convert them to S-parameters. As explained, the theory of this calculation has been already presented in the following references [39] [120]. The inputs of this Matlab program are the basic parameters concerning the materials and circulator, such as: relative permittivity of the dielectric $\varepsilon_{d}$, relative permittivity of the ferrite $\varepsilon_{f}$, magnetic saturation of the ferrite $M_{s}$, internal $\mathrm{H}$ field $H_{i}$, damping factor $\alpha$, circulator's operating frequency $f_{c}$, number of modes to be taken into account $\mathrm{N}$, frequency range and step, etc

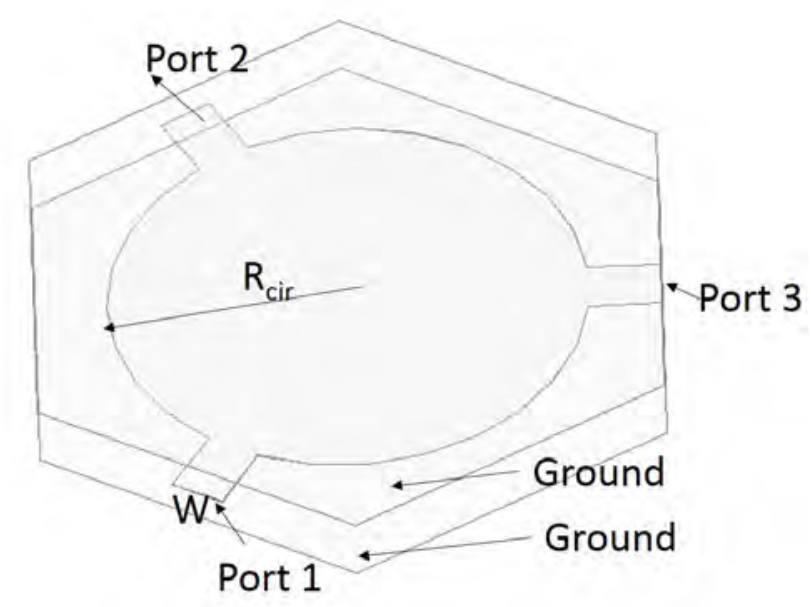

Figure 4.14: Stripline circulator configuration

With these parameters, the Matlab program is able to calculate the circulator's width $\mathrm{W}$ and the radius $R_{\text {cir }}$ (see Figure 4.14) and then S-parameters could be shown with three curves in dB: transmission S31, isolation S21 and reflection S11. Figure 4.15 indicates a possible circulation in Matlab at $15 \mathrm{GHz}$ with $R_{\text {cir }}=3 \mathrm{~mm}$ and $\mathrm{W}=0.4 \mathrm{~mm}$. If the approximation based on the Bosma's theory (Equations (4.4a) and (4.4b) [39]) is not good, the width $\mathrm{W}$ and $R_{\text {cir }}$ could also be entered manually, 
which allows us to apply an improved approximation.

The problem of this mathematical approximation is that the height of the model cannot be considered, so an EM simulator is still necessary to finalize the design by optimizing the previous parameters found by the analytical program.

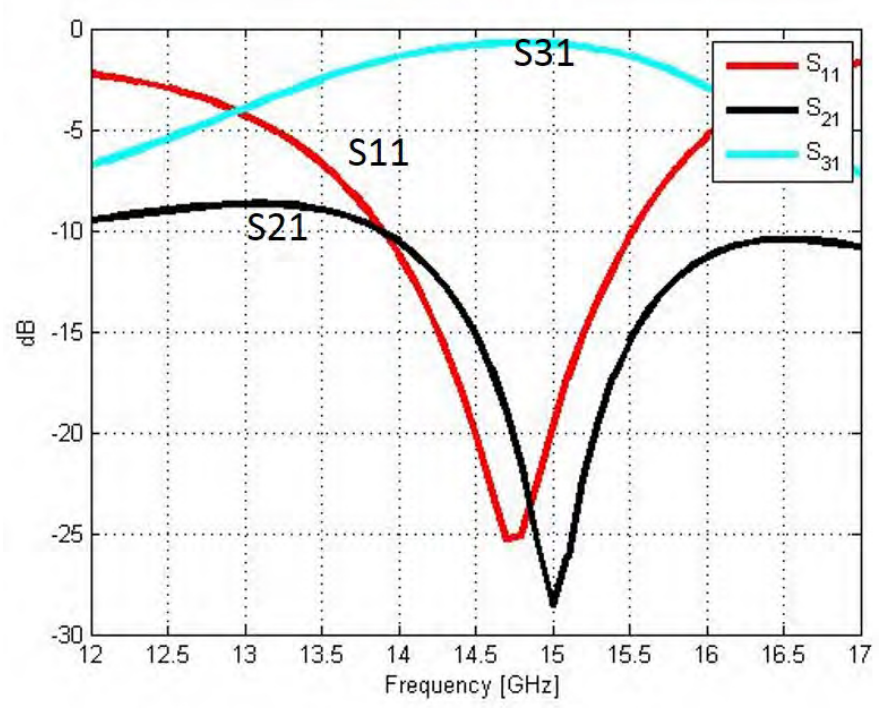

Figure 4.15: Calculated S-parameters in Matlab

An edge-guided circulator is then designed in Ansys HFSS. The theories about edge-guided circulators with three ports are presented in $[59,60]$. The key parameters are the radius $\mathrm{R}$ which gives the curvature (see Figure 4.16) and the width $\mathrm{W}$ which is the open width of the center conductor. These parameters must be determined according to the operating frequency and the material's properties. Furthermore, the two metal grounds are placed on the layer above the center conductor and the layer below. By optimizing the $\mathrm{W}, \mathrm{R}$ and $R_{\text {cir }}$, the operating frequency is fixed to $15 \mathrm{GHz}$ to avoid low-field losses. The next difficulty consists in the transition from $\mathrm{CPW}$ (on the top layer) to stripline (circulator). Three CPW transmission lines are designed on the top layer and the lower ground plane of the circulator is designed to have three hands to connect the vias from the top layer (see Figure 4.17). Then, 
each ground of one CPW transmission line is connected to both ground planes of the circulator by two vias: one goes down to the upper ground, the other goes to the lower one, finally the CPW line's signal conductor is connected to the circulator's center conductor. The reason why all the metal grounds are connected is to avoid exciting a parallel-plate mode.

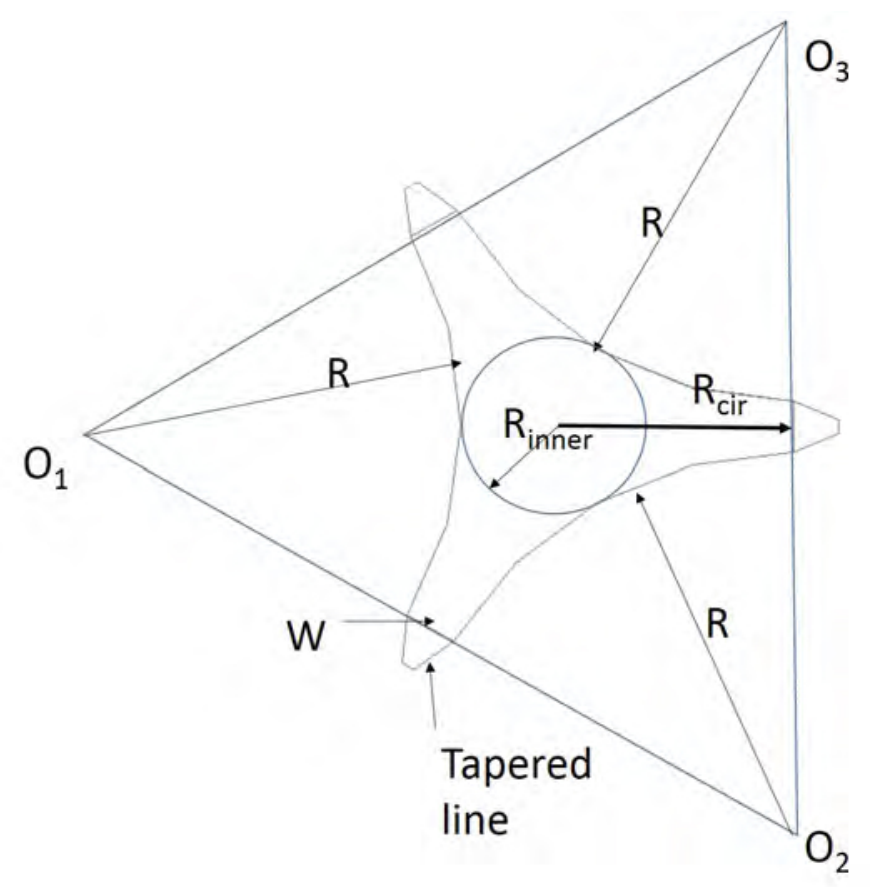

Figure 4.16: Parameters of an edge-guided circulator

The optimization is performed using Ansoft HFSS software and leads to the final dimensions $R_{\text {cir }}=2.8 \mathrm{~mm}, \mathrm{~W}=0.38 \mathrm{~mm}$ (see Figure 4.17), the magnetic-biased area has a diameter of $1 \mathrm{~mm}$ and the internal $\mathrm{H}$-field is close to $120 \mathrm{kA} / \mathrm{m}$ which is much smaller than the one given in reference [24]. The simulation result, shown in Figure 4.18, meets that of Matlab: transmission $-0.84 \mathrm{~dB}$, isolation $-29 \mathrm{~dB}$ and the refection approximately $-19 \mathrm{~dB}$. The operating frequency is $14 \mathrm{GHz}$ from HFSS instead of $15 \mathrm{GHz}$ predicted by the analytical program. This is probably due to: 1 . the Matlab program performs only a 2D mathematical analysis; 2 . The 3D model in 
HFSS is an edge-guide mode circulator. One CPW transmission line is also fabricated to serve the network analyzer calibration.

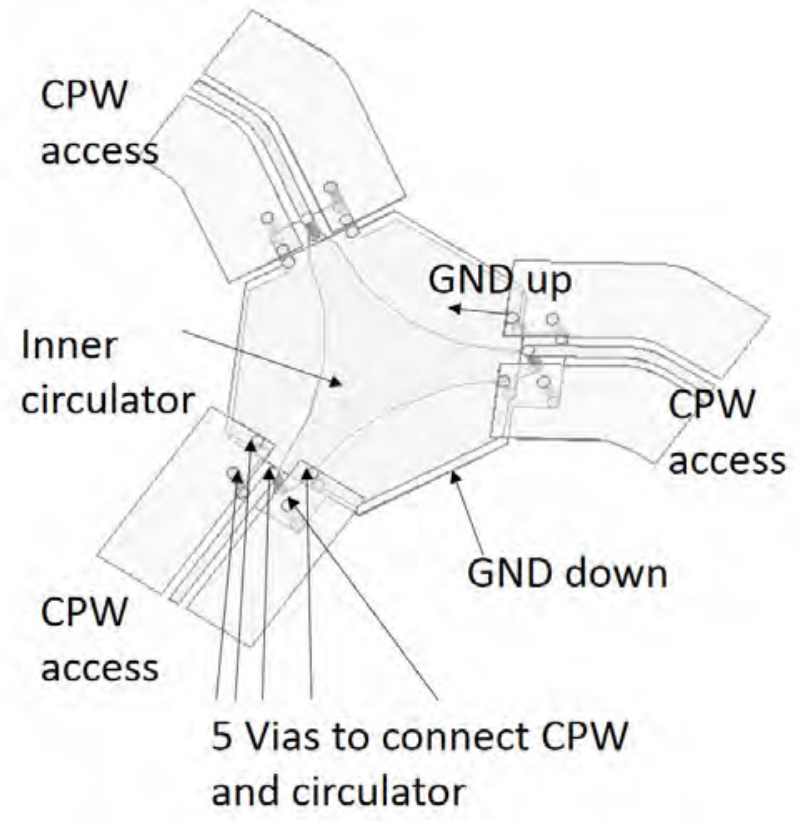

Figure 4.17: Edge-guided circulator's 3D design

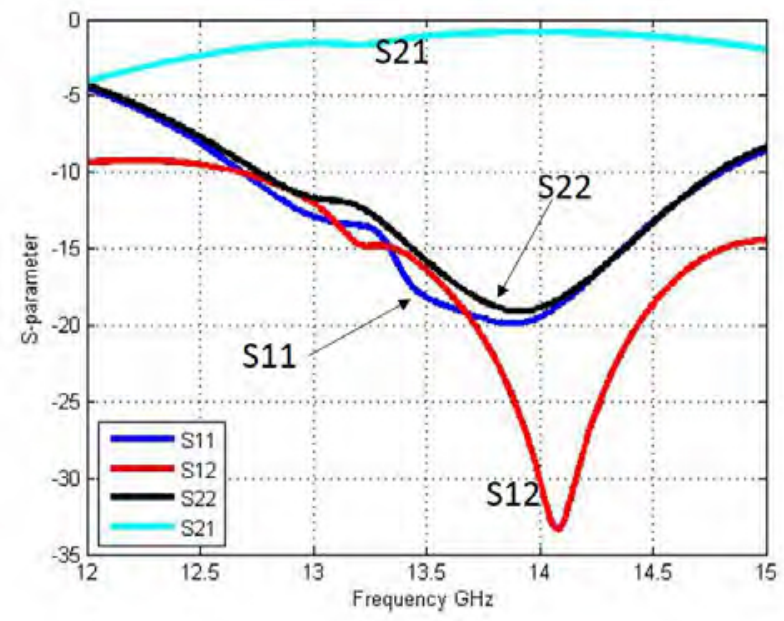

Figure 4.18: Simulated S-parameters in HFSS when port 3 is matched: a circulator working at $15 \mathrm{GHz}$ 


\subsubsection{Measurement}

The LTCC edge-guided circulator is then fabricated. For the probe station, the angle between two adjacent ports is $90^{\circ}$, so two of the CPW feed lines contain small bends to accommodate the probe station and care is taken to ensure that these bends do not cause reflections at $15 \mathrm{GHz}$. The measurement of the CPW line is shown in Figure 4.19, which shows that the performance of the unbiased LTCC material is good between $10 \mathrm{GHz}$ and $17 \mathrm{GHz}$. As predicted [121], the attenuation is indeed strong below $10 \mathrm{GHz}$ due to the low-field losses as explained in the previous chapter.

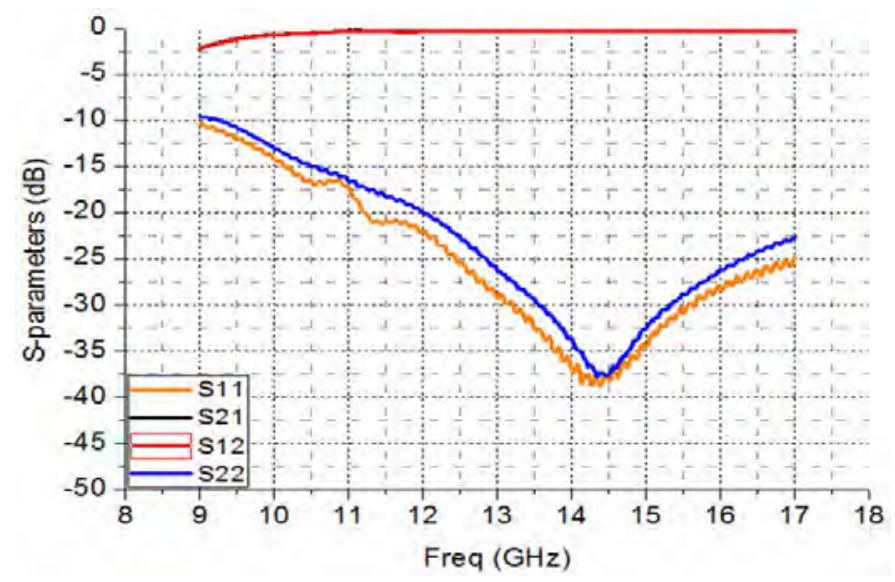

Figure 4.19: The measurement of the CPW verifies the calibration and demonstrates that the loss below $10 \mathrm{GHz}$ is strong

We connect the port 3 of the circulator to a matched load and then the circulator is measured. The internal magnetic field bias is obtained by applying a carefully chosen external magnet with a surface field 5233 Gauss on the top layer of the substrate. The bias magnetized area diameter is $1.59 \mathrm{~mm}$ which is larger than the diameter $(1 \mathrm{~mm})$ predicted by HFSS. The measurement of the circulators is performed with a two-port

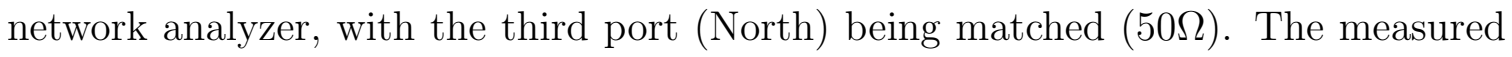
transmission and isolation is approximately $-1.8 \mathrm{~dB}$ and $-20 \mathrm{~dB}$ respectively as shown in Figure 4.20. The measurement also shows that the operating frequency is about 
$15 \mathrm{GHz}$ and at this center frequency, the return loss is better than $20 \mathrm{~dB}$. An internal resonance is also observed from the transmission parameter (S21) at approximately 15.5 GHz. This is perhaps due to the placement of the metallized magnet on the top layer coupling with the rf field. Since the whole volume is ferrite material, the magnets could probably only saturate the center leading to other parts partially magnetized, which is likely the cause of the higher than expected insertion loss. Although a small magnet is desired in this work, the insertion loss could be improved by using a larger and more powerful magnet.

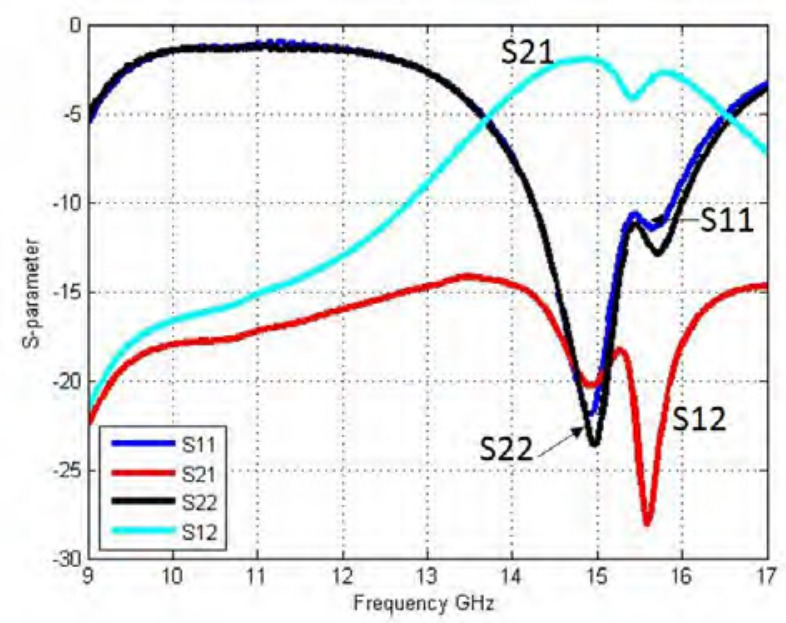

Figure 4.20: The measurement of the circulator shows the working frequency is 15 $\mathrm{GHz}$

\subsection{Ferrite LTCC circulator with integrated wind- ing}

The above design demonstrates that circulators could be designed on the ferrite LTCC substrate. In order to remove the bulky external magnet and avoid the demagnetization factor, in this section, a circulator with an integrated winding using the same 
ferrite LTCC tape will be presented.

\subsubsection{Bias winding design}

It would be best if the winding could provide a uniform and perpendicular (zdirection) magnetostatic bias field in the circulator core, generating a wide range of magnetization value in the ferrite LTCC. The hexagonal planar coil is chosen for this purpose, because it approaches a circular coil which has the maximum area for a given radius.

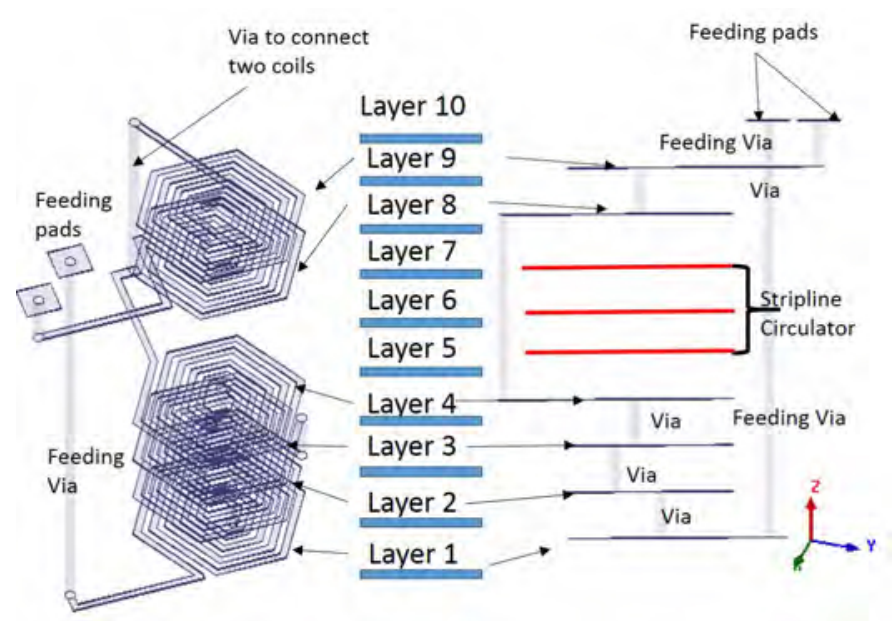

Figure 4.21a: The dc winding consists of 4 coils below the circulator and 2 coils above it

As shown in Figure 4.21a, the space priority is given to the winding. Stripline circulator only occupies three layers (5-7) and the top layer is reserved for connections, therefore, all the other layers are used to design the winding. The winding consists of four coils below the circulator on layer 1-4 and two coils above the circulator on layer 8 and 9. The 2D coil design is shown in Figure 4.21b. Each coil consists of 5 turns of a 0.1-mm wide conductor with a $0.1-\mathrm{mm}$ spacing: both are the minimum values allowed in the fabrication process. The inner coil turn has a side-to-side width of 0.4 
$\mathrm{mm}$ whereas the outer turn has a side-to-side width of $2.2 \mathrm{~mm}$. The reason why we don't use the conception which has three coils below the circulator and three coils above the circulator is that the circulator's metal ground will block the interconnection via between the upper coils and lower coils. Therefore the number of the coils should be even in our case.

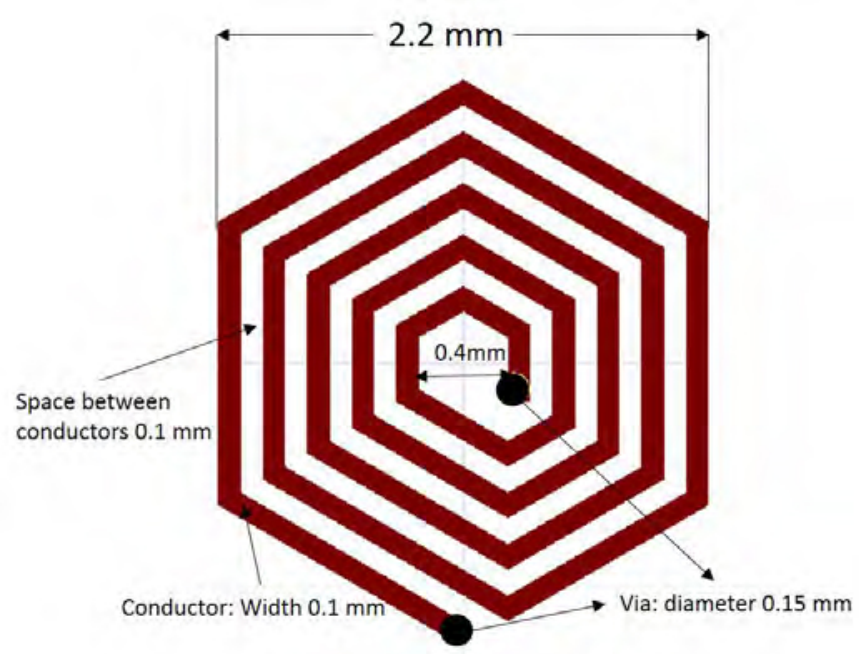

Figure 4.21b: Hexagonal coil dimensions

Current in the winding is supplied by two top-layer dc pads and $150-\mu \mathrm{m}$ diameter vias are used to connect the coils as shown in Figure 4.21a. The current returns to the top layer from the bottom layer through a stacked via that is placed beside the hexagonal ground planes of the circulator. Figure 4.21c demonstrates the desired 2-D conceptual sketch of the biasing magnetic flux.

\section{Magnetostatic simulation by Ansys Maxwell 3D}

The magnetostatic simulation is performed with Ansys Maxwell 3D by putting the winding into a block of ferrite LTCC that has the same dimensions as the anticipated diced prototypes. The B-H curve of the ESL 40012 material is used for this simulation 


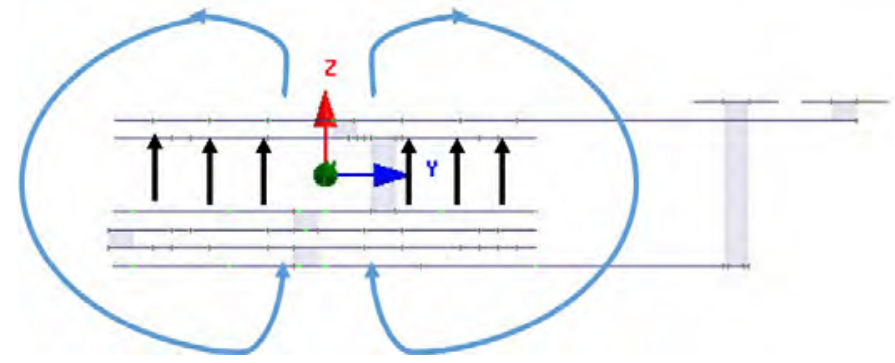

Figure 4.21c: 2D conceptual sketch of the biasing magnetic flux

[27]. Figure 4.22a shows the strength of the internal magnetic field $B_{i}$ along the z-axis in the middle of the winding (the dash cut line).

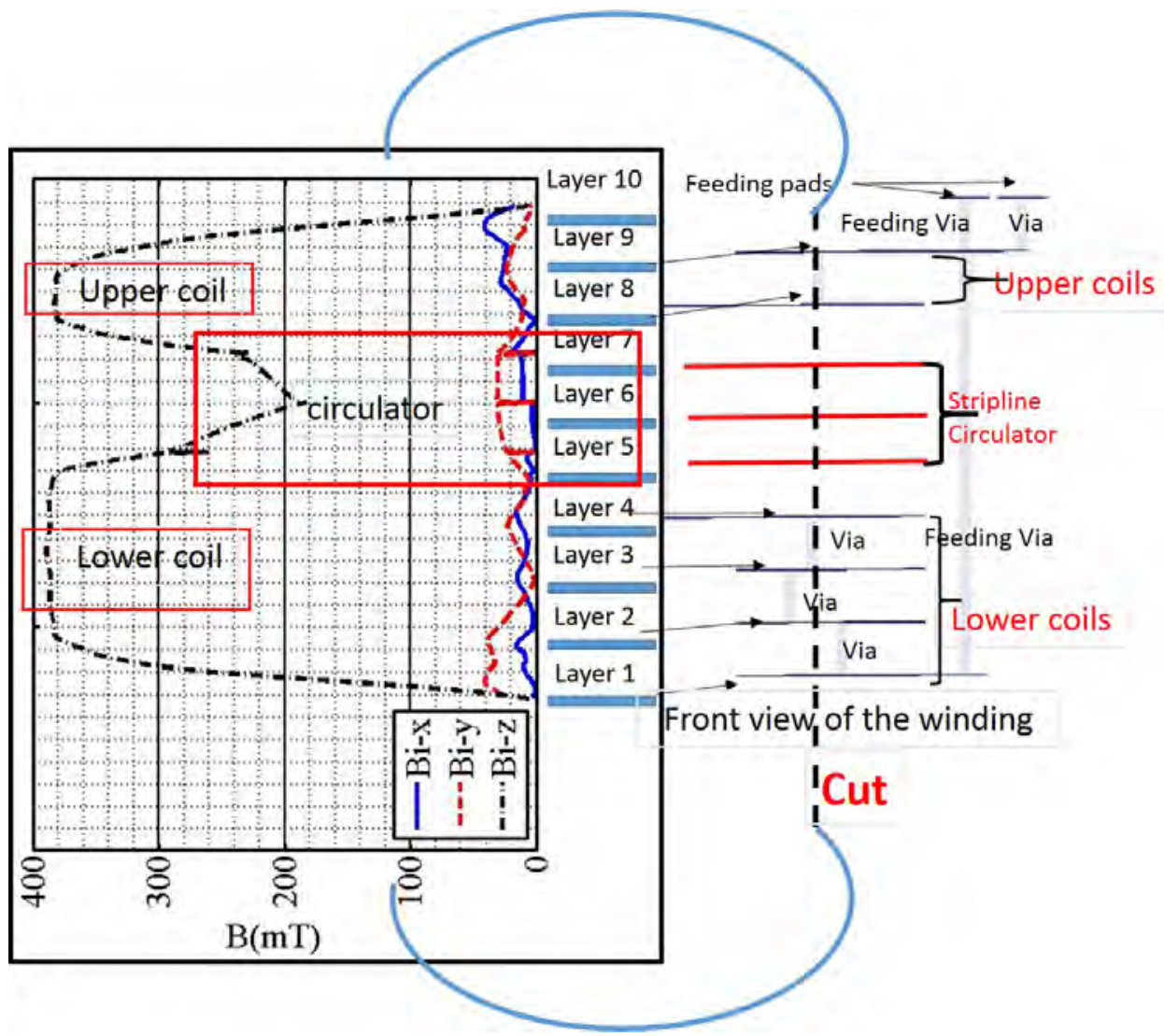

Figure 4.22a: Magneto-static simulation of winding in ferrite LTCC with a B-H curve: $\mathrm{Bi}$ along $\mathrm{Z}$ axis

The simulated dc magnetic field mainly follows the direction of z-axis which meets our expectation. Although high at the centre of the upper and lower coils, the $\mathrm{Z}$ 
component of the internal field is only approximately $200 \mathrm{mT}$ on the layer of the circulator when the applied current is $300 \mathrm{~mA}$. Figure $4.22 \mathrm{~b}$ shows the magnetic field strength on the layer of the circulator's center conductor. This plot indicates that the field follows z-axis for a radius $\leq 0.6 \mathrm{~mm}$, and beyond that the field turns to the plane $\mathrm{x}-\mathrm{y}$.

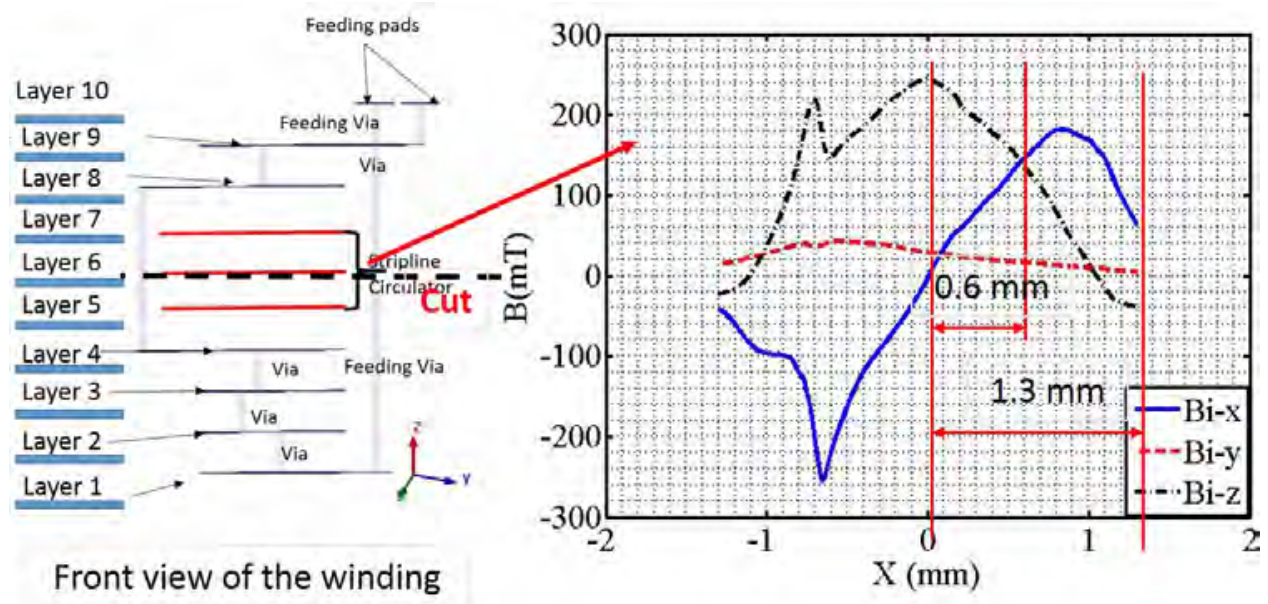

Figure 4.22b: Magneto-static simulation of winding in ferrite LTCC with a B-H curve: Bi along $\mathrm{X}$ axis on layer 6 (circulator inner conductor)

The reason for the lower than expected z-polarized magnetostatic field in the core of the circulator has to do with the flux return paths inside the ferrite medium. The ferrite-air boundary represents a high reluctance path, whereas the lateral (xy) paths inside the ferrite present a much lower reluctance to the flux. As such, the magnetostatic field tends to create a closed dipole field around the upper and lower coils separately instead of producing a mutual z-directed flux path between the coils, where the circulator is located. Furthermore, the xy flux paths are quite thin and tend to saturate first, which further limits the magnetostatic flux in the core.

Figure 4.22c shows the strength of the internal magnetic field along the $\mathrm{x}$-axis on layer 9. On this layer, most of the magnetic fields are in the xy-plane as we expect, and the strength is higher than $350 \mathrm{mT}$. In addition, the fields are strong near the 
winding conductors and they are less uniform than that in the middle of the winding. This illustrates the challenges when windings are designed in a homogeneous ferrite medium. Although more outer turns could be added to the coils, they would not add significantly more bias field to the core because the z-component is inversely proportional to the square of the distance. Higher flux densities could be obtained by adding more LTCC layers, but this adversely impacts the fabrication cost. Higher flux densities could also be obtained by using more turns per unit area, but this would require thinner lines that would pose a risk of melting when biased by the current. Using thinner layers $(58 \mu \mathrm{m})$ is another solution to get a higher flux densities [28], but the special fabrication process should be used. Hence the five turns coil represents a trade-off between the strength of the field created and the size of the coils.
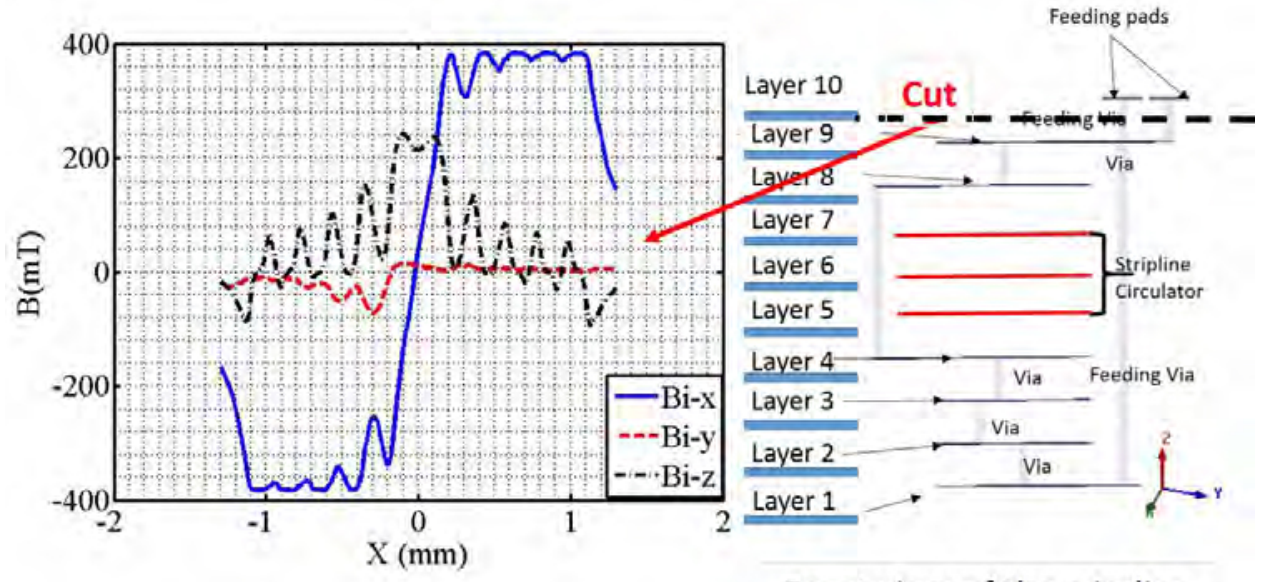

Front view of the winding

Figure 4.22c: Magneto-static simulation of winding in ferrite LTCC with a B-H curve: Bi along $\mathrm{X}$ axis on layer 9

The magnetization values obtained from the magnetostatic simulations could be used to calculate the elements of the permeability tensor. 


\subsubsection{Y-junction edge-guided circulator design}

The structure of the proposed circulator is shown in Figure 4.23. A stripline design is chosen specifically because the two broad metal ground planes allow the bias windings to be placed immediately above and below the circulator core without coupling to the microwave fields $[5,37]$. Hence the ground planes provide rf shielding while simultaneously allowing the magnetostatic bias field into the core. The proposed circulator uses the edge-guided mode of operation because of its known broadband performance $[59,60,122]$. The design of the circulator uses 10 layers of ESL 40012 ferrite LTCC, in which each layer has a fired thickness of $110 \mu \mathrm{m}$. The magnetostatic and microwave (X-band) properties of this ferrite have already presented: a saturation magnetization $\mu_{0} M_{s}=400 \mathrm{mT}$, a relative permittivity of $\varepsilon_{r}=14.6$, a loss tangent $4.24 \times 10^{-3}$. The damping factor $\alpha$ is found to be around 0.35 by measuring a CPW transmission line on ESL 40012 [94]. Hence the line width $\Delta H$ equals $250 \mathrm{Oe}$ according to Equation (4.17) [9]. This material is lossy below $10 \mathrm{GHz}$ due to the low-field losses, especially if it is totally demagnetized. The magnetization frequency is defined as $f_{m}=\gamma \mu_{0} M_{s}(11.2 \mathrm{GHz})$, so an operating frequency of $14 \mathrm{GHz}$ is chosen to avoid the low-field losses.

$$
\Delta H=\frac{2 \alpha \omega}{\mu_{0} \gamma}
$$

According to the characterization of this ferrite LTCC [15], integrated windings could generate a field which is less than the $M_{s}$ of the ferrite, leaving the material only partially magnetized. The theories of partially magnetized ferrites are given in $[85,86$, 89,91]. As we presented in the previous chapter, the model of Rado performs a spatial average of the domains in the ferrite [85]. For frequencies above the resonance, a good approximation for the extra-diagonal term $\kappa$ is obtained but not for the diagonal 


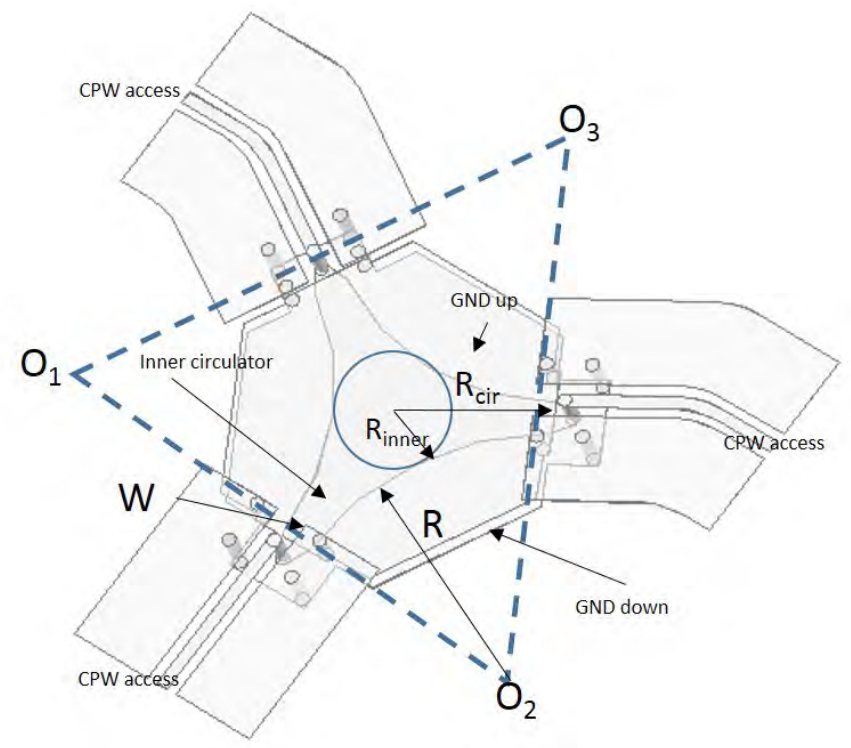

Figure 4.23: Edge-guided hexagonal circulator 3-D design for LTCC implementation

ones $\mu$ and $\mu_{z}$. An improved tensor model is proposed by Igarashi and Naïto [86], however, preliminary measurement should be performed to determine some variables, especially when the applied field is relatively high. A generalized permeability tensor (GPT) model which works for both saturated and partially magnetized ferrite is described [89]. The GPT model takes into account the demagnetizing field, the anisotropic field, the interactions between the adjacent domains, etc leading to a more rigorous tensor model. Due to a complex calculation, this model is difficult to be implemented into EM simulators. Therefore, we employ the Green and Sandy's tensor model in this design. The model is obtained based on the measurement of the experimental characterization cells. It is valid when the operating frequency is higher than the resonance which meets our requirements. Moreover, this model is easier to be implemented than the model of GPT. Finally, a tunable antenna on a partially magnetized ferrite LTCC substrate [28] used this permeability tensor model in [91] and obtained good theory-measurement agreement. In our design, the same ferrite LTCC substrate is used. The Green and Sandy's equations which explain the 
behavior of a partially magnetized ferrite material when using Z-bias (magnetic bias perpendicular to the circulators plane) are given by Equations (4.18) to (4.23), where $\mu_{0}^{\prime}$ is the initial relative permeability of the substrate in the demagnetized state, the relative $\mu$ and $\kappa$ values replace the standard elements of the Polder permeability tensor, $\mathrm{M}$ and $M_{s}$ are the partial and saturation magnetization, $\mathrm{f}$ is the circulator's working frequency and $\mathrm{n}$ is the coefficient expressing the deviation of $\kappa$ from Rado's model, which is assumed for this work to be 0.5 since the same ferrite LTCC material ESL 40012 is used [28].

$$
\begin{gathered}
\text { Permeability }=\mu_{0}\left[\begin{array}{ccc}
\mu & -j \kappa & 0 \\
j \kappa & \mu & 0 \\
0 & 0 & \mu_{z}
\end{array}\right] \\
\mu_{0}^{\prime}=\frac{2}{3}\left[\begin{array}{cc}
1-\left(\frac{\gamma 4 \pi M_{s}}{\omega}\right)^{2} \\
\mu=\mu_{0}^{\prime}+\left(1-\frac{1}{3}\right. \\
\left.\mu_{0}^{\prime}\right)\left(\frac{M}{M_{s}}\right)^{1.5} \\
\mu_{e f f}=\frac{\mu^{2}-\kappa^{2}}{\mu} \\
\mu_{z}=\mu_{0}^{\prime}\left(1-\left(\frac{M^{2}}{M_{s}}\right)^{2.5}\right.
\end{array}\right)
\end{gathered}
$$

The circulator's working frequency could be calculated by Equation (4.24) for a 
given value of the circulator's radius $R_{\text {cir }}$ (Figure 4.23), where the $\mu_{0}$ is the permeability of free space, $\varepsilon_{f}$ is the permittivity of the ferrite, $\epsilon_{0}$ is the permittivity of the space and $\mu_{e f f}$ is the effective permeability of the ferrite [37].

$$
f=\frac{1.84}{2 \pi R_{\text {cir }} \sqrt{\varepsilon_{0} \mu_{0} \varepsilon_{f} \mu_{e f f}}}
$$

The value of $R_{c i r}$ assumed for this work is $1.79 \mathrm{~mm}$ which is chosen for a circulator working at $14 \mathrm{GHz}$ as shown in Figure 4.24. It is obtained by Matlab using Green and Sandy's partially magnetized ferrite tensor when magnetization $\mu \mathrm{M}=200 \mathrm{mT}$. This magnetization value is the maximum value created by the winding as discussed in the winding's design section. The reason why we use the approximate dimensions of a Bosma theory is that it gives us the smallest radius of the circulator (principal mode of the circulation). As is known, a small bias area requires reduced bias energy.

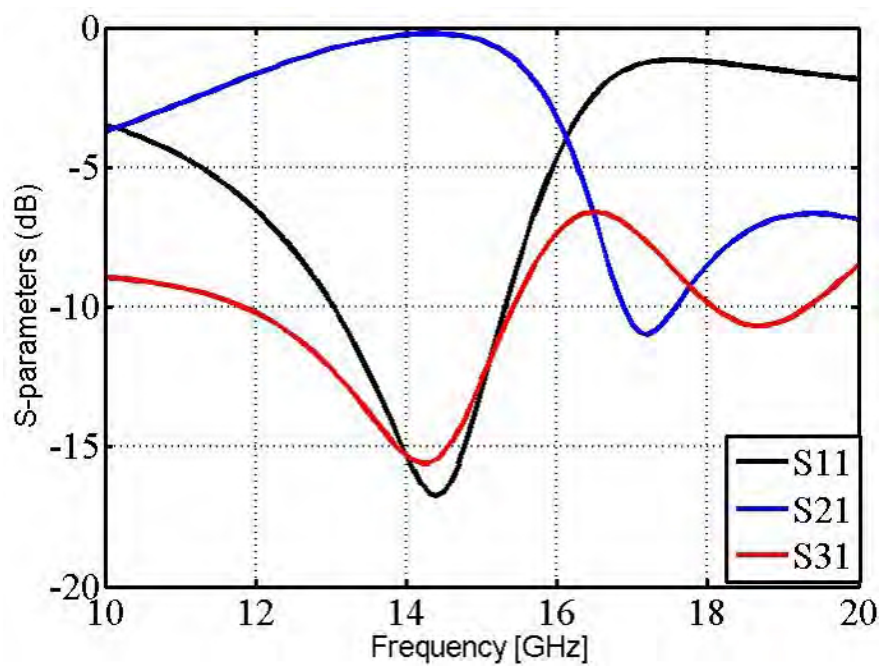

Figure 4.24: The simulated S-parameters in Matlab by employing Green and Sandy's partially magnetized tensor model when $\mu \mathrm{M}=200 \mathrm{mT}$ and $\mathrm{R}=1.79 \mathrm{~mm}$

When we vary the internal magnetization $\mu \mathrm{M}$, the circulation under different $\mu \mathrm{M}$ could be shown in Figure 4.25. When $\mu \mathrm{M}=0$, there is a superposition of $\mathrm{S} 21$ and $\mathrm{S} 12$ curve meaning that the circulator works as a power divider. When we increase the 
internal $\mu \mathrm{M}$ gradually, we create the circulation (separate S12 and S21) along with a frequency variation.

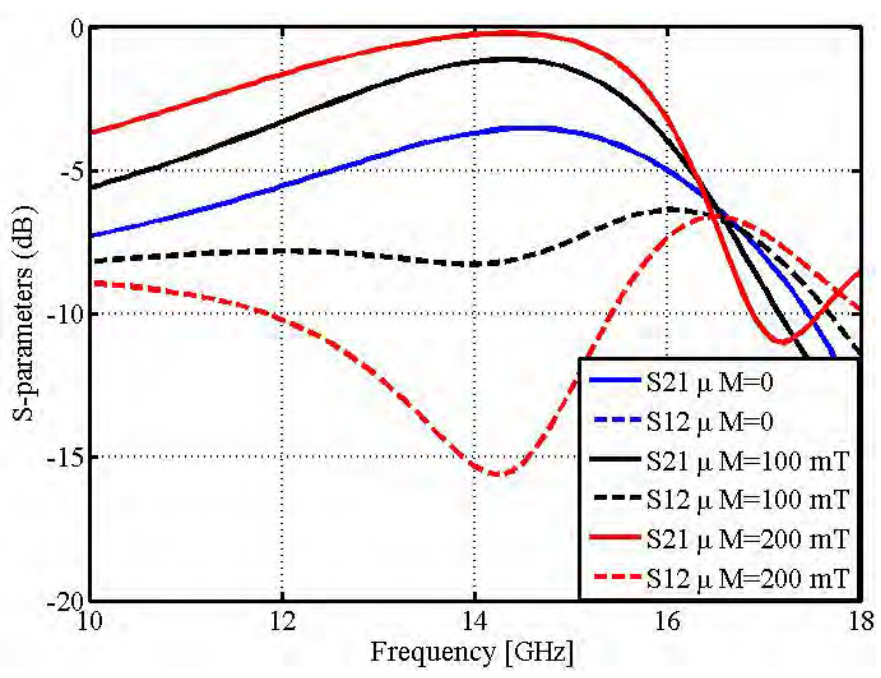

Figure 4.25: The simulated S-parameters in Matlab with Green and Sandy's partially magnetized tensor model under different internal magnetization $\mu \mathrm{M}$

Figure 4.26 demonstrates that in a partially magnetized ferrite, the circulator's working frequency is in inverse proportion to $\mu \mathrm{M}$ when $\mathrm{n}=0.5$. The frequency decreases from $14.6 \mathrm{GHz}$ to $14.2 \mathrm{GHz}$ when $\mu \mathrm{M}$ changes from 0 to $0.2 \mathrm{~T}$ leading to a variation of $0.4 \mathrm{GHz}$.

\section{EM simulation by Ansys HFSS 15 (Ansys Electromagnetics Suite)}

Figure 4.21a shows the stack-up of the 10 LTCC layers. As we said before, space priority is given to the dc windings, so the circulator is designed using a minimum number of layers: the stripline ground planes are on layers 5 and 7 , whereas the circulator's inner conductor is on layer 6 . Referring to Figure 4.23, key parameters of an edge-guided circulator are the radius of curvature $\mathrm{R}$ and the width $\mathrm{W}$ of the center conductor at the circulator's edge. The next difficulty consists in feeding the embedded circulator because it can only be probed on the top layer (layer 10). To 


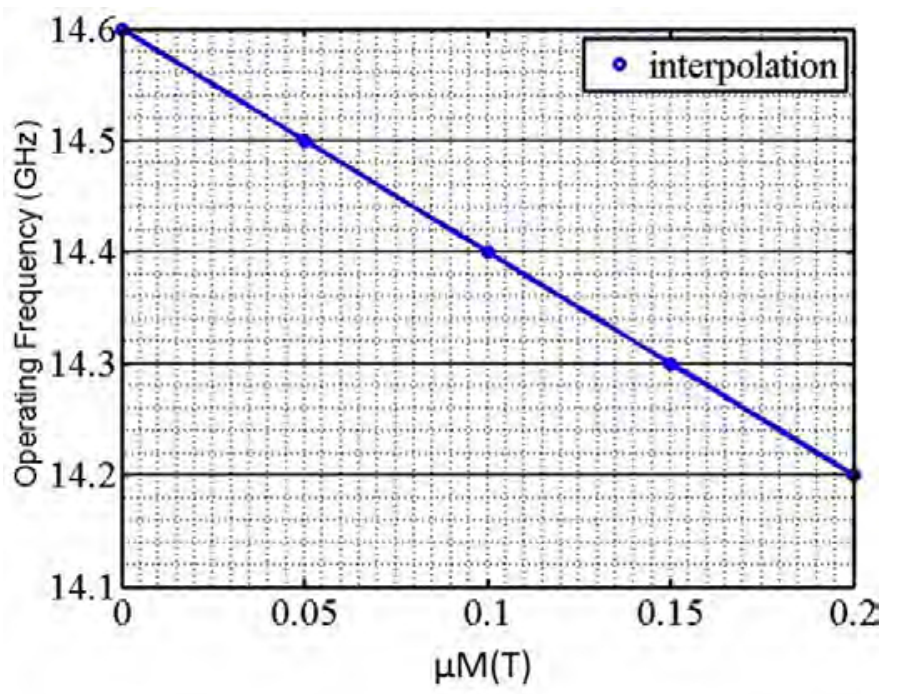

Figure 4.26: The circulator's working frequency in terms of the internal magnetization $\mu \mathrm{M}$

solve this problem, three CPW transmission feed lines are designed on the top layer. The transition from the CPW feed lines to the embedded stripline circulator is shown in Figure 4.27, where five stacked vias are used to connect the signal lines and the ground planes. Both of the stripline ground planes are bonded by the vias to avoid exciting parasitic parallel plate modes.

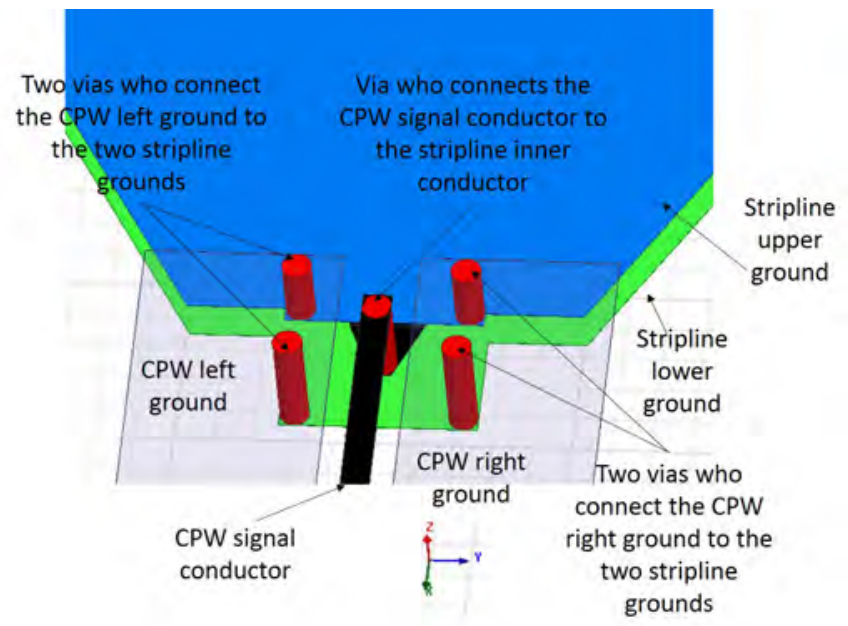

Figure 4.27: Transition from CPW transmission line to stripline 
The simulator Ansys HFSS has predefined Polder equations for the ferrite's permeability tensor, and this is the only model allowed in HFSS. However, Polder model only works for saturated ferrites [9]. At that moment, we had only this simulator, so we performed the simulation anyway. In order to complete an approximate simulation for partially magnetized ferrites in HFSS, the magnetization M could be used instead of saturation magnetization $M_{s}$ along with the corresponding internal $H_{i}$ bias which can be found in [15]. For example, the circulator simulated above in Matlab has a partial magnetization $\mu M=0.2 \mathrm{~T}$, so the $\mu_{0} M_{s}=400 \mathrm{mT}$ is replaced by $\mu M=0.2$ $\mathrm{T}$ and the $H_{i}$ is set to be $500 \mathrm{~A} / \mathrm{m}$. This method allows to give an approximate simulation.

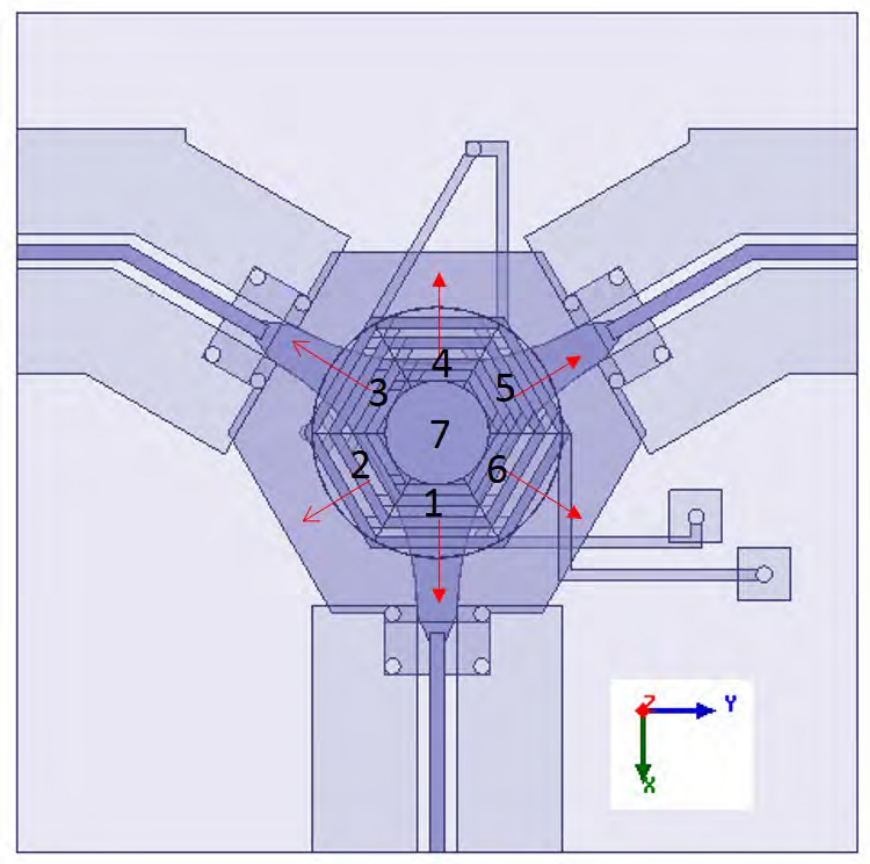

Figure 4.28a: Magnetic bias model in HFSS

In order to take the non-uniform magnetic field into account, a set of biased ferrites is manually created. Figure $4.22 \mathrm{~b}$ shows that the $\vec{B}_{i}$ vector has a dominant z-direction when x ranges from 0 to $0.6 \mathrm{~mm}$. Then $\vec{B}_{i}$ vector turns towards to the x-direction 
(in xy plane) gradually for $\mathrm{x}$ from $0.6 \mathrm{~mm}$ to $1.3 \mathrm{~mm}$. According to these features, the magnetic bias is designed to have 7 parts in HFSS as shown in Figure 4.28a. Part 7 is the center bias following the z-axis with an average value of $199 \mathrm{mT}$. Parts 1- 6 have the fields who have radial directions from the center (red arrows) with an average value of $132 \mathrm{mT}$. As known, HFSS allows non-uniform magnetic bias by using a co-simulation with Maxwell 3D. However, HFSS employs only Polder's equations to calculate the permeability tensor, therefore, we have to define the $M_{s}$ of the ferrites in HFSS. Obviously, a $\mu_{0} M_{s}=0.4 \mathrm{~T}$ is not accurate to model a partially magnetized ferrite. That's the reason why we use the model of 7 parts of ferrites whose $M_{s}$ are replaced by M. The circulator is then optimized using Ansys HFSS which led to the final dimensions of the circulator: $\mathrm{R}=2.8 \mathrm{~mm}$ and $\mathrm{W}=0.38 \mathrm{~mm}$, so $R_{\text {cir }}$ equals $1.73 \mathrm{~mm}$ which is in agreement with the Matlab analysis $\left(R_{c i r}=1.79 \mathrm{~mm}\right)$.

The simulated transmission coefficients by HFSS and Matlab are shown in Figure 4.28b. The simulation in Matlab uses Green and Sandy's model, and HFSS uses Polder's equations with 7 parts of ferrites whose $M_{s}$ are replaced by M. Although the comparison seems to be unnecessary because they use totally two different ferrite models, however, this tells that the approximate model in HFSS still works to some extent. They both give a circulation at $14 \mathrm{GHz}$ while Matlab has a better insertion loss.

According to the contact with Ansys, HFSS will probably allow user-defined tensor model such as ferrite permeability tensor in the version of 2016 .

\subsubsection{Measurements}

The fabrication is performed by VTT Technical Research Centre of Finland with the help of Dr Atif Shamim at King Abdullah University of Science and Technology 


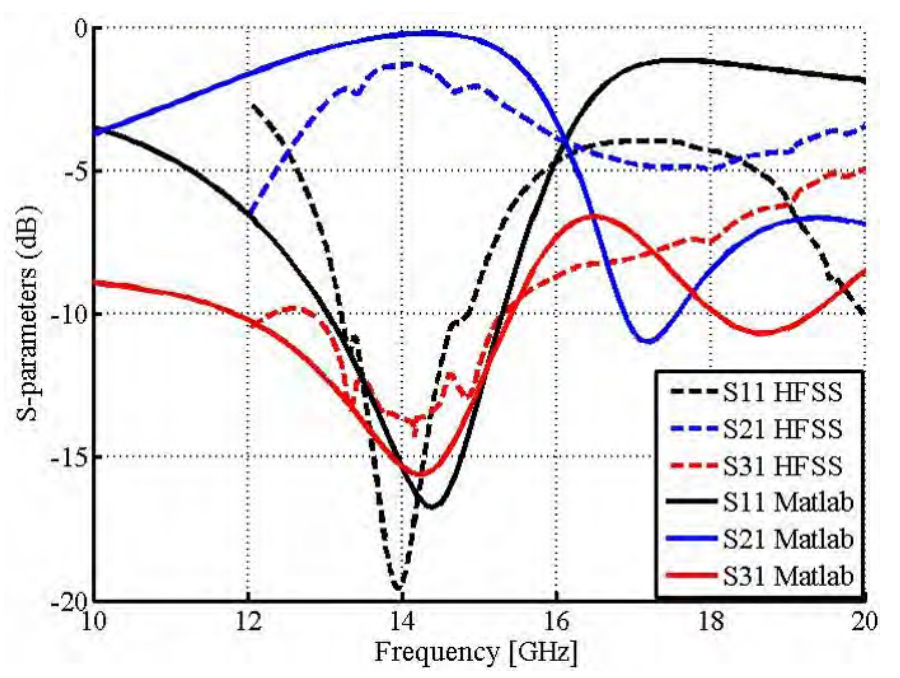

Figure 4.28b: Simulated S-parameters in HFSS and Matlab

in Saudi Arabia. A photograph of the three fabricated ferrite LTCC edge-guided circulators and one CPW thru line of $2.7 \mathrm{~mm}$ are shown in Figure 4.29. Note how the three ports of the circulator have been arranged in a $\mathrm{T}$ shape to accommodate the probing station's perpendicular probe arms. A bend in two of the CPW lines is therefore required and care is taken to ensure that these bends would not cause reflections near $14 \mathrm{GHz}$ (see Figure 4.30). Two GSG rf probes $(500 \mu \mathrm{m})$ are used to connect the port 1 and 2. An external dc source is connected to the dc pads by two dc-needles. An ampere meter is used to show the current strength in the winding. The network analyzer is the Agilent (Keysight) N5244A, 4-port (dual source) to 43.5 GHz. The measurement of a CPW thru is shown in Figure 4.31, which shows that the performance of the unbiased LTCC material is good between $10 \mathrm{GHz}$ and $17 \mathrm{GHz}$. As expected in [121], the attenuation is indeed strong below $10 \mathrm{GHz}$ due to the low-field losses. 


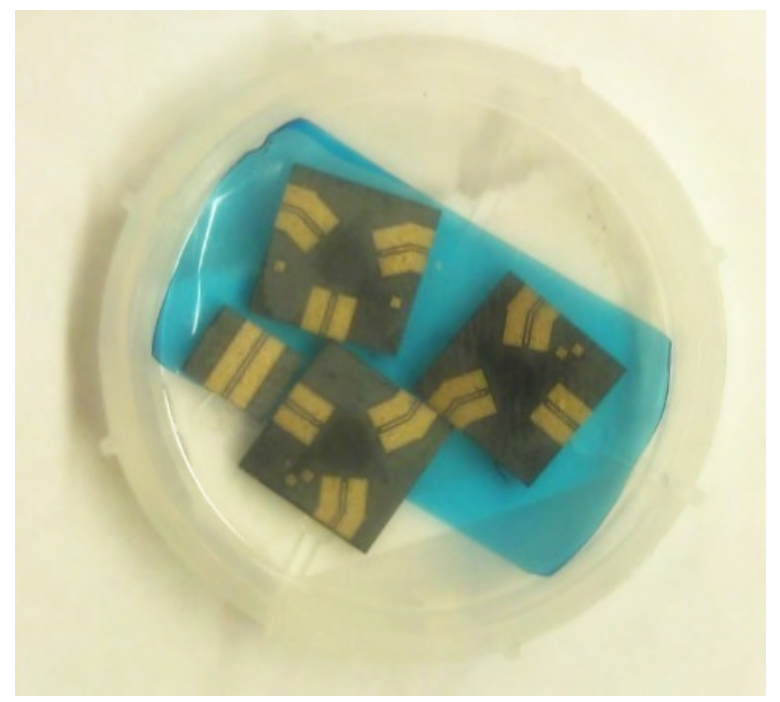

Figure 4.29: Prototypes of the ferrite LTCC circulator

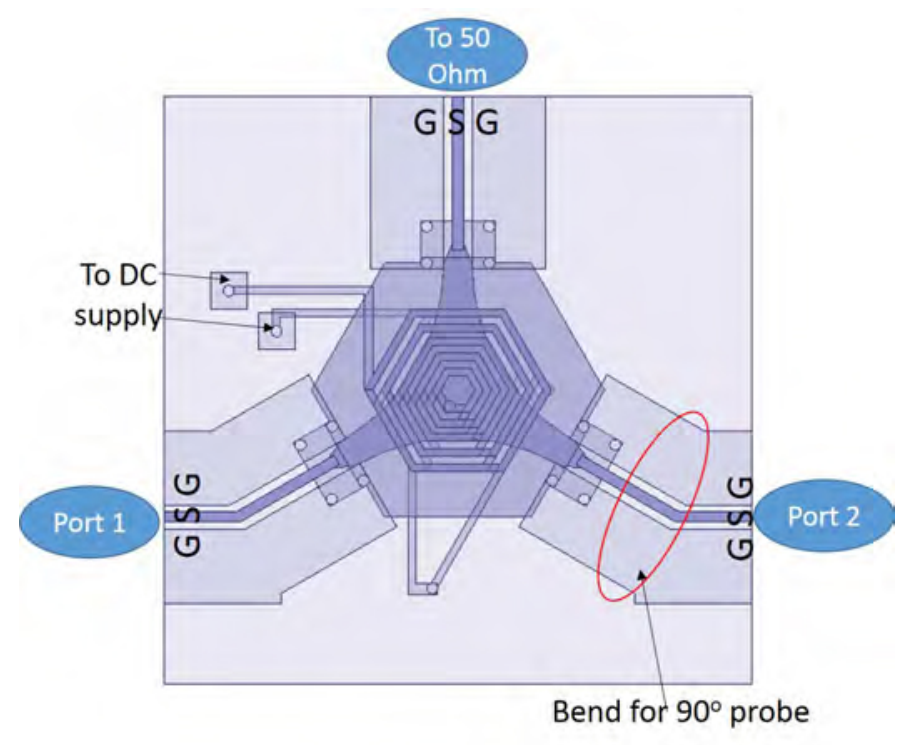

Figure 4.30: S-parameters measurement configuration

\section{Measurement with bias current only}

The measurement of the circulators is performed with a two-port network analyzer, with the third port (North) being matched $(50 \Omega)$. When the windings are not energized, a matching point is found at $14.8 \mathrm{GHz}$ as shown in Figure 4.32. The transmission and the isolation are identical with a value of $-5 \mathrm{~dB}$. This indicates that the 


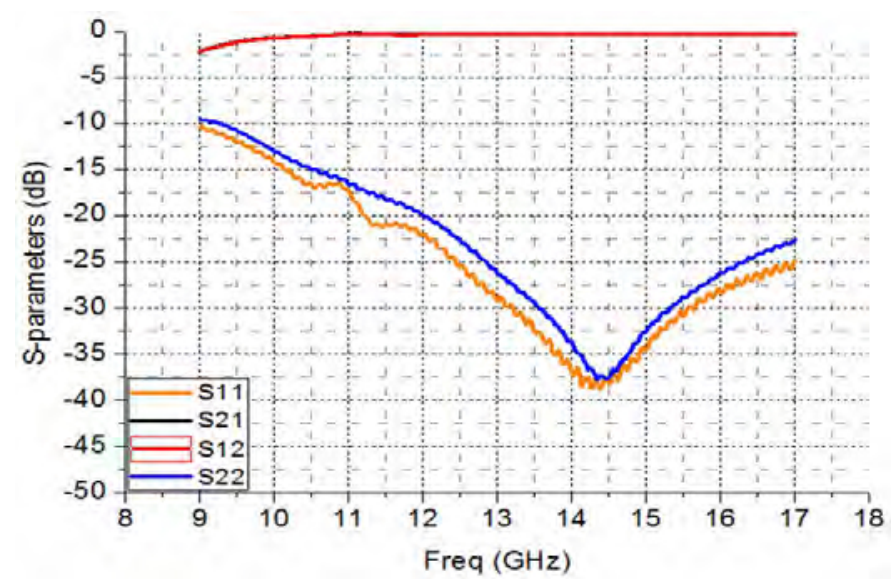

Figure 4.31: CPW transmission line measurement: S-parameters

circulator operates as a power divider (with losses) when it is not biased. There is a loss of $2 \mathrm{~dB}$ because the material has a loss of $1.5 \mathrm{~dB} / \mathrm{cm}$ at $14 \mathrm{GHz}$ for a transmission line [15]. The input-to-output length of our circulator is about $8.6 \mathrm{~mm}$, so the loss is approximately $1.5 * 0.86=1.3 \mathrm{~dB}$ which is in agreement with the measurement.

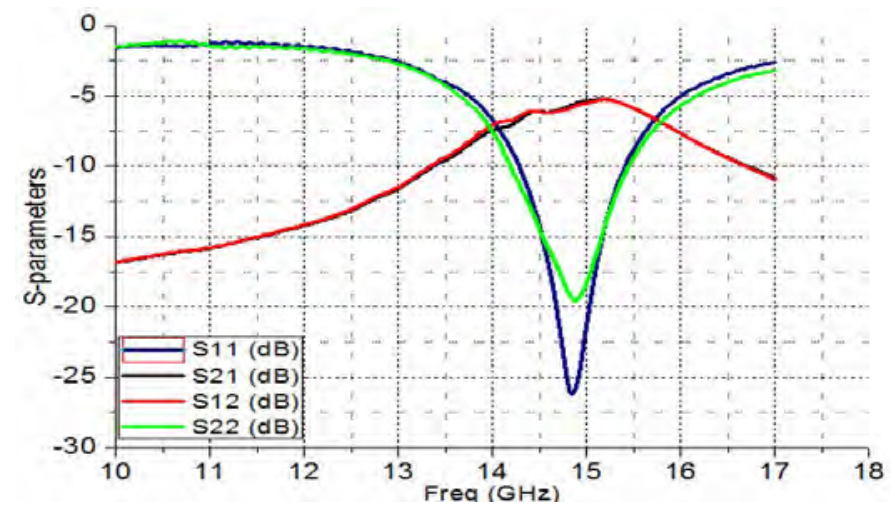

Figure 4.32: Measured S-parameters: Circulator without current

When a current of $100 \mathrm{~mA}$ is injected into the winding, circulation becomes apparent (see Figure 4.33). The transmission is pushed up to $-4 \mathrm{~dB}$ and the isolation goes down to $-7 \mathrm{~dB}$ at $14.67 \mathrm{GHz}$, whereas the reflections remain below $-20 \mathrm{~dB}$. When the current is increased to $300 \mathrm{~mA}$, the performance of the circulator is further improved (see Figure 4.33). The transmission is about $-3 \mathrm{~dB}$ and the isolation about $-8 \mathrm{~dB}$ 
at $14.2 \mathrm{GHz}$. A stronger current may be applied, but this could melt the winding. Moreover, the operating frequency is shifted as well from $14.8 \mathrm{GHz}$ to $14.2 \mathrm{GHz}$ in the measurement. We believed at that moment that the frequency variation was caused by the change of the internal magnetization $\mathrm{M}$ as expected in Matlab, however, this is finally proven that it is mainly caused by the temperature rise in the substrate and we will explain it later.

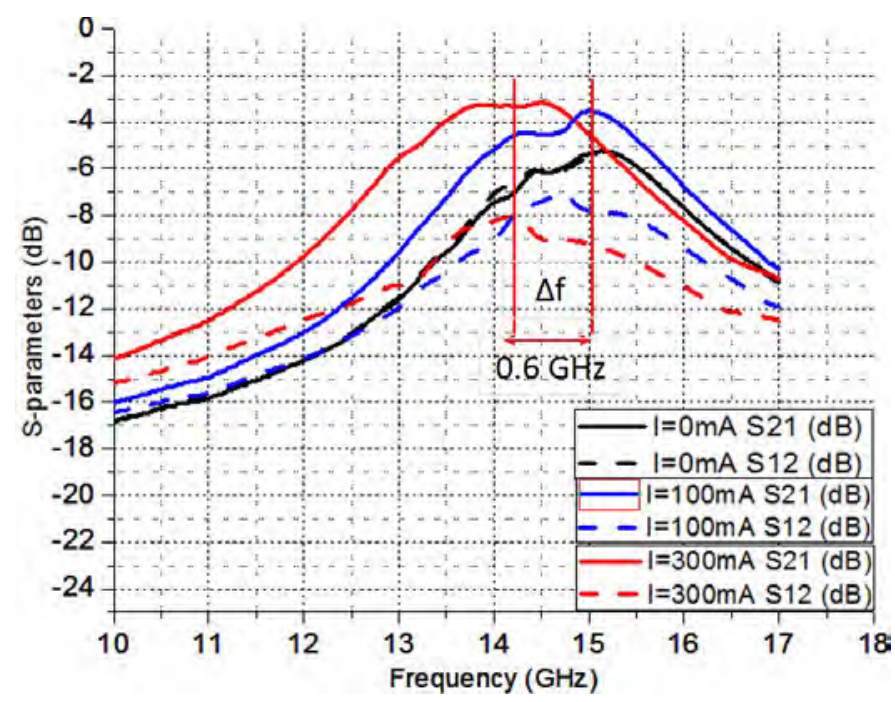

Figure 4.33: Measured S-parameters with current: S21 and S12 in terms of current

Figure 4.34a shows the comparison of simulated S21 and S12 between HFSS and the measurement, when a $300 \mathrm{~mA}$ current is injected into the winding. HFSS gives better insertion loss and isolation than the measurement. This is probably due to the additional losses of a partially magnetized ferrite and the inaccurate ferrite model in HFSS.

However, the simulation has a good agreement with the measurement in terms of reflections S11 and S22, as shown in Figure 4.34b. The circulator's operating frequency is also well predicted by the simulation. 


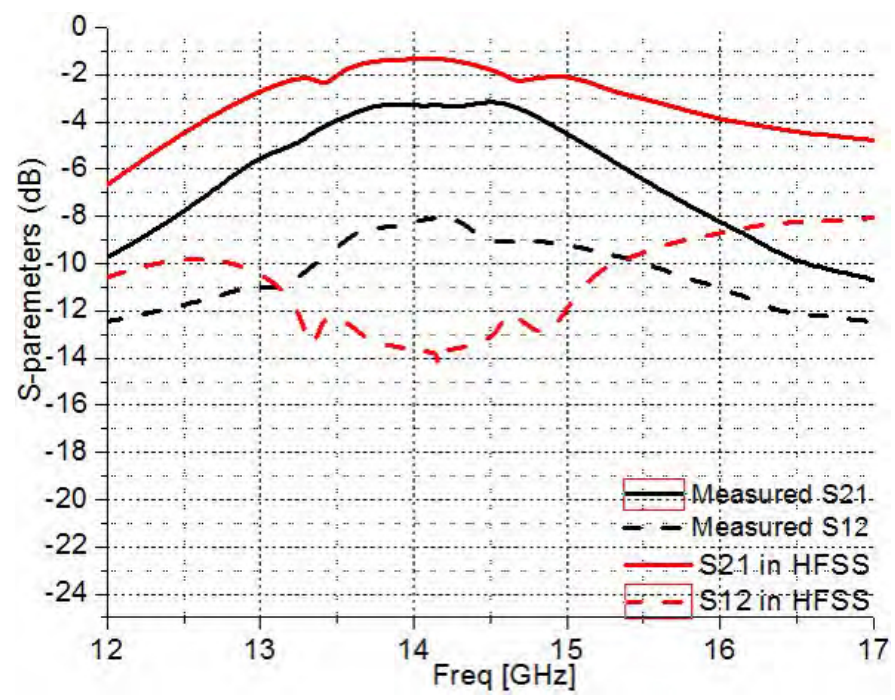

Figure 4.34a: Comparison of measurement and HFSS: S21 and S12

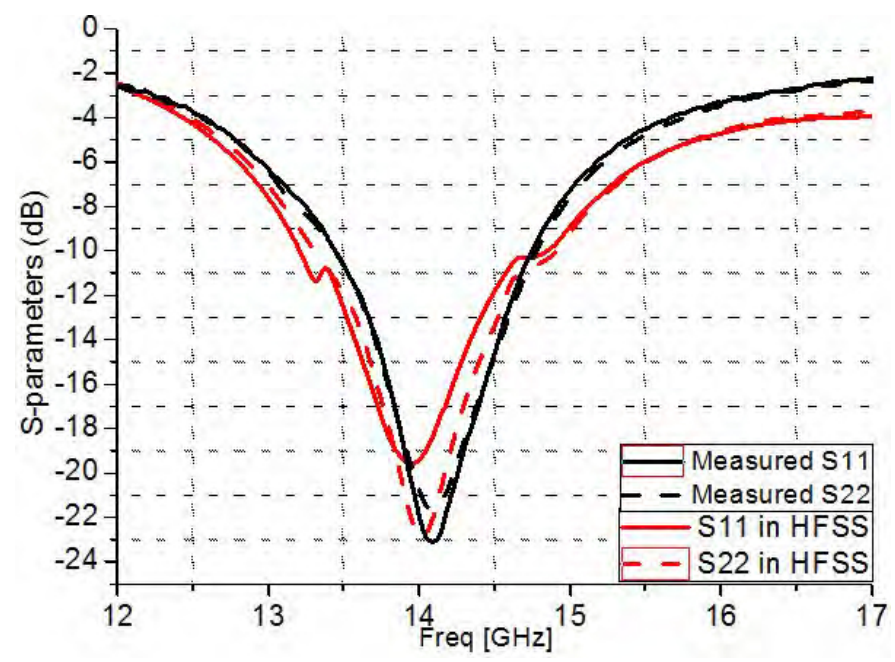

Figure 4.34b: Comparison of measurement and HFSS: S11 and S22

In addition, Figure 4.34c shows the S-parameters when the current is switched. As expected, the circulators' circulation is switched to the opposite direction.

\section{Measurement with magnets}

The measurement with the current confirms that the circulator is not well saturated using only the windings, so measurements with external magnets are performed as 


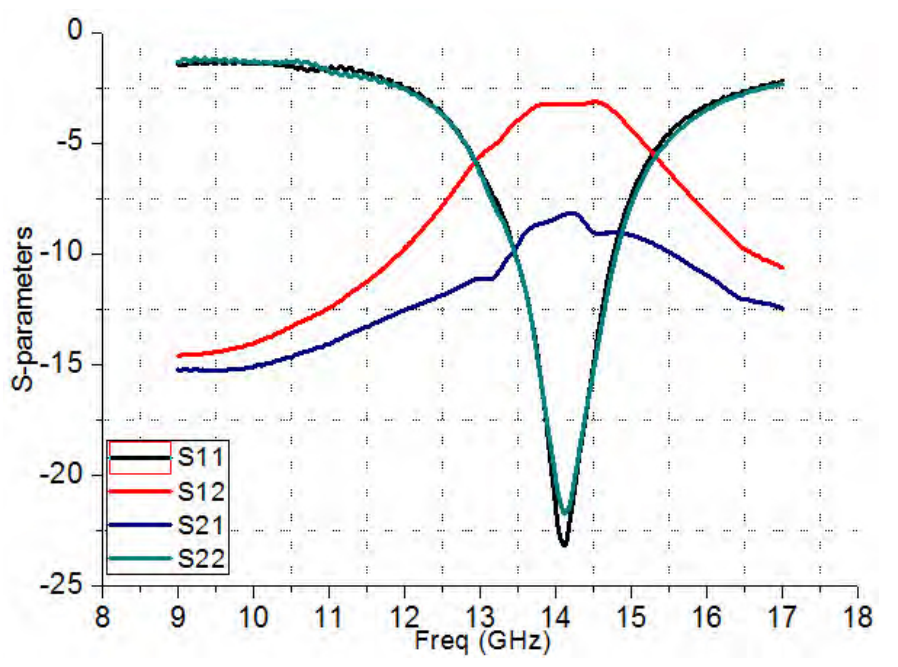

Figure 4.34c: Circulator with an opposite direction of $300 \mathrm{~mA}$ dc current: Sparameters

well. An external magnet with a surface field of 5200 Gauss plus a second one with 5903 Gauss surface field are applied on the top layer of the substrate and the bias diameter is 1/16 inch $(1.5875 \mathrm{~mm})$, as shown in Figure 4.35 .

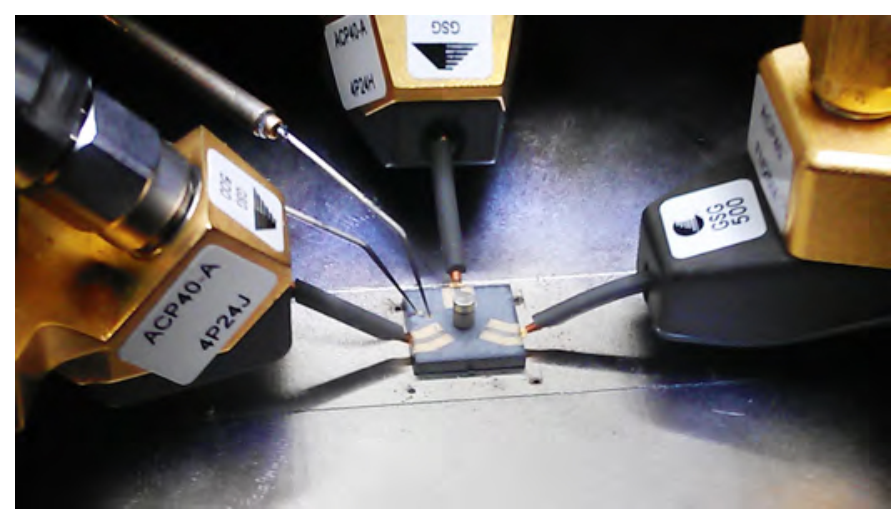

Figure 4.35: Measurement probe station photo

Figures $4.36 \mathrm{a}$ and $4.36 \mathrm{~b}$ shows that the internal magnetic field $\vec{B}_{i}$ is higher than $400 \mathrm{mT}$ in the core of the circulator for a radius from 0 to $0.5 \mathrm{~mm}$, therefore, the magnets could saturate the ferrite. 


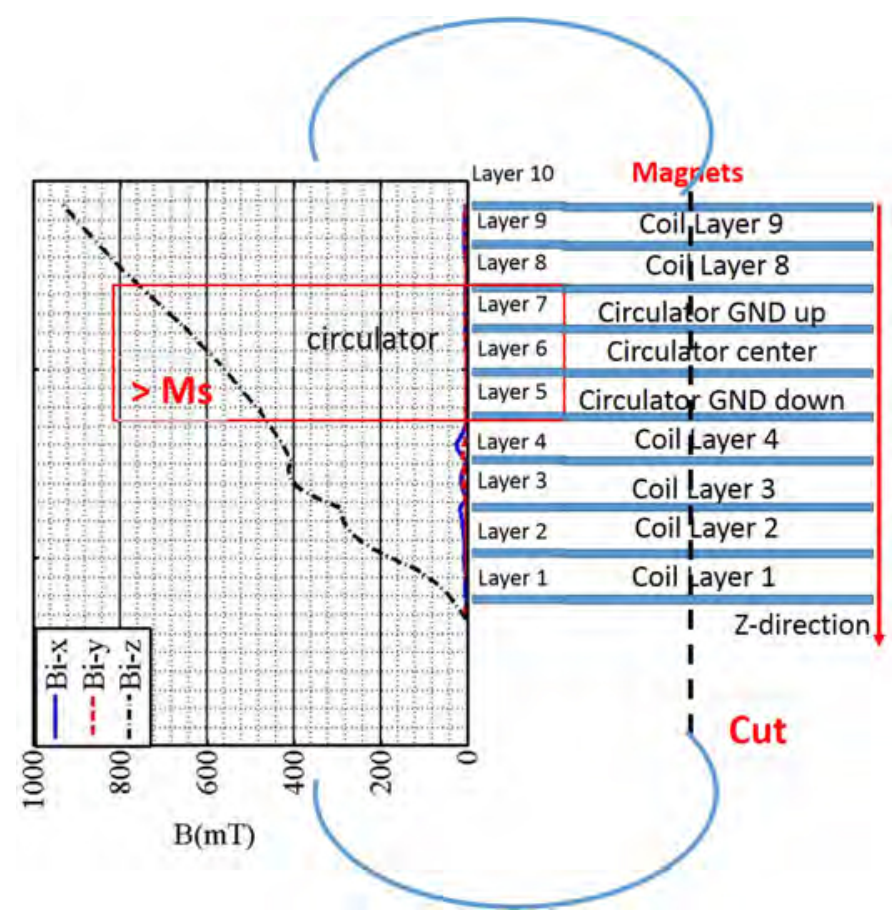

Figure 4.36a: Magneto-static simulation of the magnets in ferrite LTCC with a B-H curve: $\mathrm{Bi}$ along $\mathrm{Z}$ axis

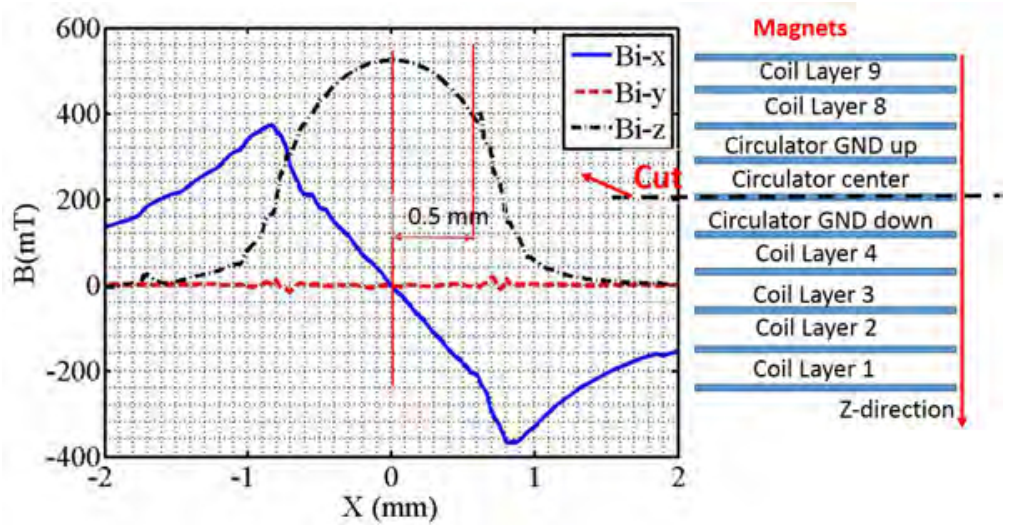

Figure 4.36b: Magneto-static simulation of the magnets in ferrite LTCC with a B-H curve: Bi along $\mathrm{X}$ axis

Then we performed the measurements with the external magnets. The magnets greatly improve the performance of the circulator. The measured transmission and isolation is approximately $-1.8 \mathrm{~dB}$ and $-18 \mathrm{~dB}$, respectively, when current $\mathrm{I}=0 \mathrm{~mA}$ is injected, as shown in Figure 4.37a, while the return loss is better than $20 \mathrm{~dB}$ 
(Figure 4.37b).

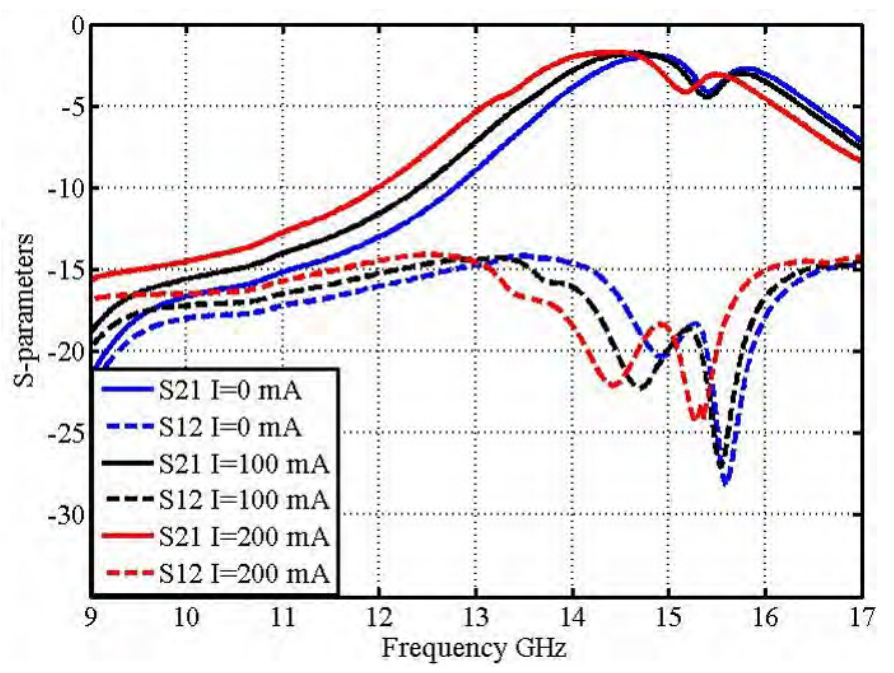

Figure 4.37a: S-parameters with magnets: S21 and S12 in terms of current

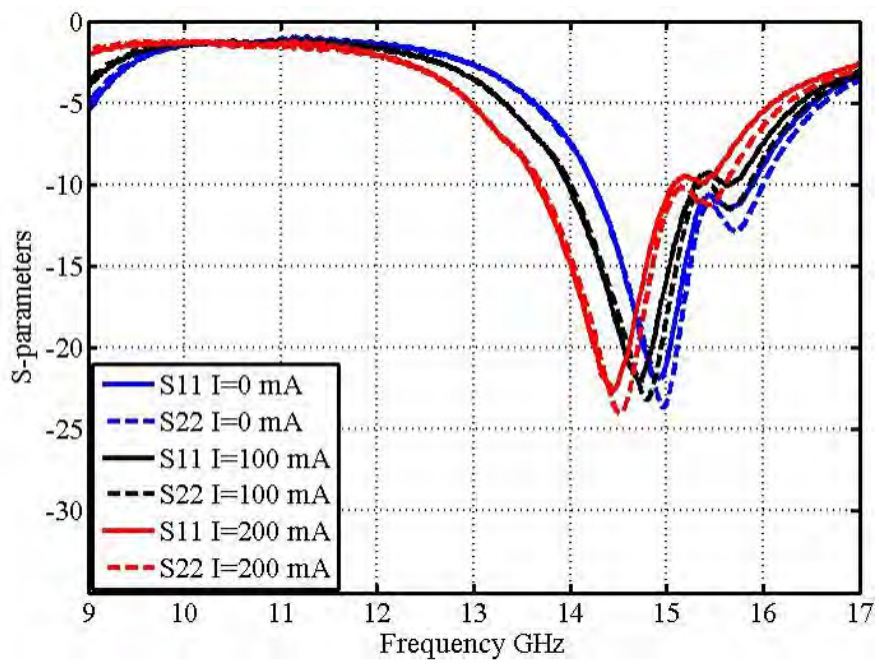

Figure 4.37b: S-parameters with magnets: S11 and S22 in terms of current

With the magnets, the internal magnetization can be adjusted by changing the current in the windings. When a current of $100 \mathrm{~mA}$ is applied to the windings (the $\mathrm{H}$ field created by the winding has the same direction of magnet's field), the circulator performance is shown in Figure 4.37a and Figure 4.37b. The isolation goes down to 
$-22 \mathrm{~dB}$ instead of $-18 \mathrm{~dB}$. When the current is increased to be $200 \mathrm{~mA}$, the result is further improved. The transmission is about $-1.6 \mathrm{~dB}$, isolation is $-23 \mathrm{~dB}$ and return loss is better than $-20 \mathrm{~dB}$. However, in this measurement, a frequency shift is also observed: from $14.8 \mathrm{GHz}$ to $14.2 \mathrm{GHz}$.

This is interesting: we said before that the frequency variation was caused by the change of the internal magnetization M. However, in this measurement, the field created by the magnets is so strong that the field created by the winding could be negligible. Why do we still observe a frequency variation of $0.6 \mathrm{GHz}$ ? The hypothesis is that the temperature also affects the frequency variation. To prove that, we performed another measurement.

This time, the field created by the winding is opposite to the field of the magnets. We find that the frequency goes up a little when $120 \mathrm{~mA}$ current is injected, then the frequency goes down again if we continue increasing the current, as shown in Figure 4.37c. This measurement indicates that the variation of the circulator's frequency is not only caused by the change of the internal magnetization. The explanation of the frequency variation is that there are two elements that change the circulator's operating frequency: the first is the change of magnetization M (hence $\left.\mu_{e f f}\right)$ by the winding, the second is the temperature rise in the substrate which affects $\mu$ and $M_{s}$ leading to the variation of $\mu_{e f f}$. The temperature increases the ferrites $\mu$ as explained in [91] leading to the increase of $\mu_{e f f}$. Moreover, the temperature decreases the magnetization saturation $M_{s}$ which increases the $\mu_{\text {eff }}$ as well when the ferrite is partially magnetized (Figure $4.37 \mathrm{~d}$ ). In conclusion, the frequency variation is achieved initially by the change of internal magnetization $\mathrm{M}$, then the temperature plays a more important role in changing the $\mu_{e f f}$, as this ferrite LTCC substrate ESL 40012 is sensitive to the temperature. 


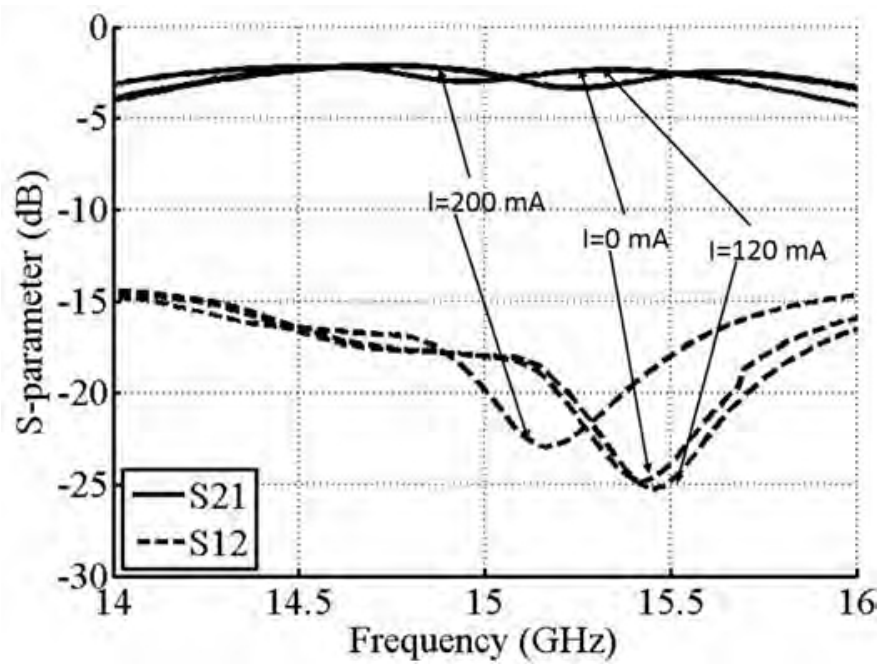

Figure 4.37c: Isolation and transmission curves when windings with the current is biased that opposes the magnetic fields of the magnet

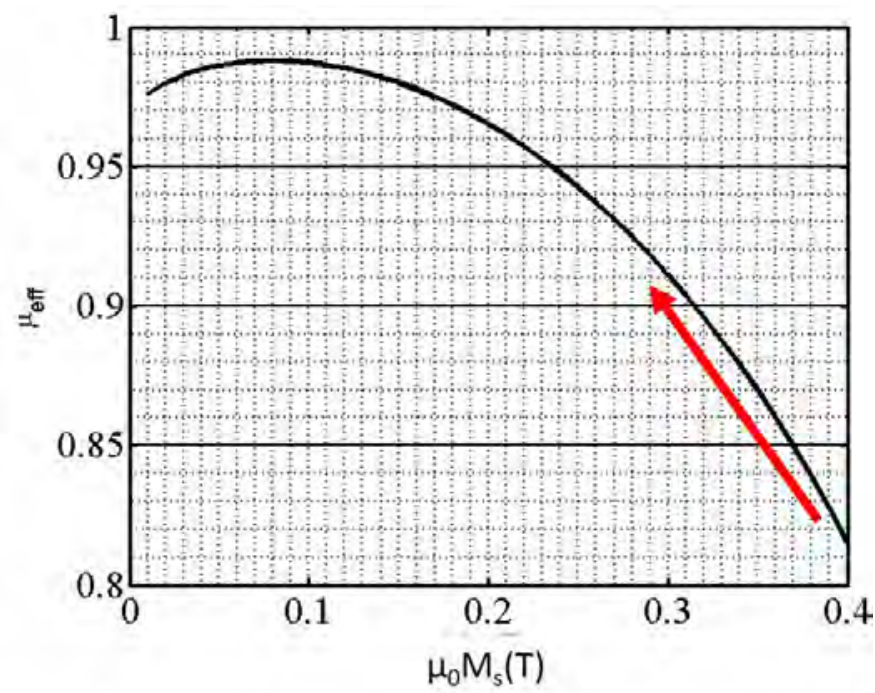

Figure 4.37d: $\mu_{\text {eff }}$ in terms of magnetization saturation $\mu_{0} M_{s}$ with $\mu M=0.2 T$ when the ferrite is partially magnetized using the model of Green and Sandy

\subsubsection{Retro Analysis}

\section{EM simulation by CST Studio}

As discussed before, the simulator Ansys HFSS uses the Polder equations [123] to simulate saturated ferrites. This year, we finally have the access to CST Studio which 
allows one to apply user-defined tensors manually [124], therefore we performed retro analysis with Green and Sandy's partially magnetized ferrite model in CST Studio, as shown in Figure 4.38a. The side-to-side length of the winding is $2.2 \mathrm{~mm}$, so the radius of the bias area is set to be $1.27 \mathrm{~mm}$. The remaining ferrite is assumed to be totally demagnetized with $M=0$ and $M_{s}=0$.

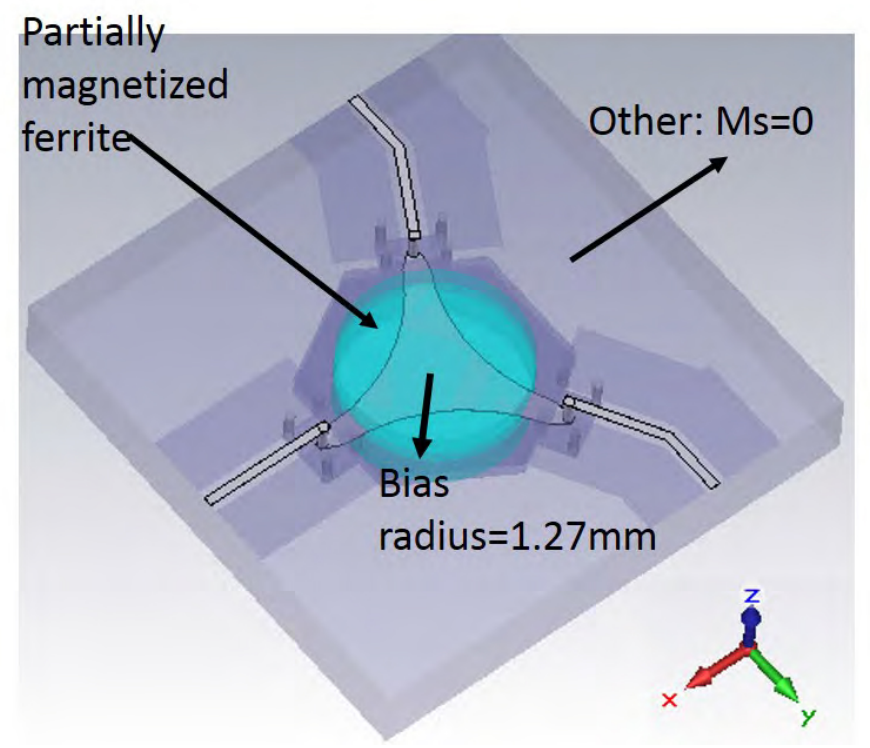

Figure 4.38a: Magnetic bias model in CST

Then the simulation is performed and we compare the simulated S-parameters with the measured ones when the internal magnetization $\mu \mathrm{M}$ is $200 \mathrm{mT}$, as shown in Figures 4.38b and 4.38c. CST gives a more close result for the transmission and isolation, however, the reflection is not accurate which is probably because we used a simplified and uniform bias field. However, the working frequency is also well predicted. At $14 \mathrm{GHz}$, the transmission and isolation is $-3 \mathrm{~dB}$ and $-9 \mathrm{~dB}$ respectively. More important, since Green and Sandy's partially magnetized ferrite model is used, we can perform the simulations with different internal magnetization $\mu \mathrm{M}$. A frequency variation of $0.2 \mathrm{GHz}$ is then observed when $\mu \mathrm{M}$ goes from 0 to $200 \mathrm{mT}$, as shown 


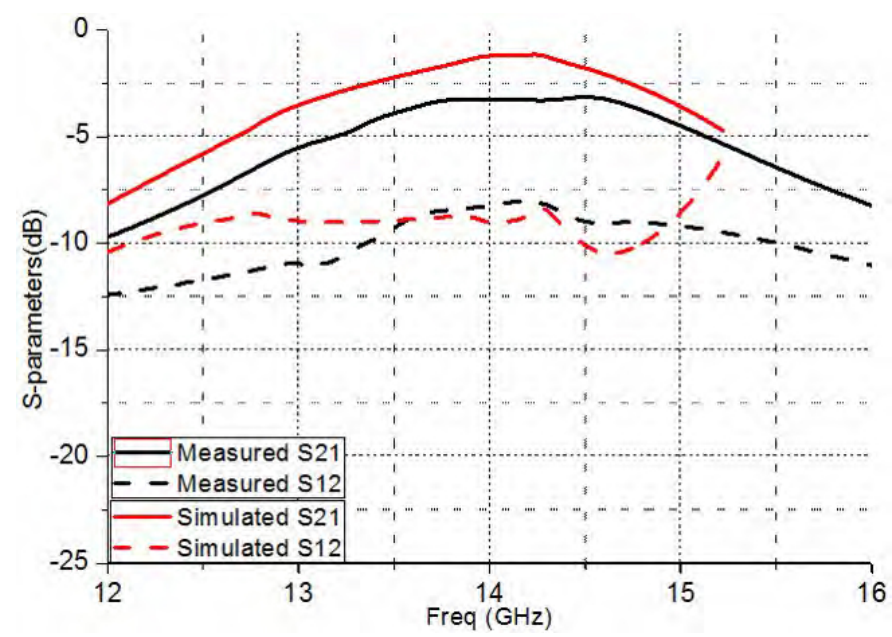

Figure 4.38b: Comparison of measured and simulated S21 and S12 by CST when internal magnetization $\mu \mathrm{M}$ is $200 \mathrm{mT}$

in Figure 4.38d. However, in the measurement, the variation is $0.6 \mathrm{GHz}$ and the difference is due to the temperature rise in the substrate.

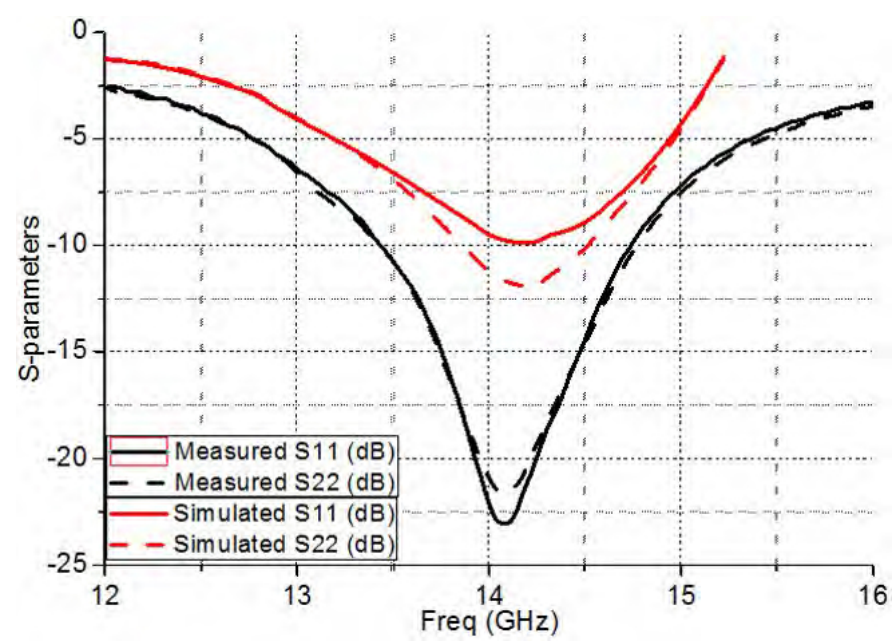

Figure 4.38c: Comparison of measured and simulated S11 and S22 by CST when internal magnetization $\mu \mathrm{M}$ is $200 \mathrm{mT}$

The final dimensions of the circulator: $\mathrm{R}=2.8 \mathrm{~mm}$ and $\mathrm{W}=0.38 \mathrm{~mm}$, so $R_{\text {cir }}$ equals $1.73 \mathrm{~mm}$ which is in agreement with the Matlab analysis $\left(R_{c i r}=1.79 \mathrm{~mm}\right)$. 


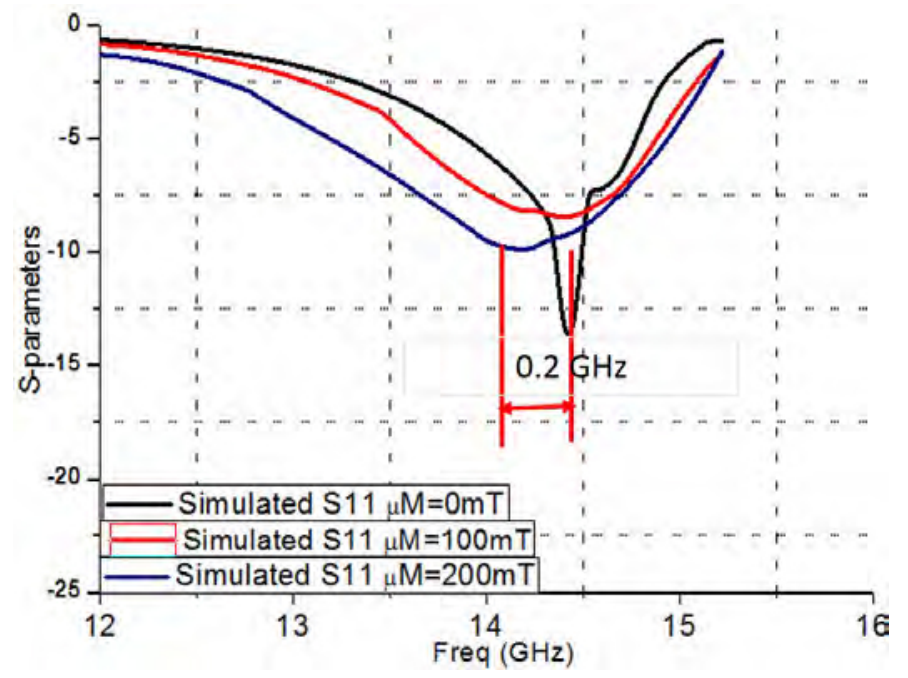

Figure 4.38d: Simulated circulator's frequency variation (S11 in dB) in terms of magnetization $\mu \mathrm{M}$

\subsubsection{Power Capability IP3}

Given that ferrite devices are perceived as being nonlinear, so it is necessary to measure the third-order intercept point of a circulator made of ESL 40012 ferrite LTCC.

A two tone test is applied to the circulator in an attempt to measure its thirdorder intercept point. The 10-layer ESL 40012 ferrite LTCC with integrated winding circulator was used as the device under test. Two stacked magnets DH11 on top of DH101 (K\&J magnetics) are employed to provide the external magnetic fields. Three $500 \mathrm{~mm}$ pitch GSG with $2.92 \mathrm{~mm}$ coaxial inputs and two DC probes are used. Two active RF ports are connected to the probes (port 3 of the circulator is terminated in a matched load). The analyzer is Agilent N5244A PNA-X with Option 87 (IMD). The calibration is realized as per instructions for Option 87 IMD measurements (ECal and power meter). Then we performed the swept-power IMD measurement (intermodulation measurement only). Figure 4.39 lists the details of the measurement.

Although oftentimes ignored, IM3 can actually vary as a function of: the input 
power, the centre frequency, and the spacing of the two tones. Option 87 of the PNA$\mathrm{X}$ allows IM3 measurements to be performed while sweeping any of these parameters. For simplicity, only the input power will be swept in this measurement, the rationale being that the best circulator performance was achieved around a centre frequency of $14.5 \mathrm{GHz}$, and using different $\Delta f$ values won't offer any additional insight into the behaviour of the circulator. Only third order intermodulation measurement at 14.5 $\mathrm{GHz}$ are performed with an average of 64 sweeps. The variation of the frequency is $10 \mathrm{MHz}$. Input test tone power swept from $-20 \mathrm{dBm}$ to $+5 \mathrm{dBm}$, levelled at port 1 and spectrum was measured at port 2 (output). Figure 4.39 lists the details of the measurements.

$\begin{array}{ll}\text { Circulator } & \text { 10-layer ESL40012 ferrite LTCC with internal DC winding } \\ \text { Magnets } & \text { two stacked cylindrical magnets: DH11 on top of a DH101 (the latter contacts the LTCC) } \\ \text { Probes } & \text { Three } 500 \mu \mathrm{m} \text { pitch GSG with } 2.92 \mathrm{~mm} \text { coaxial inputs, two DC probes } \\ \text { Ports } & \text { Two active RF ports (port } 3 \text { of the circulator is terminated in a matched load) } \\ \text { Analyzer } & \text { Agilent N5244A PNA-X with Option } 87 \text { (IMD) } \\ \text { Calibration } & \text { As per instructions for Option } 87 \text { IMD measurements (ECal and power meter) } \\ \text { DC bias } & 0,350 \mathrm{~mA} \text { and } 430 \mathrm{~mA}\end{array}$

Figure 4.39: IP3 measurement details

Swept-Power IMD, 0 mA

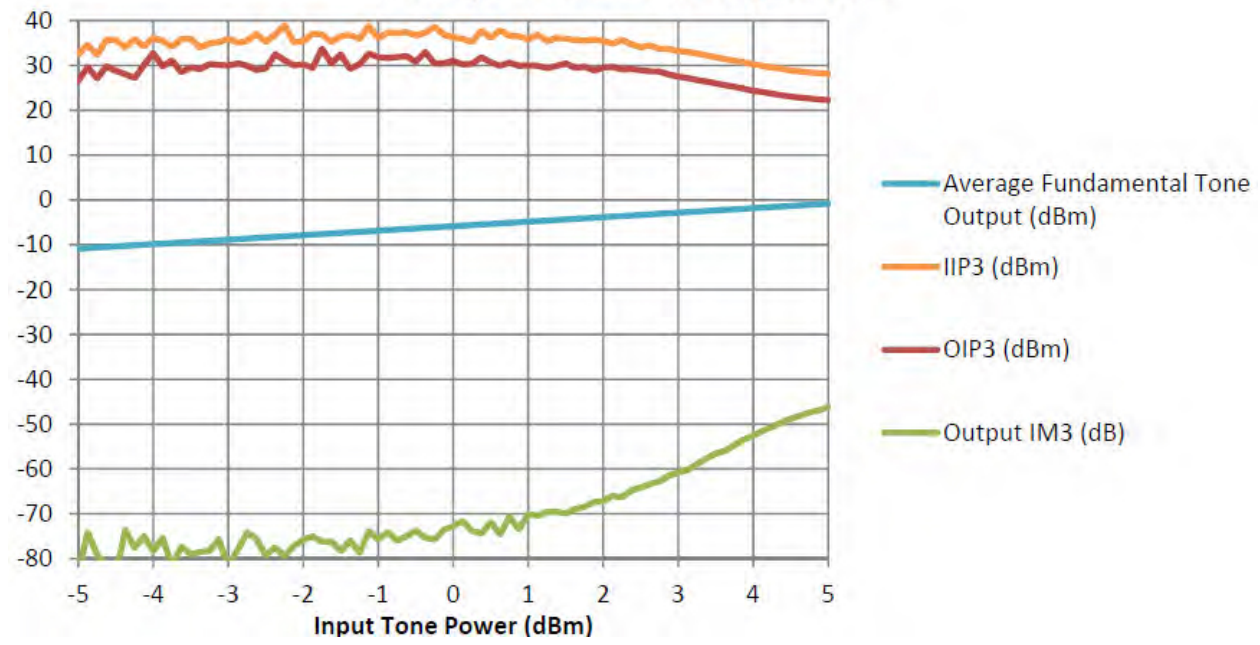

Figure 4.40: Swept-Power IMD Test when current $\mathrm{I}=0 \mathrm{~mA}$ 
Figure 4.41 shows that the IM3 is lost in the noise for Input Tone Powers below approximately $+1 \mathrm{dBm}$. As such, the data should only be trusted above this value. The IIP3 varies from approximately +35 to $+28 \mathrm{dBm}$ for input tone powers between +2 and $+5 \mathrm{dBm}$. There is a clear downward trend, indicating more nonlinearity (decrease of IP3) as the input power is increased. It appears that the value is levellingoff at $+5 \mathrm{dBm}$, but we would need more input power to verify if this is true or not. However, input power $+5 \mathrm{dBm}$ is the maximum power available of the equipment. The OIP3 is higher than the IIP3 because the output power is lower than the input lower due to the losses in the device.

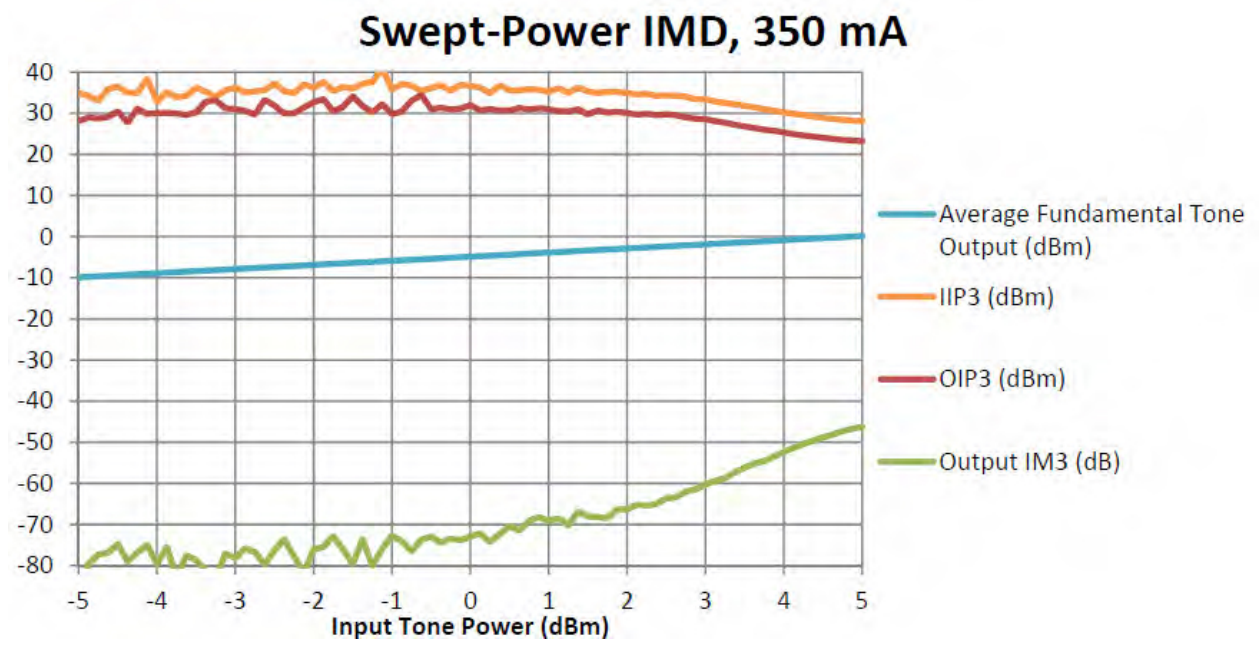

Figure 4.41: Swept-Power IMD Test when current I=350 mA

The results in Figures 4.41 and 4.42 are similar when a dc bias of $350 \mathrm{~mA}$ or 430 $\mathrm{mA}$ is applied, respectively: the IIP3 varies from approximately +35 to $+28 \mathrm{dBm}$ for input tone powers between +2 and $+5 \mathrm{dBm}$. Therefore, the IP3 is about $+30 \mathrm{dBm}$ overall and the P1dB compression point is about $20 \mathrm{dBm}$. 


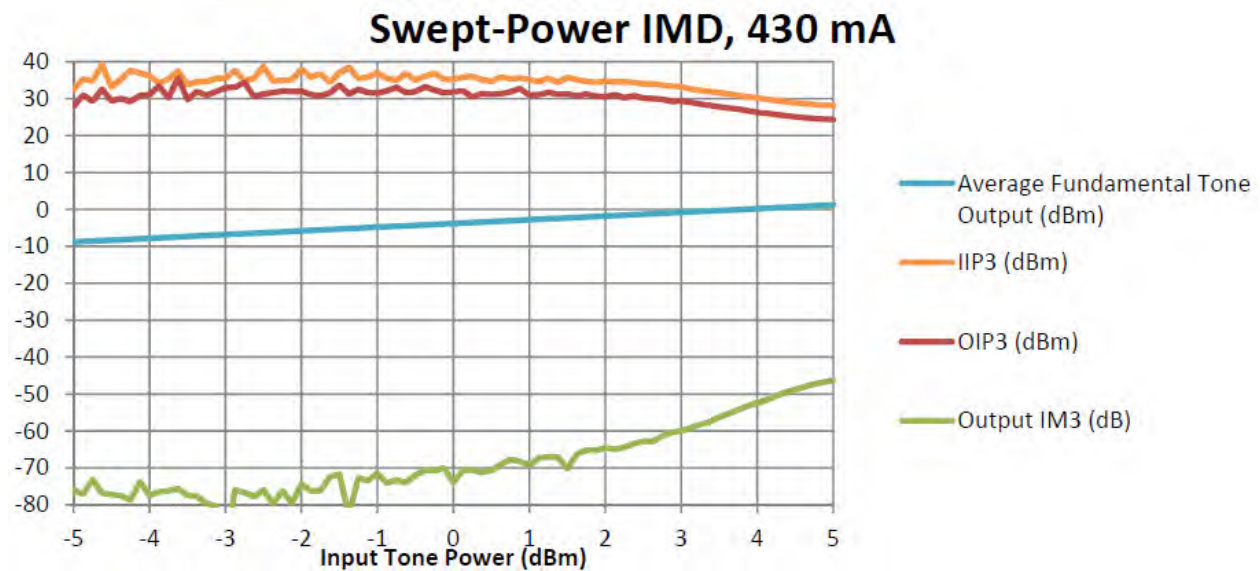

Figure 4.42: Swept-Power IMD Test when current I=430 mA

\subsubsection{Comparison and conclusion}

The table below shows the main performance of different circulators realized by ferrite LTCC and conventional LTCC technology.

\begin{tabular}{llllllll} 
& $\mathrm{F}(\mathrm{GHz})$ & $\mathrm{IL}(\mathrm{dB})$ & $\mathrm{ISO}(\mathrm{dB})$ & $\mathrm{RL}(\mathrm{dB})$ & $\mathrm{P1}(\mathrm{dBm})$ & Tech & Year \\
\hline Our circulator only with current & 14 & 3 & 8 & 20 & NA & Ferrite LTCC & 2015 \\
Our circulator with magnets & 14 & 1.6 & 23 & 22 & 20 & Ferrite LTCC & 2015 \\
Circulator by T. Jensen & 7.4 & 1.7 & 25 & 25 & NA & LTCC & 2011 \\
\hline Circulator by Jianhua Ji & 15 & 0.84 & 26.7 & NA & NA & LTCC & 2009 \\
Circulator by Raymond van Dijk & 5.4 & 0.5 & 22 & NA & 45 & LTCC & 2014 \\
\hline Circulator by Raymond van Dijk & 12 & 1.25 & 25 & NA & 43 & LTCC & 2014
\end{tabular}

Figure 4.43: Comparison of circulators realized by ferrite LTCC and conventional LTCC technology

When only current is used in our circulator, the device has an insertion loss $3 \mathrm{~dB}$ but the isolation is only $8 \mathrm{~dB}$, so it is hard to call it a circulator. When external magnets are applied, the circulator has a better performance leading to an insertion loss about $1.6 \mathrm{~dB}$ and an isolation better than $20 \mathrm{~dB}$. When it is compared with other circulators, especially the circulator at $15 \mathrm{GHz}$ by Jianhua JI, the insertion loss is less competitive. However, our circulators are approaching the performance of the 
circulators realized by co-firing ferrite and conventional LTCC tapes. There are a number of ferrites which are compatible (shrink rate) with the conventional LTCC ceramic substrate. Designers have the freedom in ferrite selection and have taken the losses, working frequency, power requirement etc into account at that stage. However, there are few ferrite LTCC substrate suppliers in the market now and the ESL 40012 is sensitive to the temperature which limits our circulator's power capability. In the future, improved ferrite LTCC materials will lead to a competitive circulator's performance. Moreover, the main advantage of the ferrite LTCC technology over LTCC technology is that it allows to make stacking structures. In the later section, we will present a wide-band stacking circulator.

\subsection{Coplanar ferrite LTCC circulators}

\subsubsection{Introduction}

Previous section has already presented coplanar circulators, which can be easily fabricated at a low cost by using a lithography process. In our laboratory, several coplanar circulators with different operating frequencies are designed, fabricated and measured. This work demonstrates the feasibility of coplanar circulators based on ferrite LTCC and the maximum working frequency at which circulators can operate.

\subsubsection{Design of coplanar circulators}

The same ferrite LTCC tape ESL 40012 is used to design the coplanar circulators, but only 5 layers of tapes are employed. A coplanar circulator consists of a center conductor and metal grounds at the same level, connected to three CPW transmission line. Figure 4.44 [17] shows the top view of a conventional coplanar circulator design. 


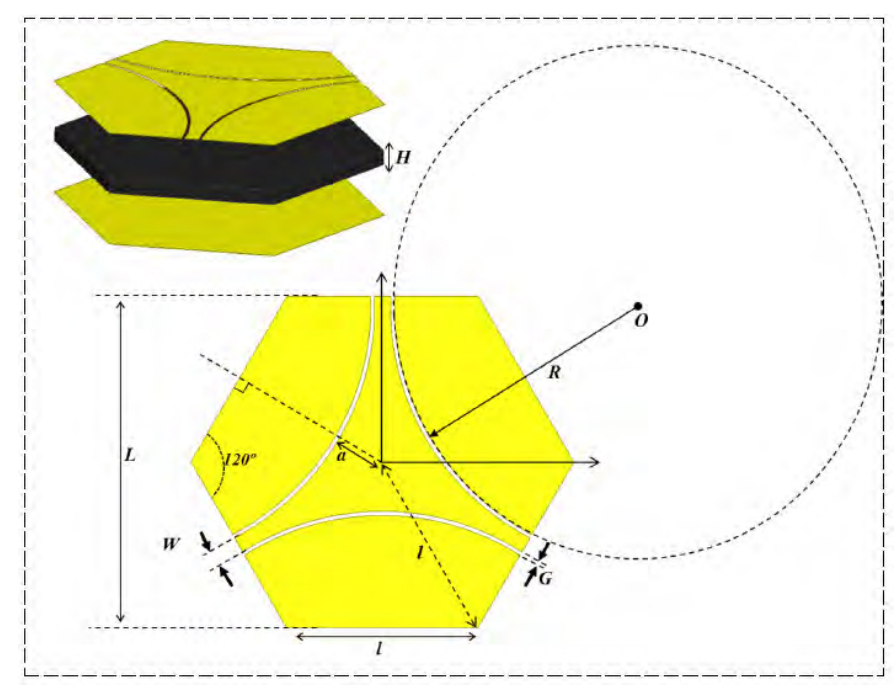

Figure 4.44: Design of a coplanar circulator (top view) [17]

The edge of the center conductor is an arc of a circle having a radius $\mathrm{R}$. The circulator is fed by a CPW transmission line where the width is assumed to be W and the gap is G. The magnetic bias is provided externally by the magnet D101-N52 (K\&J magnetics). Circulations at different frequencies could be found, by optimizing the metal ground size l, W, G and R.

Figure 4.45 shows different fabricated coplanar circulators operating at $18 \mathrm{GHz}$, $20 \mathrm{GHz}, 22 \mathrm{GHz}, 25 \mathrm{GHz}, 27 \mathrm{GHz}$ and $29 \mathrm{GHz}$, respectively. The simulated results have insertion loss better than $1 \mathrm{~dB}$, isolation and return loss higher than $20 \mathrm{~dB}$. Each circulator has two samples. CPW thru is also fabricated for the purpose of calibration.

\subsubsection{Measurements}

Due to a careless manipulation in the fabrication, the circulators have some defects leading to more losses and mismatch. Only the measurements of the circulators at $18 \mathrm{GHz}, 22 \mathrm{GHz}$ and $25 \mathrm{GHz}$ are presented here. 


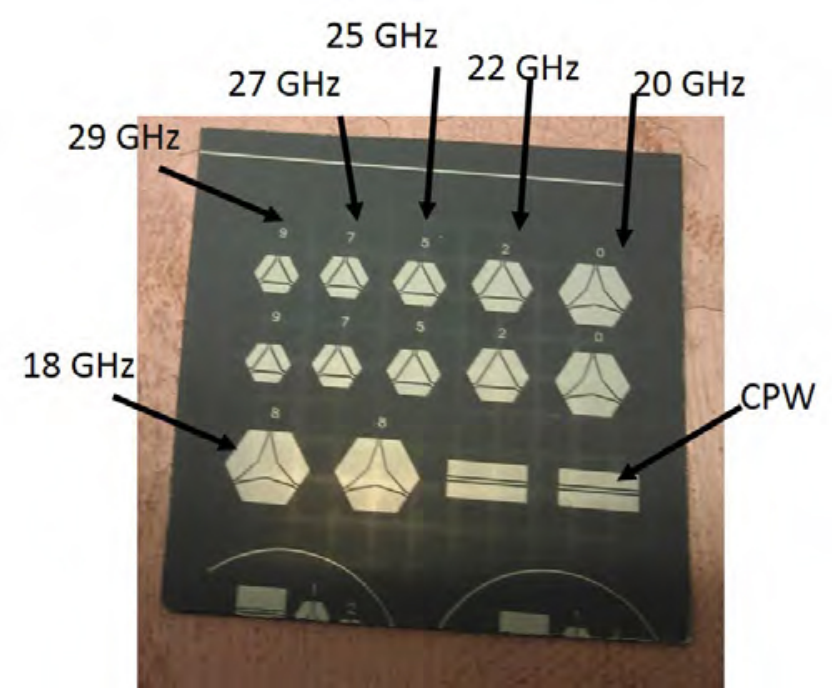

Figure 4.45: Fabricated CPW and coplanar circulators operating at different frequencies

Figure 4.46 shows a circulation around $18 \mathrm{GHz}$. The insertion loss is $4 \mathrm{~dB}$ which is a high loss, however the isolation is $23 \mathrm{~dB}$. The matching is not good due to the defects during the fabrication.

The measured S-parameters of the coplanar circulator working at $22 \mathrm{GHz}$ are shown in Figure 4.47. The matching is better than that of $18 \mathrm{GHz}$ circulator leading to an insertion loss about $3 \mathrm{~dB}$ and the isolation remains higher than $20 \mathrm{~dB}$.

As for the circulator at $27 \mathrm{GHz}$, the transmission and isolation start approaching to each other (toward $-5 \mathrm{~dB}$ ) as shown in Figure 4.48. This measurement demonstrates the difficulty of designing a high frequency circulator.

According to the above measurements, ferrite LTCC ESL40012 could be used to design circulators working from $\mathrm{X}$ band to $\mathrm{K}$ band. Higher frequencies could probably be realized when more powerful magnets are applied, but the characterization of this material over $30 \mathrm{GHz}$ remains to be performed. 


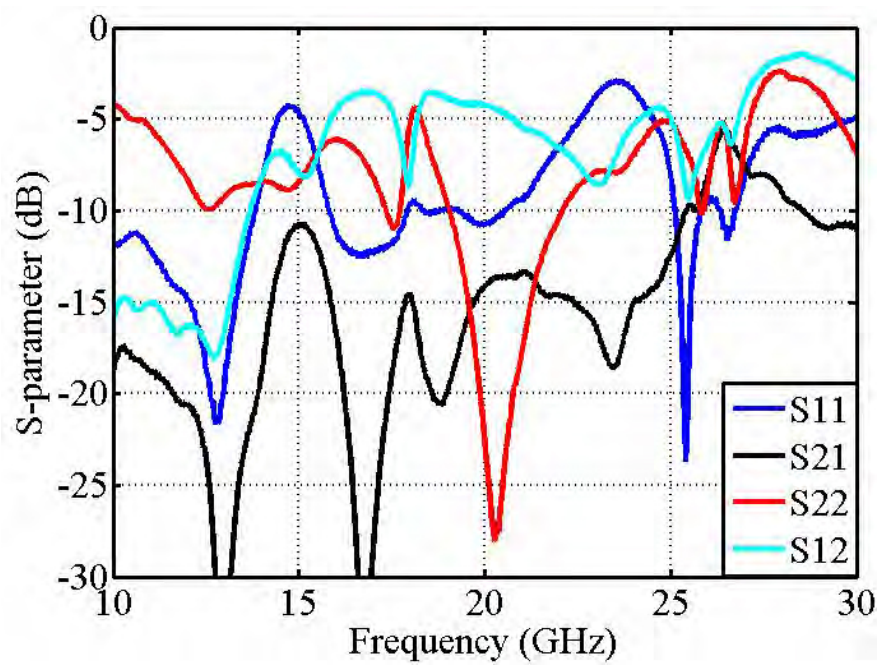

Figure 4.46: Measured S-parameters: coplanar circulator operating at $18 \mathrm{GHz}$

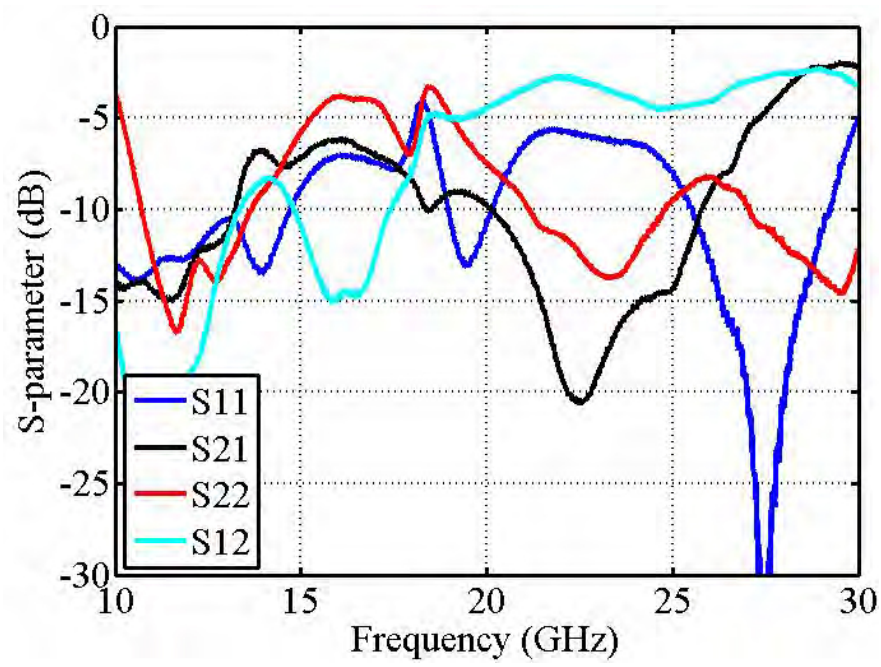

Figure 4.47: Measured S-parameters: coplanar circulator operating at $22 \mathrm{GHz}$ 


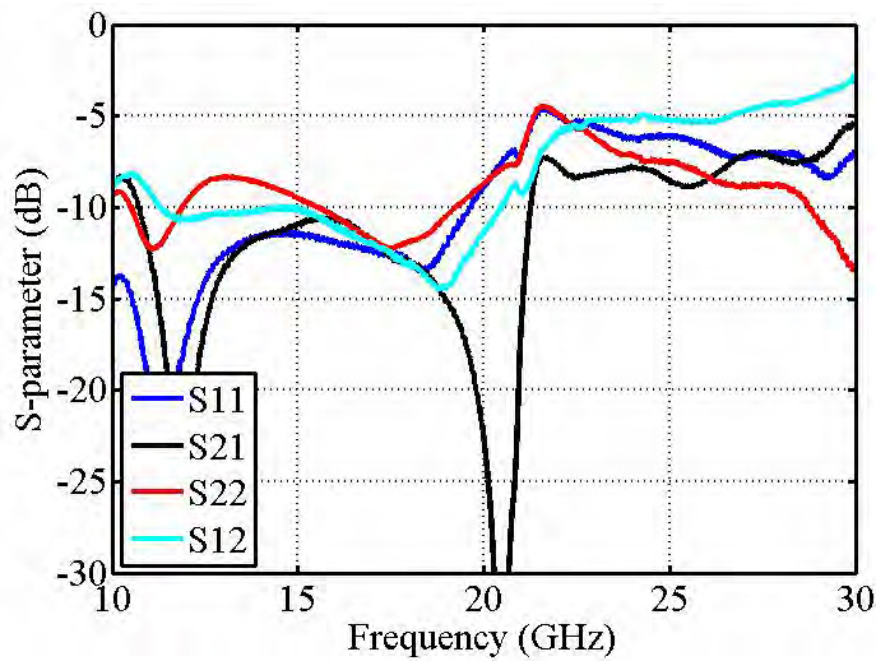

Figure 4.48: Measured S-parameters: coplanar circulator operating at $27 \mathrm{GHz}$

\subsection{Wideband circulator by stacking two circula- tors}

\subsubsection{Introduction}

The conventional circulator with stripline structure is designed to operate at a specific frequency. To expand the bandwidth, we usually work on the shape of the center conductor, or use a mode of "edge-guided" $[59,60,122]$. However, the 3D design capacity of the ferrite LTCC technology can be used to increase the bandwidth. This work presents a new wideband circulator operating at K-band. The design is realized by stacking two circulators (gyrators) with different working frequencies into the ferrite LTCC substrate to expand the overall bandwidth. The obtained bandwidth by simulator Ansys HFSS is $11 \mathrm{GHz}(15.5 \mathrm{GHz}$ to $26.5 \mathrm{GHz}$ ) which covers the K-band. The reflections and isolation are below $-20 \mathrm{~dB}$ while the insertion loss is between 1.5 $\mathrm{dB}$ and $3 \mathrm{~dB}$. 


\subsubsection{Theory and design}

A stripline circulator is chosen because of the two metal ground planes that avoid the external environment $[5,37]$. The circulator design uses ten layers of ESL 40012 ferrite LTCC, where each layer has a thickness of $110 \mu \mathrm{m}$. The electromagnetic properties of this ferrite have already been presented in previous sections.

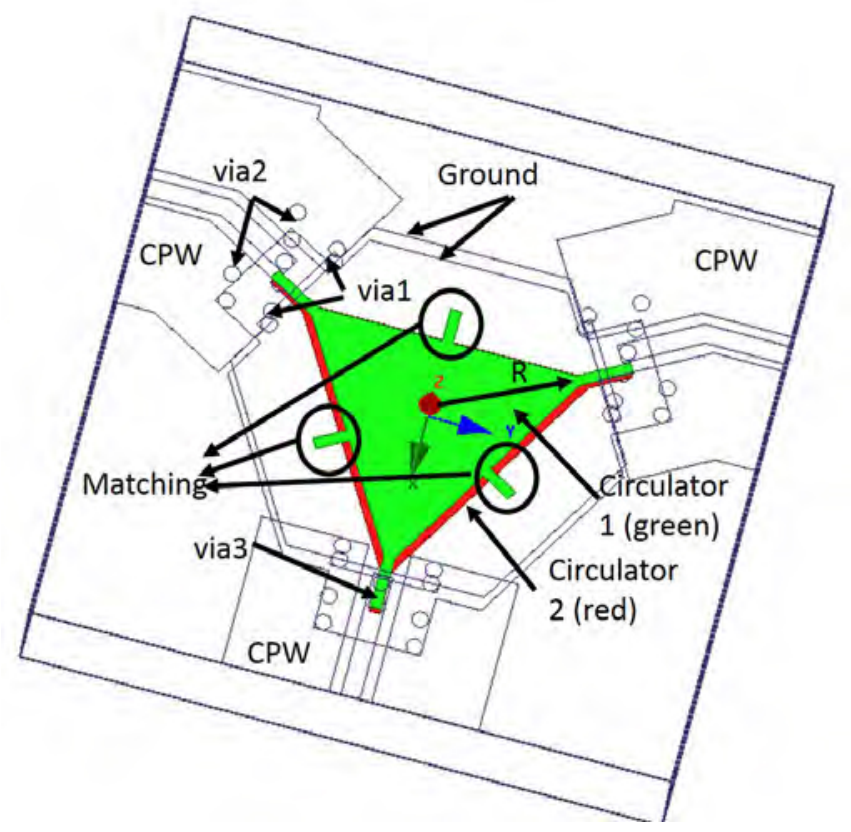

Figure 4.49a: Design of a wideband circulator by stacking two circulators

The design of this two stacking circulator is shown in Figure 4.49a. It is realized using a minimum number of layers: the two metal grounds are located above and below the center conductors of two circulators. Figure $4.49 \mathrm{~b}$ shows the ten layers of LTCC ferrite. The additional layers where there are no conductors (layer 1-3 and 8-10) allow a better bias polarization in the ferrite. These layers help make the magnetic field perpendicular to the circulator plane $\left(H_{i}\right.$ follows the direction $\mathrm{Z}$ in Figure 4.49a). The CPW accesses are located on the top layer (layer 10) and allow the connection to the probe station. The external magnet is put below the device. The 
two center conductors have a triangular shape which provides a wide bandwidth [46]. The dimensions of the two center conductors of the circulators are slightly different to further extend the bandwidth. The design also includes the impedance matching (see Figure 4.49a). One of the design challenges is to feed the two stripline circulators at the same time, because they can only be connected on the top layer (layer 10). The transition from CPW lines to stripline is represented in Figure 4.49b, where two vias of type 1 connect the two grounds of CPW to the upper ground of the stripline; similarly for the vias of type 2 , which connect the two grounds of CPW to the lower ground of the stripline; and finally, a via of type 3 connects the signal conductor of CPW to the two circulators' center conductors. The fact that all ground planes are connected by vias prevents the excitation of parasitic modes. As there is no metal ground between the two center conductors, the two circulators are coupled. The equivalent circulator has a total dimension $(\mathrm{L} * \mathrm{~W} * \mathrm{H}) 8 \mathrm{~mm} * 8 \mathrm{~mm} * 1.1 \mathrm{~mm}$.

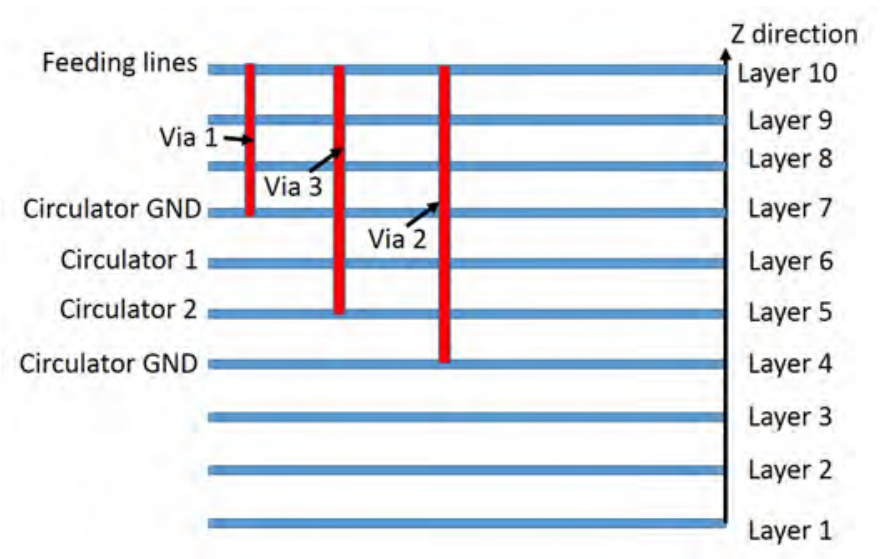

Figure 4.49b: Ferrite LTCC layers configuration

The external magnet ( D42B-N52 from K\&J magnetics), produces a surface field about 5233 Gauss and has a residual flux density $B r_{\max }=14800$ Gauss. Its diameter is $6.35 \mathrm{~mm}(1 / 4 \mathrm{inch})$ and the height is $3.175 \mathrm{~mm}(1 / 8 \mathrm{inchs})$. The magnet is placed below the substrate. A simulation in Ansys Maxwell 3D is performed to find the 
distribution of the internal field in the circulator structure (see Figure 4.50a).

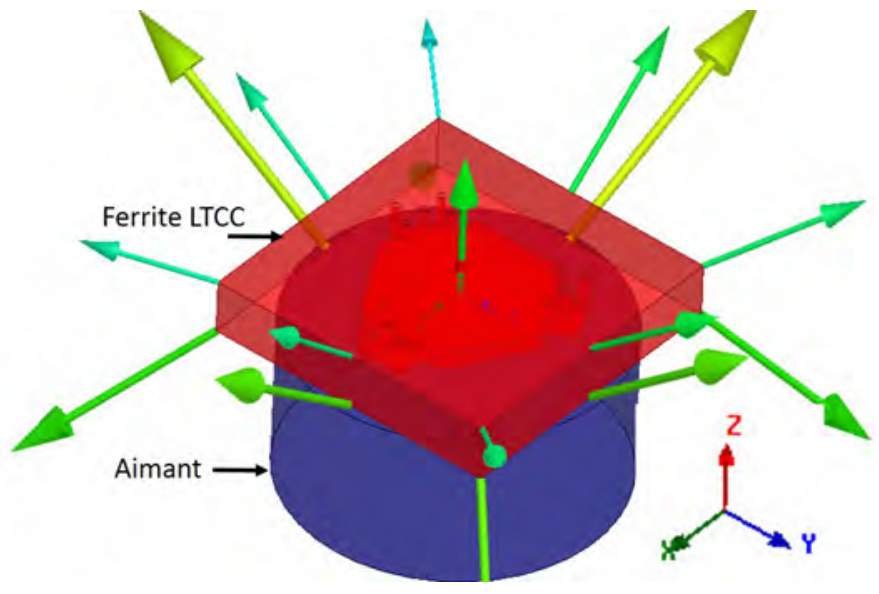

Figure 4.50a: Distribution of magnetic field $H_{i}$ in ferrite LTCC

Figure $4.50 \mathrm{~b}$ shows the internal field $B_{i}$ along the axis $\vec{z}$. According to this plot, the magnetic field mainly follows the $\mathrm{z}$-direction as we expected. The magnetic field strength between the layer 4 and 7 (where there are two cores of the circulators) is greater than $M_{s}$ which means that the magnets could saturate the ferrite material [15].

\subsubsection{Simulation}

Firstly, an approximate simulation (the program in Matlab described before) is performed using the mathematical calculations proposed by Bosma [122]. Although this design is not a traditional stripline circulator, the program still gives an approximate dimension of the center conductor which favors the optimization in Ansys HFSS. Matlab shows a possible circulation at K-band with a radius $R_{c i r}=1.385 \mathrm{~mm}$ (which is the $\mathrm{R}$ in Figure 4.49a).

Although the bias field is nearly uniform, any form of ferrite material has a nonuniform demagnetizing field, resulting in a non-uniform internal field in the ferrite (see Figure 4.50a). In practice, a uniform bias field is difficult to achieve. To model a 


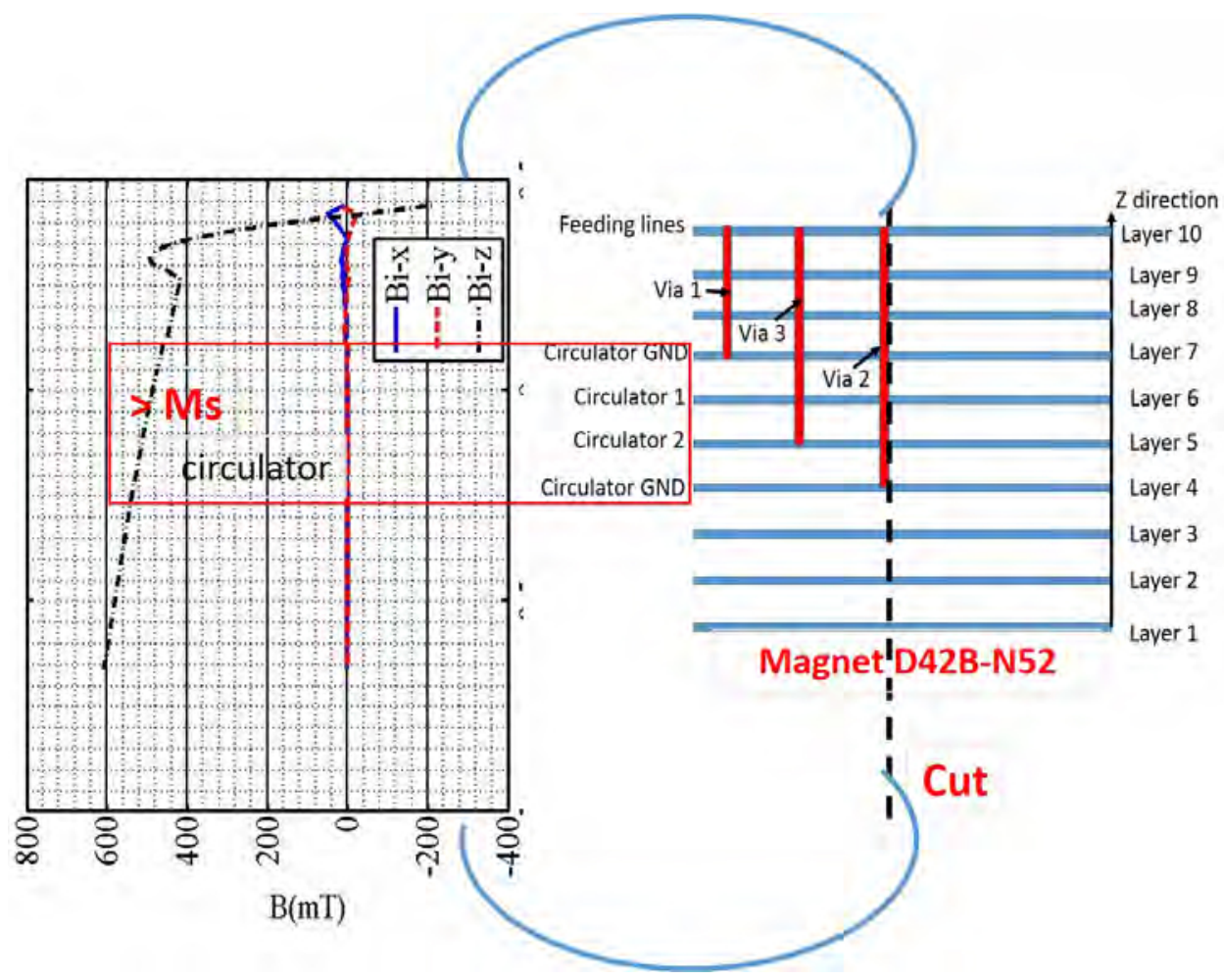

Figure 4.50b: Internal bias field $B_{i}$ in terms of Z

ferrite polarized by a static magnetic field, we need to know the value and the direction of the bias field at any point in the structure. In this design, in order to account for this non-uniform dc bias field, we use the "non-uniform magnetic bias" in Ansys HFSS software which calls Maxwell 3D to generate a solution of the non-uniform magnetostatic magnetic fields. Once the dc simulation is performed, the distribution of the internal magnetic field can be imported into ANSYS HFSS automatically and then be used to perform a rigorous simulation.

We optimize the dimension of each circulator to obtain a wide bandwidth at $\mathrm{K}$ band. The final simulations lead to $R_{1}=1.5 \mathrm{~mm}$ and $R_{2}=1.6 \mathrm{~mm}$ for circulator 1 and 2 , respectively, which is in agreement with the analytical simulation.

Figure 4.51 shows that the circulator has an optimized bandwidth of $11 \mathrm{GHz}$ (15.5-26.5 GHz) which covers the $\mathrm{K}$ band. The reflections and isolation are higher 


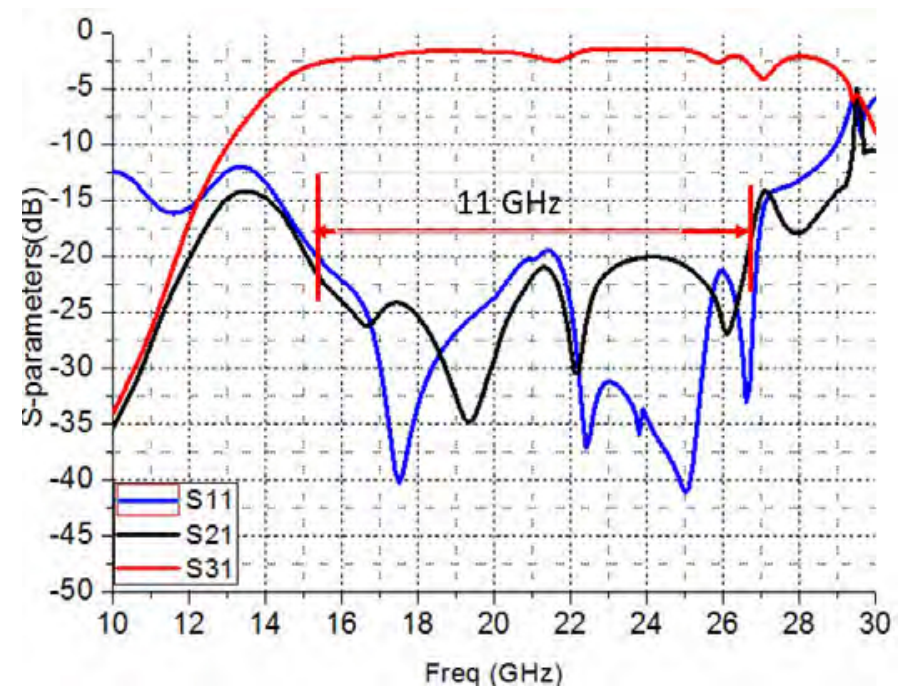

Figure 4.51: S-parameters by co-simulation in HFSS and Maxwell 3D

Table 4.1: Compare of circulators realized with LTCC technology

\begin{tabular}{|c|c|c|c|c|}
\hline $\mathrm{F}(\mathrm{GHz})$ & Bandwidth $(\mathrm{GHz})$ & $\mathrm{IL}(\mathrm{dB})$ & ISO & Reference \\
\hline 7.4 & 0.1 & 1.7 & 30 & {$[24]$} \\
\hline $15-17$ & 2 & $<1$ & 25 & {$[23]$} \\
\hline $11.5-13.5$ & 2 & $<1$ & $>22$ & {$[23]$} \\
\hline $15.5-26.5$ & 11 & $1.5-3$ & $>20$ & this design \\
\hline
\end{tabular}

than $20 \mathrm{~dB}$ and the insertion loss is between $1.5 \mathrm{~dB}$ and $3 \mathrm{~dB}$. Table 4.1 shows the performance of different circulators realized with LTCC technology. Our circulator has an impressing wide-band performance compared with the other three cited circulators, but the insertion loss is higher due to the magnetic material losses $(\Delta H=$ 250 Oe).

\subsection{Conclusion}

In this chapter, a CPW transmission line is measured to have a first impression of the ferrite LTCC device. Then an edge-guided circulator with external magnets is realized 
with a full ferrite LTCC tape without any other ferrites or dielectric materials. At the operating frequency of $15 \mathrm{GHz}$, the transmission is approximately $-1.8 \mathrm{~dB}$, the isolation is $-20 \mathrm{~dB}$ and the reflection is better than $-20 \mathrm{~dB}$. This work demonstrates that circulators and isolators can be integrated into the ferrite LTCC substrate and has shown the potentiality as a candidate for System-in-Package(SiP).

Then a study of a novel circulator with integrated winding is presented, for the first time. The device exhibits a multifunctional device controlled by the winding. When the windings are not energized by the current, the material is not magnetized, so this device operates as a power divider. Each port has a power of $-5 \mathrm{~dB}$ with a return loss better than $20 \mathrm{~dB}$ at $14.8 \mathrm{GHz}$. When the windings are energized by a current, this device operates as a circulator. In addition, the circulator's circulation direction can be changed by switching the feeding current's direction. With a current of $300 \mathrm{~mA}$, the circulator shows a transmission of $-3 \mathrm{~dB}$, an isolation of $-8 \mathrm{~dB}$ and the return loss better than $-20 \mathrm{~dB}$ at $14.2 \mathrm{GHz}$. With an external magnet, the material gets a stronger internal field which improves greatly the performance: transmission $-1.8 \mathrm{~dB}$, isolation $-18 \mathrm{~dB}$ and return loss better than $-20 \mathrm{~dB}$ at $14.8 \mathrm{GHz}$. When external magnets and current are both applied, adjustment of the internal $\mathrm{H}$ field by changing the current is possible to get a better result: transmission $-1.6 \mathrm{~dB}$ with isolation and return loss both better than $20 \mathrm{~dB}$ at $14.2 \mathrm{GHz}$. The circulator's working frequency variation is $0.6 \mathrm{GHz}$. This is caused firstly by the change of internal magnetization $\mathrm{M}$ when current is less than $120 \mathrm{~mA}$, then the heat due to the winding increases the ferrite's $\mu_{e f f}$ leading to more frequency shifting. This novel circulator's performance is limited by the ferrite LTCC material. There are currently few commercially available ferrite LTCC materials, none specifically for microwave. A fully magnet-free, frequency tunable, and multifunctional circulator controlled by the winding is expected, as better materials with temperature stability, low-loss and 
more layer-capacity become available.

Finally a design of a wideband circulator by stacking two circulators in ferrite LTCC is described. It is possible to integrate circulators with different operating frequencies into ferrite LTCC to create dual-band or tri-band circulators or combine their effects to achieve a broadband circulator. Although losses are still too high, simulated performance is significant for a very wide fractional bandwidth (50\%). 


\section{Chapter 5}

\section{Summary, conclusions and perspectives}

\section{$5.1 \quad$ Summary}

This thesis studied rf non-reciprocal components (circulators) based on ferrite LTCC. We investigated an original circulator design where the circulator is fully embedded in ferrite LTCC, studied the performance of a ferrite LTCC circulator with an integrated winding and investigated a wide-band circulator based on the ferrite LTCC substrate. The circulator designs were realized at the University of Saint Etienne France, Laboratory Hubert Curien UMR CNRS 5516, Carleton University Canada and Royal Military College Canada.

The thesis presented the design procedure of a ferrite LTCC circulator with an integrated winding as follows: structure of the circulator $\Rightarrow$ choice of magnetic material $\Rightarrow$ choice of operating point $\Rightarrow$ DC simulation of the winding $\Rightarrow$ Matlab simulation $\Rightarrow \mathrm{EM}$ simulation $\Rightarrow$ fabrication and measurements $\Rightarrow$ retro-analysis.

The structure of the circulator is described in Chapter 2. In our design, the junction circulator is employed because it has been studied by many researchers and widely employed in microwave applications, such as radars. In the circulator, we also have different planar transmission lines, such as stripline, microstrip and coplanar 
waveguide. Since we will integrate a winding into the circulator, we must protect it from the interface of the winding. Therefore, stripline is chosen because its two metal grounds provide rf shielding against the winding while simultaneously allowing the magneto-static bias field into the ferrite core.

The magnetic material and the choice of the operating point are discussed in Chapter 3. The main difference between ferrite materials and dielectric materials is their permeability. For ferrites, as an example, the permeability is a tensor instead of a constant. Circulators commonly work on saturated ferrites and Polder's equations are used to calculate the tensor elements. If the ferrite is partially magnetized, there are a number of theories to model the permeability tensor. In this thesis, we used the Green and Sandy model, which is obtained based on the measurements of the experimental characterization cells. It is valid when the operating frequency is higher than the resonance frequency. This model is easier to implement than the GPT model. Moreover, this model has been implemented to analyze a patch antenna based on the partially magnetized ferrite LTCC ESL 40012 substrate and proven to be a good approximate model. The magnetization frequency is $11.2 \mathrm{GHz}$ when $\mu_{0} M_{s}=0.4 \mathrm{~T}$, so an operating frequency higher than $14 \mathrm{GHz}$ is chosen to avoid low-field losses. We operate our circulators in the "low $H_{i}$ zone" which requires a weak $\mathrm{H}$ field. If the ferrite is partially magnetized, the tensor element $\mu$ or $\kappa$ is a function of the internal magnetization $\mathrm{M}$ and they are in proportion. The $\mu_{\text {eff }}$ is also a function of the internal magnetization $\mathrm{M}$, which could be used to analyze the circulator's operating frequency variation.

In Chapter 4, we presented the design, simulation and measurement of the winding and the circulator with the integrated winding. Ten ferrite LTCC layers were used and the space priority is given to the DC winding. The stripline circulator only occupies three of the middle layers (5-7) and the top layer is reserved for the connections, so all 
the other layers are used to design the winding. Therefore, we have four coils below the circulator on layers 1-4 and two coils above the circulator on layers 8 and 9 . On each layer of the winding, a hexagonal planar coil is chosen because it approximates a circular one. Each coil consists of 5 turns of a $0.1-\mathrm{mm}$ wide conductor with a $0.1-\mathrm{mm}$ spacing, both are the minimum values allowed in the fabrication process. The inner coil turn has a side-to-side width of $0.4 \mathrm{~mm}$ whereas the outer turn has a side-to-side width of $2.2 \mathrm{~mm}$. $150-\mu \mathrm{m}$ diameter vias are used to connect the coils and DC current in the winding is supplied by two de pads on the top layer.

Next, we performed the dc simulation in Maxwell 3D. The internal magnetic field is approximately $200 \mathrm{mT}$ in the core of the circulator when the applied current is 300 mA. The magneto-static field tends to create a closed dipole field around the upper and lower coils because the ferrite-air boundary limits the field in the ferrite material and the xy flux paths are thin and tend to saturate first. On layer 9, the de magnetic field is in the plane xy as we expected and on the layer of the center conductor of the circulator, the dc magnetic field mainly follows the z-direction for a radius of $0.6 \mathrm{~mm}$ and then it turns to the plane xy. Based on the theory of Bosma, we performed Sparameter simulations when the internal magnetization $\mu \mathrm{M}$ is $200 \mathrm{mT}$. A circulation at $14 \mathrm{GHz}$ is found and the radius of the circulator is found to be about $1.79 \mathrm{~mm}$. Matlab uses Green and Sandy's model, so the circulator's operating frequency f is a function of $\mu_{e f f}$. However, $\mu_{e f f}$ depends on then internal magnetization M, so f is a function of $\mathrm{M}$ as well. Therefore, we performed several S-parameter simulations with different internal magnetization $M$. When $M=0$, we see the superposition of the curve S12 and S21 which means that the circulator operates as a power divider. When we increase the value for $\mathrm{M}$, we separate these two curves, creating the circulator along with a frequency variation. According to these simulations, the circulator's operating frequency is inversely proportional to the value of $\mathrm{M}$. 
We then designed the circulator in HFSS. HFSS uses Polder's equations for saturated ferrites. In order to perform an approximate simulation in HFSS, seven parts of ferrite are defined in HFSS whose $M_{s}$ are replaced by the M because the ferrite is not fully saturated. The part 7 follows the z-direction with an average value about $199 \mathrm{mT}$ and the parts 1-6 have radial directions with an average value about $132 \mathrm{mT}$. We performed the simulation in HFSS and compared the results with Matlab simulations. Both resulted in a circulation at about $14 \mathrm{GHz}$. The optimized radius of the circulator in HFSS is $1.73 \mathrm{~mm}$ which is in agreement with the Matlab simulation 1.79 $\mathrm{mm}$. Matlab gave a better insertion loss. This comparison tells that our approximate model works to some extent.

Following the simulations we fabricated the the circulators with a price of 30,000 euros for a panel of $5 \mathrm{~cm} * 5 \mathrm{~cm}$. We then performed the S-parameter measurements with an applied current $300 \mathrm{~mA}$ and compared the results with HFSS simulations. HFSS predicted a good result for reflections, but was not accurate for the insertion loss and isolation due to the inaccurate ferrite model in HFSS. However, the operating frequency $14 \mathrm{GHz}$ is well predicted. Since the ferrite is not saturated, we performed S-parameter simulations with external magnets. The presence of the magnets greatly improved the measured performance, leading to an insertion loss $1.6 \mathrm{~dB}$ and an isolation over $20 \mathrm{~dB}$. However, we observed a frequency variation about $0.6 \mathrm{GHz}$. The variation of the circulator's working frequency is $0.6 \mathrm{GHz}$. This is achieved firstly by the change of internal magnetization M when current is less than $120 \mathrm{~mA}$. Subsequent heat in the substrate due to the winding current introduces more frequency shifting. Later, we performed the retro-analysis using CST studio where Green and Sandy's model was entered. The simulation in CST gave a close result for the insertion loss and isolation. However the refection result was not accurate, probably because we used a simplified and uniform bias field in CST. A frequency variation about $0.2 \mathrm{GHz}$ 
is observed when $\mu \mathrm{M}$ goes from 0 to $0.2 \mathrm{~T}$. Also we measured the IP3 to be about 30 dBm leading to a P1dB about 20dBm. Then we compared our ferrite LTCC circulator with other LTCC circulators. The comparison shows that our circulators approach the performance of circulators realized by co-firing ferrite and LTCC tapes. Since there are a number of ferrite materials which could be co-fired with LTCC substrate, designers have the freedom to choose the right ferrite (losses, power, $M_{s}$ etc) for their devices. However, there are few commercial ferrite LTCC substrates and ESL 40012 is sensitive to the temperature which limits the power capability. Later we tested several coplanar circulators to find the maximum operating frequency that the material can support.

Finally a design of a wideband circulator by stacking two circulators in ferrite LTCC was described. It is possible to integrate circulators with different operating frequencies into ferrite LTCC to create dual-band, tri-band circulators or combine their bandwidths to achieve a broadband circulator. Although the insertion loss is about $1.5 \mathrm{~dB}$, the bandwidth is really impressive, about $11 \mathrm{GHz}$.

\subsection{Conclusions}

A study of a novel circulator with an integrated winding based on ferrite LTCC was performed. The self-biasing which can be achieved by this method is less reliable than the use of external magnets. The circulator's performance is less competitive than that of circulators based on conventional LTCC. This is primarily due to limitations of the ferrite LTCC substrate which was available. However, a fully magnet-free, frequency tunable and multifunctional circulator controlled by the winding is expected, as better materials with temperature stability, low-loss and more layer-capacity become available. In addition, the thesis demonstrates that a wide-band circulator can 
be designed by stacking two circulators with different operating frequencies.

\subsection{Perspectives}

Improved performance of the circulator with integrated winding can be expected when improved commercial ferrite LTCC materials become available, especially with high temperature capability and low losses. Also, an advanced fabrication process with capability for more and/or thinner layers could also contribute to a compact winding design leading to more dc magnetic field in the circulator. The GPT permeability tensor model could be used to improve the circulator design and optimization.

In the future, circulators could be integrated with other rf components such as oscillators, power amplifiers, filters, antennas etc using ferrite LTCC technology to achieve final products. LTCC technology could also offer multi-material modules, where materials with different electrical properties could be combined within the substrate.

In order to increase the internal magnetic field, small magnets could be integrated into the ferrite LTCC substrate creating the bias field from the inside. This would greatly improve the strength of the internal magnetic field, since there would be no demagnetization fields.

We could also expect a "hard" ferrite LTCC substrate to become available which allows a winding to be embedded. By energizing the winding with a pulse of current, a latching switch could be realized based on a circulator.

Dual-band or multi-band circulators realized by stacking several circulators with different frequency bands are possible. Integrated active elements or varactors could be used to perform matching compensation for the impedance variation of the circulator. 


\section{List of References}

[1] I. JQL Electronics, "Curie temperature," official website, 2015.

[2] P. Semiconductors, "Circulators and isolators, unique passive devices," Philips Semiconductors, 1998.

[3] E. Ohm, "A broad-band microwave circulator," IRE Transactions on Microwave Theory and Techniques, vol. 4, no. 4, pp. 210-217, 1956.

[4] S. Ewing and J. Weiss, "Ring circulator theory, design, and performance," IEEE Trans. MTT, vol. 15, no. 11, pp. 623-628, 1967.

[5] H. Bosma, "On stripline Y-circulation at UHF," IEEE Trans. MTT, vol. 12, no. 1 , pp. $61-72,1964$.

[6] H. Dong, J. Smith, and J. Young, "A wide-band, high isolation UHF lumpedelement ferrite circulator," Microwave and Wireless Components Letters, IEEE, vol. 23, no. 6, pp. 294-296, 2013.

[7] J. Wang, A. Yang, Y. Chen, Z. Chen, A. Geiler, S. Gillette, V. G. Harris, and C. Vittoria, "Self biased Y-junction circulator at ku band," Microwave and Wireless Components Letters, IEEE, vol. 21, no. 6, pp. 292-294, 2011.

[8] N. Estep, D. Sounas, and A. Alu, "Magnetic-free, fully integrated, compact microwave circulator using angular-momentum biasing," Antennas and Propagation Society International Symposium (APSURSI), 2014 IEEE, pp. 340-341, 2014.

[9] D. M. Pozar, "Microwave engineering 4th edition," John Wiley and Sons, Inc, 2011.

[10] A. A. Nassiri, "Ferrite material," Microwave Physics and Techniques, 2003. 
[11] MMG, "Magnetic materials background information," University of Birmingham, 2015.

[12] B. Bayard, "Thesis report: Contribution au developpement de composants passifs magnetiques pour l'electronique hyperfrequence," Université Jean Monnet Saint Etienne France, 2000.

[13] R. Center, "Resource center," NDT Course Material, 2015.

[14] T. CERAMICS, "Temex: Microwave ferrite materials," Microwave Ferrites, 2015.

[15] A. Shamim, J. Bray, N. Hojjat, R. A. Elasoued, and D. Baillargeat, "Microwave and magnetostatic characterization of ferrite LTCC for tunable and reconfigurable sip applications," Microwave Symposium, 200\%. IEEE/MTT-S International, pp. 691-694, 2007.

[16] Y. Imanaka, "Multilayered low temperature cofired ceramics (ltcc) technology," Springer, 2005.

[17] A. TCHANGOULIAN, "Thesis report: Utilisation de matériaux composites magnétiques à nanoparticules pour la réalisation de composants passifs non réciproques micro-ondes," Université Jean Monnet Saint Etienne France, 2014.

[18] K. Oshiro, H. Mikami, S. Fujii, T. Tanaka, H. Fujimori, M. Matsuura, and S. Yamamoto, "Fabrication of circulator with coplanar wave guide structure," IEEE Trans. Magnetics, vol. 41, no. 10, pp. 3550-3552, 2005.

[19] T. Wada, R. Nakajima, H. Obiya, T. Ogami, M. Koshino, M. Kawashima, and N. Nakajima, "A miniaturized broadband lumped element circulator for reconfigurable front-end system," Microwave Symposium (IMS), 2014 IEEE MTT-S International, pp. 1-3, 2014.

[20] G. Radosavljevic, L. Zivanov, W. Smetana, A. Maric, M. Unger, and L. Nad, “A wireless embedded resonant pressure sensor fabricated in the standard LTCC technology," IEEE_J_SENSOR, vol. 9, no. 12, pp. 1956-1962, 2009.

[21] K.-L. Wu and Y. Huang, "LTCC technology and its applications in high frequency front end modules," Antennas, Propagation and EM Theory, 2003. ARTICLE. 2003 6th International SYmposium on, pp. 730-734, 2003. 
[22] C. Atzlesberger and W. Smetana, "Evaluation of a centrifugal micropump realized in LTCC-technology," Electronics Technology, 30th International Spring Seminar on, pp. 243-247, 2007.

[23] I. Ii, Y. Li, S. Ruan, I. Zhou, and Y. Fei, "Integrated ltcc transmitter module at ku band," Microwave Technology and Computational Electromagnetics, 2009. ICMTCE. International Conference on, pp. 214-217, 2009.

[24] T. Jensen, V. Krozer, and C. Kjaergaard, "Realisation of microstrip junction circulator using LTCC technology," Electronics Letters, vol. 47, no. 2, pp. 111$113,2011$.

[25] R. van Dijk, G. van der Bent, M. Ashari, and M. McKay, "Circulator integrated in low temperature co-fired ceramics technology," European Microwave Conference 2014 Rome, p. 1544 C 1547, 2014.

[26] F. Ghaffar and A. Shamim, "A ferrite LTCC based dual purpose helical antenna providing bias for tunability," Antennas and Wireless Propagation Letters, IEEE, to be published. Early Access.

[27] A. Shamim, J. R. Bray, N. Hojjat, and L. Roy, "Ferrite ltcc-based antennas for tunable sop applications," IEEE Transactions on Components, Packaging and Manufacturing Technology, vol. 1, no. 7, pp. 999-1006, 2011.

[28] F. A.Ghaffar, J. R. Bray, and A. Shamim, "Theory and design of a tunable antenna on a partially magnetized ferrite LTCC substrate," IEEE Trans. Antennas and Propagation, vol. 62, no. 3, pp. 1238-1245, 2014.

[29] E. Arabi, F. Ghaffar, and A. Shamim, "Tunable bandpass filter based on partially magnetized ferrite LTCC with embedded windings for SoP applications," Microwave and Wireless Components Letters, IEEE, vol. 25, no. 1, pp. 16-18, 2015 .

[30] J. Bray and L. Roy, "Development of a millimeter-wave ferrite-filled antisymmetrically biased rectangular waveguide phase shifter embedded in lowtemperature cofired ceramic," IEEE Trans. MTT, vol. 52, no. 7, pp. 1732-1739, 2004.

[31] J. Adam, G. F. Dionne, E. Schloemann, and S. Stitzer, "Ferrite devices and materials," IEEE Trans. MTT, vol. 50, no. 3, pp. 721-737, 2002. 
[32] Ü. Özgur, Y. Alivov, and H. Morkoç, "Microwave ferrites," Journal of Materials Sciences: Materials in Electronics, pp. 1-169, 2009.

[33] N. Zeina, H. How, C. Vittoria, and R. West, "Self-biasing circulators operating at ka-band utilizing m-type hexagonal ferrites," IEEE Trans. Magnetics, vol. 28, no. 5, pp. 3219-3221, 1992.

[34] C. L. Hogan, "The ferromagnetic faraday effect at microwave frequencies and its applications: The microwave gyrator," vol. 31, no. 1, pp. 1-31, 1952. Bell System Technical Journal.

[35] J. Helszajn and P. Walker, "Operation of high peak power differential phase shift circulators at direct magnetic fields between subsidiary and main resonances," IEEE Trans. MTT, vol. 26, no. 9, pp. 653-658, 1978.

[36] Button and J. Kenneth, "Theoretical analysis of the operation of the fielddisplacement ferrite isolator," IRE Transactions on Microwave Theory and Techniques, vol. 6, no. 3, pp. 303-308, 1958.

[37] H.Bosma, "On the principle of stripline circulation," ARTICLE of the IEE-Part B: Electronic and Communication Engineering, vol. 109, no. 21, pp. 137-146, 1962.

[38] C. Fay and R. Comstock, "Operation of the ferrite junction circulator," IEEE Trans. MTT, vol. 13, no. 1, pp. 15-27, 1965.

[39] Y. S. WU and F. J. Rosenbaum, "Wide-band operation of microstrip circulators," IEEE Trans. MTT, vol. 22, no. 10, pp. 849-856, 1974.

[40] S. Ayter and Y. Ayasli, "The frequency behavior of stripline circulator junctions," IEEE Trans. MTT, vol. 26, no. 3, pp. 197-202, 1978.

[41] J. Helszajn, "Frequency response of quarter-wave coupled reciprocal stripline junctions," IEEE Trans. MTT, vol. 21, no. 8, pp. 533-537, 1973.

[42] E. Schloemann and R. Blight, "Broad-band stripline circulators based on yig and li-ferrite single crystals," IEEE Trans. MTT, vol. 34, no. 12, pp. 1394-1400, 1986.

[43] J. Weiss, N. Watson, and G. Dionne, "New uniaxial-ferrite millimeter-wave junction circulators," Microwave Symposium Digest, 1989., IEEE MTT-S International, pp. 145-148, 1989. 
[44] V. Laur, G. Vérissimo, P. Queffelec, L. A. Farhat, H. Alaaeddine, J.-C. Reihs, E. Laroche, G. Martin, R. Lebourgeois, and J.-P. Ganne, "Modélisation et caractérisation d'un circulateur auto-polarisé en bande millimétrique," XIXèmes Journées Nationales Microondes 3-4-5 Juin 2015 - Bordeaux, 2015.

[45] V. Laur, J. Mattei, and P. Queffelec, "Conception et réalisation d'un circulateur en technologie siw à base de nanocomposites ferrimagnétiques," XIXèmes Journées Nationales Microondes 3-4-5 Juin 2015 - Bordeaux, 2015.

[46] T. Boyadjian, "Thesis report: Étude et realisation dun circulateur hyperfréquence à nano particules magnétiques orientées dans la bande 40-60 ghz," Université Jean Monnet Saint Etienne France, 2011.

[47] L. G. Maloratsky, "Passive rf and microwave integrated circuits," Elsevier Inc, 2004.

[48] I. MECA Electronics, "isolators," official website, 2015.

[49] Wikipedia, "Curie temperature," Wikipedia, 2015.

[50] B. Dillon and A. Gibson, "Finite element analysis of high power differential phase shift circulators," Antennas and Propagation, 2001. Eleventh International Conference on (IEE Conf. Publ. No. 480), vol. 2, pp. 871-875, 2001.

[51] P. J. Allen, "The turnstile circulator," IRE Transactions on Microwave Theory and Techniques, vol. 4, no. 4, pp. 223-227, 1956.

[52] M. Shalaby, M. Peccianti, Y. Ozturk, and R. Morandotti, "A non-reciprocal broadband terahertz isolator," Lasers and Electro-Optics (CLEO), 2013 Conference on, pp. 1-2, 2013.

[53] S. Ju, S. Jeong, Y. Kim, P. Watekar, and W.-T. Han, "Demonstration of alloptical fiber isolator based on a cdse quantum dots doped optical fiber operating at 660 nm," Journal of Lightwave Technology, vol. 31, no. 16, pp. 2793-2798, 2013.

[54] J. Weiss, "Circulator synthesis," IEEE Trans. MTT, vol. 13, no. 1, pp. 38-44, 1965.

[55] W. Siekanowicz, R. Paglione, and T. E. Walsh, "A latching ring-and-post ferrite waveguide circulator," IEEE Trans. MTT, vol. 18, no. 4, pp. 212-216, 1970. 
[56] J. Helszajn, "A ferrite ring stripline junction circulator," Radio and Electronic Engineer, vol. 32, no. 1, pp. 55-60, 1966.

[57] U. MILANO, J. H. SAUNDERS, and J. L. DAVIS, "A Y-junction strip-line circulator," IRE Transactions on Microwave Theory and Techniques, vol. 8, no. 3, pp. 346-351, 1960.

[58] H. Carlin, "Non-reciprocal network theory applied to ferrite microwave devices," ARTICLE of the IEE-Part B: Radio and Electronic Engineering, vol. 104, no. 6, pp. 316-319, 1957.

[59] P. D. Santis and F. Pucci, "The edge-guided-wave circulator (short papers)," IEEE Trans. MTT, vol. 23, no. 6, pp. 516-519, 1975.

[60] P. Santis and F.Pucci, "Symmetrical four-port edge-guided wave circulators," IEEE Trans. MTT, vol. 24, no. 1, pp. 10-18, 1976.

[61] K. Araki, T. Koyama, and Y. Naito, "New edge guided mode devices," $M i$ crowave Symposium, 1975 IEEE-MTT-S International, pp. 250-253, 1975.

[62] M. Hines, "Reciprocal and nonreciprocal modes of propagation in ferrite stripline and microstrip devices," IEEE Trans. MTT, vol. 19, no. 5, pp. 442-451, 1971.

[63] J. Helszajn, "The lumped element circulator," The Stripline Circulators:Theory and Practice, pp. 221-237, 2008.

[64] Y. Konishi, "Lumped element Y circulator," IEEE Trans. MTT, vol. 13, no. 6, pp. $852-864,1965$.

[65] C. Christensen, "Synthesis of lumped element ferrite circulators," Microwave Conference, 1971. 2nd European, vol. 2, pp. 1-4, 1971.

[66] T. Wada, R. Nakajima, H. Obiya, T. Ogami, and M. Koshino, "A miniaturized broadband lumped element circulator for reconfigurable front-end system," Microwave Symposium (IMS), 2014 IEEE MTT-S International, pp. 1-3, 2014.

[67] Y. AKAIWA and T. OKAZAK, "An application of a hexagonal ferrite to a millimeter-wave Y circulator," IEEE Trans. Magnetics, vol. 10, no. 2, pp. 374378, 1974. 
[68] E. Schloemann, "Circulators for microwave and millimeter-wave integrated circuits," Proceedings of the IEEE, vol. 76, no. 2, pp. 188-200, 1988.

[69] D. L. Sounas, C. Caloz, and A. Al, "Giant non-reciprocity at the subwavelength scale using angular momentum-biased metamaterials," Nat. Commun, 2013.

[70] I. Korn, "OQPSK and MSK systems with bandlimiting filters in transmitter and receiver and various detector filters," IEE ARTICLE F Communications, Radar and Signal Processing, vol. 127, no. 6, pp. 439-447, 1980.

[71] W. Campbell and T. Parks, "Optimal design of transmitter and receiver filters with mixed performance objectives," Acoustics, Speech, and Signal Processing, 1996. ICASSP-96. Conference ARTICLE., 1996 IEEE International Conference on, vol. 3, pp. 1527-1529, 1996.

[72] Wikipedia, "Isolator(microwave)," Wikipedia, 2015.

[73] B. Bayard, B. Sauviac, and D. Vincent, "Encyclopedia of rf and microwave engineering," John Wiley and Sons, Inc, no. 6, 2005.

[74] E. Salonen and P. Jokela, "The effects of dry snow on reflector antennas," Antennas and Propagation, 1991. ICAP 91., Seventh International Conference on (IEE), pp. 17-20, 1991.

[75] M. Microwave, "Overview of phase shifters and switches," MESL Microwave, 2015.

[76] I. Harris and A. Williams, "Magnetic materials," School of Metallurgy and Materials, University of Birmingham, 2005.

[77] Wikipedia, "Wikipedia paramagnetism," Wikipedia, 2015.

[78] Wikipedia, "Wikipedia ferromagnetism," Wikipedia, 2015.

[79] D. S. Mathew and R.-S. Juang, "An overview of magnetism of spinel nanoferrite particles and a study of chromium substituted zn-mn ferrites nanostructures via sol-gel method," Nanoscience, Engineering and Technology (ICONSET), 2011 International Conference on, pp. 119-124, 2011.

[80] Wikipedia, "Wikipedia antiferromagnetism," Wikipedia, 2015. 
[81] K. K. Mallick, P. Shepherd, and R. J. Green, "Magnetic properties of cobalt substituted m-type barium hexaferrite prepared by co-precipitation," JMMM, 2006 .

[82] W. Kezhu, J. Renpei, and L. Shigen, "New technique of microwave ferrite and application," National Defense Industry Press, 2013.

[83] E. Schlömann, "Behavior of ferrites in the microwave frequency range," J. App. Phys, vol. 41, no. 1, p. 529, 1970.

[84] P. Gelin and K. B. Pichavant, "New consistent model for ferrite permeability tensor with arbitrary magnetization state," IEEE Trans. MTT, vol. 45, no. 8, pp. 1185-1192, 1997.

[85] G. T. Rado, "Theory of the microwave permeability tensor and faraday effect in nonsaturated ferromagnetic materials," Phys. Rev, vol. 89, p. 529, 1953.

[86] M. Igarashi and Y. Naito, "Tensor permeability of partially magnetized ferrites," IEEE Trans. Magnetics, vol. 13, no. 5, pp. 1664-1668, 1977.

[87] M. Igarashi and Y. Natio, "Parallel component $u_{z}$ of partially magnetized microwave ferrites," IEEE Trans. MTT, vol. 29, no. 6, pp. 568-571, 1981.

[88] P. Gelin and P. Quéffélec, "Generalized permeability tensor model: Application to barium hexaferrite in a remanent state for self-biased circulators," IEEE Trans. Magnetics, vol. 44, no. 1, pp. 24-31, 2008.

[89] P. Quéffélec, M. L. Floc'h, and P. Gelin, "New method for determining the permeability tensor of magnetized ferrites in a wide frequency range," IEEE Trans. MTT, vol. 48, no. 8, pp. 1344-1351, 2000.

[90] P. Gelin, P. Quéffélec, and F. L. Pennec, "Effect of domain and grain shapes on the dynamical behavior of polycrystalline ferrites: Application to the initial permeability," IEEE Trans. MTT, vol. 29, no. 6, pp. 568-571, 1981.

[91] J. J.Green and F. Sandy, "Microwave characterization of partially magnetized ferrites," IEEE Trans. MTT, vol. 22, no. 6, pp. 641-645, 1974.

[92] B. Lax and K.J.Button, "Microwave ferrites and ferrimagnetics," McGraw-Hill NewYork, 1962.

[93] E. ElectroScience, "Esl40012 ferrite ltcc tape," ESL ElectroScience, no. 6, 2015. 
[94] D. Vincent, T. Rouiller, C. Simovsky, B. Bayard, and G. Noyel, "A new broadband method for magnetic thin-film characterization in the microwave range," IEEE Trans. MTT, vol. 53, no. 4, pp. 1174-1180, 2005.

[95] P.L.Fulmek, P. Haumer, I. Atassi, B.Schweighofer, and H. Wegleiter, "Magnetic DC-properties of LTCC-ferrite material and their temperature dependence," IEEE Trans. Magnetics, vol. 48, no. 4, pp. 1541-1544, 2012.

[96] J. R. Bray, K. T. Kautio, and L. Roy, "Characterization of an experimental ferrite LTCC tape system for microwave and millimeter-wave applications," IEEE Trans. Advanced Packaging, vol. 27, no. 3, pp. 558-565, 2004.

[97] F. Ghaffar and A. Shamim, "A ferrite LTCC based dual purpose helical antenna providing bias for tunability," Antennas and Wireless Propagation Letters, IEEE, vol. 14, pp. 831-834, 2015.

[98] A. Abdul Ghaffar, F. ; Shamim, "A partially magnetized ferrite LTCC based siw phase shifter for phased array applications," IEEE Trans. Magnetics, to be published. Early Access.

[99] E. Arabi and A. Shamim, "The effect of self-heating on the performance of a tunable filter with embedded windings in a ferrite LTCC package," IEEE Transactions on Components, Packaging and Manufacturing Technology, vol. 5, no. 3, pp. 365-371, 2015.

[100] H. J. Carlin, "Principles of gyrator networks," Proc. Modern Advances in Microwave Tech. Symp, pp. 175-204, 1954.

[101] B. A. Auld, "The synthesis of symmetrical waveguide circulators," IRE Transactions on Microwave Theory and Techniques, vol. 7, no. 2, pp. 238-246, 1959.

[102] G. Buehler and A. Eikenberg, "Stripline Y-circulator for the 100 to $400 \mathrm{mc}$ region," Proc. IRE, vol. 49, p. 518, 1961.

[103] J. Clark, "Perturbation techniques for miniaturized coaxial Y- junction circulator," Journal of Applied Physics, vol. 32, p. 3238, 1961.

[104] L. Davis, U. Milano, and J. Saunders, "A stripline L-band compact circulator," Proc. IRE, vol. 48, p. 115, 1960.

[105] S. Yoshida, "Stripline Y-circulator," Proc. IRE, vol. 48, p. 1664, 1960. 
[106] M. Grace and F. Arams, "Three-port ring circulators," Journal of Applied Physics, vol. 48, p. 1497, 1960.

[107] J. Pippin, "Microwave ferrite device," Microwave Journal, vol. 11, pp. 29-45, 1960.

[108] J. S. Chang, "Master report: The design of three-port strip line circulator," University of Missouri Rolla-USA, 1969.

[109] D.D.Grieg and H.F.Englemann, "Microstrip-a new transmission technique for the klilomegacycle range," Proceedings of the IRE, vol. 40, no. 12, pp. 16441650,1952 .

[110] B. Hershenov, "X-band microstrip circulator," Proceedings of the IEEE, vol. 54, no. 12, pp. 2022-2023, 1966.

[111] R. Trambarulo, "A 30-GHz inverted-microstrip circulator (correspondence)," IEEE Trans. MTT, vol. 19, no. 7, pp. 662-664, 1971.

[112] B. Peng, W. Zhang, Y. Sun, J. Lin, W. Zhang, and H. Li, "Design of microstrip Y-junction circulator based on ferrite thin films," Microwave Conference Proceedings (CJMW), 2011 China-Japan Joint, pp. 1-4, 2011.

[113] W. Peng, Bin; Huizhong Xu; Hui Li; Wenxu Zhang; Yuan Wang; Zhang, "Self-biased microstrip junction circulator based on barium ferrite thin films for monolithic microwave integrated circuits," IEEE Trans. Magnetics, vol. 47, no. 6, pp. 1674-1677, 2011.

[114] C. P. Wen, "Coplanar waveguide, a surface strip transmission line suitable for nonreciprocal gyromagnetic device applications," Microwave Symposium, 1969 G-MTT International, pp. 110-115, 1969.

[115] B. Bayard, D. Yincent, C. R. Simovski, and G. Noyel, "Electromagnetic study of a ferrite coplanar isolator suitable for integration," IEEE Trans. MTT, vol. 51, no. 7, pp. 1809-1814, 2003.

[116] N. Ogasawara and M. Kaji, "Coplanar-guide and slot-guide junction circulators," Electronics Letters, vol. 7, no. 9, pp. 220-221, 1971.

[117] B. Wang, Y.and Peng and W.-L. Zhang, "Simulation of self-biased coplanar circulator using barium ferrite thin films," Microwave and Millimeter Wave Technology (ICMMT), 2010 International Conference on, pp. 543-546, 2010. 
[118] T. Boyajian, D. Vincent, S. Neveu, M. LeBerre, and J. Rousseau, "Coplanar circulator made from composite magnetic material," Microwave Symposium Digest (MTT), 2011 IEEE MTT-S International, 2011.

[119] J. B. Davies and P. Cohen, "Theoretical design of symmetrical junction stripline circulators," IEEE Trans. MTT, vol. 11, no. 6, pp. 506-512, 1963.

[120] R. E. Neidert and P. M. Phillips, "Losses in Y-junction stripline and microstrip ferrite circulators," IEEE Trans. MTT, vol. 41, no. 6, pp. 1081-1086, 1993.

[121] C. K. Seewald and J. R. Bray, "Ferrite-filled antisymmetrically biased rectangular waveguide isolator using magnetostatic surface wave modes," IEEE Trans. MTT, vol. 58, no. 6, pp. 1493-1501, 2010.

[122] S. Yang, D. Vincent, J. R. Bray, and L. Roy, "Ferrite ltcc edge-guided circulator," European Microwave Conference 2014, Rome, pp. 1540-1543, 2014.

[123] R. Soohoo, "Theory and application of ferrites," Englewood Cliffs, NJ:PrenticeHall, pp. 83-111, 1960.

[124] S. Yang, L. Roy, J. R. Bray, and D. Vincent, "Modeling and simulation of a partially-magnetized ferrite ltcc circulator," IEEE-MTT-Numerical Electromagnetic and Multiphysics Modeling and Optimization for RF, Microwave, and Terahertz Applications, 2015. 


\section{Publications}

\section{Journal}

Shicheng Yang; Vincent, D.; Bray, J.R.; Roy, L., "Study of a Ferrite LTCC Multifunctional Circulator With Integrated Winding," in Components, Packaging and Manufacturing Technology, IEEE Transactions on , vol.5, no.7, pp.879-886, July 2015, doi: 10.1109/TCPMT.2015.2440660

\section{International Conferences}

Shicheng Yang; Vincent, D.; Bray, J.R.; Roy, L., "Ferrite LTCC edge-guided circulator," in Microwave Conference (EuMC), 2014 44th European , vol., no., pp.1540-1543, 6-9 Oct. 2014, doi: 10.1109/EuMC.2014.6986743

Modeling and Simulation of a Partially-Magnetized Ferrite LTCC Circulator", IEEE Numerical Electromagnetic and Multiphysics Modeling and Optimization for RF, Microwave, and Terahertz Applications, August, Ottawa, Canada, 2015

\section{National Conference}

Shicheng Yang, Didier Vincent, Joey R Bray, Langis Roy, "Circulateur LTCC Ferrite large-bande à structures empilées en bande K", 19émes Journées Nationales Micro-ondes, 2015, Bordeaux, France, 2015 
Appendix A

S-parameters calculation 
APPENDIX A. S-PARAMETERS CALCULATION

171

Calculation of $S$-parameters (Stripling Circulator)

Before calculation:

$$
\begin{aligned}
& \mu_{0}=4 \pi \times 10^{-7} \mathrm{H} / \mathrm{m} \\
& \varepsilon_{0}=8.854 \times 10^{-12} \mathrm{~F} / \mathrm{m}
\end{aligned}
$$

$\varepsilon_{f}=14.6 \%$ relative permittivity of ferrite $\varepsilon_{d}=14.6 \%$ relative permittivity of dielectric The roots of $J_{n}^{\prime}(x)=0$ are:

$$
\left\{\begin{array}{l}
x_{11}=1.84118378134066 \\
x_{21}=3.054232692822714 \\
x_{31}=4.201 \\
x_{12}=5.33144277352503
\end{array}\right.
$$

$W_{C}=2 \pi f_{c} \%$ circulator's operating frequency.

Tensor of Polder:

$M_{s}=4000$ Gauss

$H_{i} \%$ internal $H_{i}$ field.

$\alpha=0.35 \%$ damping factor

$$
\begin{aligned}
& \gamma=2 \pi \times 28 \times 10^{g}=1.759 \times 10^{\prime \prime} \mathrm{c} / \mathrm{kg} \\
& \omega_{m}=\gamma_{0} \mu_{0} \mu_{s} \\
& \omega_{\gamma}=\mu_{0} H_{0} \gamma \\
& \mu=\frac{\left(\omega_{r}+j 2 \omega\right) \omega_{m}}{\left(\omega_{r}+j 2 \omega\right)^{2}-\omega^{2}}+1 \\
& k=\frac{w \omega_{m}}{\left(\omega_{r}+j 2 \omega\right)^{2}-\omega^{2}}
\end{aligned}
$$

PI. 
APPENDIX A. S-PARAMETERS CALCULATION

172

$$
[\mu]=\left[\begin{array}{ccc}
\mu & j k & 0 \\
-j k & \mu & 0 \\
0 & 0 & 1
\end{array}\right] \Leftrightarrow z \text { biased. }
$$

We can also calculate:

$$
\begin{aligned}
& \mu_{\text {eff }}=\frac{\mu^{2}-k^{2}}{\mu} \\
& k_{c}=w_{c} \sqrt{\varepsilon_{0} \mu_{0} \varepsilon_{f} \mu_{\text {eff }}} \\
& Z_{\text {eff }}=\sqrt{\frac{\mu_{0} \mu_{\text {eff }}}{\varepsilon_{0} \varepsilon_{f}}}
\end{aligned}
$$

$R=\frac{x_{11}}{k_{c}} \%$ the radius of ferrite/circulation (mode 1 , we can cello use $x_{21} x_{3}, \ldots$ for other modes).

$k_{d}=w_{c} \sqrt{\varepsilon_{0} \mu_{0} \varepsilon_{d}} \%$ progagation constant in dielectric.

$$
\begin{aligned}
& z_{d_{0}}=\frac{120 \pi}{\sqrt{\varepsilon_{d}}} \\
& \Psi_{c}=\frac{\pi}{\sqrt{3}(1.84)} \frac{z_{d}}{z_{\text {eff }}}\left|\frac{\mu}{k}\right| \% \text { coupling angle. }
\end{aligned}
$$

Calculation begins:

Green Foretion:

$$
G\left(r, \phi ; R, \phi^{\prime}\right)=-\frac{i Z_{e f f} J_{0}(k r)}{2 \pi J_{0}^{\prime}(x)}+\frac{Z_{\text {eff }}}{\pi} \sum_{n=1}^{\infty} \frac{\frac{k}{\mu} \frac{n J_{n}(x)}{x} \sin \left(\phi-\phi^{\prime}\right)-i J_{n}^{\prime}(x) \cos n\left(\phi-\phi^{\prime}\right)}{\left[J_{n}^{\prime}(x)\right]^{2}-\left[\frac{k}{\mu} \frac{n J_{n}(x)}{x}\right]^{2}} J_{n}(k r)
$$

Magnetic wall boundary condition is applied:

$$
H \phi= \begin{cases}H_{1} & -\frac{\pi}{3}-\psi<\phi<-\frac{\pi}{3}+\psi \\ H_{2} & \frac{\pi}{3}-\psi<\phi<\frac{\pi}{3}+\psi \\ H_{3} & \pi-\psi<\phi<\pi+\psi\end{cases}
$$

P. 2 
APPENDIX A. S-PARAMETERS CALCULATION

173

Then:

$$
E_{z}\left(R, \phi^{\prime}\right)=\int_{c} G\left(\phi \mid \phi^{\prime}\right) H \phi\left(R, \phi^{\prime}\right) d \phi
$$

$\Rightarrow$ Impedance at each port:

$$
\eta\left(\phi \mid \phi_{0}\right)=\frac{E_{z}\left(R, \phi_{0}\right)}{H_{\phi}\left(R, \phi_{0}\right)}=\int_{c} G\left(\phi \mid \phi_{0}\right) d \phi
$$

$\Rightarrow$ The observation port is assumed to be $\phi_{0}=-\frac{\pi}{3}$

$S_{0} \eta_{11}=\int_{c} G\left(\phi \mid-\frac{\pi}{3}\right) d \phi \quad-\frac{\pi}{3}-\psi<\phi<-\frac{\pi}{3}+\psi$

$$
\longrightarrow \eta_{11}=\int_{-\frac{\pi}{3} *-\psi}^{-\frac{\pi}{3}+\psi} G\left(\phi \mid-\frac{\pi}{3}\right) d \phi
$$

Assume that:

$$
\begin{aligned}
& A=\frac{Z_{\text {eff }}}{\pi} \frac{\frac{k}{\mu} \frac{n J_{n}(x)}{x}}{\left[J_{n}^{\prime}(x)\right]^{2}-\left[\frac{k}{\mu} \frac{n J_{n}(x)}{x}\right]^{2}} J_{n}(k r) \\
& B=\frac{z_{\text {eff }}}{\pi} \frac{i J_{n}^{\prime}(x)}{\left[J_{n}^{\prime}(x)\right]^{2}-\left[\frac{k}{\mu} \frac{n J_{n}(x)}{x}\right]^{2}} J_{n}(k r) \\
& a_{0}=-\frac{i Z_{\text {eff }} J_{0}(k r)}{2 \pi J_{0}^{\prime}(x)}
\end{aligned}
$$

PB. 
APPENDIX A. S-PARAMETERS CALCULATION

174

$$
\begin{aligned}
& \eta_{11}=\int_{-\frac{\pi}{3}-\psi}^{-\frac{\pi}{3}+\psi} a_{0} d \phi+\int_{-\frac{\pi}{3}-\psi}^{-\frac{\pi}{3}+\psi} A \sin n\left(\phi+\frac{\pi}{3}\right)-B \cos n\left(\phi-\frac{\pi}{3}\right) d \phi \\
& =2 \psi_{\cos } *-\left.\frac{A}{n} \cos n\left(\phi+\frac{\pi}{3}\right)\right|_{-\frac{\pi}{3}-\psi} ^{-\frac{\pi}{3}+\psi}-\left.\frac{B}{n} \sin n\left(\phi+\frac{\pi}{3}\right)\right|_{-\frac{\pi}{3}-\psi} ^{-\frac{\pi}{3}+\psi} \\
& =2 \psi a_{0}-\frac{A}{n}[\cos n \psi-\cos (-n \psi)]-\frac{B}{n}[\sin n(\psi)-\sin (-n \psi)] \\
& =2 \psi a_{0}-2 B \psi \sin c(n \psi) \\
& \left.\eta_{21}=\int_{\frac{\pi}{3}-\psi}^{\frac{\pi}{3}+\psi} G(\phi)-\frac{\pi}{3}\right) d \phi \\
& =\int_{\frac{\pi}{3}-\psi}^{\frac{\pi}{3}+\psi} a_{0} d \phi+\int_{-\frac{\pi}{3}-\psi}^{\frac{\pi}{3}+\psi} A \sin n\left(\phi+\frac{\pi}{3}\right)-B \cos n\left(\phi+\frac{\pi}{3}\right) d \phi \\
& =2 \psi a_{0}-\left.\frac{A}{n} \cos n\left(\phi+\frac{\pi}{3}\right)\right|_{\frac{\pi}{3}-\psi} ^{\frac{\pi}{3}+\psi}-\left.\frac{B}{n} \sin n\left(\phi+\frac{\pi}{3}\right)\right|_{\frac{\pi}{3}-\psi} ^{\frac{\pi}{3}+\psi} \\
& =2 \psi a_{0}-\frac{A}{n}\left[\cos n\left(\frac{2 \pi}{3}+\psi\right)-\cos \pi\left(\frac{2 \pi}{3}-\psi\right)\right]-\frac{B}{n}\left[\sin \left(\frac{2 \pi}{3}+\psi\right)-\sin \left(\frac{2 \pi}{3}-\psi\right)\right] \\
& =2 \psi c_{10}-\frac{A}{n}\left(-2 \sin \frac{2 \pi \pi}{3} \sin \psi\right)-\frac{B}{n}\left(2 \cos \frac{2 n \pi}{3} \sin \psi \psi\right) \\
& =2 \psi a_{0}+2 \psi\left[A \sin \frac{2 n \pi}{3} \sin c(n \psi)-B \cos \frac{2 n \pi}{3} \sin c(n \psi)\right] \\
& =2 \psi a_{0}+2 \psi \sin c(n \psi)\left[A \sin \frac{2 n \pi}{3}-\beta \cos \frac{2 n \pi}{3}\right] \\
& \eta_{3)}=\int_{\pi-\psi}^{\pi+\psi} G\left(\phi \mid-\frac{\pi}{3}\right) d \phi \\
& =\int_{\pi-\psi}^{\pi+\psi} a_{0} d \phi+\int_{\pi-\psi}^{\pi+\psi} A \sin m\left(\phi+\frac{\pi}{3}\right)-B \cos n\left(\phi+\frac{\pi}{3}\right) d \phi \\
& =2 \psi a_{0}-\left.\frac{A}{n} \cos n\left(\phi+\frac{\pi}{3}\right)\right|_{\pi-\psi} ^{\pi+\psi}-\left.\frac{B}{n} \sin n\left(\phi+\frac{\pi}{3}\right)\right|_{\pi-\psi} ^{\pi+\psi} \\
& =2 \psi a_{0}-\frac{A}{n}\left[\cos n\left(\frac{4 \pi}{3}+\psi\right)-\cos n\left(\frac{4 \pi}{3}-\psi\right)\right]-\frac{B}{n}\left[\sin n\left(\frac{4 \pi}{3}+\psi\right)-\sin n\left(\frac{4 \pi}{3}-\psi\right)\right] \\
& =2 \psi a_{0}+2 \psi \sin c(n \psi)\left[A \sin \frac{4 n \pi}{3}-B \cos \frac{4 n \pi}{3}\right]
\end{aligned}
$$

P.4 
The final results of $[\eta]$ is:

$$
\begin{aligned}
& \eta_{11}=-\frac{i Z_{\text {eff }} J_{0}(k r)}{2 \pi J_{0}^{\prime}(x)} 2 \psi+\frac{Z_{\text {eff }}}{\pi} \sum_{n=1}^{\infty} \frac{-i 2 J_{n}^{\prime}(x) \psi \sin (n \psi)}{\left[J_{n}^{\prime}(x)\right]^{2}-\left[\frac{k}{\mu} \frac{n J_{n}(x)}{x}\right]^{2}} J_{n}(k r) \\
& \eta_{21}=-\frac{i z_{\text {eff }} J_{0}(k r)}{2 \pi J_{0}^{\prime}(x)} 2 \psi+\cdots \\
& +\frac{Z_{\text {eff }}}{\pi} \sum_{n=1}^{\infty} \frac{2 \psi \sin ((n))\left[\frac{k}{\mu} \frac{n J_{n}(x)}{4} \sin \frac{2 n \pi}{3}-i J_{n}^{\prime}(x) \cos \frac{2 n \pi}{3}\right]}{\left[J_{n}^{\prime}(x)\right]^{2}-\left[\frac{k}{\mu} \frac{n J_{n}(x)}{x}\right]^{2}} J_{n}(k r) \text {. } \\
& \eta_{B_{1}}=-\frac{i z_{\text {eff }} J_{0}(k r)}{2 \pi J_{0}^{\prime}(x)} 2 \psi+\cdots \\
& {[\eta]=\left[\begin{array}{lll}
\eta_{11} & \eta_{31} & \eta_{21} \\
\eta_{21} & \eta_{11} & \eta_{31} \\
\eta_{31} & \eta_{21} & \eta_{11}
\end{array}\right] \stackrel{\text { Nomalized by }}{\underset{z_{0}}{\mathrm{y}}=\frac{120 \pi}{\sqrt{\varepsilon_{0}}}}[z]=\left[\begin{array}{lll}
z_{11} & z_{31} & z_{21} \\
z_{21} & z_{11} & z_{31} \\
z_{31} & z_{21} & z_{11}
\end{array}\right]} \\
& \leftrightarrow \text { Now we calculate the }[s] \text { by }[s]=[z+I]^{-1}[z+I] \\
& \begin{array}{l}
{[z+1]=\left[\begin{array}{lll}
z_{11}+1 & z_{31} & z_{21} \\
z_{21} & z_{11}+1 & z_{31} \\
z_{31} & z_{21} & z_{11}+1
\end{array}\right]} \\
{[z+1]^{-1}=\frac{1}{\operatorname{det}(z+1)} \operatorname{adj}(z+1)}
\end{array} \\
& \operatorname{adj}(z+1)=\left[\begin{array}{ccc}
\left(z_{11}+1\right)^{2}-z_{21} z_{31} & z_{21}^{2}-z_{31}\left(z_{11}+1\right) & z_{31}^{2}-z_{21}\left(z_{11}+1\right) \\
z_{3}^{2}-z_{21}\left(z_{11}+1\right) & \left(z_{11}+1\right)^{2}-z_{21} z_{31} & z_{21}^{2}-z_{31}\left(z_{11}+1\right) \\
z_{21}^{2}-z_{31}\left(z_{11}+1\right) & z_{31}^{2}-z_{21}\left(z_{11}+1\right) & \left(z_{11}+1\right)^{2}-z_{21} z_{31}
\end{array}\right] \\
& \text { if } A=\left(z_{11}+1\right)^{2}-z_{11} z_{31} \\
& B=z_{31}^{2}-z_{21}\left(z_{11}+1\right) \\
& C=z_{21}^{2}-z_{31}\left(z_{11}+1\right) \text {. }
\end{aligned}
$$


APPENDIX A. S-PARAMETERS CALCULATION

176

$$
\begin{aligned}
& {[Z+I]^{*}=\left[\begin{array}{lll}
A & C & B \\
B & A & C \\
C & B & A
\end{array}\right]} \\
& \operatorname{det}|z+I|=\left(z_{11}+1\right)^{3}+z_{21}^{3}+z_{31}^{3}-3\left(z_{11}+1\right) z_{21} z_{31} \\
& {[s]=[z+1]^{-1}[z-I]} \\
& =\frac{1}{\operatorname{det}|z+I|}\left[\begin{array}{ccc}
A & C & B \\
B & A & C \\
C & B & A
\end{array}\right]\left[\begin{array}{ccc}
z_{11}-1 & z_{31} & z_{21} \\
z_{21} & z_{11}-1 & z_{31} \\
z_{31} & z_{21} & z_{11}-1
\end{array}\right] \\
& =\frac{1}{\operatorname{det}|z+1|}\left[\begin{array}{lll}
A\left(z_{11}-1\right)+C z_{21}+B z_{31} & A z_{31}+C\left(z_{11}-1\right)+B z_{21} & A z_{21}+C z_{31}+B\left(z_{11}-1\right) \\
B\left(z_{11}-1\right)+A z_{21}+C z_{31} & B z_{31}+A\left(z_{11}-1\right)+C z_{31} & B z_{21}+A z_{31}+C\left(z_{11}-1\right) \\
C\left(z_{11}-1\right)+B z_{21}+A z_{31} & C z_{31}+B\left(z_{11}-1\right)+A z_{21} & C z_{21}+B z_{31}+A\left(z_{11}-1\right)
\end{array}\right] \\
& \Rightarrow \\
& S_{11}=\frac{1}{\operatorname{det}|z+I|}\left[A\left(z_{11}-1\right)+\left(z_{21}+B z_{31}\right]\right. \\
& =\frac{1}{\operatorname{det}|z+1|}\left\{\left[\left(z_{11}+1\right)^{2}-z_{21} z_{31}\right]\left(z_{11}-1\right)+z_{21}^{3}-z_{21} z_{31}\left(z_{11}+1\right)+z_{31}^{3}-z_{31} z_{21}\left(z_{11}+1\right)\right\}
\end{aligned}
$$

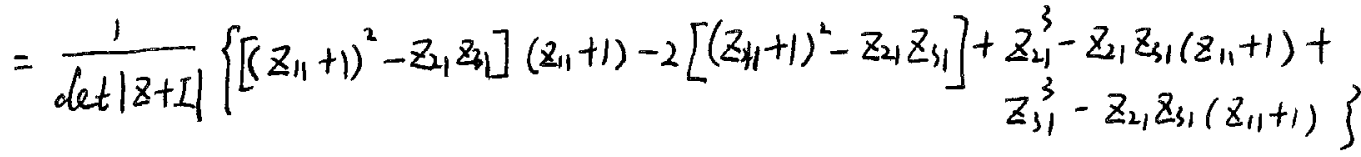

$$
\begin{aligned}
& =1+\frac{-2}{\operatorname{det}|z+I|}\left[\left(z_{11}+1\right)^{2}-z_{2,} z_{31}\right] \\
& =1+\frac{-2\left[\left(z_{11}+1\right)^{2}-z_{21} z_{3}\right] z_{d}}{\left(z_{11}+1\right)^{3}+z_{21}^{3}+z_{31}^{3}-3 z_{21} z_{31}\left(z_{11}+1\right)}
\end{aligned}
$$

P. 6 
APPENDIX A. S-PARAMETERS CALCULATION

177

$$
\begin{aligned}
& S_{21}=\frac{1}{\operatorname{det}|z+I|}\left[B\left(z_{11}-1\right)+A z_{21}+C z_{31}\right] \\
& \begin{array}{r}
=\frac{1}{\operatorname{det}|z+I|}\left\{\left[z_{31}^{2}-z_{21}\left(z_{11}+1\right)\right]\left(z_{11}+1\right)-2\left[z_{31}^{2}-z_{21}\left(z_{11}+1\right)\right]+\left[\left(z_{11}+1\right)^{2}-z_{21} z_{31}\right] z_{21}\right. \\
\left.+\left[z_{21}^{2}-z_{31}\left(z_{11}+1\right)\right] z_{31}\right\}
\end{array} \\
& =\frac{1}{\operatorname{det}|z+1|}\left\{-2\left[z_{31}^{2}-z_{21}\left(z_{11}+1\right)\right]\right\} \\
& =-\frac{2\left[z_{31}^{2}-z_{21}\left(z_{11}+1\right)\right] z_{d}}{\left(z_{11}+1\right)^{3}+z_{21}^{3}+z_{31}^{3}-3 z_{21} z_{31}\left(z_{11}+1\right)} \\
& S_{31}=\frac{1}{\operatorname{det}|z+I|}\left[c\left(Z_{11}-1\right)+B Z_{21}+A Z_{31}\right] \\
& =\frac{1}{\operatorname{det}|z+I|}\left\{\left[z_{21}^{2}-z_{31}\left(z_{11}+1\right)\right]\left(z_{11}+1\right)-2\left[z_{21}^{2}-z_{31}\left(z_{11}+1\right)\right]+z_{31}^{2} z_{21}-z_{21}^{2}\left(z_{11}+1\right)\right. \\
& =-\frac{2\left[z_{21}^{2}-z_{31}\left(z_{11}+1\right)\right] z_{d}}{\left(z_{11}+1\right)^{3}+z_{21}^{3}+z_{31}^{3}-3 z_{21} z_{31}\left(z_{11}+1\right)} \\
& \left.+z_{31}\left(z_{11}+1\right)^{2}-z_{21} z_{31}^{2}\right] \\
& \Rightarrow S=\left[\begin{array}{lll}
s_{11} & s_{31} & s_{21} \\
s_{21} & s_{11} & s_{31} \\
S_{31} & s_{21} & s_{11}
\end{array}\right]
\end{aligned}
$$

Let's:

$$
\begin{array}{ll}
\text { Let's : } & \Rightarrow z_{11}+1=\frac{z_{11}+1}{2 z_{\text {eff }}} \quad \begin{array}{l}
j \pi c_{\text {eff }} \\
j \pi
\end{array} \\
c_{2}=j \pi \frac{z_{31}}{2 z_{\text {eff }}} & \Rightarrow z_{31}=\frac{2 c_{2} z_{\text {eff }}}{j \pi} . \\
c_{3}=j \pi \frac{z_{21}}{2 z_{\text {eff }}} & \Rightarrow z_{21}=\frac{2 c_{3} z_{\text {eff }}}{j \pi}
\end{array}
$$

P. 7 . 
APPENDIX A. S-PARAMETERS CALCULATION

178

$$
\begin{aligned}
& S_{11}=1+\frac{-2\left[\left(z_{11}+1\right)^{2}-z_{21} z_{31}\right] z_{d}}{\left(z_{11}+1\right)^{3}+z_{21}^{3}+z_{31}^{3}-3 z_{21} z_{31}\left(z_{11}+1\right)} \\
& =1+(-2) \frac{1}{\frac{2 z_{f}}{j \pi z_{d}}} \cdot \frac{c_{1}^{2}-c_{2} c_{3}}{c_{1}^{3}+c_{2}^{3}+c_{3}^{3}-3 c_{1} c_{2} c_{3}} \\
& =1+\frac{\pi Z_{d}}{j z_{\text {eff }}} \frac{c_{1}^{2}-c_{2} c_{3}}{c_{1}^{3}+c_{2}^{3}+c_{3}^{3}-3 c_{1} c_{2} c_{3}} \\
& S_{21}=\frac{-2\left[z_{31}^{2}-z_{21}\left(z_{11}+1\right)\right] z_{2}}{\left(z_{11}+1\right)^{3}+z_{21}^{3}+z_{31}^{3}-3 z_{21} z_{31}\left(z_{11}+1\right)} \\
& =-2 \times \frac{j \pi z_{d}}{2 z_{\text {eff }}} \cdot \frac{c_{2}^{2}-c_{1} c_{3}}{c_{1}^{3}+c_{2}^{3}+c_{3}^{3}-3 c_{1} c_{2} c_{3}} \\
& =\frac{\pi z_{d}}{j z_{\text {eff }}} \frac{c_{2}^{2}-c_{1} c_{3}}{c_{1}^{3}+c_{2}^{3}+c_{3}^{3}-3 c_{1} c_{2} c_{3}} \\
& S_{31}=\frac{\pi z_{d}}{j z_{\text {eff }}} \frac{c_{3}^{2}-c_{1} c_{2}}{c_{1}^{3}+c_{2}^{3}+c_{3}^{3}-3 c_{1} c_{2} c_{3}} \\
& {[s]=\left[\begin{array}{lll}
s_{11} & s_{31} & s_{21} \\
S_{21} & S_{11} & S_{31} \\
s_{31} & S_{21} & S_{11}
\end{array}\right]}
\end{aligned}
$$

Ps. 